228

\title{
Development of a Method for Predicting the Performance and Wear of PDC Drill Bits
}

David A. Glowka

Prepared by

Sandia National Laboratories

Albuquerque, New Mexico 87185 and Livermore, California 94550

for the United States Department of Energy 


\section{DISCLAIMER}

This report was prepared as an account of work sponsored by an agency of the United States Government. Neither the United States Government nor any agency Thereof, nor any of their employees, makes any warranty, express or implied, or assumes any legal liability or responsibility for the accuracy, completeness, or usefulness of any information, apparatus, product, or process disclosed, or represents that its use would not infringe privately owned rights. Reference herein to any specific commercial product, process, or service by trade name, trademark, manufacturer, or otherwise does not necessarily constitute or imply its endorsement, recommendation, or favoring by the United States Government or any agency thereof. The views and opinions of authors expressed herein do not necessarily state or reflect those of the United States Government or any agency thereof. 


\section{DISCLAIMER}

Portions of this document may be illegible in electronic image products. Images are produced from the best available original document. 


\begin{abstract}
Issued by Sandia National Laboratories, operated for the United States Department of Energy by Sandia Corporation.

NOTICE: This report was prepared as an account of work sponsored by an agency of the United States Government. Neither the United States Government nor any agency thereof, nor any of their employees, nor any of thei contractors, subcontractors, or their employees, makes any warranty, express or implied, or assumes any legal liability or responsibility for the accuracy, completeness, or usefulness of any information, apparatus, product, or pro ces disclosed, or represents that its use would mot infringe privately owned cess disclosed, or represents that its use would not infringe privately owned rights. Reference herein to any specific commercial product, process, or service by trade name, trademark, manufacturer, or otherwise, does not necessarily constitute or imply its endorsement, recommendation, or favoring by the United States Government, any agency thereof or any of thei contractors or subcontractors. The views and opinions expressed herein do not necessarily state or reflect those of the United States Government, any agency thereof or any of their contractors or subcontractors.
\end{abstract}

Printed in the United States of America Available from

National Technical Information Service

U.S. Department of Commerce

5285 Port Royal Road

Springfield, VA 22161

NTIS price codes

Printed copy: A10

Microfiche copy: A01 


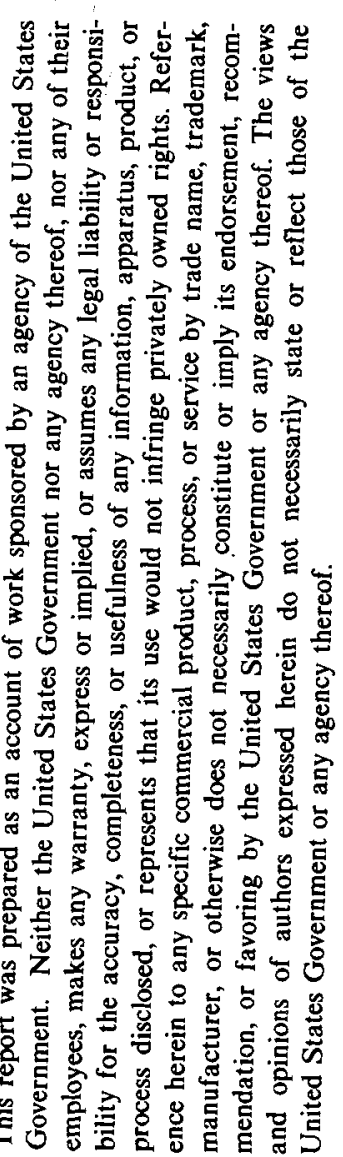

SAND $-86-1745$

DE88 001450

Distribution Category UC-66c

SAND86-1745

Unlimited Distribution

Printed June 1987

DEVELOPMENT OF A METHOD FOR PREDICTING

THE PERFORMANCE AND WEAR OF PDC DRILL BITS

David A. Glowka

Geotechnical Design Division 6314

Sandia National Laboratories

Albuquerque, New Mexico 87185

A method is developed for predicting cutter forces, temperatures, and wear on PDC bits as well as integrated bit performance parameters such as weight-on-bit, drilling torque, and bit imbalance. A computer code called PDCWEAR has been developed to make this method available as a tool for general bit design and analysis $f$. The method uses single-cutter data to provide a measure of rock drillability and employs theoretical considerations to account for interaction among closely spaced cutters on the bit. Experimental data are presented to establish the effects of cutter size and wearflat area on the forces that develop during rock cutting. Waterjet assistance is shown to significantly reduce cutting forces, thereby potentially extending bit life and reducing weight-on-bit and torque requirements in hard rock. The effects of several other design and operating parameters on bit life and drilling performance are also investigated.

$\dagger$ This code and sample problem input data are available from:

National Energy Software Center

Argonne National Laboratory

9700 South Cass Avenue

Argonne, IL 60439

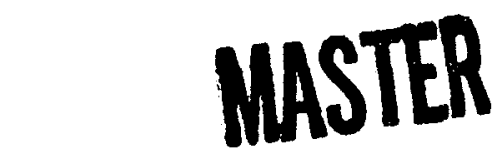


TABLE OF CONTENTS

Page

1.0 Introduction $\quad 1$

2.0 Development of a PDC Cutting Force Model 7

2.1 Experimental Set-up, Procedures, and Conditions 10

2.2 Test Results: Dry, Non-Interacting Cuts 18

2.3 Test Results: Non-Interacting Cuts with Waterjet
Assistance

2.4 Test Results: Interacting Cuts 29

2.5 Analysis of Cutter Interaction Effects 29

2.6 Other Observations 33

3.0 Development of the Computer Code, PDCWEAR 39

$\begin{array}{llr}3.1 & \text { Cutter Interaction Theory } & 39\end{array}$

3.2 Forces and Moments Acting on the Bit 48

3.3 Cutter Wearflat Temperatures $r$

3.4 Cutter Wear Rates $\quad 53$

4.0 Demonstration of the Use of PDCWEAR 59

4.1 Input Parameters $\quad 62$

4.2 Running the Program $\quad 65$

4.3 Tabulated Program Output 68

4.4 Discussion of Baseline Analysis Results 69

4.5 Other Program Features $\quad 80$

5.0 Predicted Effects of Design and Operation on Bit Performance 81

5.1 Effects of Bit Profile $\quad 81$

5.2 Effects of Cutter Placement Density 81

5.3 Effects of Bit Rotary Speed 88

5.4 Effects of Waterjet Assistance $\quad 88$

$\begin{array}{ll}5.5 \text { Effects of Wear Mode } & 91\end{array}$

$\begin{array}{lll}5.6 & \text { Discussion } & 93\end{array}$

$\begin{array}{lll}6.0 & \text { Conclusions } & 95\end{array}$

$\begin{array}{lll}7.0 & \text { Nomenclature } & 97\end{array}$

$\begin{array}{lll}8.0 & \text { Acknowledgment } & 101\end{array}$

$\begin{array}{llr}9.0 & \text { References } & 103\end{array}$

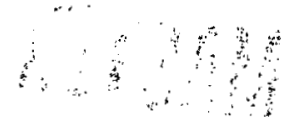




\section{Page}

$\overline{1}$

$\begin{array}{ll}\text { Appendix A - Experimental Single-Cutter Data } & 109\end{array}$

Appendix B - Listing of Fortram Program PDCWEAR 125

Appendix C - Thermal Numerical Modeling of a 0.75-Inch PDC Cutter 149

Appendix D - PDCWEAR Input Guide 153

Appendix E - Listing of Fortran Program FORMAT $\quad 165$

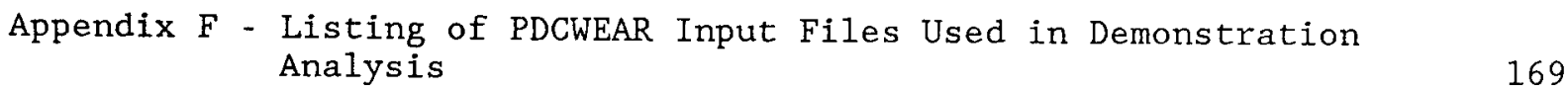

Appendix G - Partial PDCWEAR Output Listing for Demonstration Analysis 173

$\begin{array}{ll}\text { DISTRIBUTION LIST } & 189\end{array}$

List of Tables iv

List of Figures $\quad \mathrm{v}$ 


\section{LIST OF TABLES}

Table No.

Title

Page

I

II

III

A- 1

A -2

A -3

A -4

A -5

A- 6

A- 7

C-1

D-1

F-1

F- 2

F-3
Coefficients in Equations 38 and 39

55

Cutter Positions in Demonstration Analysis

60

Bit Operating Conditions in Demonstration Analysis

Description of PDC Cutters Used in

Laboratory Tests

110

Dry, Non-Interacting Cut Test Data for

Berea Sandstone

111

Dry, Non-Interacting Cut Test Data for

Sierra White Granite

116

Dry, Non-Interacting Cut Test Data for

Tennessee Marble

Waterjet-Assisted, Non-Interacting Cut Test

Data for Sierra White Granite

Dry, Interacting Cut Test Data for Berea

Sands tone

122

Dry, Interacting Cut Test Data for Sierra

White Granite

Computed Thermal Response Function for 0.75-Inch and 0.5-Inch Cutters

162

170

PDCWEAR Input File Containing Bit Design Data for Demonstration Analysis (BITDES.DAT)

171 Configuration Data for Demonstration Analysis (WEARCF.DAT)

172 
Figure 1 - Collection of 8-1/2 inch PDC bits, showing variations in bit shape and location and number of cutters.

Figure 2 - Single PDC cutter, showing front and side view.

Figure 3 - Schematic of a single PDC cutter mounted on the leading face of a bit and experiencing interaction with nearby cutters.

Figure 4 - Cutting edge profiles for sharp cutters on the leading face of a PDC bit.

Figure 5 - Milling machine used for linear single-cutter tests. 12

Figure 6 - Geometry of single-cutter tests with waterjet assistance. 13

Figure 7 - Vertical milling machine used for wearing PDC cutters in the laboratory.

Figure 8 - Cutting pattern used in non-interacting single-cutter tests.

Figure 9 - Cutting patterns used to simulate cutter interaction in single-cutter tests.

Figure 10 - Measured penetrating stresses with various wearflat configurations in dry, non-interacting cuts.

Figure 11 - Measured penetrating stresses with various wearflat configurations in dry, non-interacting cuts.

Figure 12 - Measured penetrating forces with sharp cutters in dry, non-interacting cuts.

Figure 13 - Measured drag coefficients with various wearflat configurations in dry, non-interacting cuts.

Figure 14 - Measured drag coefficients with various wearflat configurations in dry, non-interacting cuts.

Figure 15 - Measured drag coefficients with sharp cutters in dry, non-interacting cuts.

Figure 16 - Measured penetrating stresses in non-interacting cuts made with waterjet assistance.

Figure 17 - Measured drag coefficients in non-interacting cuts made with waterjet assistance.

Figure 18 - Measured penetrating stresses in dry, interacting cuts. 
Page

Figure 19 - Interacting cut patterns with large and small lateral spacing between cuts.

Figure 20 - Typical cutting pattern for a worn cutter on the leading face of a PDC bit.

Figure 21 - Computed effective depth of cut as a function of lateral distance to adjacent cuts in interacting cut tests.

Figure 22 - Predicted and measured penetrating stresses for dry, interacting cut tests.

Figure 23 - Circumferential wear angle found to develop on field worn cutters.

Figure 24 - Cut and cutter profiles for a general PDC cutter mounted on a bit.

Figure 25 - Schematic of the four coordinate systems used to describe cut and cutter profiles.

Figure 26 - Detailed definition of several points used in cutter geometry analysis.

Figure 27 - Schematic of algorithm used in PDCWEAR to compute $z$-coordinates of cutting profiles at each value of $x$.

Figure 28 - Schematic showing how cutter forces are integrated to produce bit performance parameters.

Figure 29 - Finite element mesh used to compute thermal response of 0.75 -inch cutters.

Figure 30 - Schematic of 8-1/2 inch bit design used in the demonstration analysis.

Figure 31 - Definition of several bit design parameters required as input for PDCWEAR.

Figure 32 - Predicted wearflat growth of cutter with highest wear rate in the demonstration analysis.

Figure 33 - Predicted wear distribution across bit in the demonstration analysis at designated stages of bit wear.

Figure 34 - Predicted cutter penetrating forces in the demonstration analysis.

Figure 35 - Predicted cutter wear ratios in the demonstration analysis. 
Figure 36 - Predicted cutter wearflat temperatures in the demonstration analysis.

Figure 37 - Predicted WOB at specified penetration rates in the demonstration analysis.

Figure 38 - Predicted drilling torque at specified penetration rates in the demonstration analysis.

Figure 39 - Predicted resultant bit side force at specified penetration rates in the demonstration analysis.

Figure 40 - Predicted resultant bit bending moment at specified penetration rates in the demonstration analysis.

Figure 41 - Schematic of bullet-nose bit design used in modified analysis to illustrate the effects of bit profile.

Figure 42 - Predicted cutter wear distribution across bit, showing the effects of bit profile.

Figure 43 - Specified penetration rate plotted as a function of predicted WOB to illustrate the effects of bit profile.

Figure 44 - Schematic of bit design used in modified analysis to illustrate the effects of cutter placement density.

Figure 45 - Predicted cutter wear distribution across bit, showing the effects of cutter placement density.

Figure 46 - Predicted cutter wear distribution across bit, showing the effects of rotary speed.

Figure 47 - Predicted cutter wear distribution across bit, showing the effects of waterjet assistance for selected cutters $(13-16)$.

Figure 48 - Predicted cutter wear distribution across bit, showing the effects of wear mode.

Figure D-1 - Measured heat transfer coefficients for various locations on a stud-mounted 0.5 -inch diameter compact cutter due to a uniform flow of water past the cutter.

Figure D-2 - Measured heat transfer coefficients for various locations on a stud-mounted 0.5 -inch diameter compact cutter due to impingement of a low-pressure ( 80 psi) waterjet.

Figure D-3 - Mean PDC cutter heat transfer coefficients under typical air and water drilling conditions. 


\subsection{INTRODUCTION}

Research has been conducted for several years at Sandia National Laboratories to foster the development of PDC bits for geothermal drilling. This work, sponsored by the Geothermal Technologies Division of the U. S. Department of Energy, has been directed toward the high-temperature, hardrock drilling environment that is typically found near geothermal resources. We have strived, however, to interpret the results to make them applicable to drilling environments of interest to the petroleum industry as well.

In high-temperature, hard-rock drilling environments, roller bits suffer extremely short lives and are a significant contributing factor to the high costs associated with geothermal drilling [1-4]. The primary cause of this short bit life is rapid wear of the bearings that support the roller cones. Loose bearings help accelerate gage wear on the bits, thereby leading to undergage holes. High downhole temperatures play a major role in accelerating roller bit wear because of: (1) temperature limitations on the effectiveness of elastomeric seals that might be used to seal the bearings from the abrasive drilling fluid; and (2) the adverse effects of frictional temperatures on the wear of rubbing surfaces.

A potential alternative to roller bits in many drilling applications is the type of bit known as the polycrystalline diamond compact (PDC) bit. This type of bit consists of a solid bit head onto which are attached multiple PDC cutters. A photograph of several PDC bit designs is shown in Figure 1. Note that the configuration of a PDC bit can vary in shape, size, number and placement of cutters, and hydraulic design. These and other variables determine the performance of a bit in a given application.

Shown in Figure 2 is a single stud-mounted PDC cutter. The thin black layer of material on the leading face of the cutter is the artificialdiamond compact, which provides the wear resistance that makes this type of cutter effective in drilling rock. The PDC layer is bonded to a tungsten carbide-cobalt (WC-Co) compact, which in turn is mounted to a WC-Co stud. The entire cutter assembly is then pressed into holes machined in the head of the bit. Some PDC bits are also made with matrix-mounted cutters. In this case, the WC-Co compacts with the PDC layers are brazed directly onto flat surfaces cast into the head of the bit.

The mechanical simplicity of PDC bits, particularly the associated advantage of no moving parts, led to Sandia's interest in this technology for geothermal drilling. Our earliest efforts concentrated on developing technology for effectively mounting PDC cutters to the bit body. Developments included: (1) a design for a more fracture-resistant cutter stud [5-7]; (2) test results that helped establish optimal cutter rake angles [8]; and (3) a diffusion bonding technique for attaching the WC-Co compact with its PDC layer to the stud for high-temperature applications [811].

Field and laboratory drilling tests in geothermal and other hard-rock formations helped identify the potential limitations of PDC bits under severe conditions $[5,6,10,12-20]$. Although PDC bits were found capable of drilling most formations as fast or faster that roller bits, bit life was found to be extremely formation-dependent. Field tests in the soft shales and sandstones of geothermal wells in the Imperial Valley demonstrated PDC 


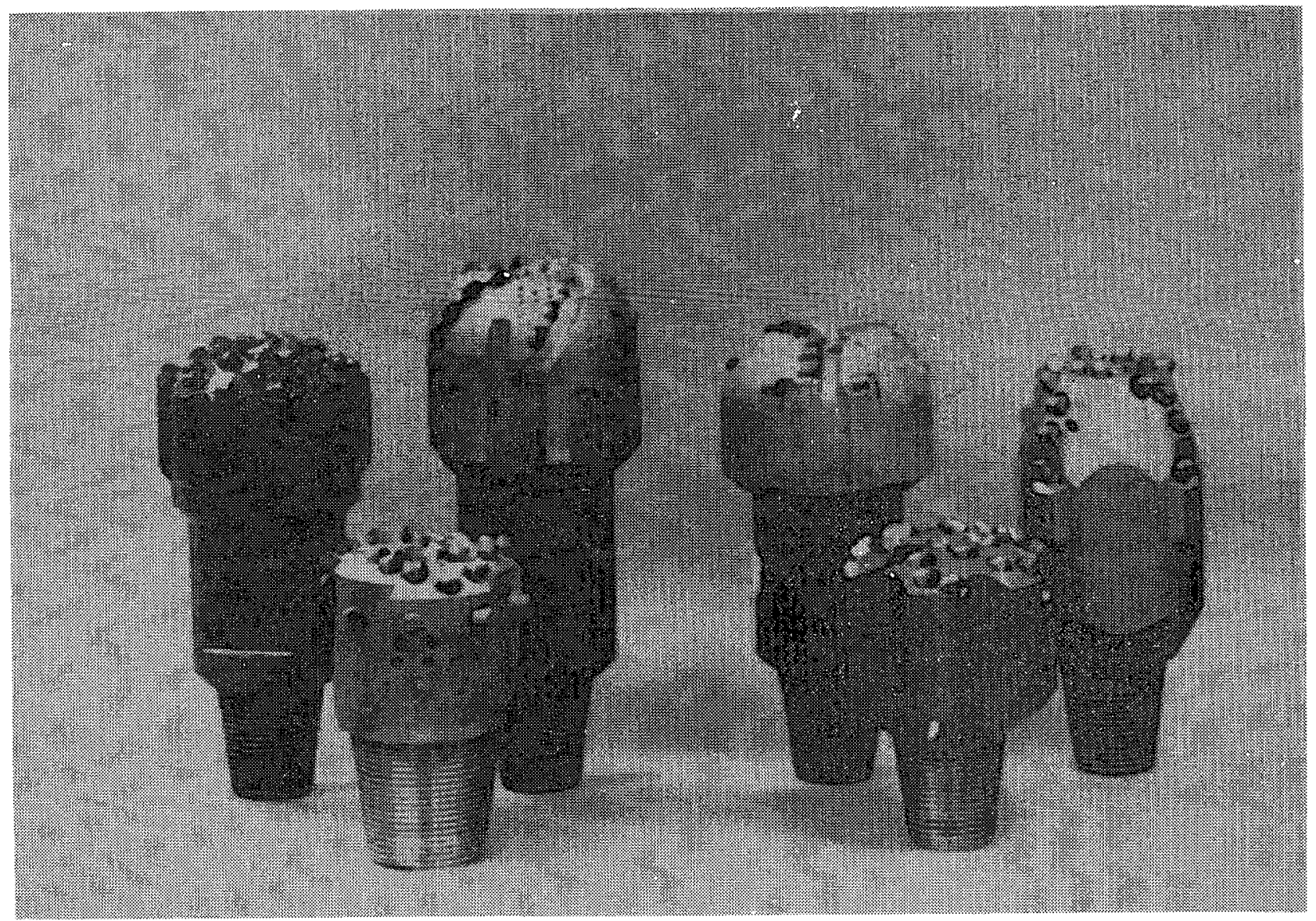

Figure 1 - Collection of 8-1/2 inch PDC bits, showing variations in bit shape and location and number of cutters. 


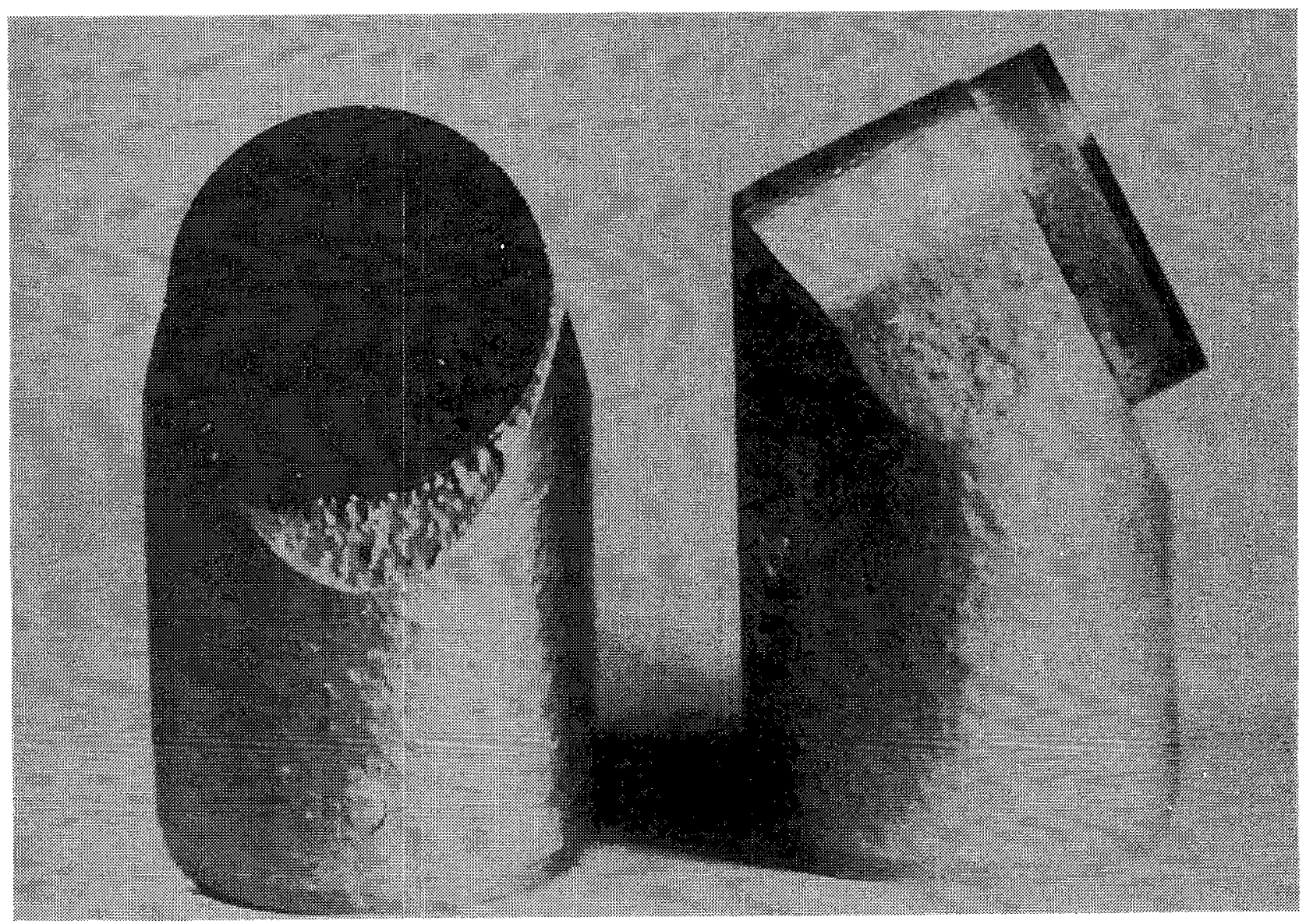

Figure 2 - Single PDC cutter, showing front and side view. Circular diamond compact on this cutter is 0.5 inch in diameter. 
bit lives and penetration rates two to three times those of roller bits in the same wells $[17,20]$. Laboratory tests in formations such as Sierra White granite and Nugget sandstone demonstrated that harder formations accelerate PDC cutter wear, although rapid penetration can be achieved before the cutters wear out $[10,15,16,19]$. Field tests drilling with air in Franciscan graywacke in the Geysers were largely unsuccessful because of problems with cutter studs fracturing off the bit body $[15,17]$. Because of its fractured nature, elevated temperature, and high compressive strength, Franciscan graywacke may be inherently too severe for drilling with WC-Co-backed PDC cutters.

Our later efforts [19-32] have concentrated on developing an analytical understanding of the PDC rock drilling process. Our objectives in these studies have been: (1) to determine the inherent limitations of PDC cutters in various applications, which would help us determine whether the hard-rock geothermal environment is inherently too severe for PDC bits or whether the question of success in drilling long intervals in such applications is merely one of proper design and operation; and (2) to develop procedures for designing PDC bits to operate efficiently and wear evenly in suitable applications .

The development of an analytical model $[22,26,27]$ for predicting cutter wearflat temperatures in a given drilling environment has allowed experimental data $[19,33]$ to be correlated in a manner that indicates a strong effect of wearflat temperature, $\overline{\mathrm{T}}_{\mathrm{w}}$, on cutter wear rates $[27,29]$. The correlation suggests that above a critical temperature of approximately $350^{\circ} \mathrm{C}$, cutters comprised of PDC and WC-Co undergo thermally-accelerated wear. Under these conditions wear rates increase by one or two orders of magnitude, leading to similar-scale reductions in bit life.

Finite element thermal stress calculations of PDC cutters were made in an effort to identify the reasons behind the apparent existence of a critical wearflat temperature $[27,29]$. It was found that at elevated temperatures, several possible wear mechanisms inherent with the PDC-WC-Co combination come into play. When drilling with water or water-based muds, the results indicate the possibility of compressive yielding occuring in the wearflat region due to a combination of thermally-and mechanically-induced stresses. In hard metals such yielding, even on a small scale, causes the development of microvoids in the grain structure, which act as microcrack initiators and lead to increased wear. Furthermore, the thermal expansion coefficient of the PDC and WC-Co is sufficiently different to cause the development of significant tensile stresses along the face of the cutter in the polycrystalline diamond layer. This leads to an increased potential for cracking along the leading face of the cutter. Portions of this face eventually become the cutting edge, where micro-fractures greatly accelerate material wear rates.

When drilling with air, as is sometimes required in geothermal formations due to underpressured conditions, the convective cooling rate of the cutters is greatly reduced, and the temperature distribution is radically changed. The thermal stress field during air drilling places the wearflat under significant tensile stresses, promoting more rapid microfracturing and wear of the WC-Co and PDC [29]. 
The effects of intermittent contact between the cutter and the rock were assessed in order to estimate the effects of conditions such as: 1) pulling the bit off-bottom suddenly before adding a length of drill pipe; and 2) bit bounce caused by drill string dynamics or fractured formations. It was found that thermal shock of the hot wearflat, caused by rapid quenching with the drilling mud, can produce significant tensile stresses in the cutter wearflat region. Fractures initiated in the abrasion process can then be propagated more easily. It is therefore desirable to maintain constant contact between the cutters and the rock surface, and the use of shock subs is recommended to maintain a steady weight-on-bit (WOB) under severe conditions [29]. Results of the finite element analyses also show that when lifting a bit off-bottom, reducing WOB slowly (over a period of about 30 seconds) may have significant advantages over pulling the bit offbottom suddenly. By maintaining some mechanically-induced compressive loading while the cutters slowly cool, thermally induced tensile stresses are suppressed, thereby alleviating some of the wear caused by thermal cycling of the cutters.

The above results have implications for the cutting limitations imposed by the strength of the rock. The thermal model developed in our previous analyses $[22,26,27]$ predicts wearflat temperatures to be directly proportional to the penetrating stress, i.e., the ratio of penetrating force, $F$, to wearflat area, A. There thus exists a critical penetrating stress at which the wearflat temperature reaches $350^{\circ} \mathrm{C}$ and thermallyaccelerated wear begins. This provides an estimate of the upper limit on cutter penetrating stress that can be safely used in a given application.

The minimum penetrating stress that a cutter must impose on a rock surface is related to the strength of the rock. Experimental single-cutter test data indicate that cutter penetrating stresses must reach values on the order of the compressive rock strength before significant penetration of the rock occurs $[20,28,31]$. This suggests that there exists a threshold penetrating stress, equal to the compressive rock strength, that the cutter must impose in order to effectively drill.

A PDC cutter operating envelope between the threshold and critical penetrating stresses has been suggested $[20,28]$. As rock strength increases, this operating envelope shrinks and eventually vanishes. Under these conditions, PDC bits cannot achieve significant rock penetration without experiencing thermally-accelerated wear, and bit life is virtually non-existent. The temperature and wear models that have been developed are useful in estimating these wear limitations under different combinations of downhole conditions. Knowledge of these limitations is helpful in bit design because it can be used to help optimize various design parameters, such as bit profile and cutter placement, for different applications.

Use of the temperature and wear models in bit design requires an estimate of cutter penetrating forces during drilling. If an accurate model of cutter forces is available, the following bit performance parameters can also be determined by summing forces and moments acting on the bit: weight on bit, drilling torque, side force, and bending moments. Optimal placement of cutters on the face of a bit to promote uniform cutter wear and to balance the loads and prevent bit wobble should then be possible. 
This report deals with the development of a model for estimating cutter forces and the development of a computer code that implements the model to provide an analytical tool for general PDC bit design. The use of the computer program is demonstrated, and some general conclusions derived from its use are identified and discussed.

The potential for using moderate-pressure waterjets to extend the range of application of PDC cutters is also explored. Previous work by Hood $[34,35]$ and Dubugnon [36] with large WC-Co cutters used in hard-rock mining applications has shown that cutter forces can be significantly reduced if waterjets are directed onto the rock surface immediately ahead of the cutter. Penetrating force reductions of 508 with 2500 psi jets and $75 \%$ with 9,000 psi jets have been reported. Our previous analysis [28] indicates that if such reductions could also be achieved with PDC cutters, these cutters could operate under more severe conditions and in significantly harder rocks, thereby extending the applicability of PDC bits to more types of formations. This report presents new data obtained with PDC cutters and cavitating jets that support that conclusion. The application of the cutter/waterjet technique to PDC bit design is also discussed. 


\subsection{DEVELOPMENT OF A PDC CUTTING FORCE MODEL}

We seek a model of the PDC cutting process that will allow us to determine the penetrating and drag forces acting on each cutter located on the bit face. The primary parameters that affect these forces include the rock type, cutter design and wear state, position on the bit, cutter interaction, cutting speed, rock stress state, and fluid environment. Some of these parameters are illustrated in Figure 3.

It is possible to duplicate many of these parameters in laboratory single-cutter tests. Most of the cutting conditions encountered by a single cutter in full-scale, atmospheric-pressure, laboratory bit tests can be duplicated by using a modified milling machine and relatively small rock samples. Similarly, deep-hole drilling conditions can, to a large degree, be duplicated in single-cutter tests in the laboratory. Test cells exist that measure single-cutter forces under elevated hydrostatic, confining, and pore pressures [37-40]. Typical downhole rock types, cutter configurations, and cutting speeds can also be duplicated with these test cells.

A more difficult simulation is the cutter interaction that occurs on full-scale bits, where cutting forces on each cutter are reduced by the presence of previous cuts made by adjacent cutters. We have found in unconfined rock tests at atmospheric pressure that interaction does not occur unless the previous cuts are laterally close enough that they actually remove rock that would otherwise be removed by the test cutter. The crosssectional area of rock removed by each cutter, therefore, seems to be the parameter that characterizes cutter interaction and controls cutter forces.

Our experience with single-cutter tests is that for reasonable depths of cut, PDC cutters do not cause much rock breakage outside the projected area of the cutter profile. Even in coarse-grained and brittle rock such as Sierra White granite at atmospheric pressure, the profile of a cut made with a PDC cutter closely matches the profile of the cutter itself. An even closer match between cut and cutter profiles should be obtained under the elevated hydrostatic and confining stresses found downhole, since a transition from brittle to more ductile rock breakage mechanisms occurs $[37,41]$.

Shown in Figure 4 is a typical sequence of cutting edge profiles for several sharp (i.e. unworn) cutters on the leading flat face of a PDC bit. These profiles are obtained during two bit revolutions as the cutters pass through a radial plane containing the longitudinal axis of the bit. The shaded areas represent the steady-state cross-sectional areas of cut for each cutter. Since the centers of the cutters are all assumed to lie in the same plane normal to the longitudinal axis of the bit, the downward vertical displacement of one cutter profile relative to a preceding one is due entirely to the advancement of the bit as it rotates. The shapes of the cross-sectional areas of cut are seen to be quite complex, even for simple bit geometries. In an arbitrary bit design, the cross-sectional areas of cut are functions of the radial, circumferential, and longitudinal distribution of cutters on the bit face as well as the wear state of the cutters and the penetration rate of the bit. Although it is possible to duplicate some interaction patterns in the laboratory [40], it is clearly 


\section{SIDE VIEW}

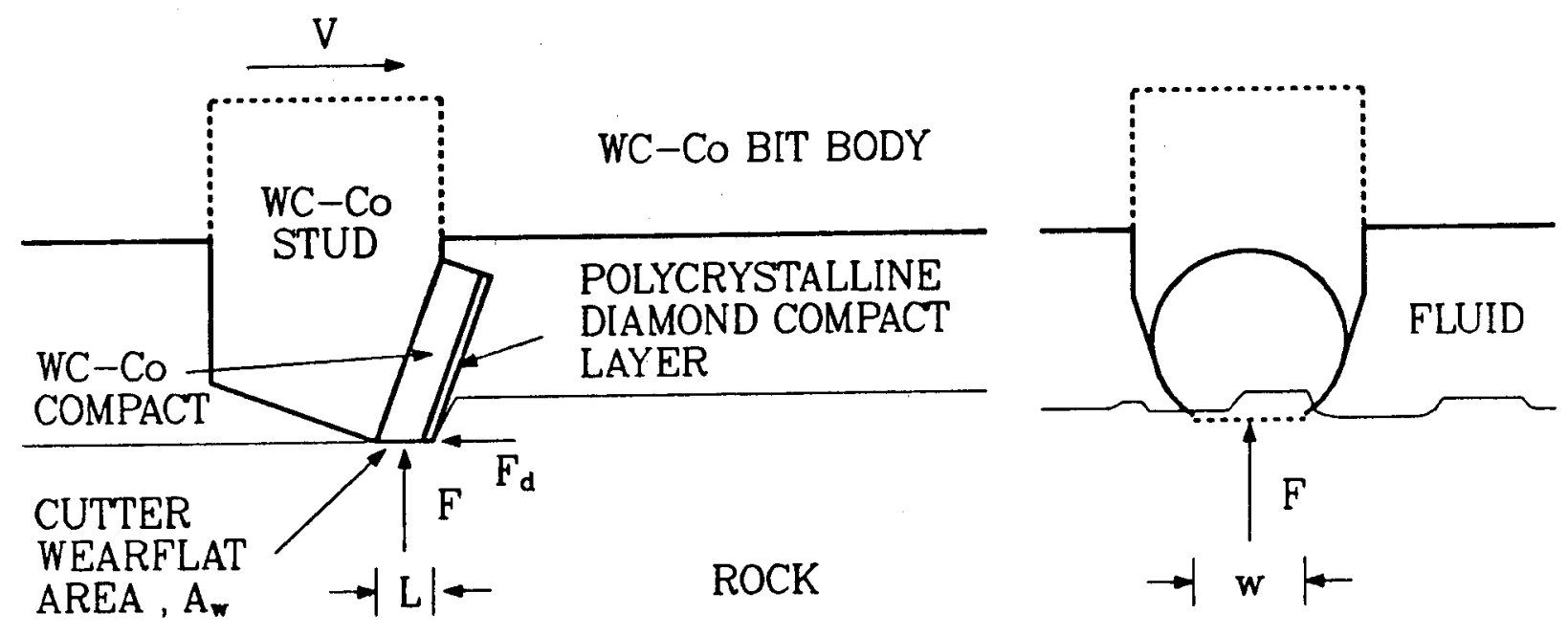

Figure 3 - Schematic of a single PDC cutter mounted on the leading face of a bit and experiencing interaction with nearby cutters. 


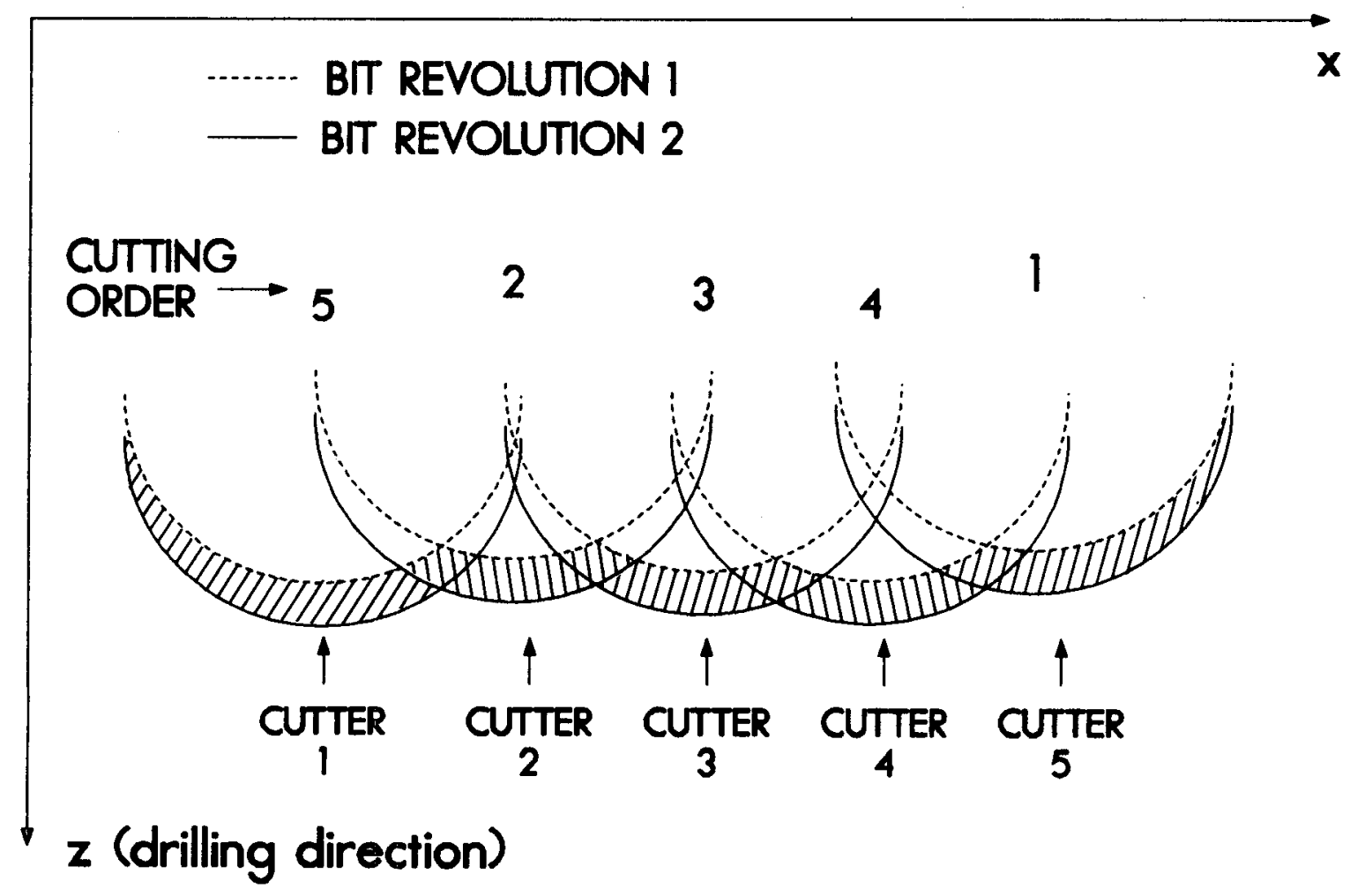

Figure 4 - Cutting edge profiles for sharp cutters on the leading face of a PDC bit. Shaded areas represent cross-sectional areas of cut. 
impractical in single-cutter tests to duplicate all the degrees of interaction that could be experienced by cutters in a given bit design.

We, therefore, seek to separate the effects of cutter interaction from the effects of intrinsic drillability of the cutter/rock combination under the imposed conditions. Intrinsic drillability under any set of laboratory conditions can then be determined by conducting single-cutter tests using a standard cut geometry; and an analytical model can be employed to account for cutter interaction.

We choose the simplest geometry possible for the standard cut geometry, that of a single cut on a flat rock surface with no adjacent cuts that might interact with the test cut. The depth to which the cutter penetrates the flat rock surface is a function of the stresses imposed in the rock by the cutter. In the case of worn cutters, the penetrating stress is defined as the average normal stress imposed on the rock surface; thus

$$
F / A_{w}=f \operatorname{ct}(\delta) .
$$

In the case of a sharp cutter, the wearflat area is very close to zero. Yet a finite penetrating force is still required to cause penetration of the rock [31]. The reason for this is that the crushed rock zone directly beneath the cutting edge acts to distribute the force over a finite area. For such cases, we seek in our tests to simply define the functional dependence,

$$
F=f \operatorname{ct}(\delta) .
$$

The following subsections describe the procedures used to obtain experimental data that define Eqs. 1 and 2, and a method is developed for using these correlations to determine cutter performance during interacting cuts.

\subsection{Experimental Set-up. Procedures, and Conditions}

Rock-cutting tests were conducted in three rock types with PDC cutters having various amounts of wear. Berea sandstone, Tennessee marble, and Sierra White granite were used in order to cover a wide range of rock properties such as strength, composition, and ductility.

Berea sandstone is a relatively soft rock with a measured uniaxial compressive strength, $\mathrm{S}_{\text {, }}$ of $7,100 \mathrm{psi}$ [31]. Tennessee marble, also known as Holton limestone, is a much harder rock, with $\mathrm{S}=17,800$ psi. Sierra White granite, $S=21,500 \mathrm{psi}$, is somewhat harder than the marble, but its composition is quife different. The granite has a high quartz content (39\% by weight) and thus causes cutters to wear at a higher rate. 
Two milling machines were used in the test program. The end-mill shown in Figure 5 was used for obtaining quantitative data. The fixed head on the mill was instrumented with a triaxial force transducer, to which the cutter was mounted. Rock samples ranging in length from 12 to 18 inches and in width from 5.0 to 9.75 inches were placed on the milling table and secured with clamps. Linear, parallel cuts in the top surface of a rock sample were made by moving the table at a fixed speed of $2.2 \mathrm{in} / \mathrm{sec}$, the table's maximum speed. Cuts were made in the rock samples under dry cutting conditions at depths ranging from 0.010-0.100 inch, which covers the range generally encountered by a single cutter on a bit downhole.

The milling machine table was later fitted with a steel enclosure and a waterjet nozzle holder mounted ahead of the cutter. A profile of the nozzle holder and and its relationship to the cutter are shown in Figure 6 . The nozzle holder was designed so that the nozzle standoff distance, d, inclination angle, $\phi_{n}$, and jet impingement distance, $d_{j}$, could be adjusted over limited ranges. Only one set of these parameter's was used in the test program currently reported: $d_{s}=1.6$ inches, $\phi_{n}=45^{\circ}$, and $d_{j}=0.1$ inch. These values were chosen because they represent practical vatues that might be used in an actual PDC bit design.

The nozzles used in the test program were self-resonating, cavitating jet nozzles designed and fabricated by Tracor Hydronautics, Inc. under contract to Sandia National Laboratories. These nozzles are the product of several years of research aimed at optimizing nozzle designs that erode rock effectively using drilling mud pressures available on conventional drill rigs (<5000 psi) [42-46]. They are designed to produce jets that cavitate under higher ambient pressures, and hence at greater hole depths, than conventional nozzles. The cavities produced in these jets are created by vaporization of the liquid in the center of high velocity vortices that form in the jet shear zone. When these cavities collapse near a rock surface, high-speed microjets impact the rock surface with pressures of up to 200,000 psi [47], substantially increasing the rock erosion rate. Two nozzles were fabricated for the single-cutter tests: one optimized for a pressure drop of $2000 \mathrm{psi}$ and the other for a pressure drop of $4500 \mathrm{psi}$. Both had orifice diameters of 0.1 inches. A more complete description of the specific nozzles fabricated for this test program is given in Ref. 46. Cuts made with PDC cutters assisted by the high-pressure waterjets produced with these nozzles were compared with identical cuts made with 80 psi waterjets and cuts made with no jets at all.

A computerized data acquisition system was used to sample three components of cutter force during a cut on the rock sample: a vertical or penetrating force, a horizontal drag force, and a horizontal side force. The nominal sampling rate of $75 \mathrm{~Hz}$ allowed several hundred samples of each force channel to be made for each cut. At the end of each cut, the computer averaged the data and recorded the forces along with other pertinent test data on floppy disk. These other data include rock type, cutter wearflat area, depth of cut, lateral distances to adjacent cuts, and their depths.

Cutters in various stages of wear were used in an effort to determine the effects of wear on cutter forces. New PDC cutters with sharp edges were 


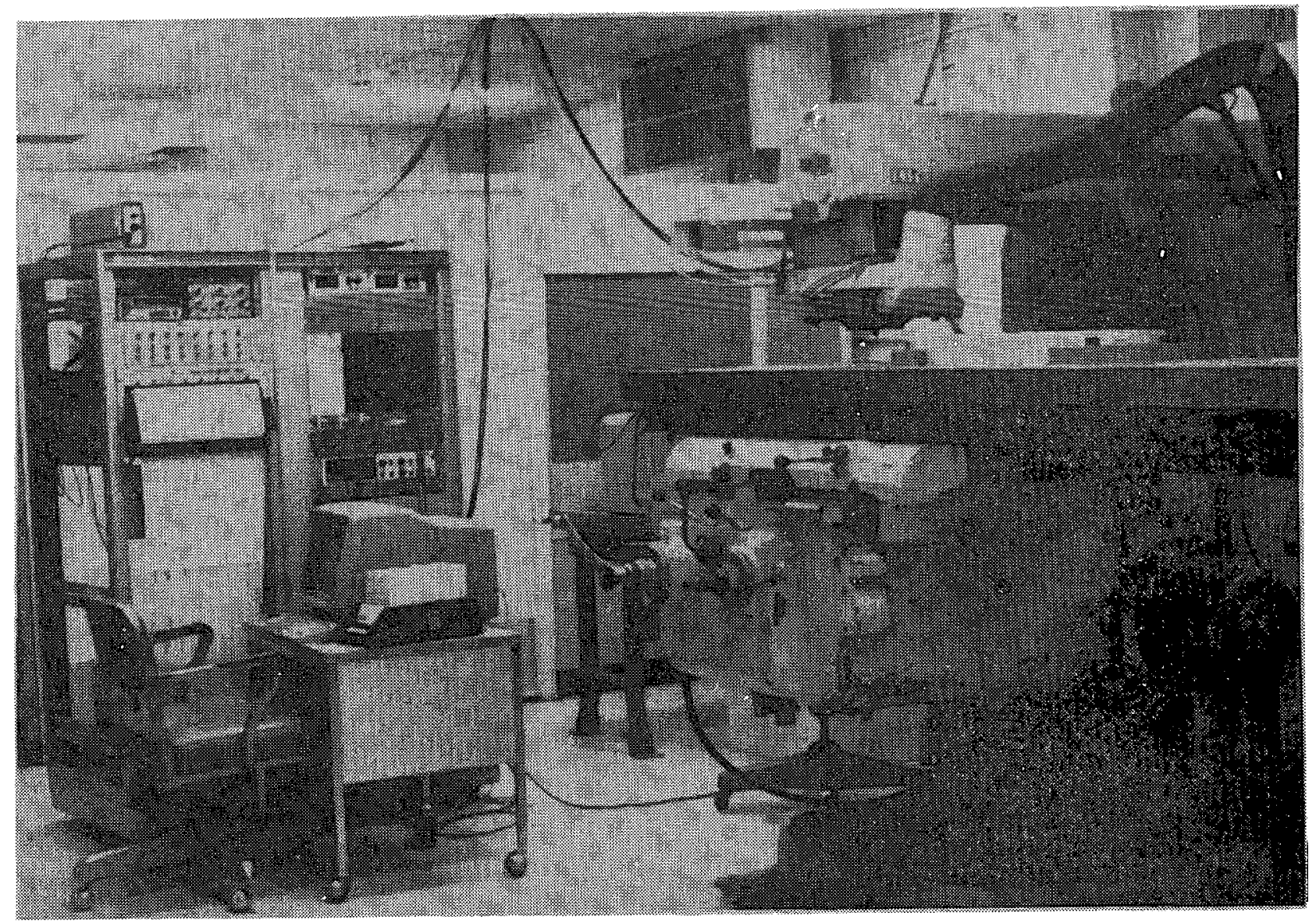

Figure 5 - Milling machine used for linear single-cutter tests. 


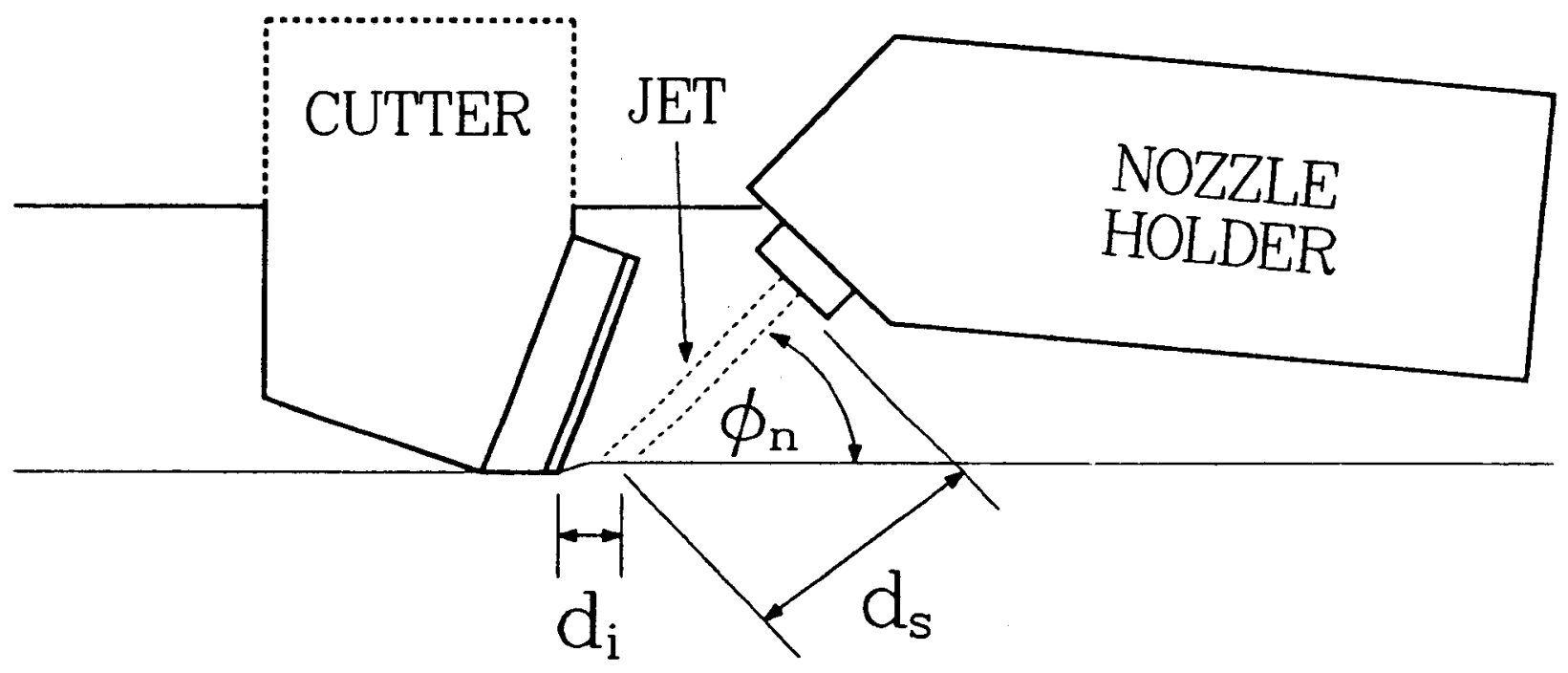

Figure 6 - Geometry of single-cutter tests with waterjet assistance. 
used, as were cutters with machine-ground, laboratory-worn, and field-worn wearflats. The machine-ground wearflats were produced by grinding new cutters in a direction parallel to the direction of cutter travel. Cutters with field-worn wearflats were removed from commercial bits that had been run in oil and gas wells.

Laboratory-worn wearflats were obtained with the vertical milling machine, as shown in Figure 7. A cubical specimen of Sierra White granite, $3 \mathrm{ft} \times 3 \mathrm{ft} \times 3 \mathrm{ft}$, was placed on the mill table. The cutter/dynamometer system was attached to a moving head on the vertical mill that traversed the top face of the rotating rock specimen. Because of the slow maximum feed rate of the traversing head (.06 inch/table revolution), the resulting spiral cuts had significant overlap. This provided a means for rapidly wearing the cutters in the laboratory.

Cutter wearflats were measured as follows. A piece of carbon paper was placed between two sheets of plain paper, and the three sheets were then placed between the cutter and the flat rock surface. The cutter was then vertically loaded to a static penetrating force level typical of the level encountered in cutting tests with the same cutter and rock. The portion of the cutter wearflat that contacted the rock left a carbon impression on the plain paper. This impression was overlain with a fine rectangular grid (1 $\mathrm{mm} \times 1 \mathrm{~mm}$ ) and the area calculated by counting shafed blocks. Cutters with measured wearflats ranging from 0.016 to 0.040 in $^{2}$ were tested in this study. Cutter wearflat characteristics are listed in Tables A-1 through A-7 (Appendix A).

Two sizes of cutter were tested. Most cutters had compact diameters of 0.5 inch, the size that has been traditionally used in PDC bits. Also tested, both new and laboratory-worn, was a cutter having a compact diameter of 0.75 inch. All cutters had backrake angles of $20^{\circ}$.

Finally, two types of cut were used: non-interacting cuts and interacting cuts. Non-interacting cuts, as illustrated in Figure 8 , are cuts where the lateral spacing, d, between cuts is large enough that forces in a given test cut are not affected by the presence of previous, laterallyadjacent cuts. It was found that previous cuts do not affect the forces in a given test cut unless those previous cuts remove rock that otherwise would be removed in the test cut.

The geometry of interacting cuts designed to simulate the interaction typical of PDC bits is illustrated in Fig. 9. The test cut here is the 0.080 -inch cut, which was made after the adjacent 0.040 -inch cuts. The interaction shown in the symmetric case, for example, would be encountered with a bit on which two cutters near the same radial location are circumferentially located $180^{\circ}$ from the test cutter. In the one-half rotation of the bit between the time the adjacent cutters pass through a given radial plane and the time the test cutter passes through the same radial plane, the bit advances so that the test cut is at a lower level in the rock. A wide range of interaction was achieved by varying the lateral distance, $d$, over a range of 0.050 to 1.25 inches, in both the symmetric pattern (two adjacent cuts) and the asymmetric pattern (one adjacent cut). 


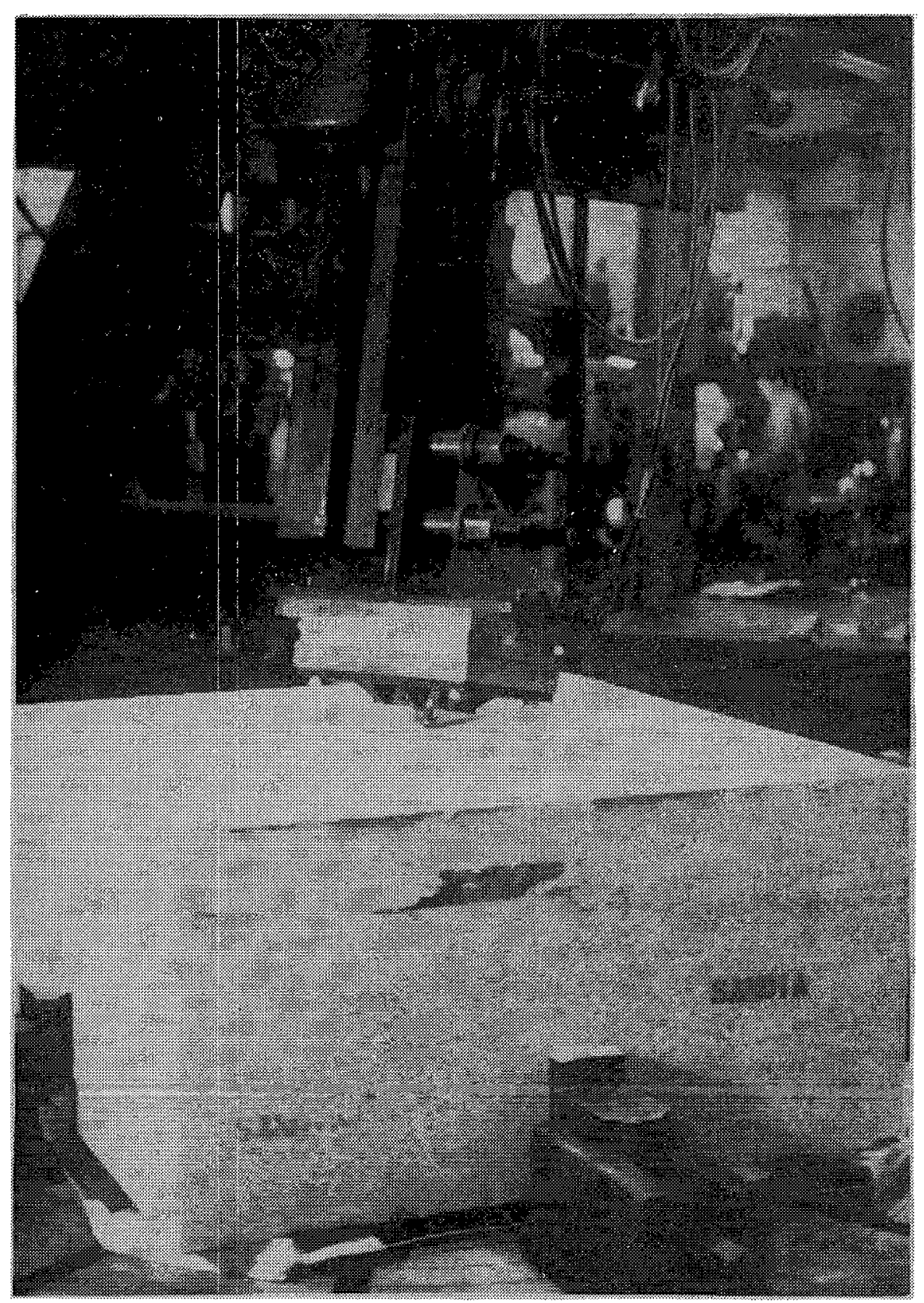

Figure 7 - Vertical milling machine used for wearing PDC cutters in the laboratory. 


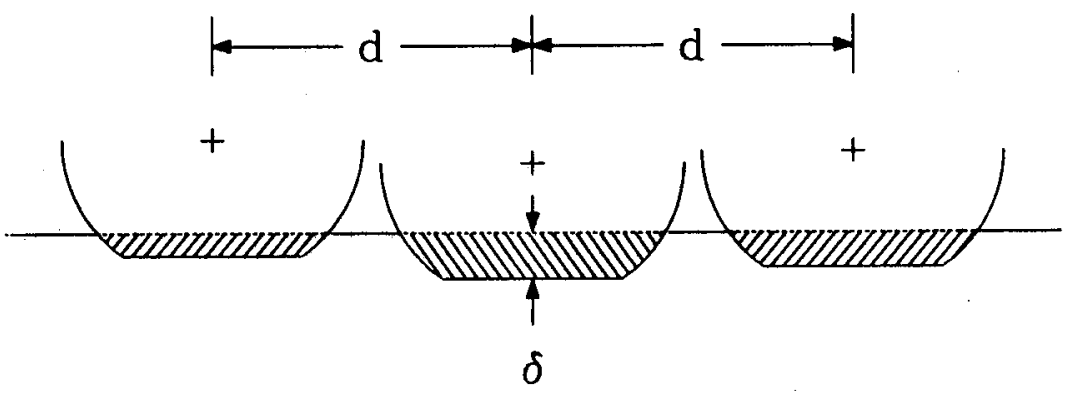

Figure 8 - Cutting pattern used in non-interacting single-cutter tests. Shaded areas are cross-sectional areas of cut. 


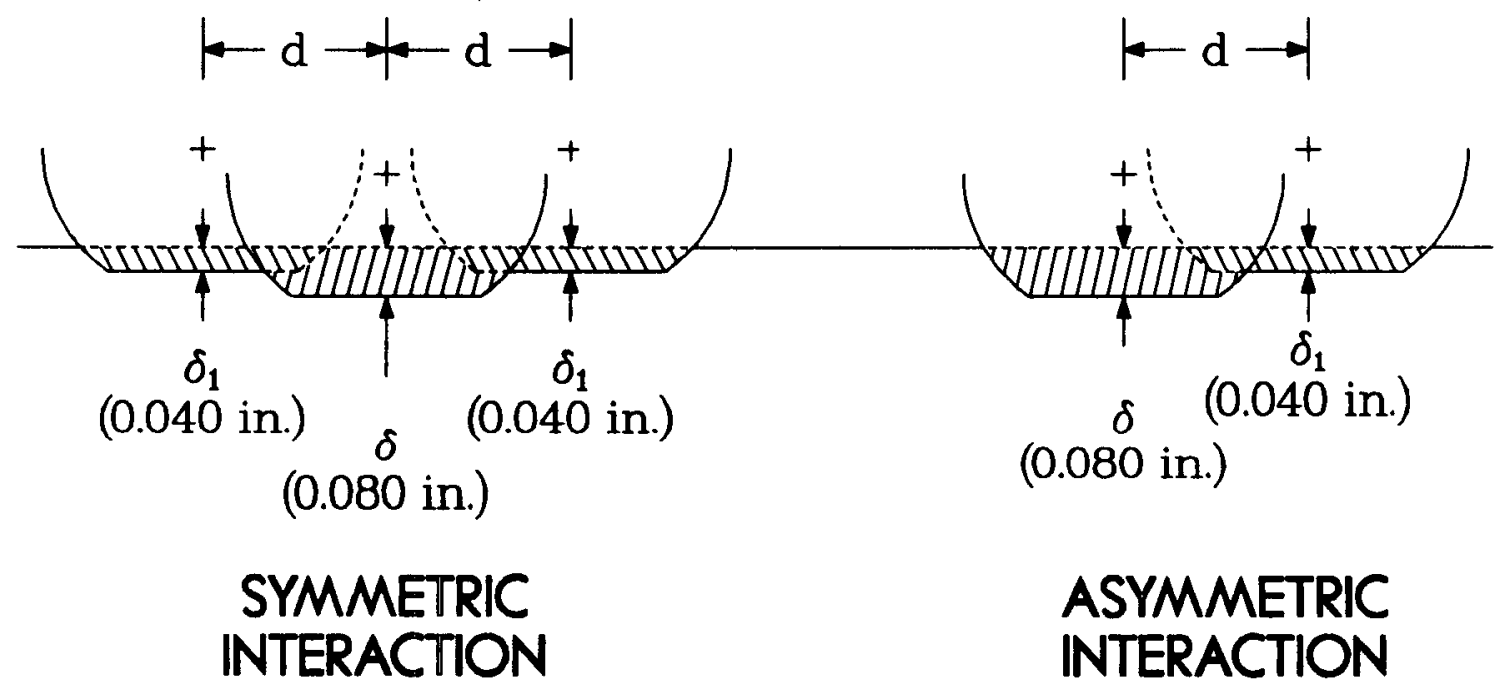

Figure 9 - Cutting patterns used to simulate cutter interaction in singlecutter tests. Test cut is the 0.080 -inch cut in each pattern. 


\subsection{Test Results: Dry, Non-Interacting Cuts}

Penetrating and drag forces measured in dry, non-interacting cut tests with the various cutters and rock types are tabulated in Tables A-2 through A-4 (Appendix A).

Shown in Figures 10 and 11 are the measured penetrating stresses, $F / A$ plotted as a function of depth of cut for the worn cutters. The behavior of the data for each rock type suggests correlations of the form

$$
\mathrm{F} / \mathrm{A}_{\mathrm{W}}=\mathrm{C}_{1} \delta^{\mathrm{n} \boldsymbol{l}}
$$

where the constants $C_{1}$ and $n 1$ are determined from a least squares $f i t$ of the data in $\log -\log$ space. The curves drawn in Figures 10 and 11 show the values of $C_{1}$ and $n 1$ calculated for each rock type. This simple form of correlation equation provides an adequate fit of the data.

The most significant result is that all the data for a given rock type collapse to approximately the same curve, regardless of the size or shape of the wearflat or the diameter of the cutter compact. This suggests two important conclusions:

(1) The penetrating force on a worn cutter for a given depth of cut is directly proportional to the wearflat area. This implies that for a given rock and set of operating conditions the values of $\mathrm{C}_{1}$ and nl can be determined with any cutter having a measureable wearflat, and those values should also be valid for other stages of wear with the same type cutter.

(2) For a given depth of cut and a given wearflat area, a large worn cutter requires no greater penetrating force than a small cutter, yet the large cutter does remove more rock. This suggests an improved cutting efficiency with increased cutter size, at least within the range of cutter sizes considered.

The results shown in Figures 10 and 11 for Sierra white granite and Tennessee marble confirm that for these hard rocks, penetrating stresses on the order of the compressive rock strength must be imposed on the rock surface before significant penetration of the rock occurs. This supports the idea of a threshold penetrating stress discussed earlier and suggests that the penetrating force component is important in crushing the surface of hard rocks, leading to cutter pentration. With the softer, more plastic Berea sandstone, however, significant rock penetration can be achieved with penetrating stresses significantly lower than the compressive rock strength. This suggests that crushing of the rock surface is less important with soft rocks and that the drag force component plays a greater role in machining, rather than crushing, the rock surface. 


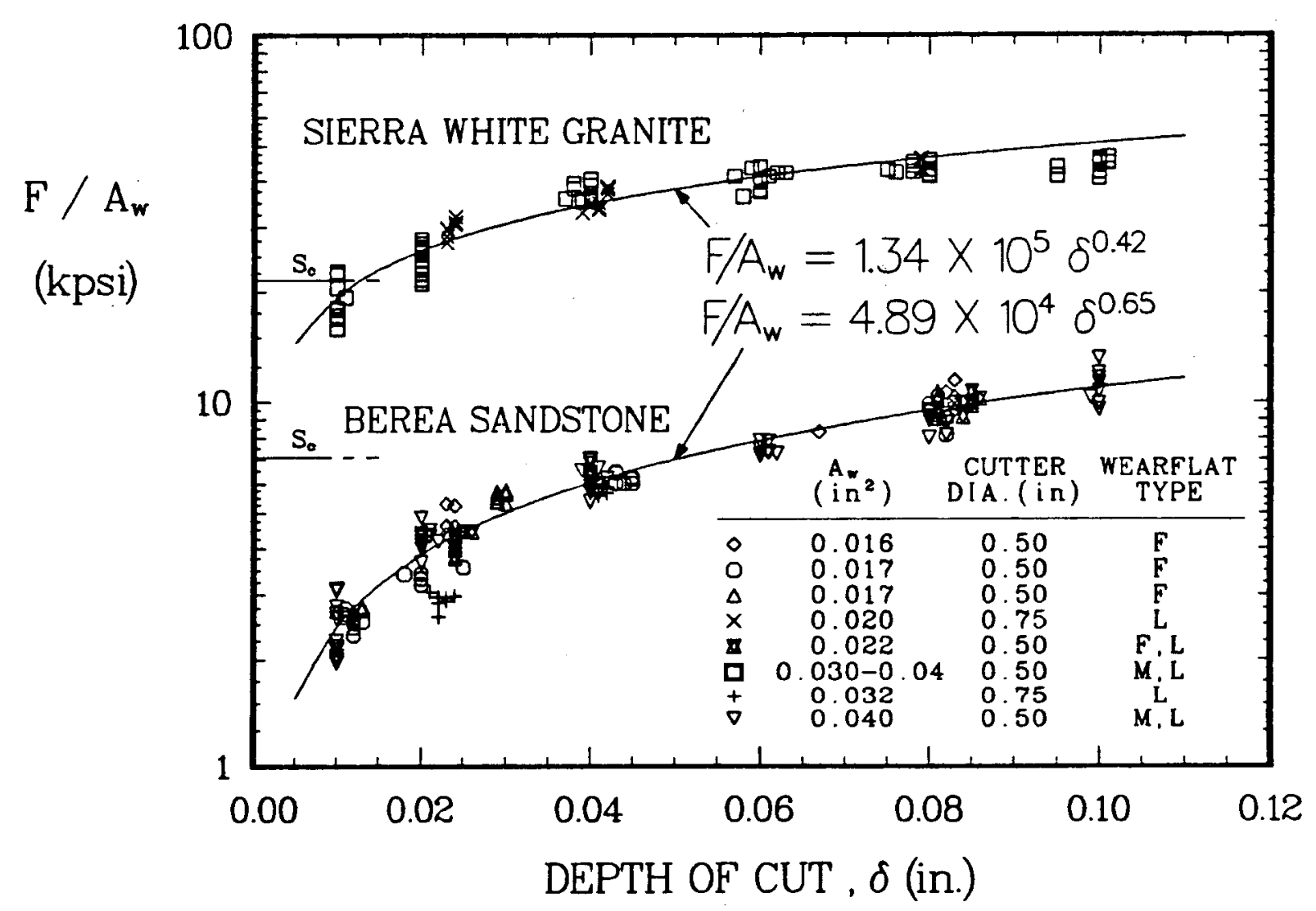

Figure 10 - Measured penetrating stresses with various wearflat
configurations in dry, non-interacting cuts. Wearflat type:
F=field worn; $L=1$ aboratory worn; M=machine ground. 


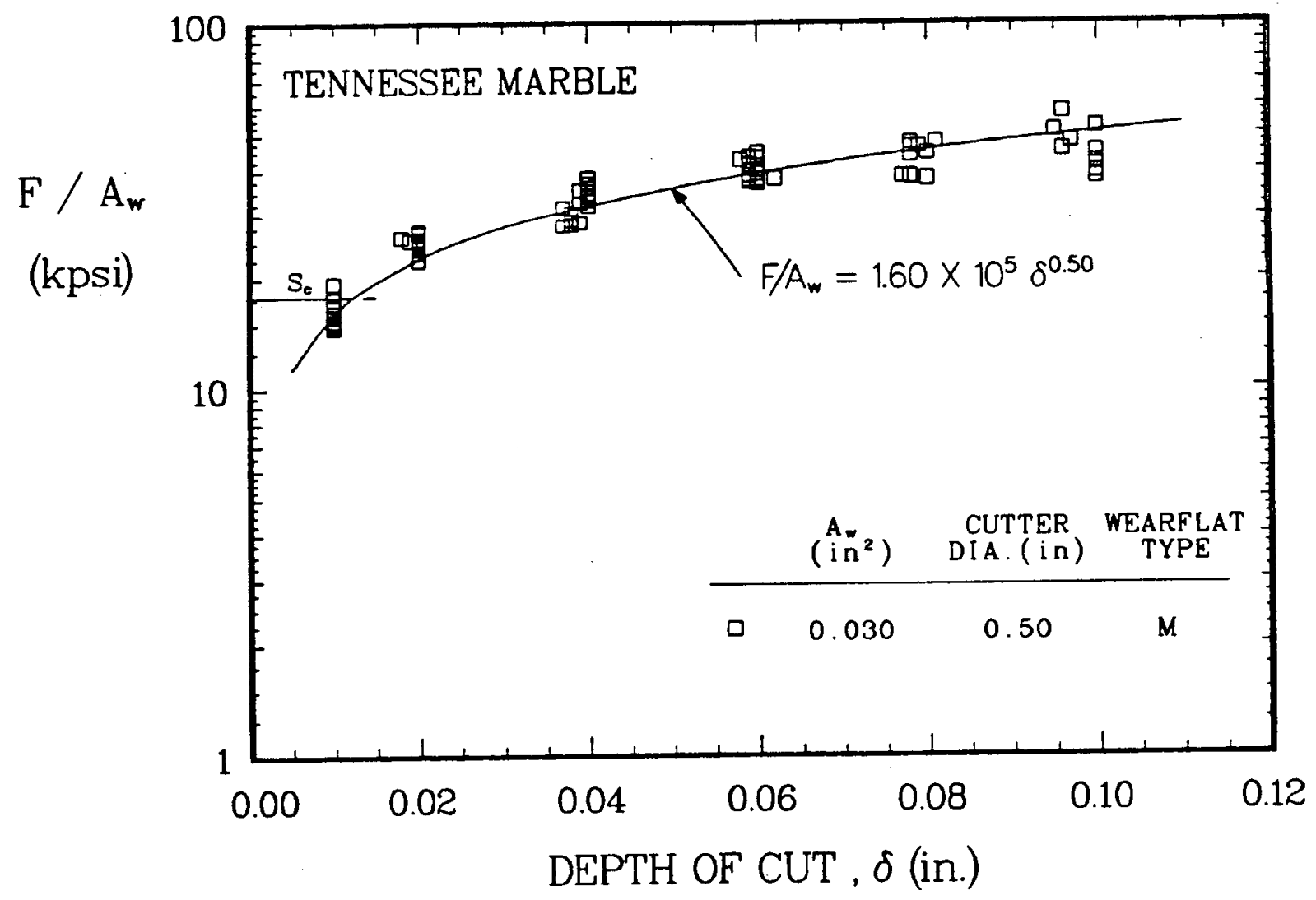

Figure 11 - Measured penetrating stresses with various wearflat configurations in dry, non-interacting cuts. Wearflat type: $\mathrm{F}=\mathrm{field}$ worn; L=laboratory worn; M=machine ground. 
Penetrating forces measured with sharp cutters in dry, non-interacting cuts are shown in Figure 12. Correlations of the form

$$
F=c_{2} \delta^{n 2}
$$

were determined, and the resulting values of $\mathrm{C}_{2}$ and $\mathrm{n} 2$ are shown in Figure 12. These data indicate another surprising result: even in the sharp condition, the larger cutter requires no larger penetrating force than the smaller cutter. In fact, in the tests with Berea sandstone at 0.08 inch, the larger cutter actually required slightly lower penetrating forces than the smaller cutter.

The cutter drag forces, $F_{\text {, }}$, measured in the dry, non-interacting cuts with worn cutters are shown in Figures 13 and 14 , plotted as a ratio with the penetrating force, $F$. We define this ratio as the cutter drag coefficient,

$$
\mu_{d}=F_{d} / F \text {. }
$$

We see that the drag coefficient is a function of the rock type, but it is relatively independent of the depth of cut and wearflat area. If a model is available to predict $F$, it is then possible to estimate $F_{d}$ :

$$
F_{d}=\mu_{d} F
$$

where, for instance, $\mu_{d}=0.64$ for worn cutters in Sierra white granite and Tennessee marble and $\mu_{d}=0.95$ in Berea sandstone. The higher drag coefficient for Berea sandstone agrees with the conclusion that the drag force component is more important with this rock type than with the harder rock types.

Drag coefficients for sharp cutters are shown in Figure 15. When compared with the results for worn cutters, as represented by the curves, it is seen that sharp cutters have consistently higher drag coefficients. The larger cutter has slightly higher drag coefficients that the smaller cutter in deep cuts.

\subsection{Test Results: Non-Interacting Cuts with Waterjet Assistance}

Shown in Figure 16 are the penetrating stresses measured with waterjets impinging on the rock surface ahead of the cutter. These data are tabulated in Table A-5 (Appendix A). Note that the stresses obtained with 80 psi jets are similar to those measured in the dry cuts, as represented by the upper curve in Figure 14. The jet velocity with this nozzle pressure is not sufficient to affect penetrating stresses, and the simple presence of water at the cutter/rock interface also seems to have little effect. At elevated 


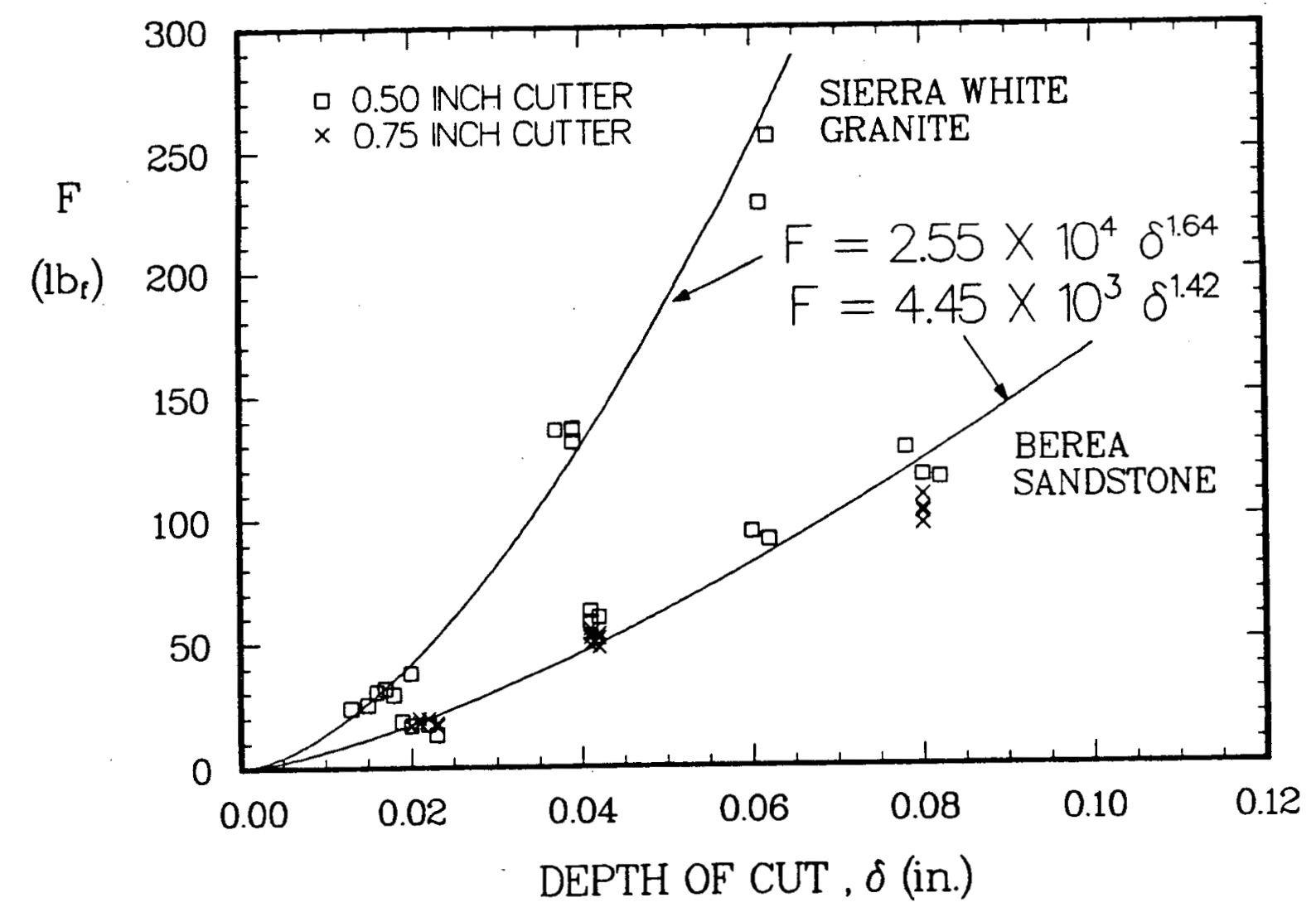

Figure 12 - Measured penetrating forces with sharp cutters in dry, noninteracting cuts. 


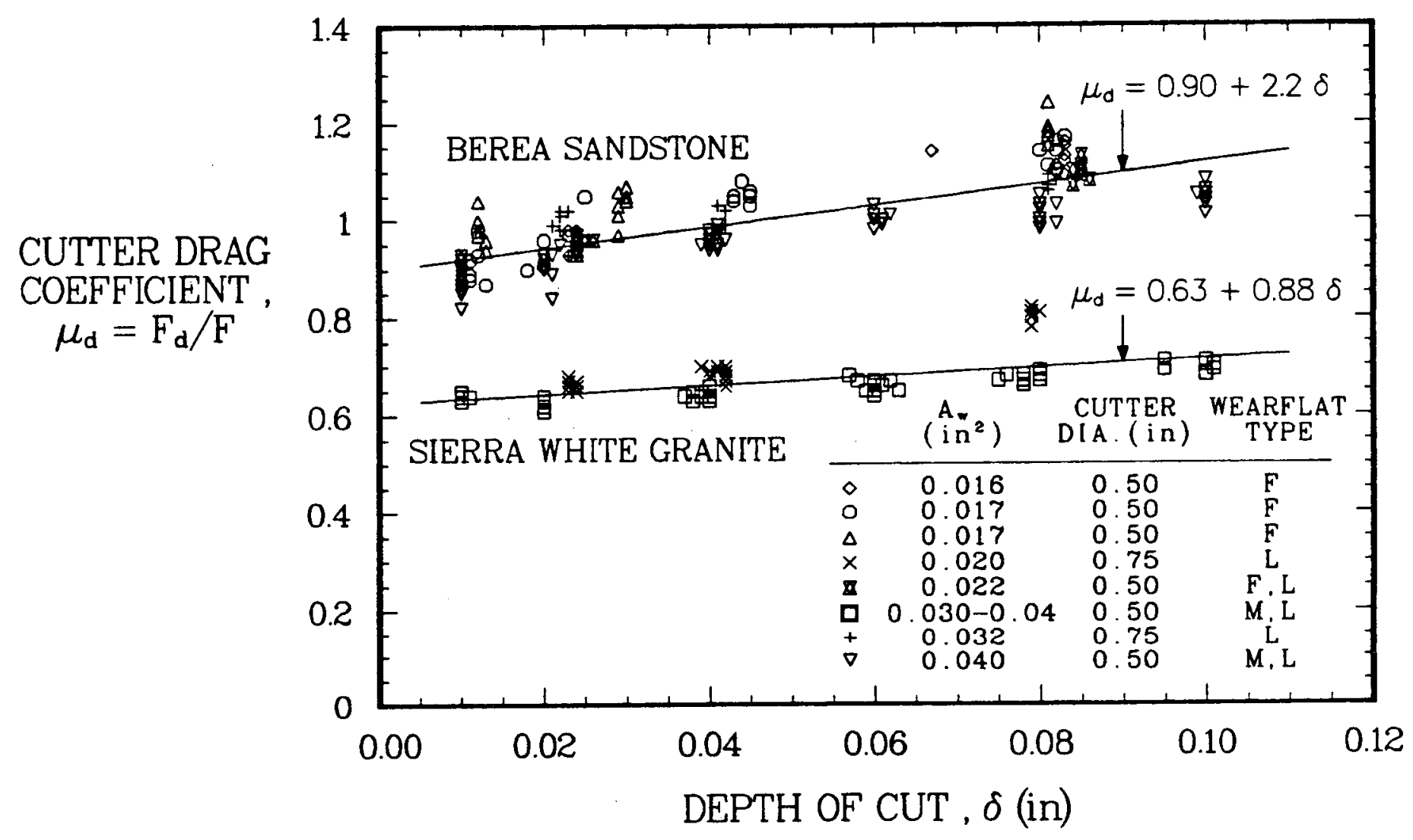

Figure 13 - Measured drag coefficients with various wearflat configurations in dry, non-interacting cuts. Wearflat type: F=field worn; $\mathrm{L}=1$ aboratory worn; $\mathrm{M}=$ machine worn. 


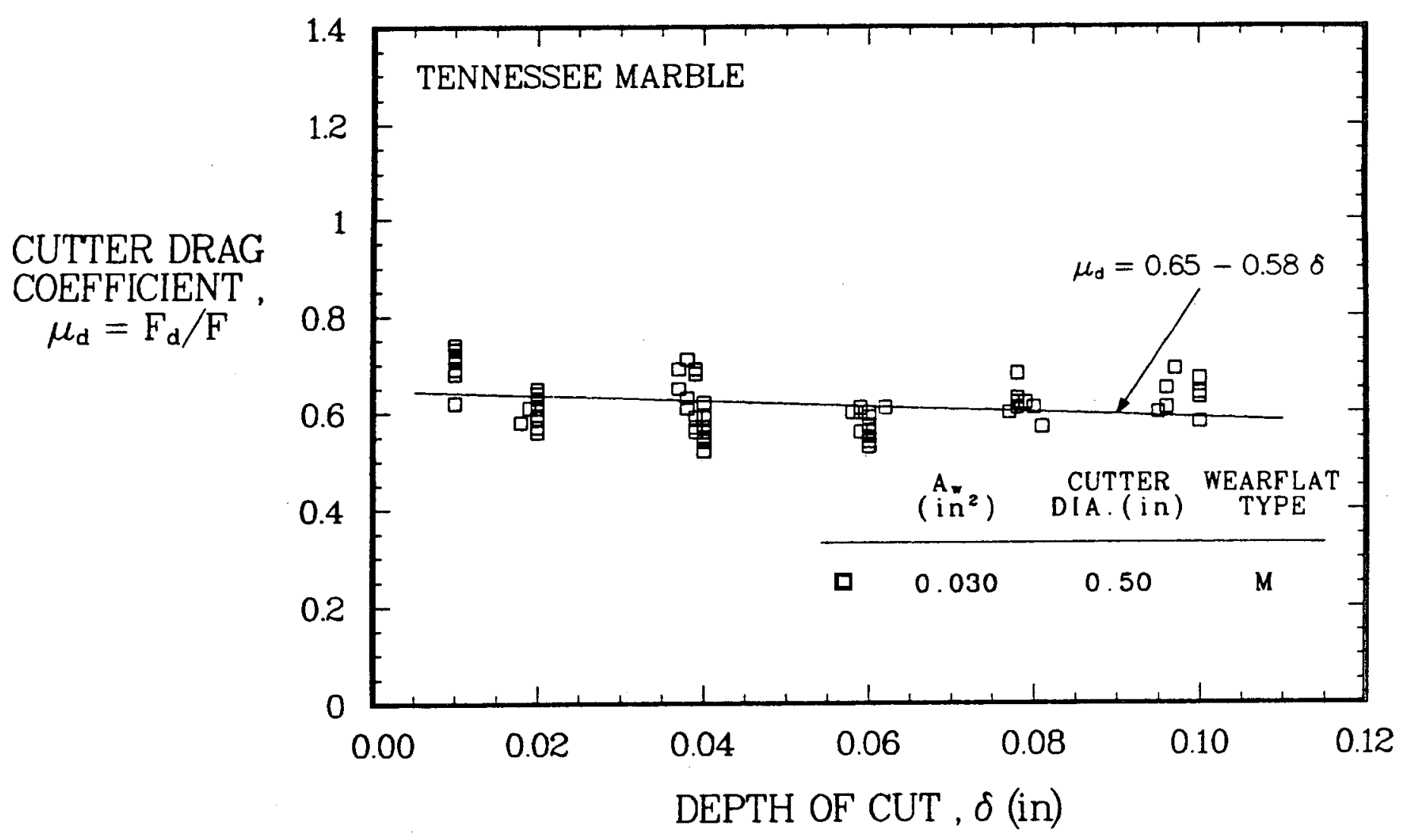

Figure 14 - Measured drag coefficients with various wearflat configurations in dry, non-interacting cuts. Wearflat type: F=field worn; $\mathrm{L}=1$ aboratory worn; $\mathrm{M}=$ machine worn. 


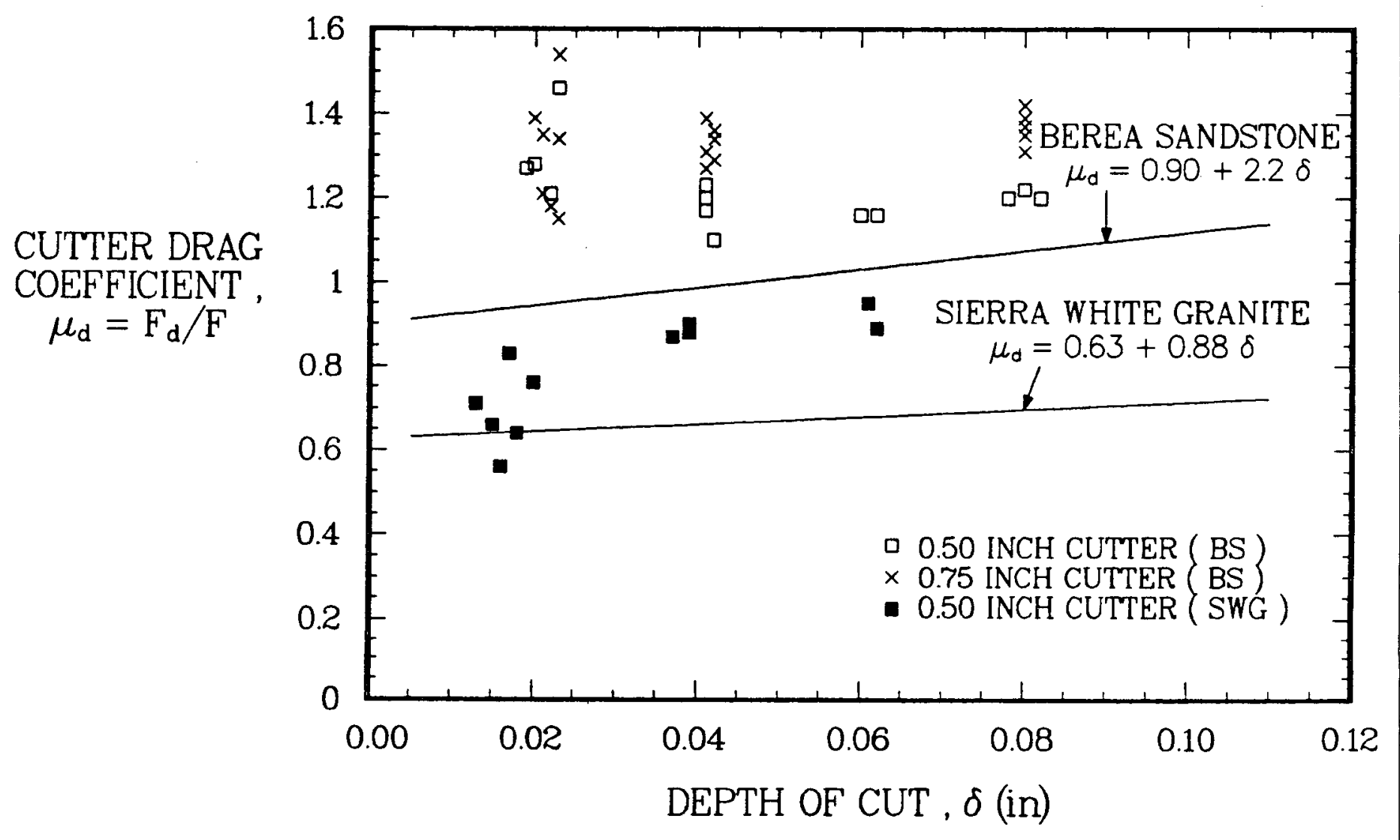

Figure 15 - Measured drag coefficients with sharp cutters in dry, noninteracting cuts. Curves represent mean data obtained with worn cutters (Fig. 13). 


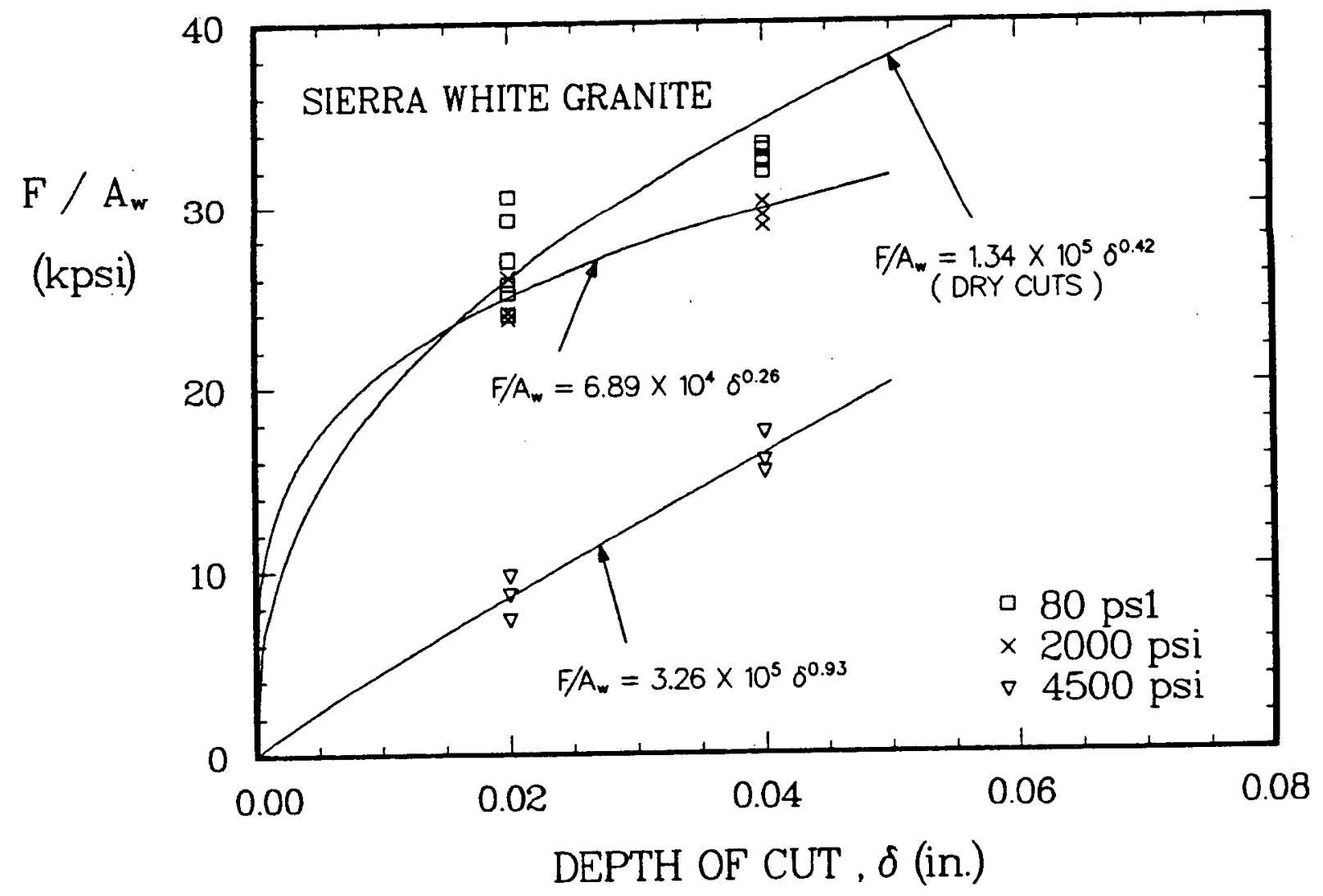
Figure 16 - Measured penetrating stresses in non-interacting cuts made with
waterjet assistance. Upper curve represents mean data from dry cuts (Fig. 10). 
nozzle pressures, however, penetrating stresses are significantly reduced. With jet pressures of $2,000 \mathrm{psi}$, the penetrating stresses required to cut Sierra White granite to a given depth are reduced by 10 to 158 . With assistance from 4,500 psi waterjets, cutter penetrating stresses are reduced by 50 to 658 .

Passes made over the rock surface without actual contact between the cutter and the rock revealed that the 2,000 psi jet alone did not cause visible damage to the rock surface. The observed reductions in penetrating stresses in this case are probably due to improved cleaning at the cutter/rock interface. Efficient removal of cutting fines helps maintain greater stress concentration in the rock at the cutting edge.

In contrast, the 4,500 psi jet alone caused considerable damage to the rock surface, even though the nozzle standoff distance was greater than 1.5 inches. Though not a continuous cut, the path left by the jet in the granite resembled a series of closely-spaced irregular holes, some of which were up to 0.08 inch deep. The assistance given to the cutter by the jet in this case was due in large part to a reduction in the cutter cross-sectional area of cut and in the strength of the remaining rock surface.

The drag coefficients measured with the jet-assisted, non-interacting cuts are shown in Figure 17, where they are compared with the results for the dry cuts. The drag forces are seen to be reduced by the presence of water, but they are not greatly affected by jet pressure. This suggests that the reduction in drag coefficient is caused by the lubrication effect of water, which reduces the friction component of the drag force. This supports a model for the drag force which considers the force to be the sum of two components [29]:

$$
F_{d}=F_{c}+F_{f},
$$

where $F$ is the cutting force and $F_{f}$ is the friction force. We may divide Eq. 6 by ${ }^{C} F$ and recognize that the friction coefficient, $\mu$, is defined as the ratio $\mathrm{F}_{\mathrm{f}} / \mathrm{F}$. The result is then

$$
\mu_{d}=F_{c} / F+\mu .
$$

We thus see that a reduction in friction coefficient results in an equal reduction in drag coefficient.

Measured friction coefficients between PDC cutters and Sierra White granite are approximately 0.16 under dry cutting conditions and 0.07 when cutting with low-pressure waterjets directed at the cutter/rock interface [19]. The difference in friction coefficient of about 0.09 between the wet and dry cutting conditions is apparently translated to a comparable difference in drag coefficient between the two conditions, as predicted by Eq. 7 and confirmed in Figure 17 . 


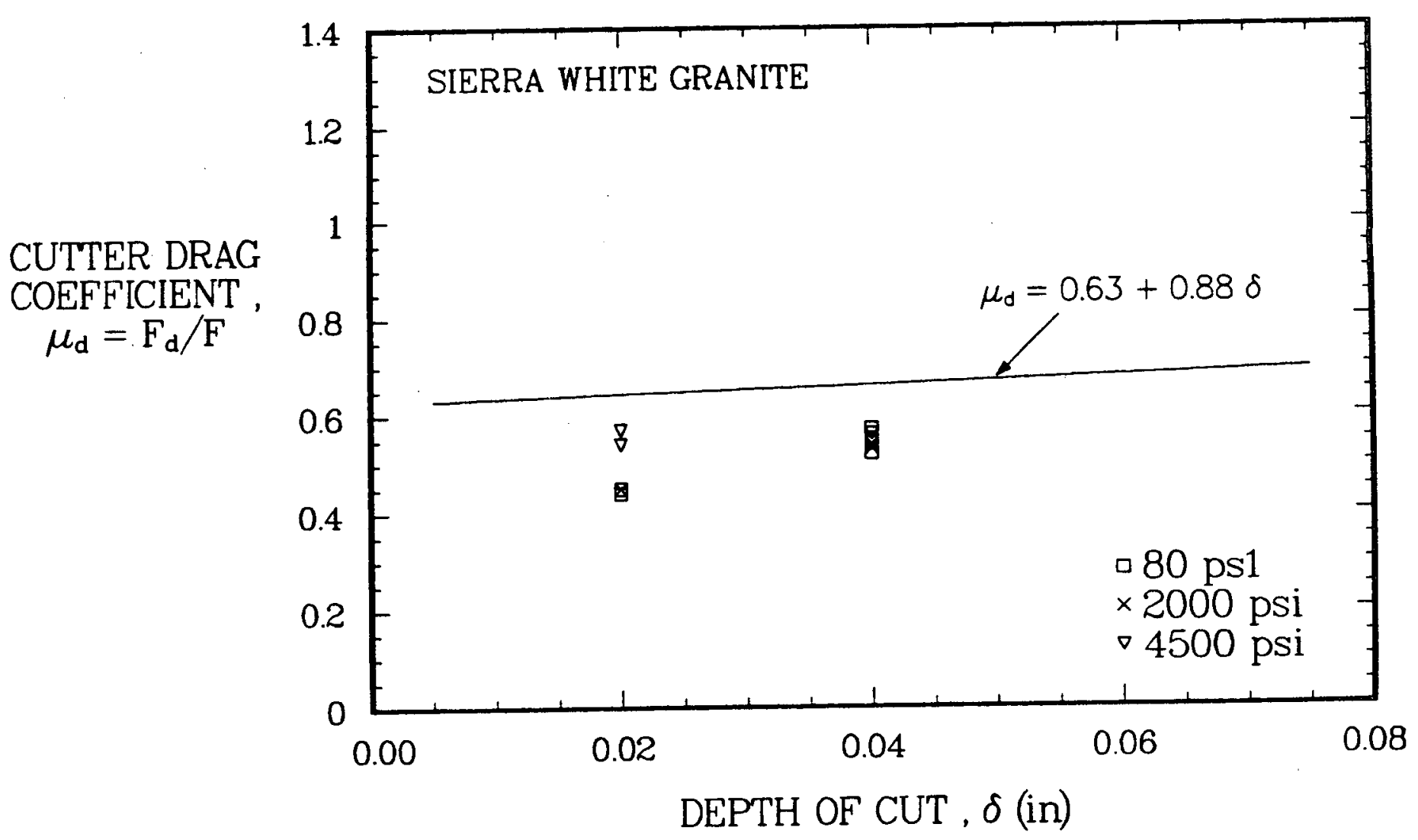

Figure 17 - Measured drag coefficients in non-interacting cuts made with
waterjet assistance. Curve represents mean data from dry cuts (Fig. 13). 


\subsection{Test Results: Interacting Cuts}

All interacting cuts were done dry. The data are tabulated in Tables A-6 and A-7 (Appendix A). These data are summarized in Figure 18. Shown here are the measured penetrating stresses as a function of the lateral distance to adjacent, pre-existing cuts. For comparison, the upper and lower dashed lines on the figures represent the penetrating stresses obtained with the dry non-interacting cuts at depths of 0.080 inch and 0.040 inch, respectively.

At large lateral distances to adjacent cuts, the geometry of the center test cut approaches that of a single, non-interacting cut of 0.080 inch depth, as seen in Figure 19. As the pre-existing adjacent cuts are placed closer together, cutter interaction reaches the level where the crosssectional area of rock removed by the center test cut is the same as that removed in a single, non-interacting cut of 0.040 inch depth. We should, therefore, expect the penetrating stress data of Figure 18 to approach the lower dashed line at small values of $d$ for each rock type and to approach the upper dashed line for each rock type at large values of $d$, as they do.

The drag coefficients measured in the interacting cut tests are tabulated in Tables A-4 and A-5 (Appendix A). These results are comparable to those obtained in the non-interacting cut tests. This indicates that the cutter drag coefficient is independent of cutter interaction. In other words, although both the penetrating force and the drag force are strongly affected by cutter interaction, the ratio of the two forces is relatively unaffected.

\subsection{Analysis of Cutter Interaction Effects}

A typical cutting pattern for a cutter on the leading face of a PDC bit is shown in Figure 20. This cutting pattern imposes a given penetrating and drag force on the cutter, depending on the type of rock, the wearflat area, and the cutting conditions. It has been shown that the ratio of drag to penetrating force in a given rock type is not heavily dependent on the depth of cut or degree of cutter interaction. There should then exist an equivalent non-interacting cut that would impose the same forces on the cutter as the actual cutting profile seen in Figure 20 . The profile of the flat rock surface in such an equivalent cut is shown as the heavy dashed line in the figure. The location of the equivalent surface relative to the bottom of the cut is $\delta$, which is defined as the effective depth of the equivalent non-interacting cut. By definition, the penetrating stress of the equivalent cut is equal to the penetrating stress of the actual cut. Since the geometry of the equivalent cut is the same as that of a noninteracting cut, the effective depth of cut is then related to the actual penetrating stress, as in Eq. 3:

$$
F / A_{W}=C_{1}\left(\delta_{e}\right)^{n 1}
$$

or, in the case of a sharp cutter, to the penetrating force, as in Eq. 4:

$$
F=c_{2}\left(\delta_{e}\right)^{n 2} .
$$




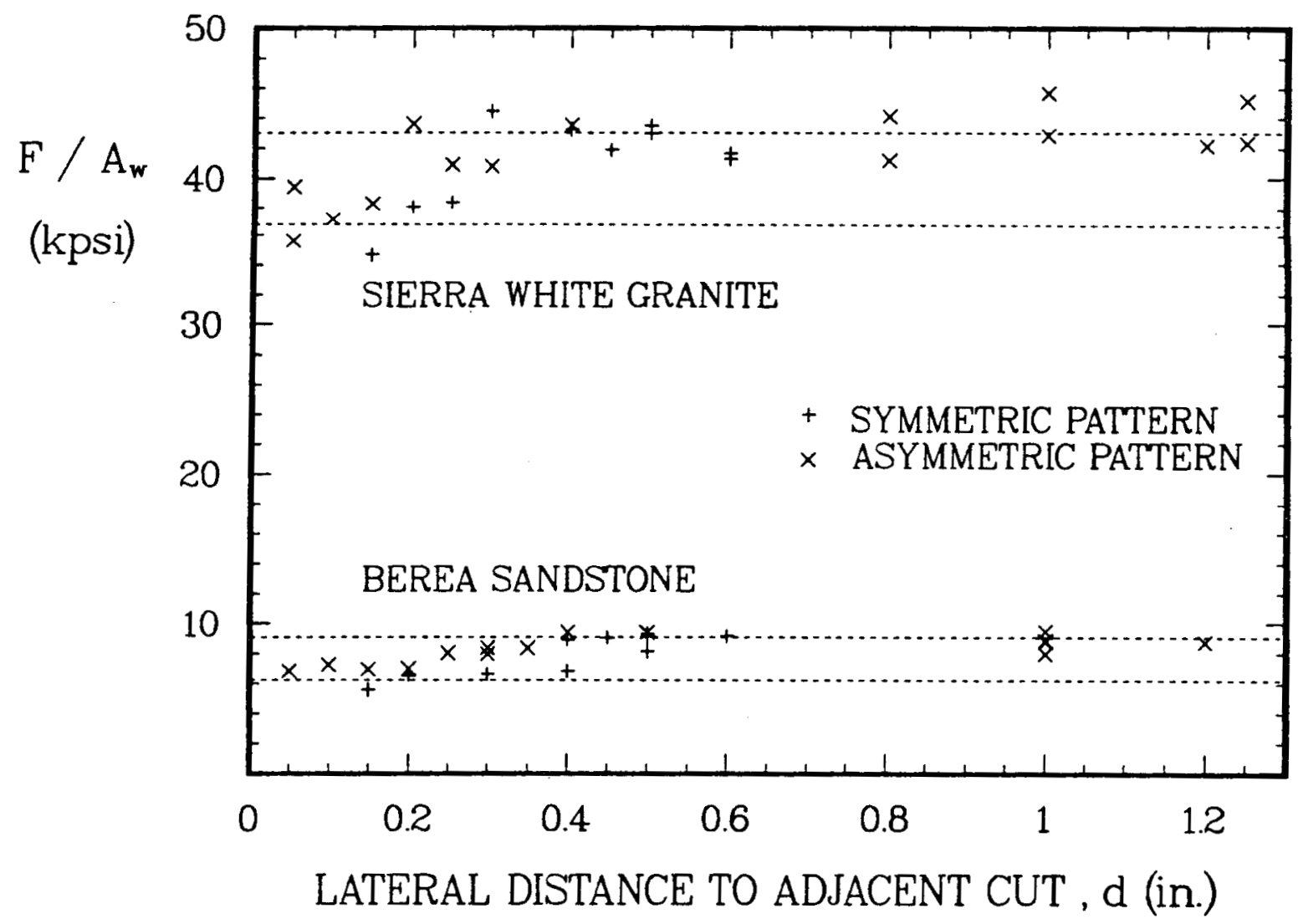

Figure 18 - Measured penetrating stresses in dxy, interacting cuts. Upper dashed line for each rock type represents mean penetrating stresses measured at 0.080 inch depth of cut in dry, noninteracting cuts. Lower dashed line for each rock type represents values measured at 0.040 inch depth of cut in dry, non-interacting cuts. 


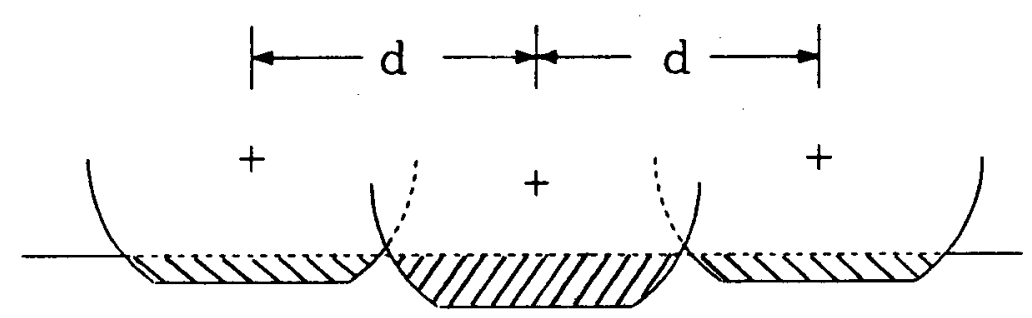

LARGE LATERAL SPACING

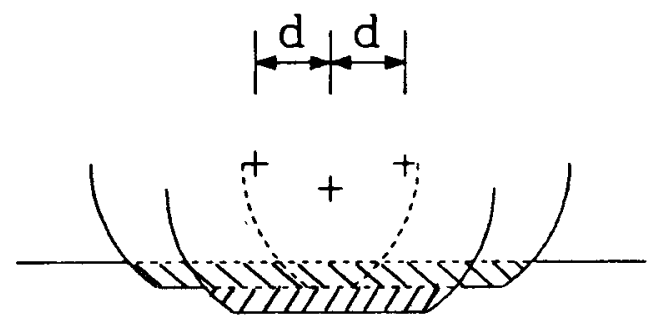

SMALL LATERAL SPACING

Figure 19 - Interacting cut patterns with large and small lateral spacing between cuts. Center cut is test cut in both cases. 


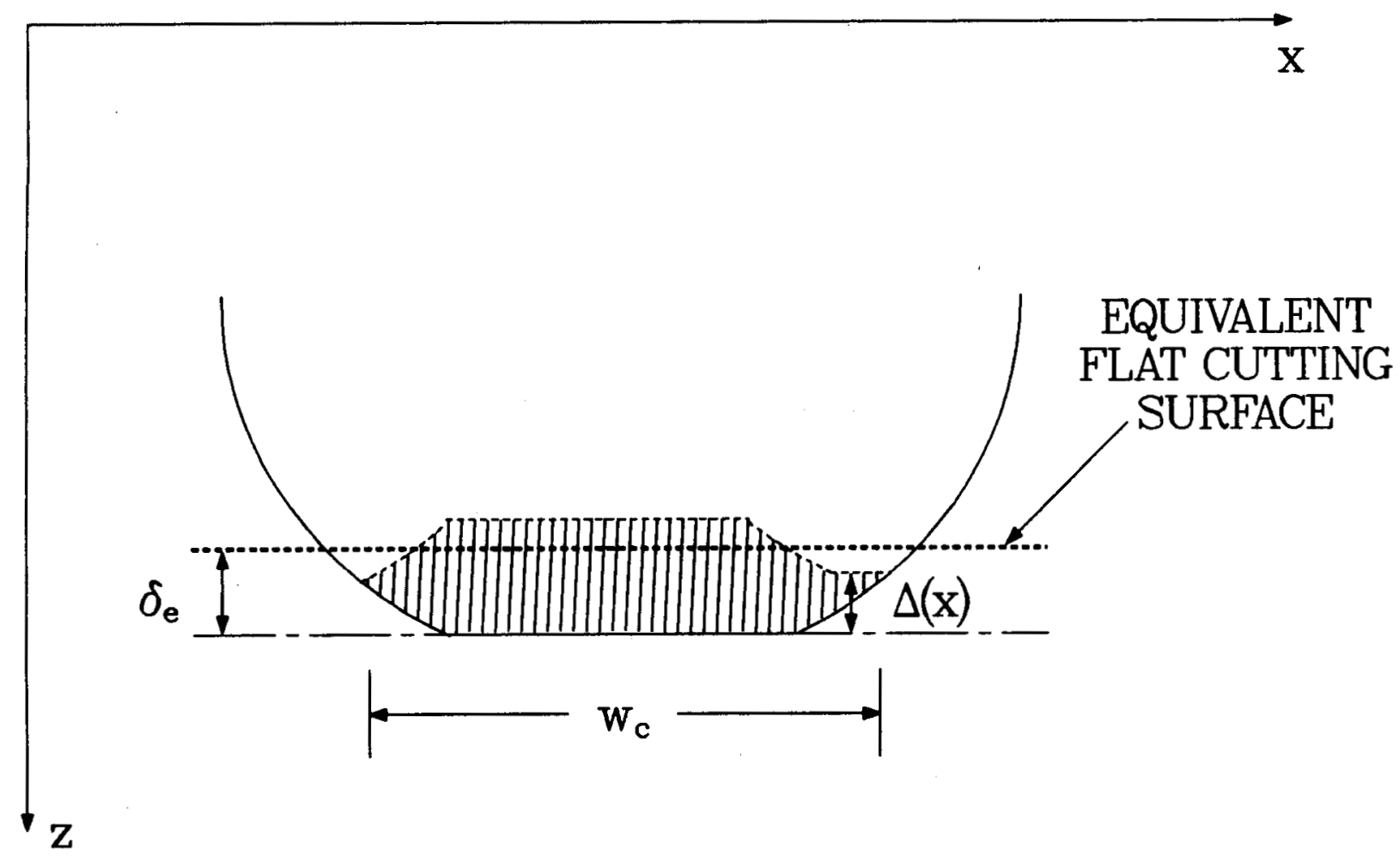

Figure 20 - Typical cutting pattern for a worn cutter on the leading face of a PDC bit. Shaded area is the actual cross-sectional area of cut. 
Inspection of Figure 20 shows that the location of the equivalent flat surface would probably be at a midpoint location between the high and low points on the actual rock cutting profile, as shown. A simple estimate of $\delta_{e}$ would then be the mean height of the actual profile:

$$
\delta_{e} \cong \frac{1}{w_{c}} \int \Delta(x) d x
$$

A computer program Eq. 9 numerically for the Figure 9 . The result asymmetric interaction patterns are shown in Figure 21. Note that for these tests, $\delta$ tends toward 0.040 inch at a small lateral distance to an adjacent cut; at a large lateral distance to an adjacent cut, $\delta_{e}$ approaches 0.080 inch.

The measured penetrating stresses associated with the computed effective depths of cut for the interacting cut tests are shown as the data points in Figure 22. The curves in these figures represent the predicted penetrating stresses given by Eq. 8a for the interacting test cuts, based on the non-interacting cut test data (Figure 10) and the described algorithm for estimating $\delta$. The good agreement between the predicted and measured penetrating forces provides the basis for developing a computer code that uses this procedure for estimating $\delta_{\mathrm{e}}$ and, subsequently, cutter forces in arbitrary bit designs.

\subsection{Other Observations}

Considerable scatter exists in the data of Figures 10-22. Since these tests were conducted under carefully controlled and monitored conditions, it is reasonable to assume that the scatter is inherent to the rock cutting process itself. For this reason, tests were generally conducted at least five times at each depth of cut and degree of cutter interaction. This ensured that the average effects of these parameters could be determined.

With the field-worn wearflats, the diamond and part of the WC-Co portion of the wearflat wore parallel to the cutting direction, as shown in Figure 23. The majority of the WC-Co portions of the wearflats, however, were generally inclined at angles of $5-10^{\circ}$ with respect to the cutting direction. This angle is defined as the circumferential wear angle. These cutters were field-worn in relatively soft formations. The relative wear resistance of PDC and WC-Co apparently dominated the wear process. The WCCo wore at a higher rate, thereby tending to keep only a small length, L, of the total wearflat length, $\mathrm{L}_{t}$, in contact with the rock. This tends to maintain the cutter in a sharper condition. The WC-Co wear along the angled surface is attributable mostly to contact with ground rock particles passing beneath this surface.

The wearflats worn in the laboratory against Sierra White granite, on the other hand, wore nearly parallel to the cutting direction (within $1^{\circ}$ ). 


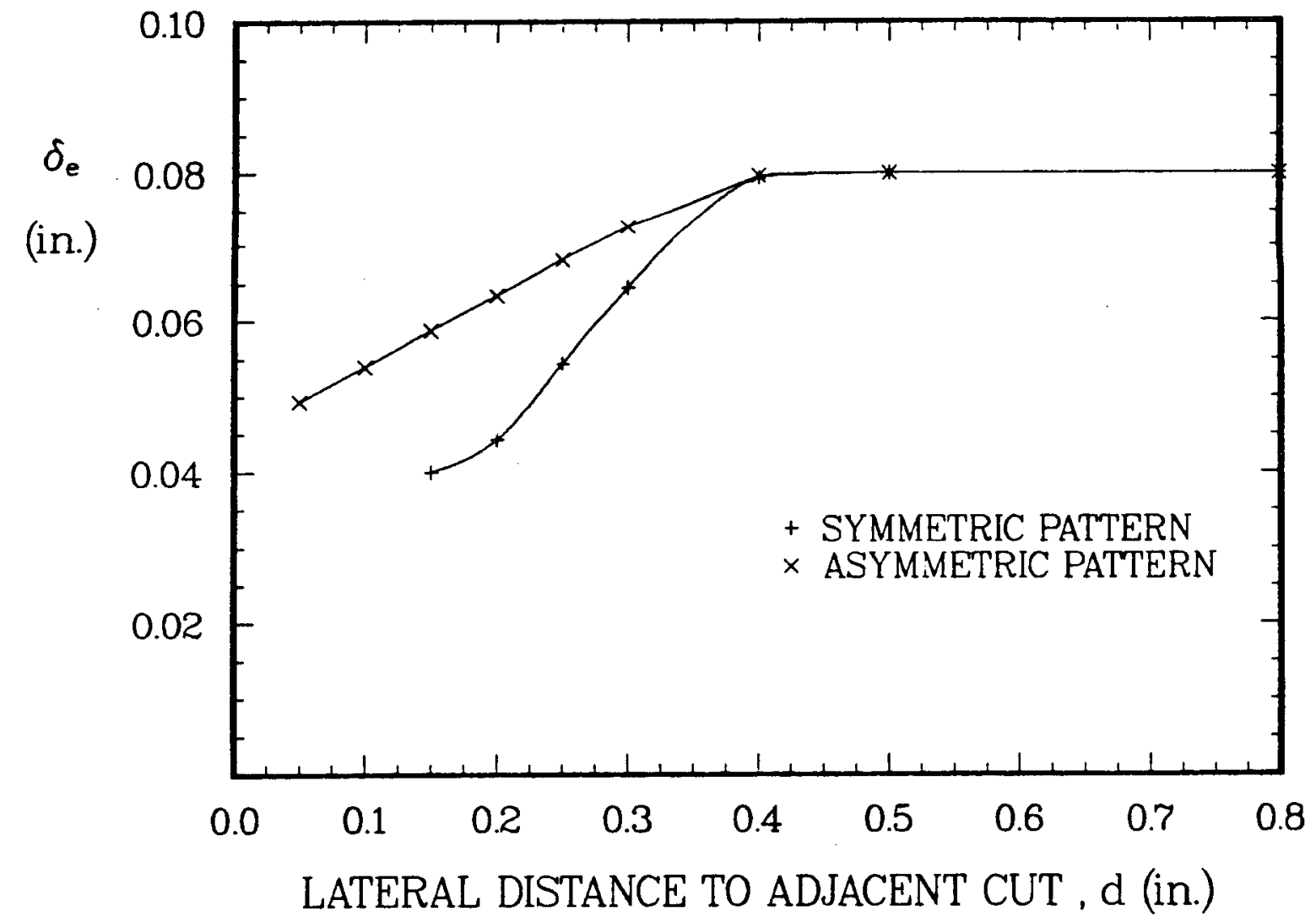

Figure 21 - Computed effective depth of cut as a function of lateral distance to adjacent cuts in interacting cut tests. 


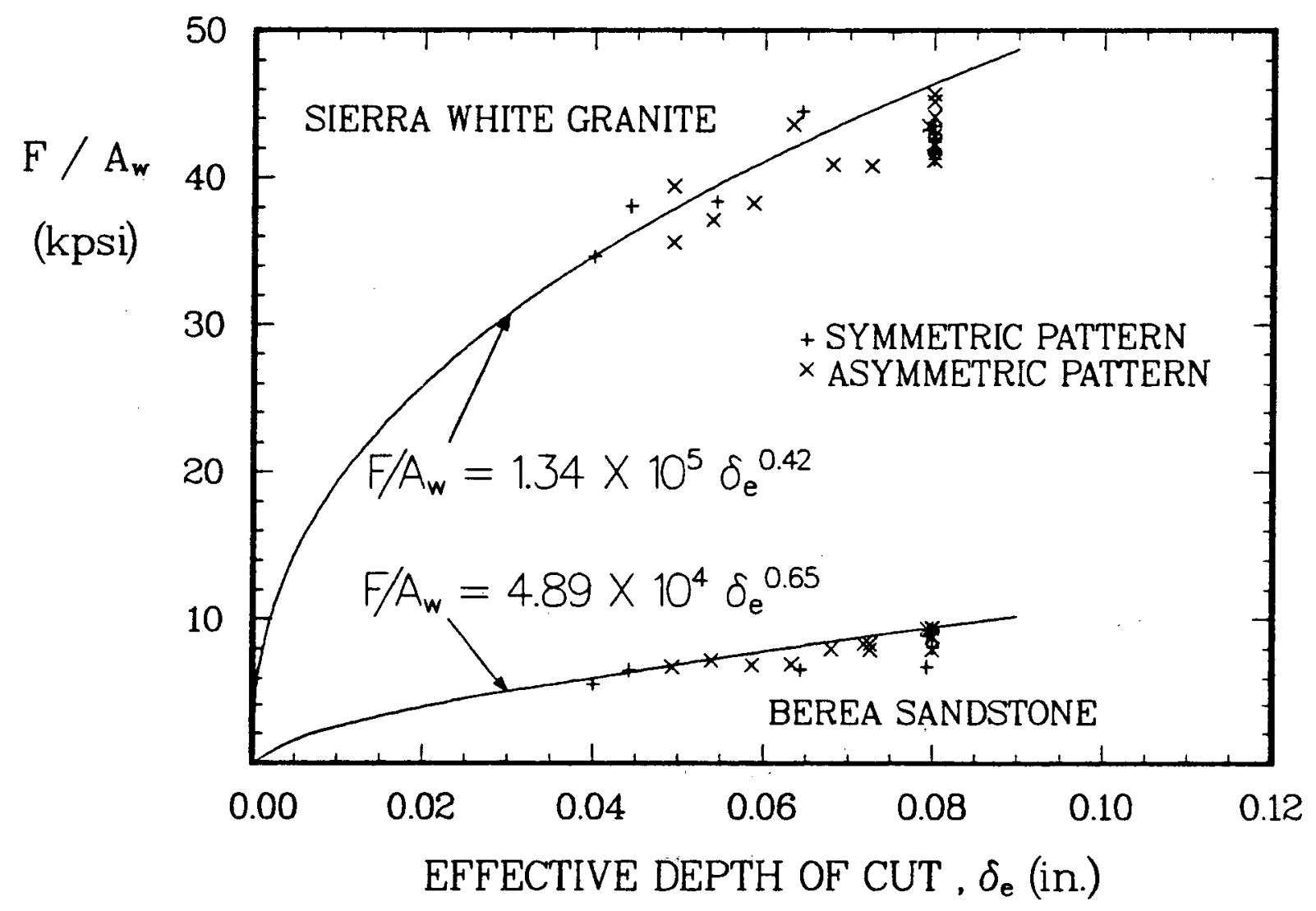

Figure 22 - Predicted and measured penetrating stresses for dry, interacting cut tests. 


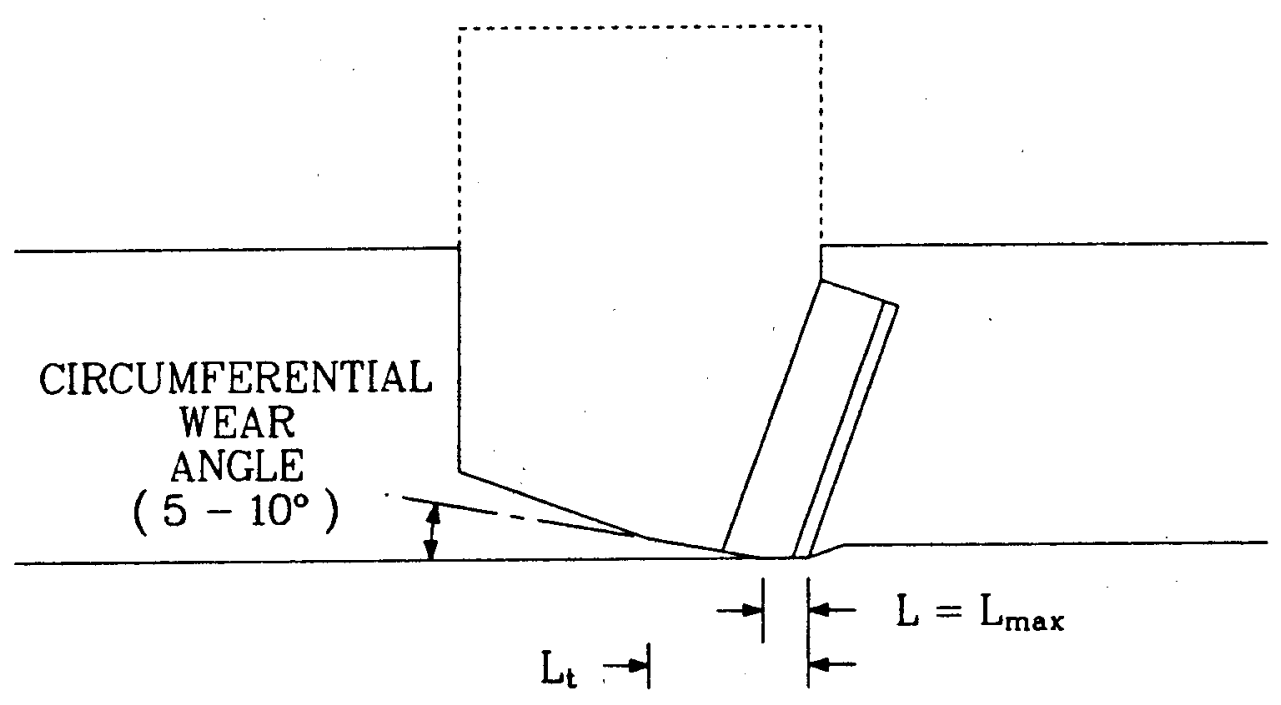

Figure 23 - Circumferential wear angle found to develop on field worn cutters. 
Field-worn cutters worn further in the laboratory against granite exhibited the same behavior. This indicates perhaps that the greater impact loading associated with hard-rock drilling, particularly in quartz-rich rock, tends to fracture off any PDC layers that are not fully supported by the WC-Co backup material. This tends to keep more of the WC-Co in contact with the rock, creating a duller cutter condition. This wear mechanism could be a major contributor to poor bit life in hard or fractured rock. 

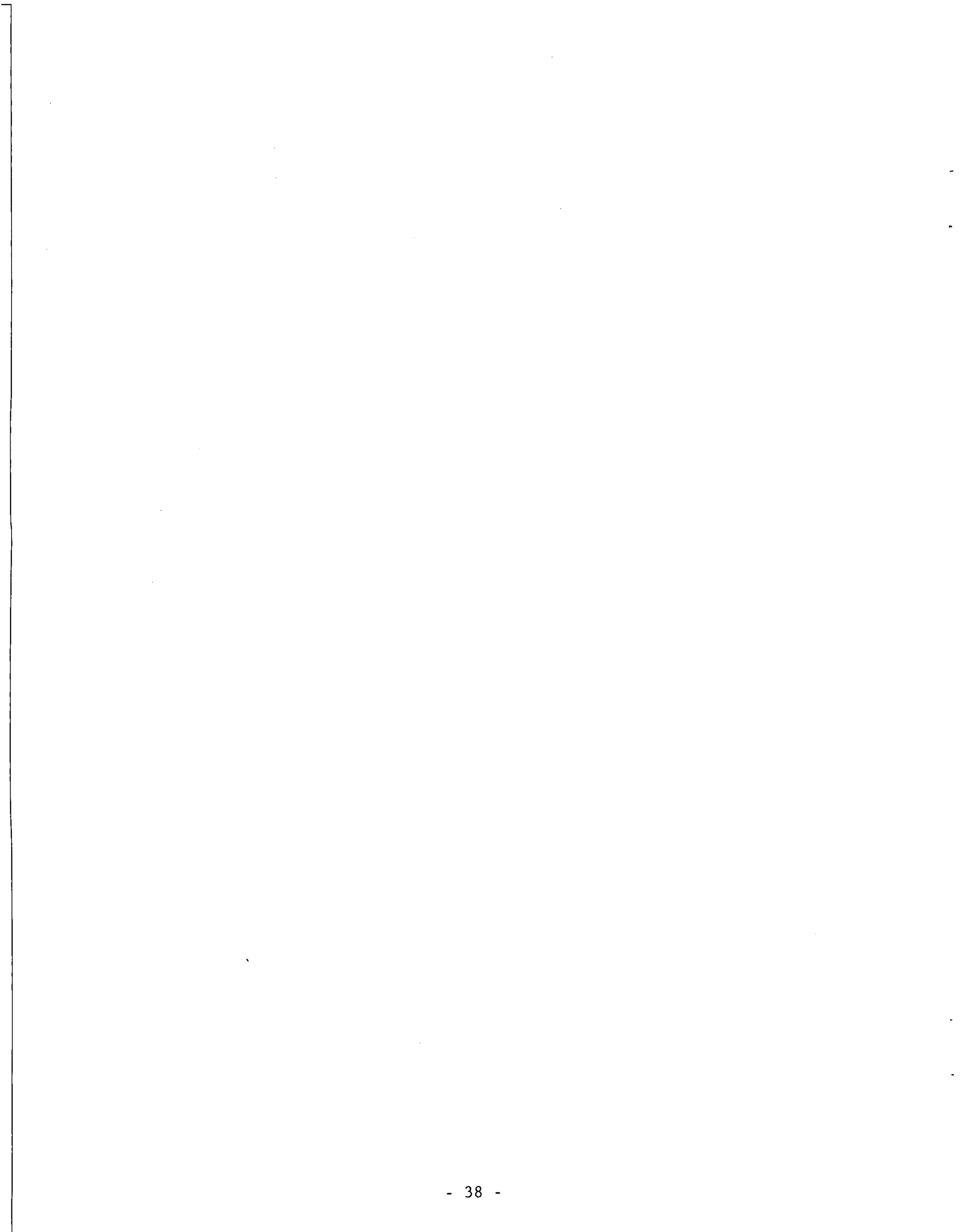

.

. 


\subsection{DEVELOPMENT OF THE COMPUTER CODE, PDCWEAR}

This section describes development of a computer code that uses the single-cutter test results as a basis for predicting bit performance. The code also uses PDC cutter wear models developed in our earlier work [31] to predict the wear of the bit and the effects of that wear on subsequent bit performance. Accordingly, the code is named PDCWEAR.

The source code for PDCWEAR is listed in Appendix B. PDCWEAR is written in FORTRAN 77, which complies with the American National Standards Institute (ANSI) standard. It was developed on a VAX 8650 computer.

The origin of the algorithm used in PDCWEAR for cutter geometry calculations is a previous computer code published by Sandia in 1982 [48]. That code, STRATAPAX, computes cross-sectional areas and volumes of cut for each cutter in an arbitrary bit design. STRATAPAX has an optimization routine that adjusts cutter radial placement in order to equalize either cutting volumes or cross-sectional areas, as specified by the user. Extensive modifications to the algorithm have been made in developing PDCWEAR code to account for the effects of wear and to calculate quantities needed to compute cutter forces, temperatures, and wear rates.

\subsection{Cutter Interaction Theory}

The geometry of a single cutter and cut profile are shown in Figure 24. The cutter inclination angle, $\phi_{c}$, defines the tilt of the cutter longitudinal axis with respect to the longitudinal axis of the bit. The radial wear angle, $\phi$, defines the location and radial inclination of the wearflat on the cutter profile. It also defines the direction of the cutter penetrating force, because the penetrating force acts normal to the wearflat.

Four coordinate systems are used to describe the cut and cutter profiles, as shown in Figure 25. The hole coordinate system is a nonrotating cartesian coordinate system whose $z$-axis is parallel to the longitudinal axis of the hole. The bit coordinate system is a cartesian coordinate system that rotates and advances with the bit and has a $z^{\prime}$-axis parallel to the longitudinal axis of the bit and parallel to the $z$-axis of the hole coordinate system. The cutter coordinate system is a twodimensional, rectangular coordinate system that lies in the plane of the diamond face of the cutter. The cutter profile coordinate system is the projection of the cutter coordinate system onto a radial plane running through the longitudinal axis of the bit.

A point $\left(x_{0}^{\prime}, z_{0}^{\prime}\right)$ in the cutter coordinate system is projected into the cutter profile coordinate system $\left(x_{0}, z_{0}\right)$ as

$$
\begin{gathered}
x_{0}=x_{0}^{\prime} \\
z_{0}=z_{0}^{\prime} \cos B,
\end{gathered}
$$




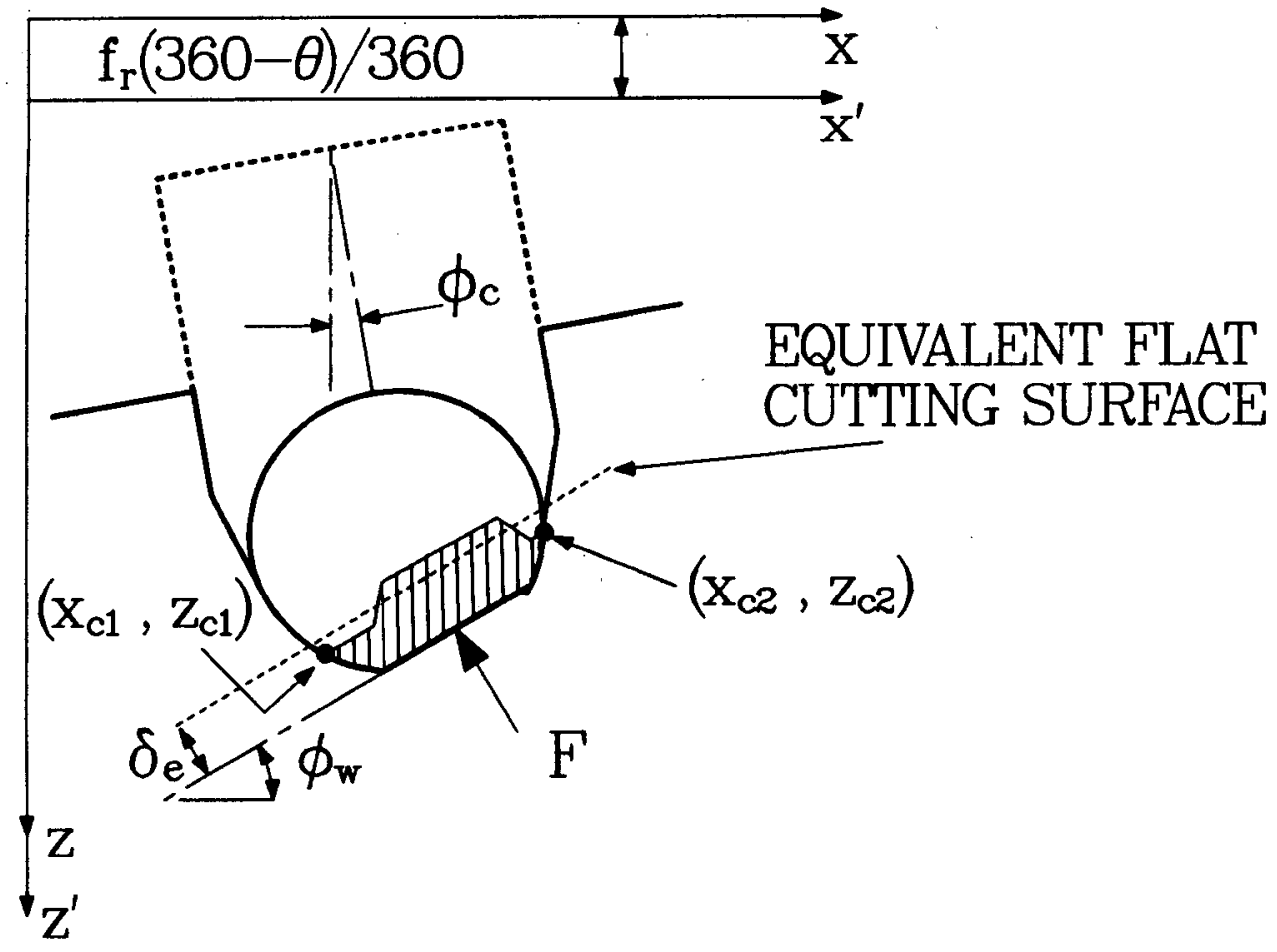

Figure 24 - Cut and cutter profiles for a general PDC cutter mounted on a bit. ( $x, y, z$ coordinate system is stationary; $x^{\prime}, y^{\prime}, z^{\prime}$ coordinate system travels with the bit.) 


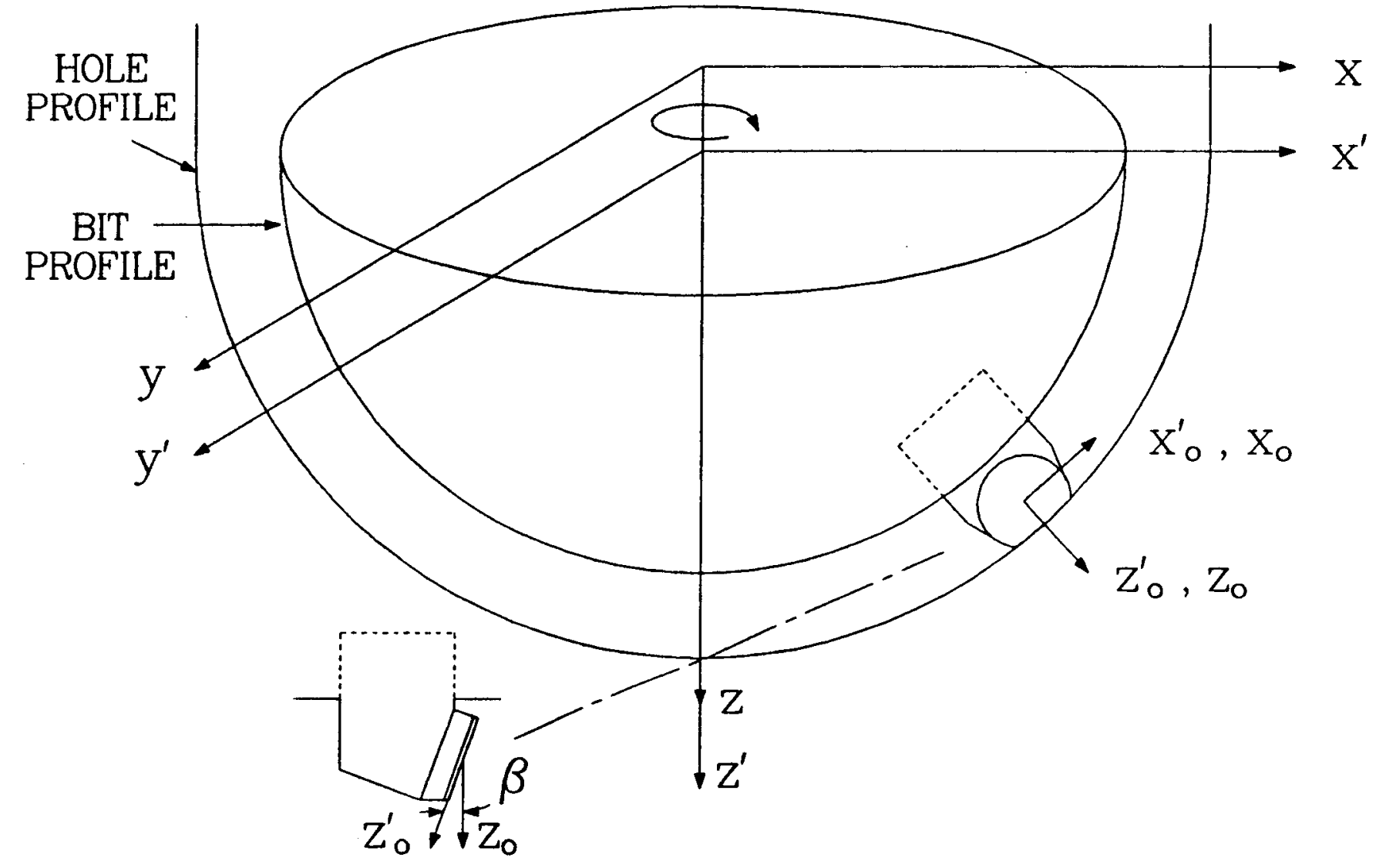

Figure 25 - Schematic of the four coordinate systems used to describe cut and cutter profiles. Hole $(x, y, z)$, bit $\left(x^{\prime}, y^{\prime}, z^{\prime}\right)$, cutter $\left(x_{0}^{\prime}, z_{0}^{\prime}\right)$, and cutter profile $\left(x_{0}, z_{0}\right)$ coordinate systems. 
where $\beta$ is the cutter backrake angle. The circular edge of a sharp cutter in the cutter coordinate system is described by the equation:

$$
\left(\frac{x_{0}^{\prime}}{r}\right)^{2}+\left(\frac{z_{0}^{\prime}}{r}\right)^{2}=1 \text {. }
$$

where $r$ is the radius of the circular cutter compact. Transforming this equation to the cutter profile coordinate system, the cutter profile proves to be one of an ellipse:

$$
\left(\frac{x_{0}}{r}\right)^{2}+\left(\frac{z_{0}}{b}\right)^{2}=1 \text {. }
$$

where

$$
\mathrm{b}=\mathrm{r} \cos \mathrm{B} .
$$

During one revolution of the bit, each cutter passes through the $x-z$ plane of the hole coordinate system. As each cutter passes through this plane, the transformation equations between the hole coordinate system $(x, y, z)$ and that cutter's profile coordinate system $\left(x_{0}, z_{0}\right)$ are (see Figure 26):

$$
x=R+x_{0} \cos \phi_{c}+z_{0} \sin \phi_{c}
$$

and

$$
\mathbf{y}=0,
$$

$$
z=H-x_{0} \sin \phi_{c}+z_{0} \cos \phi_{c},
$$

where

$$
H=H^{\prime}+f_{r}(360-\theta) / 360 \text {. }
$$

Here $R$ and $H^{\prime}$ are the radial and longitudinal locations, respectively, of the center of the cutter compact on the bit body. The quantity $\mathrm{H}$, defined as the cutting height of the cutter, accounts for the fact that the bit advances in the $z$-direction as it rotates. This height, which is the $z$ coordinate of the center of each cutter as it passes through the $x-z$ plane of the hole coordinate system is a function of the feed per revolution, $f$, and the angular position, $\theta$, of the cutter on the bit face. The angular position (in degrees) is defined as positive in the counterclockwise direction (looking at the face of the bit). The feed rate is related to the rate of penetration, $\mathrm{ROP}$, and the bit rotary speed, $\mathrm{N}$ :

$$
\mathbf{f}_{\mathbf{r}}=\mathrm{ROP} / \mathrm{N} \text {. }
$$



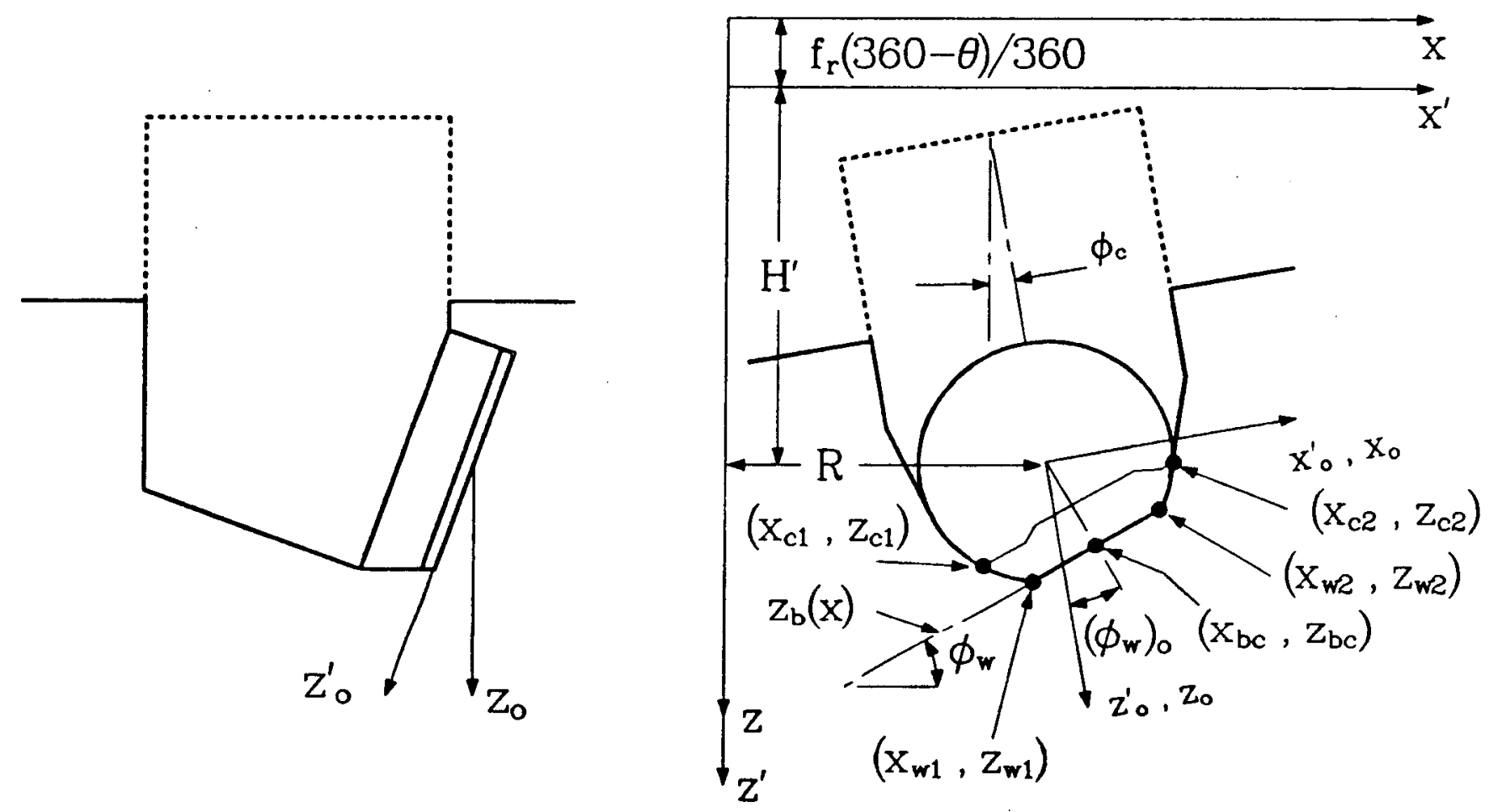

Figure 26 - Detailed definition of several points used in cutter geometry analysis. 
Combining Eqs. 12 and 13 we obtain the cutting profile $(x, y, z)$ of a sharp cutter in terms of the hole coordinate system:

$$
\begin{gathered}
z=C_{3}\left[D(x-R) \pm \sqrt{E-(x-R)^{2}}\right]+H, \\
y=0,
\end{gathered}
$$

where

$$
\begin{gathered}
\mathrm{C}_{3}=\frac{r b}{E} \\
D=-\sin \phi_{c} \cos \phi_{c}\left(r^{2}-b^{2}\right) / r b \\
E=r^{2} \cos ^{2} \phi_{c}+b^{2} \sin ^{2} \phi_{c} .
\end{gathered}
$$

At any point on the $\mathrm{x}$-axis, these equations define the $\mathrm{z}$-coordinate of the cutting edge of each sharp cutter as it passes through the vertical plane.

Wear modifies the cutting profile according to the wearflat width, w, and the radial wear angle, $\phi_{w}$. An algorithm in the program iterates upon the wear angle by initially assuming $\phi_{w}=\phi_{c}$. The wearflat geometry of each cutter is then computed based on this initial guess. The cut profile for each cutter is determined using the algorithm described below. New estimates of $\phi_{\mathrm{w}}$ are then made for each cutter by assuming that the wearflat of a cutter develops parallel to the overall radial slope of the rock surface encountered by that cutter, as shown in Figure 24 . In other words,

$$
\phi_{w}=\tan ^{-1}\left[\frac{z_{c 1}-z_{c 2}}{x_{c 2}-x_{c 1}}\right] \text {. }
$$

Since the new estimate of $\phi_{\mathrm{w}}$ may be different from the initial guess, the wearflat locations on the cutters may change from the previous iteration, which in turn changes the cut profiles. The process is repeated until the solution converges for all cutters.

As suggested by Eq. 15, the location of the wearflat on the cutting profile is a function of the interaction experienced by that cutter. The wearflat modifies the cutter profile over the range

$$
\mathbf{x}_{\mathrm{w} 1}<\mathrm{x}<\mathrm{x}_{\mathrm{W} 2}
$$

seen in Figure 26, and for values of $x$ in this range, Eq. 14 is not valid. Instead, the cutter profile within this range is described by the line, 


$$
z=z_{w 1}+\left[\frac{x-x_{w 1}}{x_{w 2}-x_{w 1}}\right]\left(z_{w 2}-z_{w 1}\right)
$$

We must, therefore, derive expressions for $\left(\mathrm{x}_{\mathrm{w} 1}, \mathrm{z}_{\mathrm{w} 1}\right)$ and $\left(\mathrm{x}_{\mathrm{w} 2}, \mathrm{z}_{\mathrm{w} 2}\right)$.

The cutter wearflat is easiest described in the cutter coordinate system. In this system, the wear angle $\left(\phi_{\mathrm{w}}\right)_{0}^{\prime}$ is related to the wear angle in the hole coordinate system with the equation

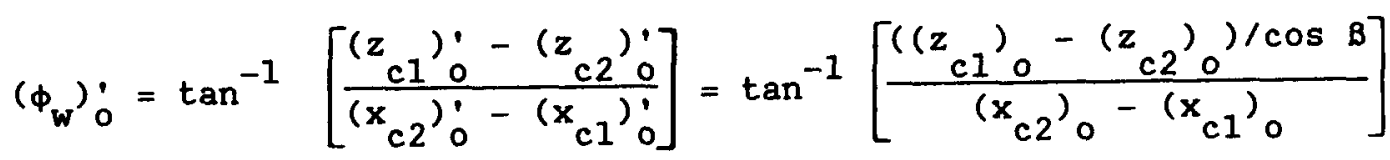

or

$$
\left(\phi_{W}\right)=\tan ^{-1}\left[\tan \left(\phi_{W}\right)_{0} / \cos B\right],
$$

where

$$
\left(\phi_{w}\right)_{0}=\phi_{w}-\phi_{c}
$$

The wearflat width, $w$, is measured in the cutter coordinate system. In the circular geometry of this system, geometry considerations give the results,

$$
\begin{aligned}
& \left(x_{w 1}\right)_{0}^{\prime}=r_{w} \sin \left(\phi_{w}\right) \dot{0}-\frac{w}{2} \cos \left(\phi_{w}\right): \\
& \left(z_{w 1}\right) \dot{0}=r_{w} \cos \left(\phi_{w}\right) \dot{0}+\frac{w}{2} \sin \left(\phi_{w}\right) \dot{0} \\
& \left(x_{w 2}\right)_{0}^{\prime}=r_{w} \sin \left(\phi_{w}\right) !+\frac{w}{2} \cos \left(\phi_{w}\right) ! \\
& \left(z_{w 2}\right)_{0}^{j}=r_{w} \cos \left(\phi_{w}\right)_{0}^{\prime}-\frac{w}{2} \sin \left(\phi_{w}\right)_{0}
\end{aligned}
$$

where

$$
r_{w}=\sqrt{r^{2}-\left(\frac{w}{2}\right)^{2}}
$$

The profile of a worn cutter can now be determined by using Eqs. 14-19.

The computer algorithm that implements the above equations is illustrated in Figure 27. The $x$-axis is divided into a number of equalsized elements of length $\Delta x$. At each value of $x$ corresponding to the midpoint of these elements, a procedure determines which cutters interact at that cutting radius. For each of these cutters, the program tests whether

$$
\mathbf{x}_{\mathrm{w} 1}<\mathrm{x}<\mathbf{x}_{\mathrm{w} 2}
$$




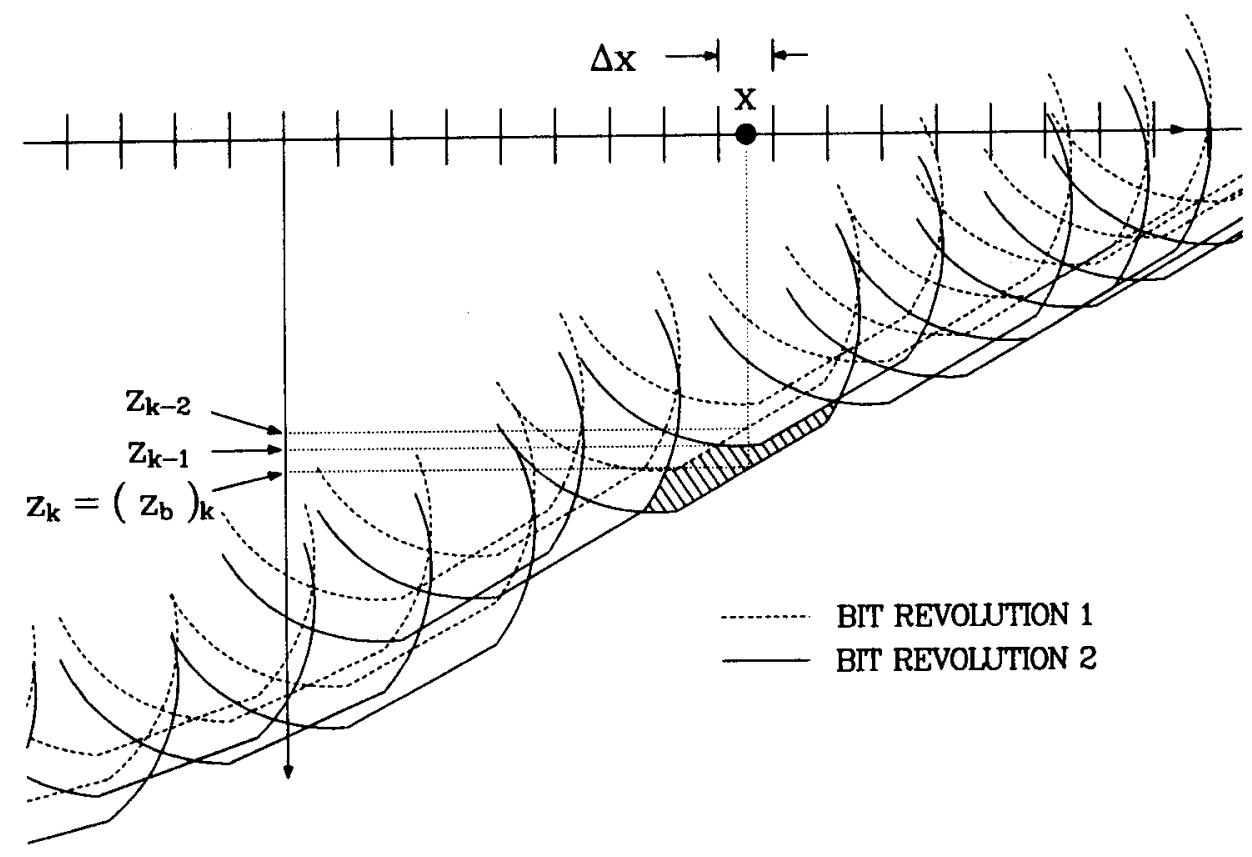

Figure 27 - Schematic of algorithm used in PDCWEAR to compute $z$-coordinates of cutting profiles at each value of $x$. 
If $x$ falls within this range, Eq. 17 is used to determine $z_{k}$, the $z-$ coordinate of the $k$ th cutter profile encountered at that radial position $x$. If $x$ is not in this range for a given interacting cutter, Eq. 14 is used to determine $z_{k}$. In either case, the incremental cross-sectional area of cut,

$$
\Delta A_{r}=\Delta x\left(z_{k}-z_{k-1}\right)
$$

is assigned to the kth cutter. The incremental volume of cut,

$$
\Delta V_{r}=2 \pi \times\left(\Delta A_{r}\right)
$$

is assigned to the same cutter. This procedure is repeated for the $k-1$ cutter encountered at $x$, then the $k-2$ cutter, etc.

By definition, the equivalent cutting surface in the cutter interaction model is inclined at the radial wear angle, $\phi_{w}$. Since the penetrating force is normal to the wearflat, this implies ${ }^{\mathrm{w}}$ that the penetrating force is directed at the angle $\phi_{\mathrm{w}}$ with respect to the longitudinal axis of the bit. Depending on cutter Interaction, $\phi$ may not equal $\phi$, and the penetrating force may not develop parallel to the longitudinal axis of the cutter, resulting in a cutter side force.

The effective depth of cut must be computed in the same direction as the penetrating force vector. The equivalent bottom of each cut is described by the line (see Fig. 26):

$$
z_{b}=z_{b c}+\left(x_{b c}-x\right) \tan \phi_{w}
$$

where

$$
\begin{gathered}
z_{b c}=H-\left(x_{b c}\right)_{0} \sin \phi_{c}+\left(z_{b c}\right)_{0} \cos \phi_{c} \\
x_{b c}=R+\left(x_{b c}\right)_{0} \cos \phi_{c}+\left(z_{b c}\right)_{0} \sin \phi_{c} \\
\left(z_{b c}\right)_{0}=\left[r_{w} \cos \left(\phi_{w}\right)_{0}^{\prime}\right] \cos B \\
\left(x_{b c}\right)_{0}=r_{w} \sin \left(\phi_{w}\right)_{o}^{\prime}
\end{gathered}
$$

and $\left(\phi_{W}\right)^{\prime}$ and $r_{y}$ are given by Eqs. 18 and $19 \mathrm{e}$, respectively. At each point $x$, the ${ }^{\circ} z$-location of the bottom of the kth cut, $\left(z_{b}\right)_{k}$, is given by Eq. 23 . The effective depth of cut for each cutter is then

$$
\delta_{e}=\frac{1}{n_{x}} \sum_{i=1}^{n_{x}} \Delta_{i},
$$


where

$$
\Delta_{i}=\left[\left(z_{b}\right)_{k}-(z)_{k-1}\right] \cos \phi_{w}
$$

and $\mathrm{n}$ is the number of $\Delta \mathrm{x}$ elements over which a given cutter interacts with the rock formation.

\subsection{Forces and Moments Acting on the Bit}

Once the effective depths of cut are computed, the cutter penetratirig force can be estimated using Eq. 8b for sharp cutters and Eq. 8a for worn cutters. The algorithm used in PDCWEAR computes penetrating forces based on both equations and uses the larger of the two computed values. This logic is based on the fact that $\mathrm{Eq} .8 \mathrm{a}$ is not valid with very small wearflat areas because the crushed rock beneath the cutter wearflat tends to distribute the penetrating force over an area larger than the small wearflat area. Since the lowest penetrating force possible is the one obtained with a sharp cutter, it is evident that Eq. $8 \mathrm{~b}$ is more accurate than Eq. $8 \mathrm{a}$ in cases where Eq. $8 \mathrm{~b}$ predicts higher forces.

It should be noted that in cases where the wearflat develops on the side of the cutter (i.e., $\phi_{\mathrm{w}} \neq \phi_{\mathrm{c}}$ ), some inaccuracies in computed cutter forces will occur. This is caused by the fact that in such cases, the angular transformation converts some of the cutter backrake to a small effective siderake angle. This factor is not significant for the relatively small backrake angles generally used in PDC bits $\left(5-20^{\circ}\right)$.

As seen in Section 2.3, the cutter correlation constants $C_{1}, n 1, C_{2}$, and $\mathrm{n} 2$ can be significantly affected by waterjet assistance. The effects of other factors that control inherent drillability, such as backrake angle, are also implicitly contained within the values used for these parameters. As a result, PDCWEAR is written so that two different types of cutter can be specified, each with its own set of correlation constants. In this way, the effects of different design options, such as providing waterjet assistance to selected cutters on a bit face, can be assessed.

After the penetrating force is computed using the appropriate correlation constants, it may be resolved into radial and longitudinal (vertical) components in the bit coordinate system:

$$
F_{\mathbf{r}}=-F \sin \phi_{W}
$$

and

$$
F_{V}=-F \cos \phi_{w} .
$$

The drag force on each cutter is estimated using Eq. 5b. This force is directed opposite to the direction of the cutter velocity vector. In terms of the bit coordinate system, each drag force is a circumferential force about the longitudinal axis. 
The resultant bit forces and moments arising from the cutter forces can now be determined. The total weight on bit (WOB) is simply the sum of the longitudinal components, as depicted in Figure 28:

$$
\text { WOB }=\sum_{j=1}^{n_{c}}\left(-F_{v}\right)_{j} \text {, }
$$

where $n$ is the number of cutters on the bit. The drilling torque is the sum of the moments caused by the circumferential drag forces:

$$
T=\sum_{j=1}^{n}\left(F_{d}\right)_{j}\left(x_{b c}\right)_{j},
$$

where $\mathrm{x}_{\mathrm{bc}}$ is defined in Eq. 23.

Unless the cutters are placed such that the cutting forces balance each other, there will be a net side force acting on the bit. The component of the side force in the $\mathrm{x}^{\prime}$-direction is

$$
F_{x}^{\prime}=\sum_{j=1}^{n}\left[\left(F_{r}\right)_{j} \cos (\theta)_{j}+\left(F_{d}\right)_{j} \sin (\theta)_{j}\right] \text {. }
$$

Likewise, in the $\mathrm{y}^{\prime}$-direction,

$$
F_{y}^{\prime}=\sum_{j=1}^{n}\left[\left(F_{r}\right)_{j} \sin (\theta)_{j}-\left(F_{d}\right)_{j} \cos (\theta)_{j}\right] \text {. }
$$

giving a resultant side force of

$$
F_{s}=\sqrt{\left(F_{x}^{\prime}\right)^{2}+\left(F_{y}^{\prime}\right)^{2}}
$$

A different mode of bit imbalance is the net bending moment about the $x^{\prime}$ and $y^{\prime}$ axes:

$$
\begin{aligned}
& M_{x}^{\prime}=\sum_{j=1}^{n}\left[-\left(F_{r}\right)_{j} \sin (\theta){ }_{j}\left(z_{b c}^{\prime}\right)_{j}+\left(F_{v}\right)_{j}\left(x_{b c}\right)_{j} \sin (\theta){ }_{j}\right. \\
& \left.+\left(F_{d}\right)_{j} \cos (\theta)_{j}\left(z_{b c}^{\prime}\right)_{j}\right], \\
& M_{y}^{\prime}=\sum_{j=1}^{n}\left[\left(F_{r}\right)_{j} \cos (\theta)_{j}\left(z_{b c}^{\prime}\right)_{j}-\left(F_{v}\right)_{j}\left(x_{b c}\right)_{j} \cos (\theta){ }_{j}\right. \\
& \left.+\left(F_{d_{j}}\right)_{j} \sin (\theta)_{j}\left(z_{b c}^{\prime}\right)_{j}\right],
\end{aligned}
$$



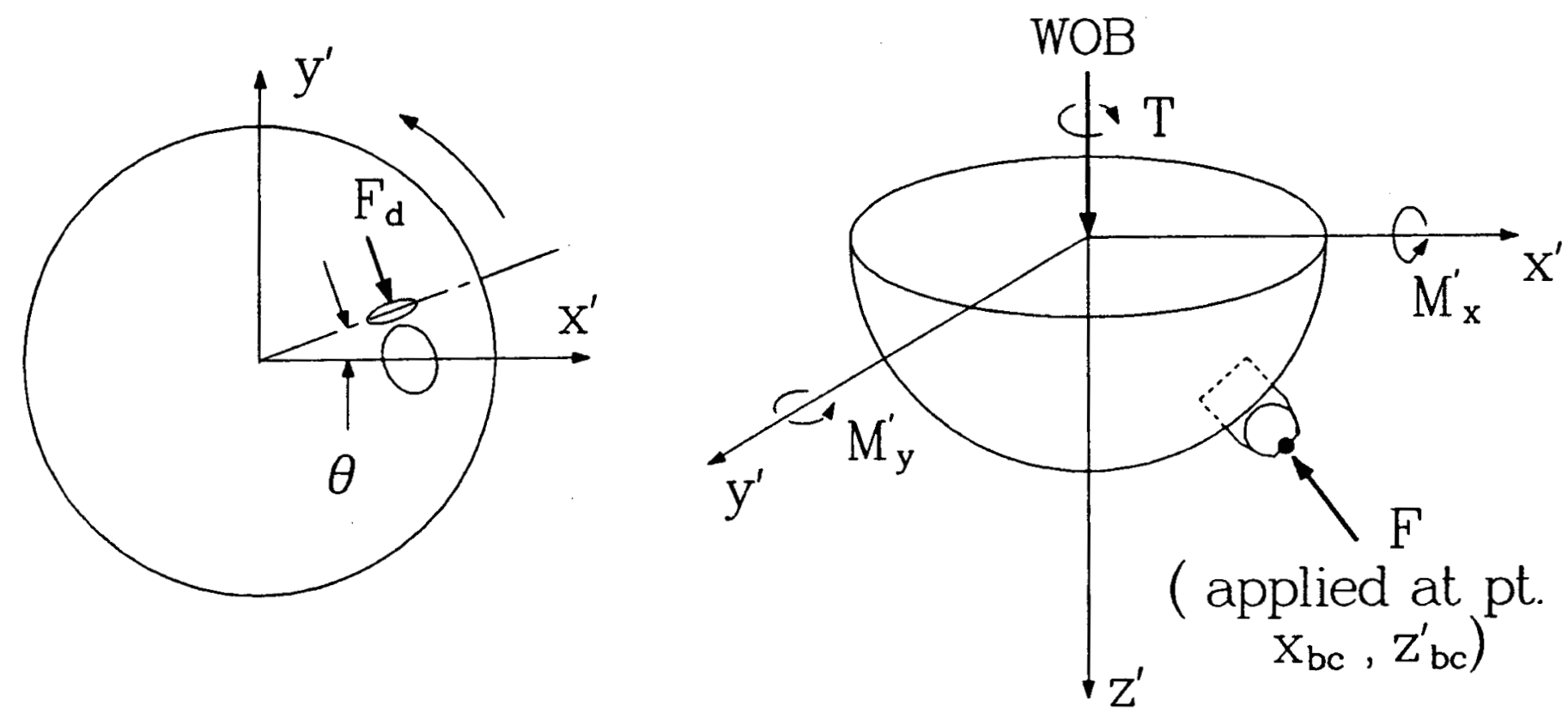

Figure 28 - Schematic showing how cutter forces are integrated to produce bit performance parameters. 
where

$$
z_{b c}^{\prime}=z_{b c}-f_{r}(360-\theta) / 360
$$

and $x_{b c}$ and $z_{b c}$ are defined in Eq. 23 .

\subsection{Cutter Wearflat Temperatures}

The cutter wearflat temperatures are calculated using an equation developed in our earlier work $[22,26,27]$ :

$$
\overline{\mathrm{T}}_{\mathrm{w}}=\mathrm{T}_{\mathrm{f}}+\frac{\mu \mathrm{FVf}}{\mathrm{A}_{\mathrm{w}}}\left[1+\frac{3 \sqrt{\pi}}{4} \mathrm{fk}_{2}\left(\frac{\mathrm{V}}{\mathrm{L} \mathrm{x}_{2}}\right)^{1 / 2}\right]^{-1},
$$

where $\mathrm{T}_{f}$ is the downhole fluid temperature; $\mu$ is the cutter/rock friction coefficient; $\quad V$ is the cutting speed; $f$ is the thermal response function; $L$ is the wearflat length in the cutting direction; $k_{2}$ is the thermal conductivity of the rock; and $\chi_{2}$ is the thermal diffusivity of the rock. The cutting speed of each cutter is dependent on the rotary speed, $N$, of the bit and the radial location of the wearflat, $x_{b c}$ :

$$
\mathrm{V}=2 \pi \mathrm{N} \mathrm{x}_{\mathrm{bc}}
$$

The thermal response function, $f$, is defined in our earlier work as the temperature rise of the cutter wearflat per unit frictional heat flowing into the cutter at the cutter/rock interface. The numerical value of $f$ depends on the geometry of the cutter, material properties of the cutter and rock, and the cutter convective cooling coefficient, h. In our earlier work [27], we used a finite element thermal model to compute values of $f$ for several different combinations of these parameters with a stud-mounted cutter employing 0.5-inch diameter PDC compacts. In the present study, we have obtained values of $f$ for 0.75 -inch diameter compacts, using the same approach.

Shown in Figure 29 is the finite element mesh used for one of the 0.75 inch cutter configurations assumed in this study. Shown here is a cutter with a severely worn compact. In addition to its larger compact size, this cutter also has a larger stud than has been traditionally used with the smaller compacts: 1.0 inch, compared with 0.625 inch, respectively. Because of the larger compact diameter, it is considered desirable to countersink the cutter to prevent the bit body-rock surface standoff distance from becoming excessive. Excessive standoff can reduce drilling fluid velocities across the face of the bit to levels below those required for proper cutter cooling and bottomhole cleaning $[23,27]$.

The results for the severely worn compact and the other wear configurations considered in this study are presented in Appendix $C$, which also describes the thermal analysis in more detail. The results indicate that for most drilling conditions of interest, larger cutters generally have 


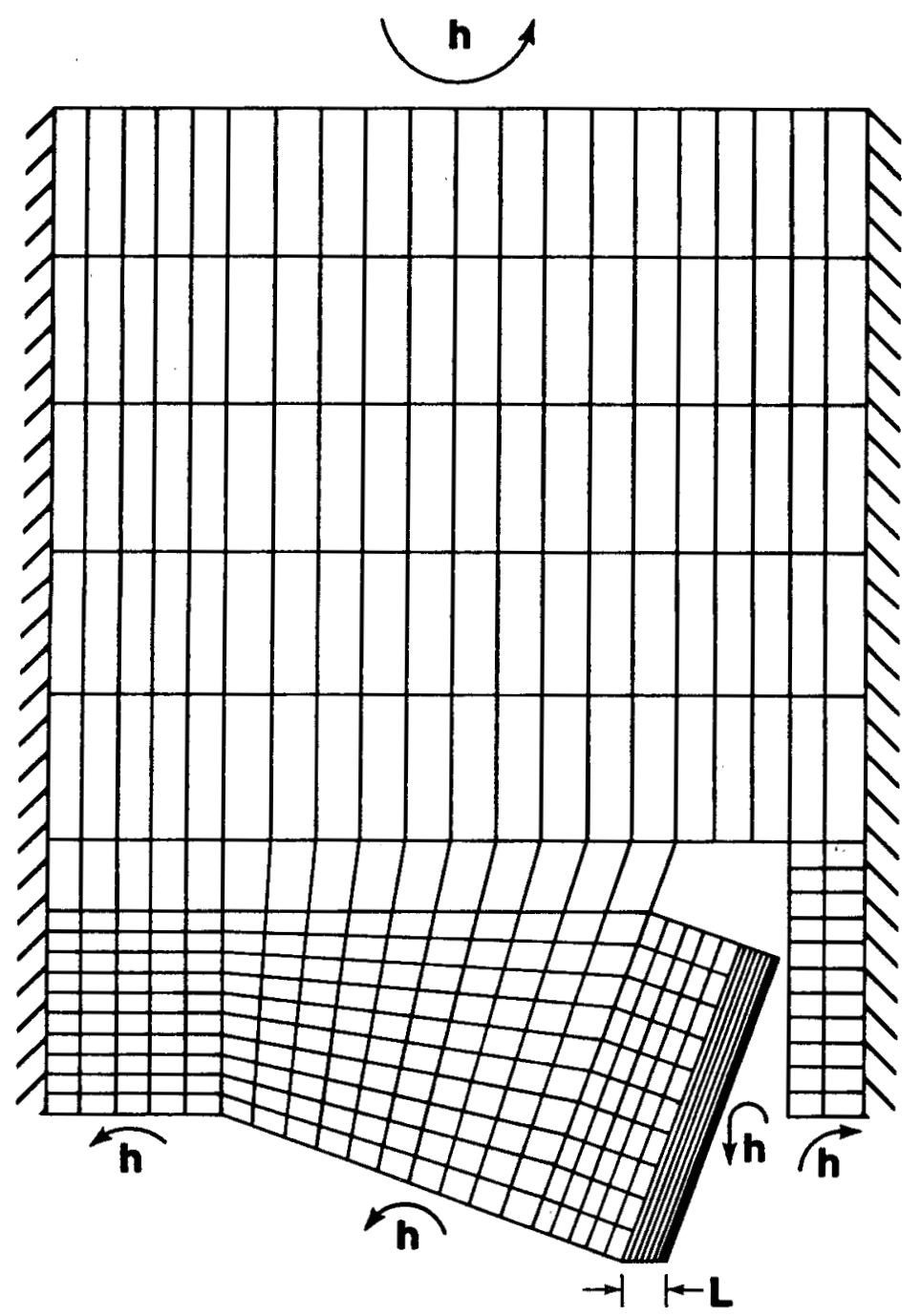

Figure 29 - Finite element mesh used to compute thermal response of 0.75 inch cutters. 
larger values of $f$ and thus run hotter than smaller cutters. This is caused by the longer mean path through the cutter for heat being conducted from the wearflat to a cooling surface. The higher temperatures, however, are relatively insignificant and are compensated for by the improved cutting efficiency inherent with larger cutters.

PDCWEAR uses an interpolation scheme to determine $f$ for any given combination of cutter compact radius, $r$, wearflat length, $L$, and convective cooling coefficient, $h$. The range in cooling coefficient for which the computed value of $f$ is backed by numerical results is 1.76 to $1.76 \times 10^{4}$ $\mathrm{Btu} / \mathrm{hr} \mathrm{ft}^{2} \mathrm{O}$. The range in cutter radius that is backed by numerical results is only 0.25 to 0.375 inch ( 0.5 - to 0.75 -inch diameter). All values of $f$ computed by the program assume stud-mounted PDC cutters with a $20^{\circ}$ backrake, conventional cutter materials, and a 0.025-inch thick diamond layer on the PDC face. Thermal effects related to parameters such as cutter material thermal conductivity, diamond layer thickness, and bit balling can be assessed for 0.5 -inch cutters by replacing the base values of $f$ tabulated in the program with values reported in Ref. 27 for those different conditions.

\subsection{Cutter Wear Rates}

To prevent thermally-accelerated wear, which is one to two orders of magnitude more rapid than ordinary abrasive wear, wearflat temperatures must be kept below $350^{\circ} \mathrm{C}$. At temperatures below this critical value, ordinary abrasive wear will occur. For this type of wear, a model developed in our earlier work [31] concludes that the volume of cutter material, $V$, worn away per unit length of hole drilled, $\ell_{h}$, is related to several design and operating parameters:

$$
\frac{d v_{w}}{d l_{h}}=\frac{2 \pi F X_{b c} C_{6} N}{R O P},
$$

where the constant $C_{6}$ is defined as the abrasive wear constant and is a function of the abrasiveness of the rock and the abrasion-resistance of the drag cutter materials. We may now define a wear ratio (WR) that describes the volumetric wear rate of each cutter relative to that of cutter 1 nearest the center of the bit. Thus

$$
W R=\frac{\left(d v_{w} / d l_{h}\right)}{\left(d v_{w} / d l_{h}\right)_{1}}=\frac{F x_{b c}}{\left(F x_{b c}\right)_{1}}
$$

This quantity provides a measure of wear uniformity among cutters that is independent of the abrasive wear constant $\mathrm{C}_{6}$.

A goal of any bit design should be to have uniform wear. If a given cutter wears significantly faster that the other cutters, it could prematurely reach the point where thermally-accelerated wear begins. Once this occurs, the cutter will rapidly attain a wearflat large enough to affect bit performance, i.e. cause greater drilling torque or bit imbalance due to larger cutter forces. Excessive forces can also cause the cutter to fracture off the bit body, which could have a catastrophic effect on bit life. More uniform wear helps ensure that no single cutter affects bit life through premature failure. If all cutters wear at the same rate, the 
utilization of expensive cutting materials is also maximized, and the efficiency of refitting bits with new cutters is improved.

The existence of a wear ratio also suggests the possibility of estimating relative cutter wear and modifying cutter geometries to determine the effects of wear on bit performance. As a bit drills a distance from point 1 to point 2, the volume of material worn from the cutter changes from $\left(\mathrm{V}_{\mathrm{w}}\right)_{1}$ to $\left(\mathrm{V}_{\mathrm{w}}\right)_{2}$, where

$$
\left(v_{w}\right)_{2}=\left(v_{w}\right)_{1}+\frac{d v_{w}}{d l_{h}} \Delta l_{h},
$$

as long as $\mathrm{dV}_{\mathrm{w}} / \mathrm{d} \ell_{\mathrm{h}}$ is constant over the drilling distance. We may then divide Eq. 35 by $^{\text {the }}$ same equation evaluated for a reference cutter $(r$ ) and define

$$
\Delta v_{w}=\left(v_{w}\right)_{2}-\left(v_{w}\right)_{1}
$$

to get

$$
\frac{\Delta v_{w}}{\left(\Delta v_{w}\right)_{r}}=\frac{d v_{w} / d l_{h}}{\left(d v_{w} / d \ell_{h}\right)_{r}}
$$

or, using Eqs. 33 and 34,

$$
\Delta v_{w}=\frac{W R}{(W R)_{r}}\left(\Delta v_{w}\right)_{r}
$$

Thus, if we specifiy the change in the wear volume for the reference cutter, $\left(\Delta \mathrm{V}_{\mathrm{w}}\right)_{r}$, the wear volumes of the other cutters can also be determined using the ${ }^{\mathrm{W}}$ computed wear ratios. If the value of the abrasive wear constant $\mathrm{C}_{6}$ is known for the rock of interest, the distance $\Delta l_{h}$ drilled in wearing away the volume $(\Delta V)$ of reference cutter material can be determined. Combining Eqs. 32 and $^{\mathrm{W}} 3 \frac{\mathrm{h}}{4}$, we obtain the result

$$
\Delta \ell_{h}=\frac{\left(\Delta v_{w}\right)_{r}{ }^{R O P}}{2 \pi\left(F x_{b c}{ }^{\prime} r C_{6} N\right.} .
$$

It is more convenient to express cutter wear in terms of dimensions that can be readily measured. It is, therefore, necessary to determine the relationships between cutter wear volume and the other wearflat dimensions. These relationships are functions of the wear mode. As discussed previously, two distinct wear modes seem to occur. Accordingly, two separate wear models are developed, one for each mode. 


\section{Hard-Rock Wear Mode}

In hard, brittle rock, the wearflat tends to develop parallel to the cutting direction. In our previous work, we showed that the length of such a wearflat may be expressed as a simple function of the wear volume [31]:

$$
L=C_{4} v_{w}^{n 4}
$$

or, alternatively, as a function of the wearflat area [27]:

$$
L=C_{5} A_{w}^{n 5}
$$

where $\mathrm{C}_{4}, \mathrm{n} 4, \mathrm{C}_{5}$, and $\mathrm{n} 5$ are functions of the cutter compact radius and backrake angle. Values of these parameters have been determined for $20^{\circ}$ backrake cutters with 0.5 -inch and 0.75 -inch diameter compacts, as listed in Table I. This analysis assumes that the wearflat is in the center of the cutting profile, i.e. $\phi_{\mathrm{w}}=\phi_{\mathrm{c}}$, but the error for cutter side wear is not large with the backrake angles generally used in PDC bit design $\left(5-20^{\circ}\right)$.

\section{TABLE I}

\begin{tabular}{|c|c|c|c|c|}
\hline $\begin{array}{l}\text { COMPACT DIAMETER } \\
\text { (in.) }\end{array}$ & $\left(i n^{1-3 n 4}\right)$ & $\mathrm{n} 4$ & $\begin{array}{c}C_{5} \\
\left.\text { in }^{1-2 n 5}\right)\end{array}$ & n5 \\
\hline 0.50 & 2.94 & 0.40 & 1.59 & 0.68 \\
\hline 0.75 & 2.72 & 0.40 & 1.38 & 0.68 \\
\hline
\end{tabular}

COEFFICIENTS IN EQUATIONS 38 AND 39

The width of the wearflat, measured at the diamond face, is

$$
w=2 \sqrt{r^{2}-(r-L \sin B)^{2}} .
$$

For the hard-rock wear mode, we may now specify a new wearflat area for the reference cutter, compute the associated new wear volume using Eqs. 38 and 39 and compare this with the old wear volume to determine $\left(\Delta V_{w}\right)$ according to Eq. $36 \mathrm{a}$. Eqs. 34 and $36 \mathrm{c}$ can then be used to compute the new wear volume, $\left(\mathrm{V}_{\mathrm{W}}\right)_{2}$, for every other cutter on the bit. From these volumes, the new wearflat length, area, and width for each cutter can calculated using Eqs. 38-40. Re-running the program with the new wear configuration 
then allows the user to assess the effects of the predicted wear pattern on bit performance.

\section{Soft-Rock Wear Mode}

In the early stages of wear in the soft-rock wear mode, the wearflat develops parallel to the cutting direction, and the wear model can therefore be assumed to be identical to that of the hard-rock wear mode. At some point, however, the length of the wearflat, L, reaches some maximum value, $\mathrm{L}$, and further wear occurs by wearing the WC-Co at an angle with respect to ${ }^{\prime}$ the cutting direction (see Figure 23). Since this angle is relatively small (only 5-10 ), the total length of the wearflat, $L_{t}$, including the portion not contacting the rock, is given approximately by the equation

$$
L_{t} \cong C_{4}\left(v_{w}\right)_{t}^{n 4}
$$

which follows from Eq. 38. Under these conditions, the total wearflat area, $\left(A_{W}\right)$, including the portion not contacting the rock, is related to $\mathrm{L}_{t}$ by the equation

$$
\mathrm{L}_{t} \cong \mathrm{C}_{5}\left(\mathrm{~A}_{\mathbf{w}}\right)_{t}^{\mathrm{n} 5}
$$

which follows from Eq. 39 .

The parameter needed for the cutting force correlation is $A_{w}$, which is the portion of the wearflat in contact with the rock. This parameter can be determined by recognizing that

$$
\left(A_{w}\right)_{t}=A_{w}+\left(A_{w}\right)_{n c}
$$

where $\left(A_{\text {f }}\right)_{n c}$ is the portion of the total wearflat that does not contact the rock. The geometry considerations discussed in Ref. 27 suggest that such a portion of the total wearflat area is simply a function of the length of that portion; i.e.,

$$
\left(\mathrm{A}_{\mathrm{w}}\right)_{\mathrm{nc}}=\left[\left(\mathrm{L}_{\mathrm{t}}-\mathrm{L}_{\max }\right) / \mathrm{C}_{5}\right]^{1 / \mathrm{n} 5} .
$$

Inserting Eqs. 42 and 44 into 43 gives the result

$$
A_{w}=\left(L_{t} / C_{5}\right)^{1 / n 5}-\left[\left(L_{t}-L_{\max }\right) / C_{5}\right]^{1 / n 5}
$$

For the purpose of developing a preliminary model for the soft-rock wear mode, it is now assumed that $\mathrm{L}_{\mathrm{m}}=0.09$ inch, which is the maximum length of the portion of the wearflat in contact with the rock that was measured in the experimental cutter test program with field-worn cutters. 
The wear procedure for the soft-rock wear mode then is to specify a new wearflat area, $A_{W}$, for the reference cutter and calculate $L_{t}$ with Eq. 45, using an iterative procedure. If $\mathrm{L} \leq \mathrm{L}_{\mathrm{max}}$, then $\mathrm{V}_{\mathrm{W}}$ is calculated using Eq. 41, and the new wear volume is compared with the old wear volume to determine $\left(\Delta \mathrm{V}_{\mathrm{w}}\right)_{\mathrm{r}}$ according to Eq. $36 \mathrm{a}$.

Eqs. 34 and $36 \mathrm{c}$ are next used to determine the new wear volume for each cutter, and Eq. 41 is used to determine the new total wearflat length, $\mathrm{L}_{t}$. For those cutters with $\mathrm{L}_{\mathrm{L}} \leq \mathrm{L}_{\mathrm{m}}$, the new length of the wearflat portion in contact with the rock, $E$, is ${ }^{-}$set to $\mathrm{L}_{t}$, and the procedure described for the hard-rock wear mode is used to determine the new wearflat area and width in the advanced wear state. For cutters with $\mathrm{L}_{f}>\mathrm{L}_{\text {max }}$ Eq. 45 is used to determine the new wearflat area $A$. The length of the maxearflat portion in contact with the rock for such cutters is

$$
\mathrm{L}=\mathrm{L}_{\max },
$$

and the width of the wearflat at the diamond face is

$$
w \approx 2 \sqrt{r^{2}-\left(r-L_{t} \sin B\right)^{2}}
$$

A loop is written in the program to allow the user to repeat the wear process described above for either wear mode as many times as desired. The user can run the program for multiple bit wear states ranging from sharp to severely worn. This provides a means for obtaining predicted results necessary for assessing drilling performance over the life of the bit.

More familiarity with the computer code can be attained by studying the comment statements contained in the code listing in Appendix $B$. The remainder of the text of this report concentrates on demonstrating the use of the code and drawing some general conclusions based on the predictions of the code. 


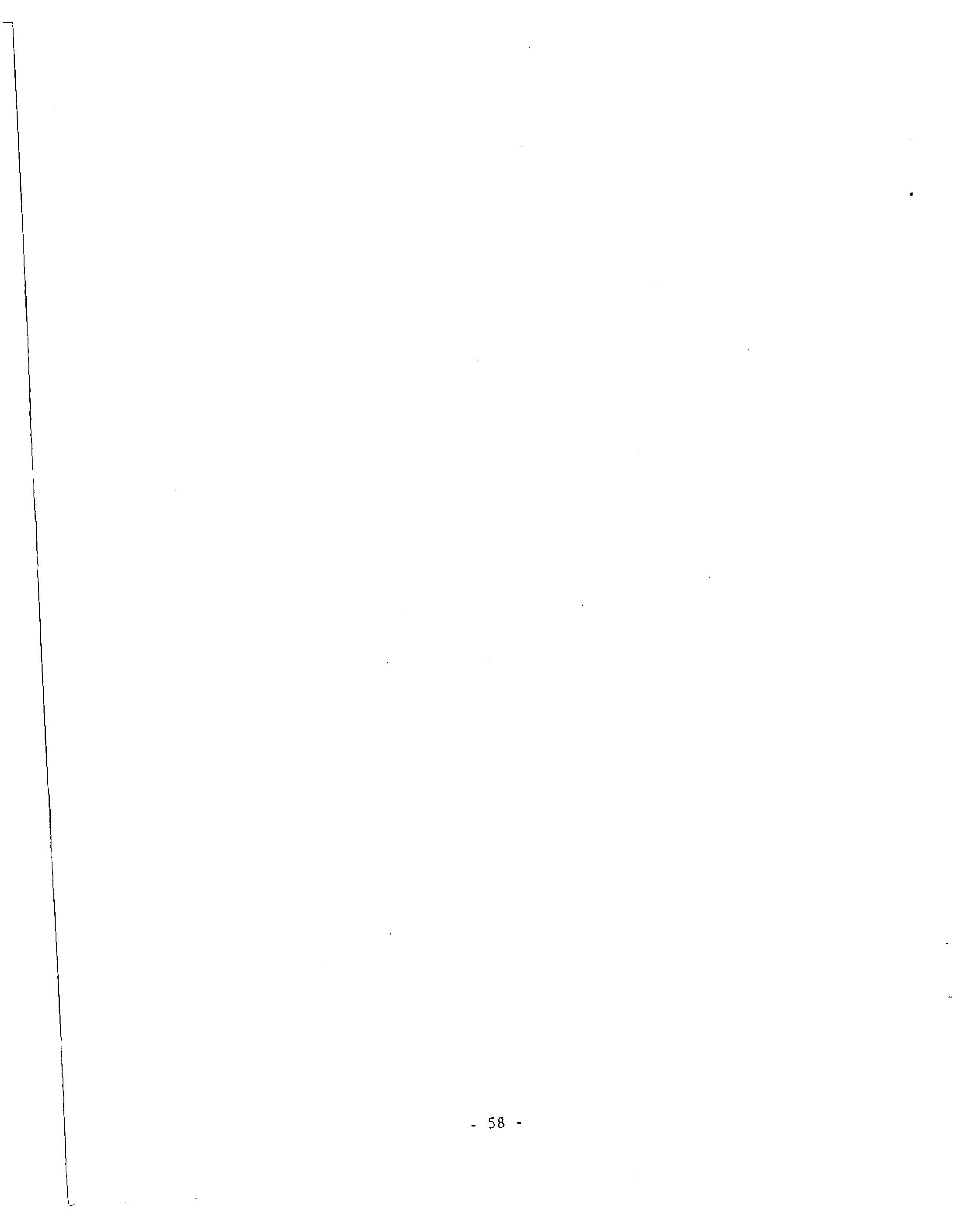




\subsection{DEMONSTRATION OF THE USE OF PDCWEAR}

In this section, the use of PDCWEAR is demonstrated by analyzing the bit design shown in Figure 30. This design is for an 8.5 inch-diameter PDC bit with 21 cutters having 0.75 -inch diameter compacts. The design is intended for hard-rock drilling, so the bit consequently has a relatively flat profile. The positions of the cutters assumed for this analysis are listed in Table II. Assumed operating conditions for the demonstration analysis are listed in Table III.

The following subsections describe the input parameters required by the code, steps required to run the program, program output, and the results obtained with the demonstration analysis.
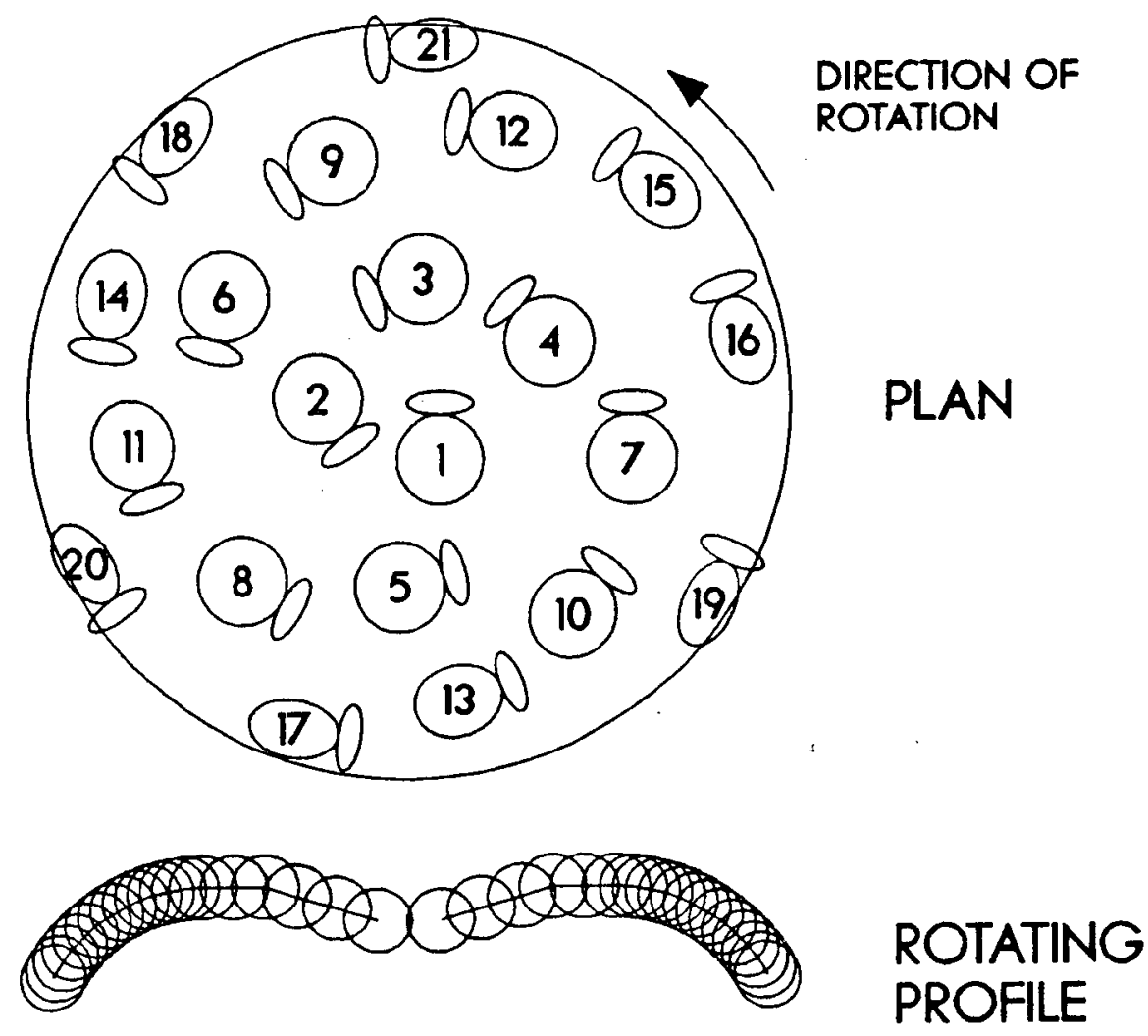

Figure 30 - Schematic of 8-1/2 inch bit design used in the demonstration analysis. 
TABLE II

CUTTER POSITIONS IN DEMONSTRATION ANALYSIS *

\begin{tabular}{rrrrr} 
CUTTER & \multicolumn{1}{c}{$\mathrm{R}$} & $\boldsymbol{\theta}$ & $\mathrm{H}^{\prime}$ & $\begin{array}{c}\phi_{\mathrm{C}} \\
\end{array}$ \\
& (in) & (deg) & (in) & (deg) \\
\hline 1 & 0.350 & 0.0 & 0.625 & -15.0 \\
2 & 0.800 & 215.0 & 0.760 & -10.0 \\
3 & 1.250 & 110.0 & 0.895 & -5.0 \\
4 & 1.600 & 45.0 & 1.000 & -2.0 \\
5 & 1.950 & 285.0 & 1.000 & 0.0 \\
6 & 2.300 & 165.0 & 1.000 & 2.0 \\
7 & 2.495 & 0.0 & 0.990 & 5.0 \\
8 & 2.635 & 240.0 & 0.970 & 10.0 \\
9 & 2.775 & 120.0 & 0.940 & 15.0 \\
10 & 2.915 & 320.0 & 0.898 & 20.0 \\
11 & 3.055 & 200.0 & 0.844 & 25.0 \\
12 & 3.195 & 80.0 & 0.776 & 30.0 \\
13 & 3.335 & 290.0 & 0.694 & 35.0 \\
14 & 3.475 & 170.0 & 0.594 & 40.0 \\
15 & 3.615 & 50.0 & 0.473 & 45.0 \\
16 & 3.735 & 20.0 & 0.347 & 47.5 \\
17 & 3.835 & 260.0 & 0.222 & 50.0 \\
18 & 3.915 & 140.0 & 0.104 & 52.5 \\
19 & 3.975 & 335.0 & 0.000 & 55.0 \\
20 & 3.975 & 215.0 & 0.000 & 55.0 \\
21 & 3.975 & 95.0 & 0.000 & 55.0
\end{tabular}

* See Figure 31 for definition of cutter position parameters. 
TABLE III

BIT OPERATING CONDITIONS IN DEMONSTRATION ANALYSIS

PARAMETER

Rock thermal conductivity, $\mathrm{k}_{2}$

Rock thermal diffusivity, $\chi_{2}$

Rock/cutter friction coefficient,

Worn cutter drag coefficient, Sharp cutter drag coeffcient,

Worn Type A cutter (no jets) correlation constant, $C_{1}$

Worn Type A cutter (no jets) correlation exponent, nf

Worn Type B cutter (w/jets) correlation constant, $C_{1}$

Worn Type B cutter (w/jets) correlation exponents, $\frac{1}{n} 1$

Sharp Type A cutter (no jets) correlation constant, $C_{2}$

Sharp Type A cutter (no jets) correlation exponent, n2

Sharp Type B cutter (w/jets) correlation constant, $C_{3}$

Sharp Type B cutter (w/jets) correlation exponent, $n 2$

Abrasive Wear constant, $\mathrm{C}_{6}$

Bit rotary speed, $\mathrm{N}$

Downhole cooling fluid temperature, $T_{f}$

Specified bit penetration rates, ROP $f$
VALUE

$1.300 \mathrm{Bty} / \mathrm{hr} \mathrm{ft}^{\mathrm{o}_{\mathrm{F}}}$

$0.033 \mathrm{ft}^{2} / \mathrm{hr}$

0.070

0.550

0.750

$1.34 \times 10^{5} \mathrm{psi}$ in $^{0.42}$

0.42

$3.26 \times 10^{5} \mathrm{psi} / \mathrm{in}^{0.93}$

$2.55 \times 10^{4} 1 \mathrm{bf} / \mathrm{in}^{1.64}$

1.64
$2.55 \times 10^{4} 1 \mathrm{bf} / \mathrm{in}^{1.64}$

1.64

$6.89 \times 10^{-13}$ in $^{2} / 1 \mathrm{bf}$

100 RPM

$80^{\circ} \mathrm{F}$

$10,20,30,40,50 \mathrm{ft} / \mathrm{hr}$ 


\subsection{Input Parameters}

Input parameters are contained in three data files: bit design data, initial cutter wear configurations, and operating conditions. This segregation allows flexibility in running the code. For example, it is possible to run different bit designs for different sets of wear and operating conditions by simply specifying different combinations of input files.

The input guide presented in Appendix D details the input parameters and their required formats, and it also provides guidance for determining appropriate input values. To aid in properly formatting the data, a utility program, FORMAT, has been written and is listed in Appendix E. When this interactive program is run, the user is requested to enter values for the various cutter parameters, and the data are written to files in the proper format. For the demonstration analysis, the input files are named BITDES.DAT, WEARCF.DAT, and OPCOND.DAT .

The bit design data file, BITDES.DAT, requires the following information for each cutter: cutter compact radius, radial position, angular position, longitudinal position, inclination angle, backrake angle, convective cooling coefficient, and type of cutter. For the demonstration bit design, these parameters are listed in Appendix F, Table F-1, in the proper format. Some of these parameters are defined in Figure 31.

The cutter positions for the demonstration bit design are chosen such that the cutters lie in three spiral rows on the bit face, as shown in Figure 30 . The specified inclination angles allow the cutters to be mounted in holes drilled roughly perpendicular to the bit body at each cutter location. Cutter radii, backrake angles, and convective cooling coefficients are entered for each cutter as values relative to nominal values, which are also specified by the user. For the demonstration bit design, we specify 0.75 -inch diameter (0.375-inch radius) cutters, all employing a 20 backrake angle and having convective cooling coefficients of $1700 \mathrm{Btu} / \mathrm{hr} \mathrm{ft}^{20} \mathrm{~F}$, a value typical for water-based mud drilling.

The sign of the relative convective cooling coefficient is used to identify the type of each cutter. A positive value of the relative convective cooling coefficient indicates that the cutter is of type $A$ and is governed by the penetrating stress and force correlations specified for that type cutter. A negative value of the relative cooling coefficient indicates that the cutter is of type $B$ and is governed by a different set of input correlation constants. For the demonstration bit, we initially set all cutters to type $A$ and assume that type A cutters are cutters without waterjet assistance. We later examine the effects of waterjet assistance for selected cutters, defining the type B correlation constants as those measured in the laboratory with waterjet assistance.

The second input data file for this analysis is WEARCF.DAT. This file contains information describing the initial state of wear assumed for each cutter. The area, width, and length of each cutter wearflat must be specified. The user may specify new cutters, where all three parameters for each cutter are zero; or finite values of the wear dimensions may be entered 

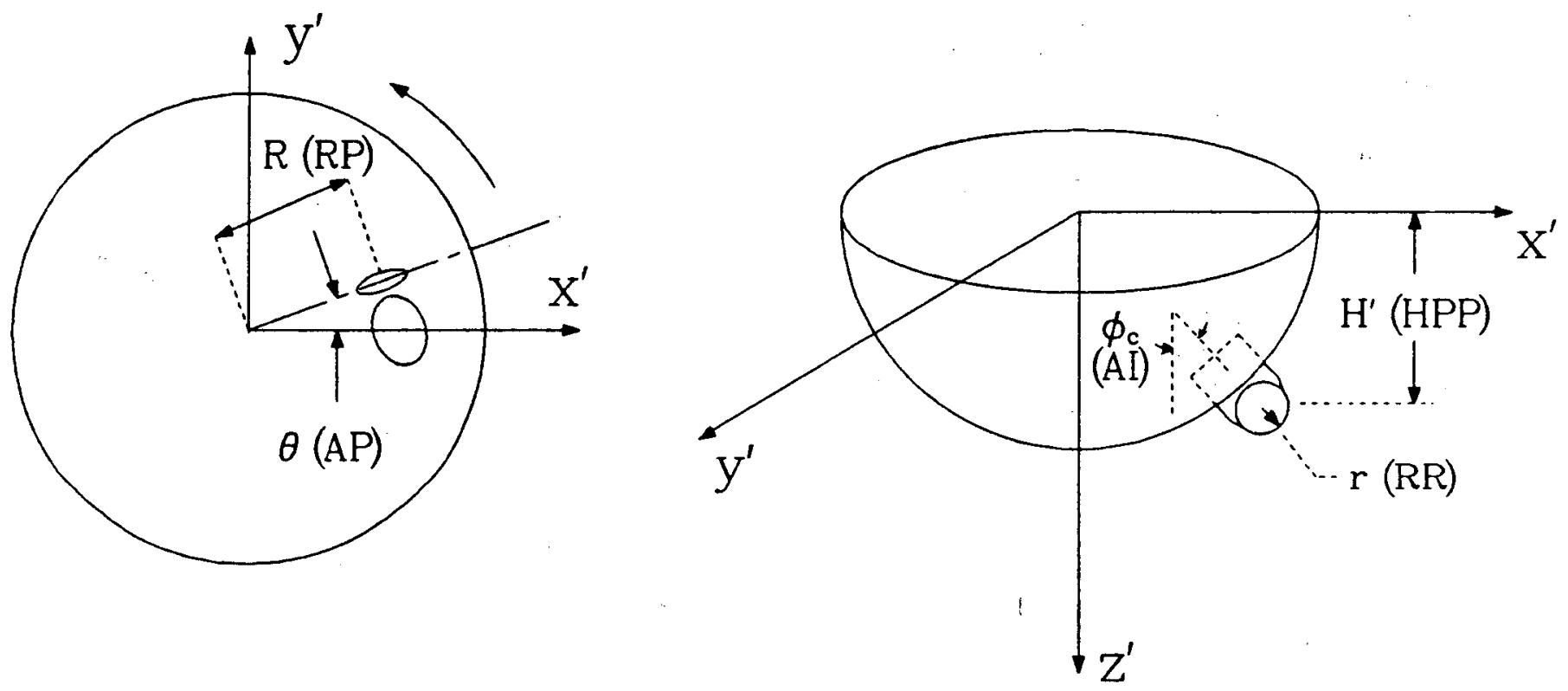

Figure 31 - Definition of several bit design parameters required as input for PDCWEAR. Each analysis variable name is followed by the computer code variable name in parentheses. 
to simulate drilling with a worn bit. These wearflat dimensions may result from specific wear models, or the user may wish to use measurements of wearflats on an actual bit. In any case, the three dimensions refer to the portion of the wearflat in direct contact with the rock surface being cut.

For the demonstration bit, we specify initially sharp cutters, as shown in the proper format in Table F-2 of Appendix F. The use of worn cutters is demonstrated by allowing the cutters to wear according to the wear model and algorithm incorporated into the computer program.

The final input data file, OPCOND.DAT, contains data describing the intrinsic drillability of the rock and the assumed operating conditions. The values chosen for the demonstration bit analysis are shown in the proper format in Table F-3.

The rock being drilled is Sierra White granite. In a water-based fluid environment, the friction coefficient between the cutter and this type of rock has been measured as 0.07 , compared with a value measured under dry conditions of 0.16 [19]. We wish to simulate a water-drilling environment, so we choose 0.07 for the demonstration analysis.

The data of Figures 13 and 15 suggest an average drag coefficient of about 0.64 for worn cutters and 0.84 for sharp cutters when cutting dry Sierra White granite. We may estimate the drag coefficients for both cutter types when cutting Sierra White granite under wet conditions by reducing the dry values by the same amount as the friction coefficient is reduced by the presence of water (Eq. 7). We thus arrive at the drag coefficients of 0.55 and 0.75 used in the demonstration analysis.

The cutter penetrating stress and force correlation constants and exponents 1 isted in Tables III and F-3 are taken from Figures 10, 12, and 16 for worn type $A$, sharp type $A$, and worn type $B$ cutters, respectively. No data have been obtained in this study with sharp cutters and waterjet assistance since cutter forces are already very small with sharp cutters. Accordingly, the constant and exponent for sharp type B cutters are assumed to be the same as those for sharp type A cutters.

Data with which to determine the abrasive wear constant, $C_{6}$, for Sierra White granite were not available. As a result, the abrasive wear constant used in the demonstration analysis was derived from data for Jack Fork and Nugget sandstones [19,33], using Eq. 33. Although both of these sandstones are relatively hard and abrasive, it is unlikely that they are as abrasive as Sierra White granite. As a result, the predicted length of hole drilled in reaching a given wear configuration in the demonstration analysis is probably greater than that which would actually be achieved with Sierra White granite.

We choose for the demonstration bit analysis a rotary speed of 100 RPM. We wish to simulate drilling under atmospheric laboratory conditions, so we assume a cooling fluid temperature of only $80^{\circ} \mathrm{F}$. Five penetration rates are specified for the demonstration analysis: $5,10,20,40$, and $50 \mathrm{ft} / \mathrm{hr}$. These rates cover the practical range of interest for this type of rock. 


\subsection{Running the Program}

PDCWEAR is run interactively, with the user specifying the files to be read, files to be written, and length of borehole drilled during the analytical simulation. A general description of the session for the demonstration analysis is provided below.

The first request from PDCWEAR is the name of the input file containing the bit design data. File names up to 11 characters in length, including the file extension, can be used. When an acceptable name has been entered, the program reads the file and stores the bit design data. It then gives the user the opportunity to display the input data at the user's terminal.

A similar pattern is repeated for the cutter wear configuration data file and the operating parameter data file. As the data are read from the specified files, the program checks the data to determine whether they are within expected ranges for each variable. If any data are out of range, the program notifies the user, requests that corrections be made, and terminates the session. Once all input data have been accepted by the program, it requests the name of the output file for storing results.

The program then requests the user to specify the value of the parameter NRAY. This parameter determines the precision to which the program calculates the results. NRAY determines the number of elemental lengths $\Delta \mathrm{x}$ into which the $\mathrm{x}$-axis is divided by the numerical integration routine that computes cutter interaction. To ensure an adequately fine division of the $x$-axis for even the smallest cutter used in any given bit design, NRAY is defined as the number of elemental lengths used over the width of the smallest cutter. Based on the specified value of this parameter and the diameter of the smallest cutter, the program calculates a value of $\Delta x$ and uses this value at all $x$ across the face of the bit. A value of 100 is a reasonable value for NRAY. Lower values may be used to speed up calculations at the expense of precision. Values of NRAY larger than 100 may be used for final analysis of a bit design in order to confirm that greater precision would not change the results. For the demonstration analysis, a value of 100 was initially used for NRAY. The program was then re-run using a value of 500 , but the difference in results was insignificant.

After specification of NRAY by the user, the program begins the calculations. For the initial wear configuration specified in WEARCF.DAT and the first penetration rate specified in OPCOND.DAT, the program iterates on the radial wear angle for each cutter until convergence. If convergence is not attained within 30 iterations, the program displays the latest two iteration values for each cutter wear angle and gives the user the opportunity to proceed with the latest values or to terminate the program. Generally a failure to converge indicates that the user has not used a sufficiently large value of NRAY. In most cases, however, continuing with the non-converged wear angles will not cause significant inaccuracies in the algorithms used to compute total bit performance parameters.

Following the radial wear angle determinations, the program computes

the rock removal volume, cross-sectional area of cut, effective depth of cut, penetrating force, drag force, wearflat temperature, and wear ratio for 
each cutter. These detailed data are written to the specified output file. In addition, the following data are computed and written to both the output file and the user's terminal: weight-on-bit, drilling torque, bit side forces, bit bending moments, maximum wearflat temperature, and maximum wear ratio across the bit.

Before repeating the calculations for the next specified penetration rate, the program allows the user to open several files into which data are written in a format suitable for plotting. Cutter plot files are formatted as shown below:

Bit Design Data File Name

Wear Configuration Data File Name

Operating Conditions Data File Name

ROP

$1, Q(1)$

$2, Q(2)$

$3, Q(3)$

(1)

$\mathrm{n}_{\mathrm{c}}, \mathrm{Q}\left(\mathrm{n}_{\mathrm{c}}\right)$

where $Q(j)$ represents the computed quantity associated with the $j$ th cutter. The following cutter plot files may be saved on disk:

(1) FVSCN.DAT contains the penetrating force for each cutter for the current ROP (i.e., $Q(1)=F$ for cutter 1 , etc.) ;

(2) DEFVSCN.DAT contains the effective depth of cut for each cutter;

(3) TWVSCN.DAT contains mean wearflat temperatures for each cutter;

(4) WRVSCN.DAT contains the wear ratio for each cutter;

(5) VRVSCN.DAT contains the rock cutting volume for each cutter; and

(6) ARVSCN.DAT contains the cross-sectional area of cut for each cutter.

After data have been written to the cutter plot files, the program repeats the calculations for the next specified penetration rate. Data are again written to the cutter plot files, and the process is repeated for the next specified penetration rate. (The program is written such that once a cutter plot file is opened, the appropriate data are automatically written to the opened plot file for each subsequent penetration rate.)

After results have been computed for all specified penetration rates, the program writes summary data to the output file for the current wear configuration and displays the same summary data at the user's terminal. It then gives the user the opportunity to write this data to bit plot files. The format of these files is: 
Bit Design Data File Name

Wear Configuration Data File Name

Operating Conditions Data File Name

$\mathrm{ROP}(1), \mathrm{Q}(1)$

$\operatorname{ROP}(2), Q(2)$

$\mathrm{ROP}(3), \mathrm{Q}(3)$

$\operatorname{ROP}(4), Q(4)$

$\operatorname{ROP}(5), Q(5)$

where $Q(i)$ represents the computed quantity associated with the $i$ th penetration rate, $\operatorname{ROP}(i)$. The following bit plot files may be saved on disk:

(1) WOBVROP.DAT contains the weight on bit data computed at each penetration rate (i.e., $Q(1)=$ WOB computed at first specified penetration rate, etc.);

(2) TRQVROP.DAT contains the drilling torque at each penetration rate;

(3) SFRVROP.DAT contains the resultant bit side forces at each penetration rate;

(4) MXVROP.DAT contains the bending moments about the $\mathrm{x}^{\prime}$-axis at each penetration rate; and

(5) MYVROP.DAT contains the bending moments about the $\mathrm{y}^{\prime}$-axis at each penetration rate.

After the bit performance data are written to the selected files, analysis for the intial wear configuration is complete. The program is then ready to consider new wear configurations for the cutters. The program stores the wear ratios computed at the third penetration rate of the five rates specified and uses these values in computing relative cutter wear. This implies that at least three penetration rates must be specified when wear calculations are to be made. For the demonstration analysis, the wear ratios computed at $30 \mathrm{ft} / \mathrm{hr}$ were used by the program in computing cutter wear.

In order to calculate new wear configurations for all cutters, the program requires the user to select the soft-rock or hard-rock wear mode and to guide the wear process by specifying a new wearflat area for one of the cutters. For the demonstration analysis, the hard-rock wear mode was selected.

The program then requests the user to specify a new, larger wearflat area for the cutter with the largest wearflat at that point in the analysis. In the case of initially sharp cutters, where all wearflat areas are zero, the program uses the highest-number cutter as the reference cutter (cutter 21 in the demonstration analysis). After the user specifies a new wearflat area for the reference cutter, the program computes the length of hole that the bit must drill in wearing the reference cutter from its current wear configuration to the new specified configuration. (This hole length is based on the third penetration rate specified in the operating conditions input file.) The program then displays the change in hole length and gives the user a chance to change the new wear configuration for the reference cutter. As a result, the user can monitor the cumulative hole length in order to obtain predictions after any given drilling distance. Once the user is satisfied with the new reference cutter wear configuration, the 
program computes new wear configurations for all other cutters, based on the wear ratios. It then requests the name of the file where the new wearflat dimensions are to be stored. This file can be used for later reference or for re-starting the program as the initial wear configuration file. If the user does not wish to proceed with the new configuration, the program allows the user to terminate the session.

If the user chooses to continue the session, the program repeats the bit performance calculations for the new wear configuration at all specified penetration rates. This process is repeated as many times as the user specifies, or until the cutter wearflat dimensions grow to the point that the equations developed in section 3.0 no longer describe the wearflat geometry. The program monitors the wear and will not allow the user to specify a wearflat area that is not valid. For consistent results, the same wear mode should be selected for all wear increments in a given analysis.

Since the wear ratio is a function of the wear configuration of each cutter, small increments in wearflat area growth are recommended. In the early stages of bit wear, increments of 0.005 in $^{2}$ or smaller are recommended. At later stages of wear, increments of 0.010 in can be used, but the resulting change in drilled borehole length and maximum cutter wear ratios should be monitored to ensure that large changes in these parameters do not occur in any single increment of wear. Larger wear increments than those recommended could lead to significant inaccuracies in the numerical integration routine used for simulating cutter wear, particularly during the early stages of cutter wear. By interactively specifying new wearflat areas, the user can monitor the bit wear, bit performance, and cumulative borehole length. The session can be terminated when the user determines that the bit is worn out, based on either cutter wearflat areas, cutter temperatures, or excess WOB or drilling torque.

For the demonstration analysis, the following wearflat areas were specified for the reference cutter at successive points over the life of the bit: $0.002,0.010,0.015,0.020,0.025,0.030,0.035,0.040,0.050,0.060$, $0.070,0.080,0.090,0.100,0.110,0.120,0.130,0.140$, and 0.150 in $^{2}$. The analysis was re-run by specifying increments of 0.001 in from 0.0 to 0.040 in , and the bit performance predictions were within 28 of those for the larger specified increments in wearflat area for the reference cutter.

\subsection{Tabulated Program Output}

Part of the output file for the demonstration bit analysis is listed in Appendix G. The program first prints the date and time of the computer run and then the name and contents of the files containing the bit design parameters, wear configurations, and operating conditions.

The computed results are listed next. For each penetration rate requested, the program lists three pages of results. The first of these contains the geometry-dependent results: the cutting order, the cutting height, the location of the center of each cutter wearflat, the cutter wear angle, volume of cut, cross-sectional area of cut, and effective depth of cut for each cutter. 
The second page of computed results lists the rock-dependent results: penetrating force, drag force, vertical and radial components of the penetrating force, wearflat temperatures, and wear ratio for each cutter. In cases where a wearflat temperature exceeds the critical level of $350^{\circ} \mathrm{C}$, the wear ratio for that cutter is flagged by setting it to a negative value. The program also warns the user at the terminal that one of the cutters experiences thermally-accelerated wear. Wear calculation may continue under such conditions, but the results may not be accurate since a much larger abrasive wear constant is operative under such conditions than under normal wear conditions.

The third page of computed results for each penetration rate contains integrated forces and moments for the bit: WOB, drilling torque, side force in the $x^{\prime}$ and $y^{\prime}$ directions, resultant side force, and bending moments about the $\mathrm{x}^{\prime}$ and $\mathrm{y}^{\prime}$ axes.

The results for the demonstration analysis are discussed in the next subsection.

\subsection{Discussion of Baseline Analysis Results}

The results shown in the output file in Appendix $G$ are summarized in Figures 32-40. These figures were produced by saving appropriate plot files while running PDCWEAR. The data were then edited and formatted for an available graphics program. Similar editing can be done for any graphics program available to the user; or the user could modify the appropriate statements in SUBROUTINE DATPRI of PDCWEAR so that plot data could be output in a format to better suit any available graphics program.

With the baseline bit design used in the demonstration analysis, cutter 15 wore at a higher rate than any other cutter. The growth of this cutter's wearflat area is plotted in Figure 32 as a function of the computed footage drilled by the bit. We designate three points along this curve as reference wear conditions for the bit: "sharp" when all cutter wearflat areas are zero; "worn" when the reference cutter wearflat area is $0.040_{2}$ in ; and "worn out" when the reference cutter wearflat area is 0.100 in $^{2}$. The results indicate that the bit will drill $460 \mathrm{ft}$ before reaching the designated "worn" stage, and it will drill $975 \mathrm{ft}$ before it is worn out.

The distribution of cutter wear for the baseline analysis in each of the designated stages of bit wear is shown in Figure 33. Note that with this design, cutters near but not on gage tend to experience the greatest wear (cutters 13-16). The gage cutters (19-21) wear slightly less than those on the leading face of the bit (cutters 4-6).

Baseline cutter and bit performance parameters in each of the bit wear stages are plotted in Figures 34-40. In Figure 34, the predicted penetrating forces are shown. When the bit is sharp, the penetrating forces tend to decrease from the center of the bit out toward gage. This is caused 


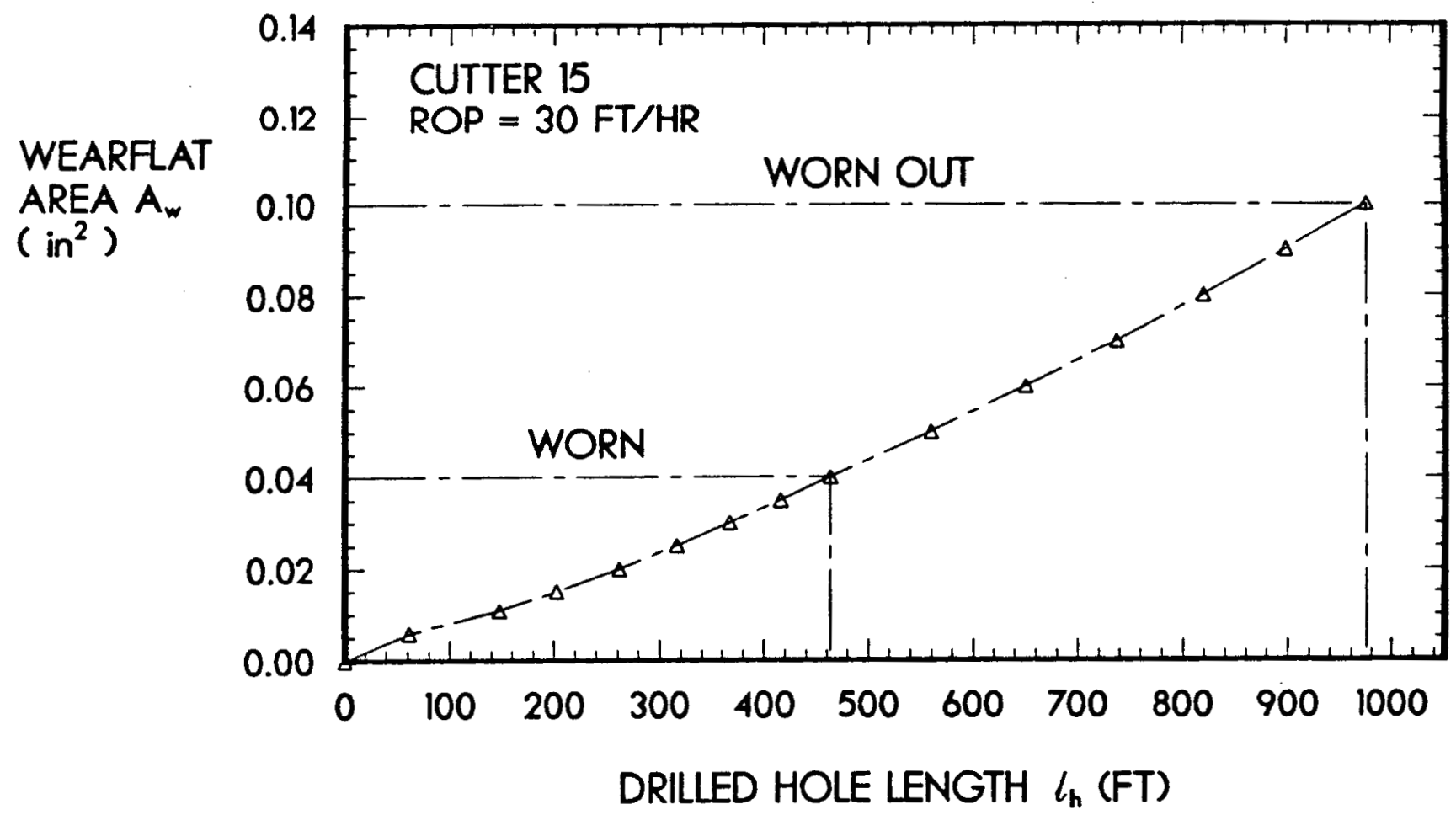

Figure 32 - Predicted wearflat growth of cutter with highest wear rate in the demonstration analysis. 


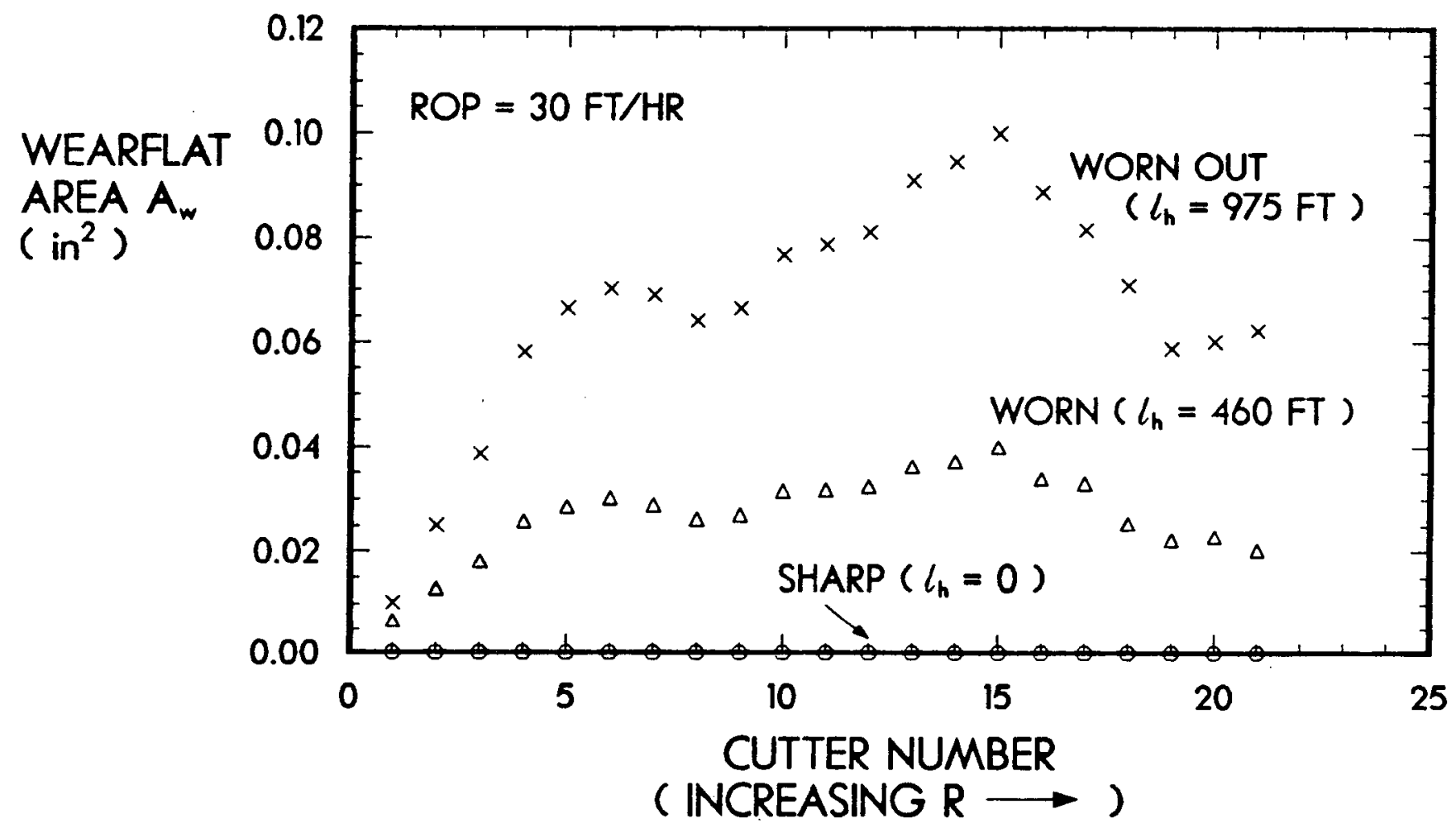

Figure 33 - Predicted wear distribution across bit in the demonstration analysis at designated stages of bit wear. 


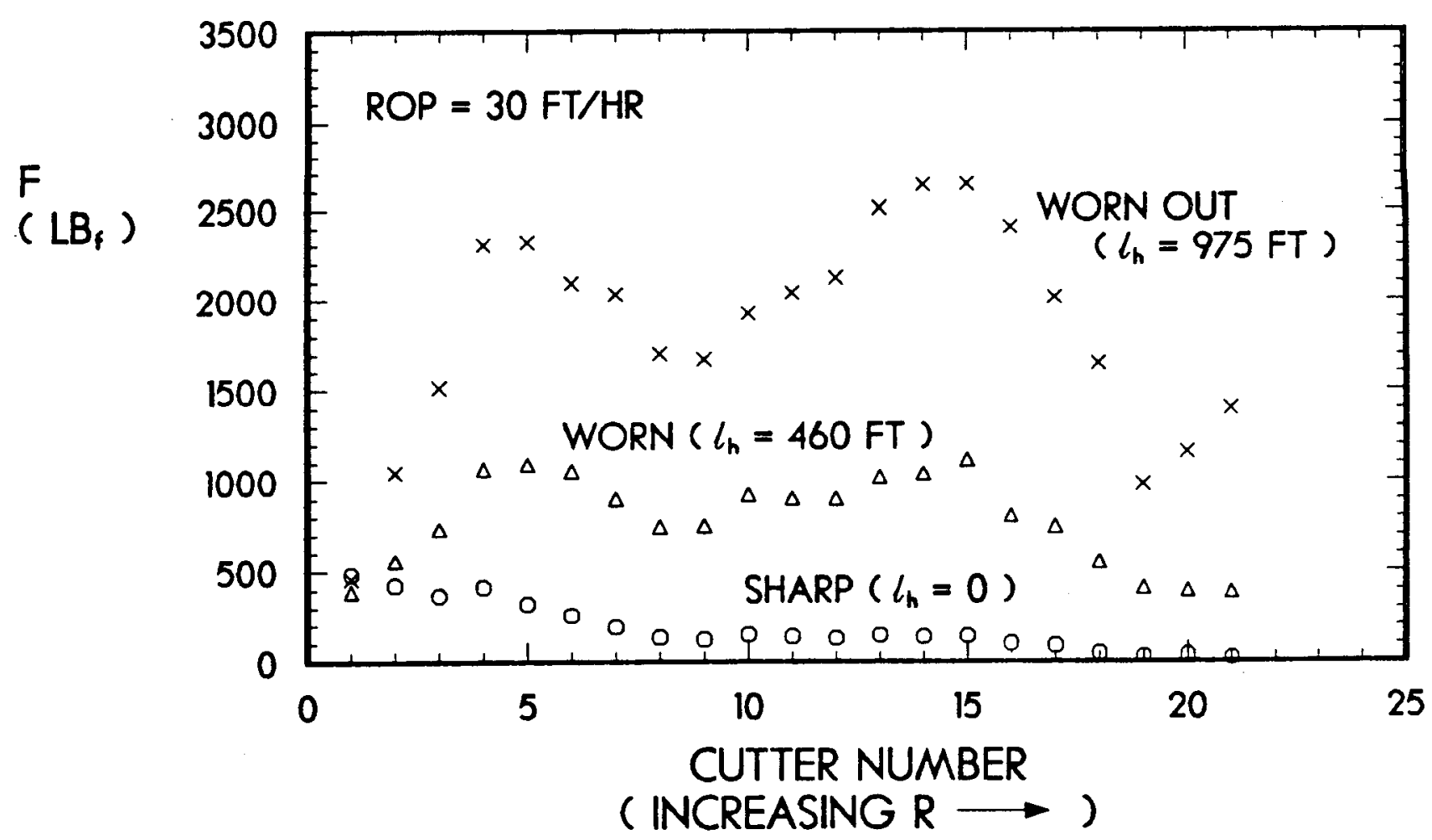

Figure 34 - Predicted cutter penetrating forces in the demonstration analysis. 


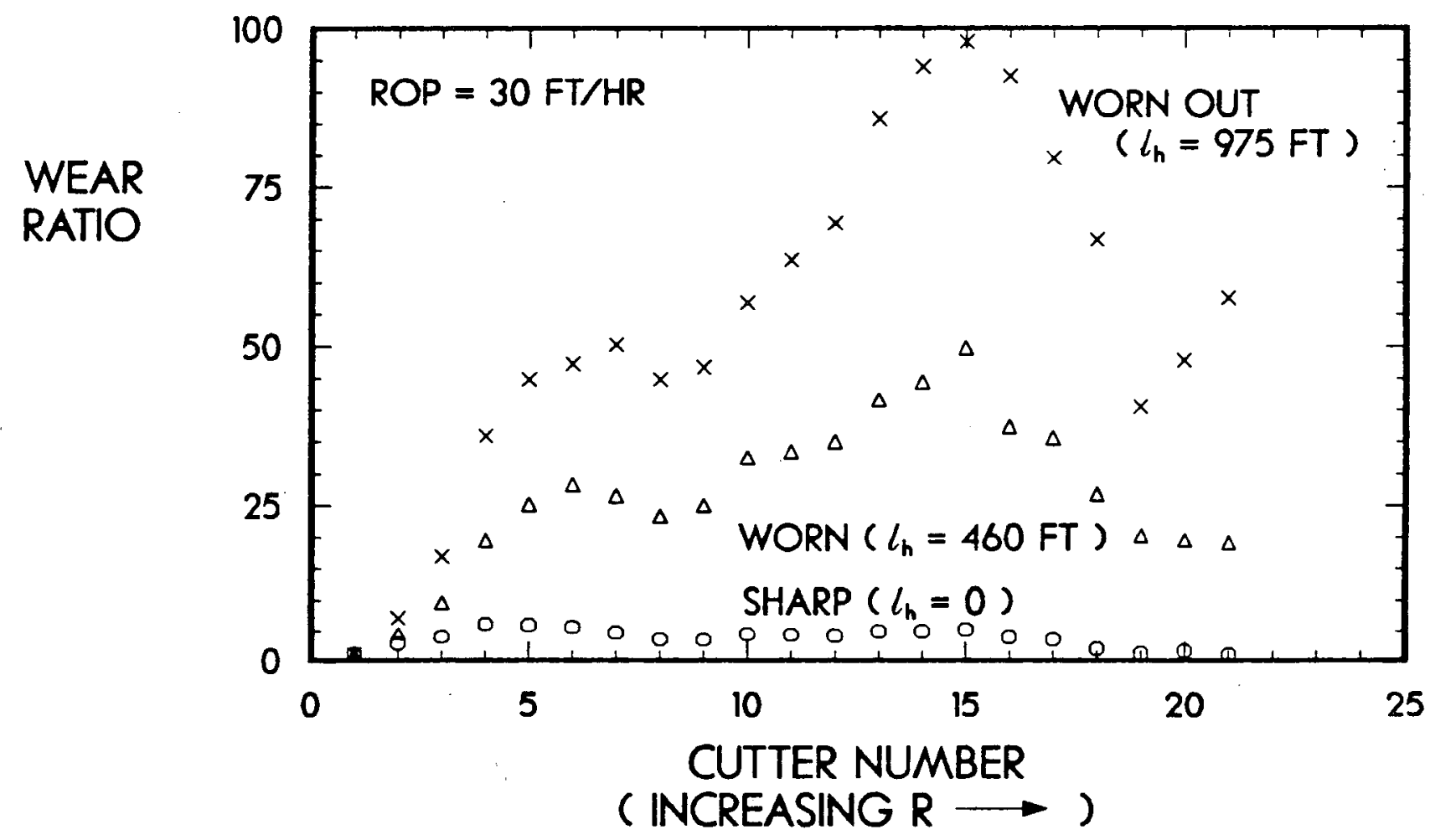

Figure 35 - Predicted cutter wear ratios in the demonstration analysis (wear ratio = 1 for cutter 1 by definition). 


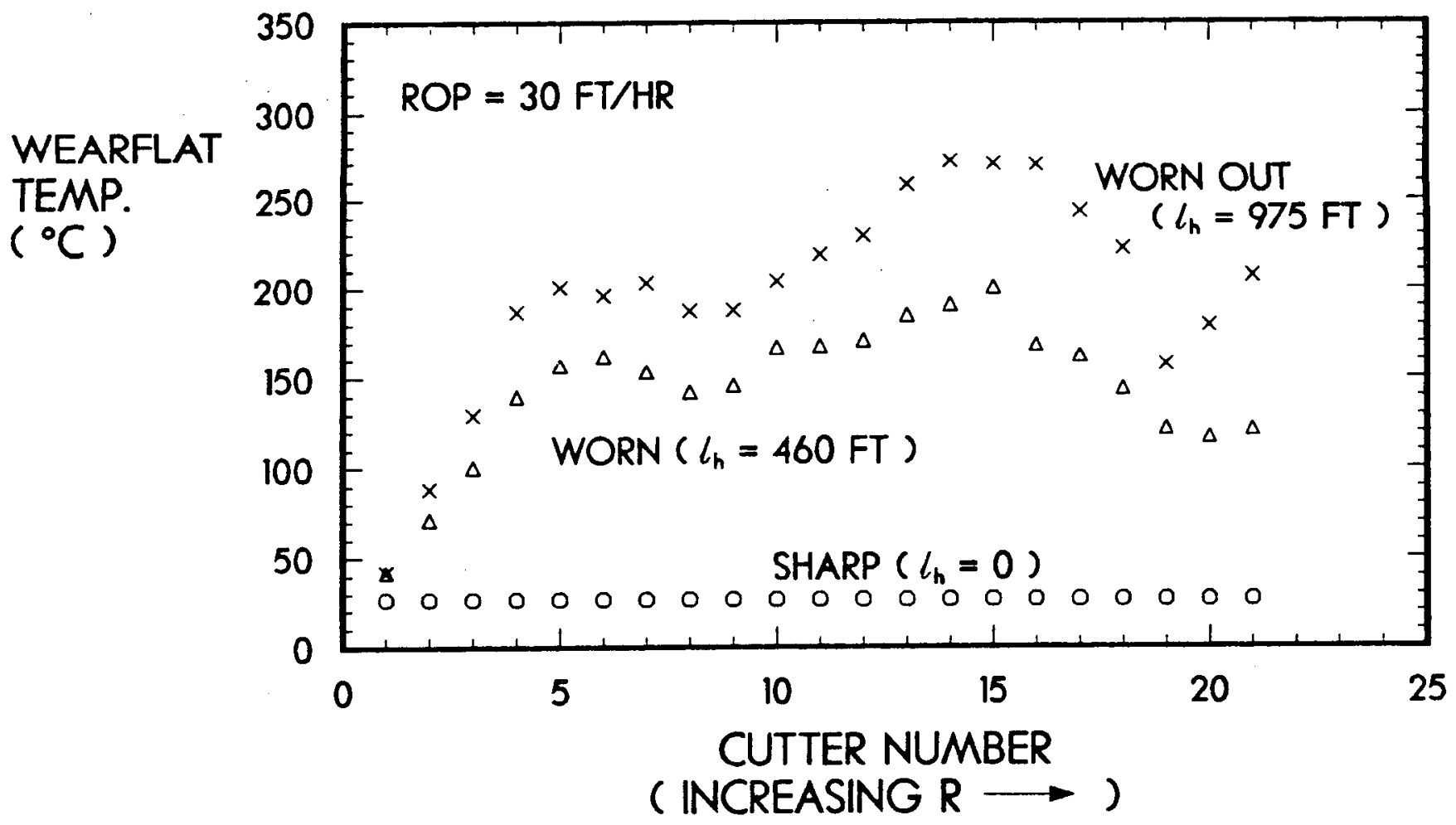

Figure 36 - Predicted cutter wearflat temperatures in the demonstration analysis. 


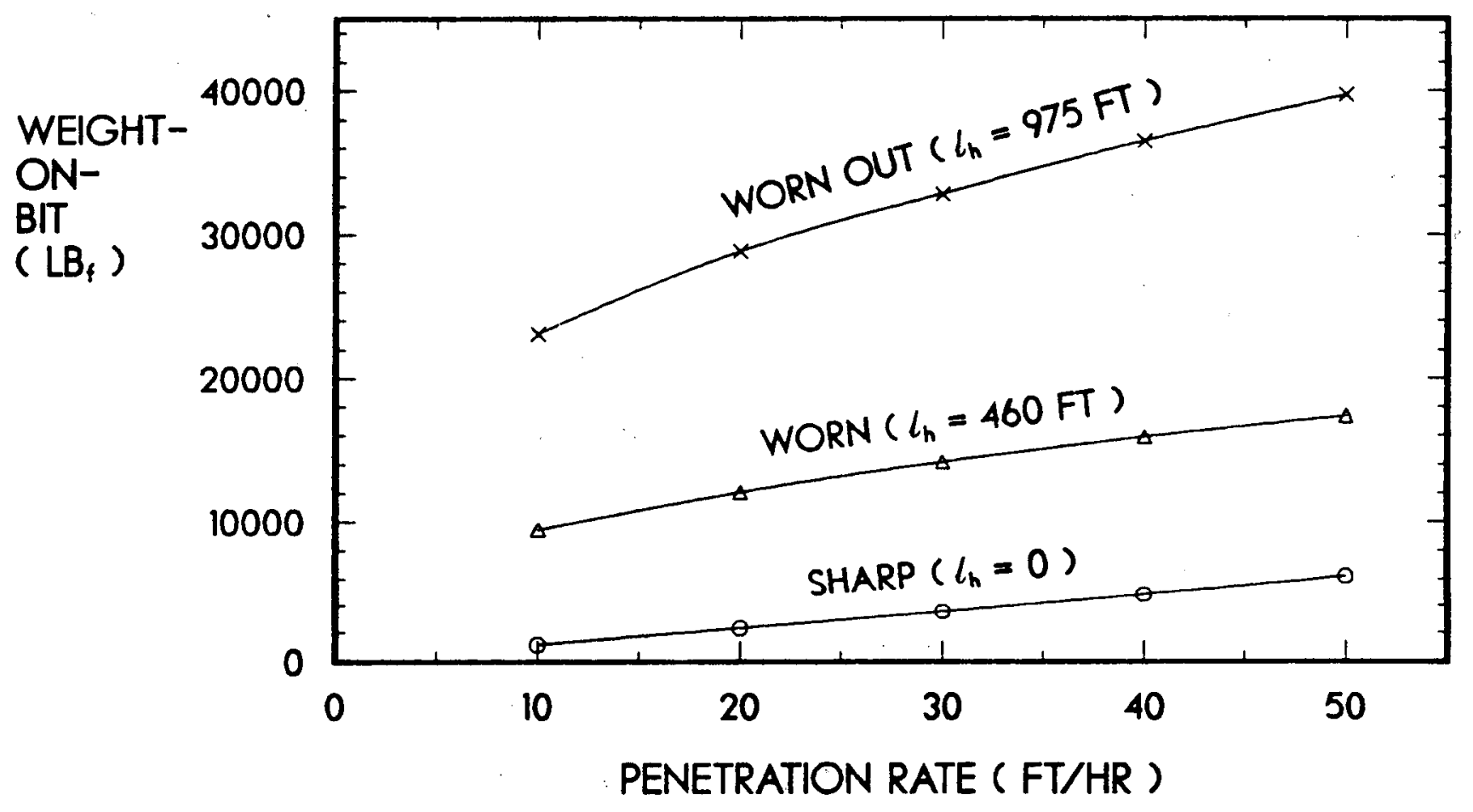
Figure 37 - Predicted WOB at specified penetration rates in the
demonstration analysis. 


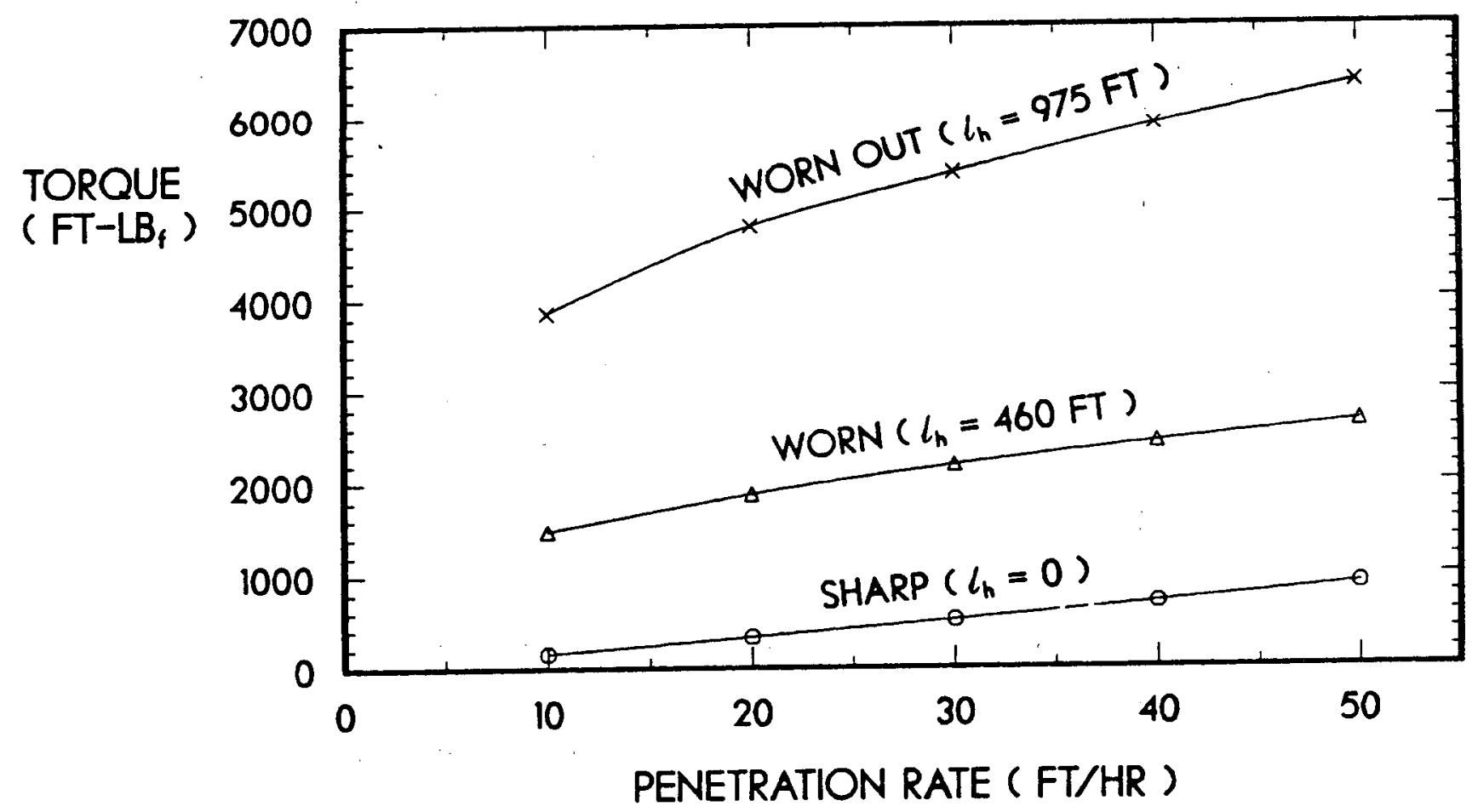

Figure 38 - Predicted drilling torque at specified penetration rates in the demonstration analysis. 


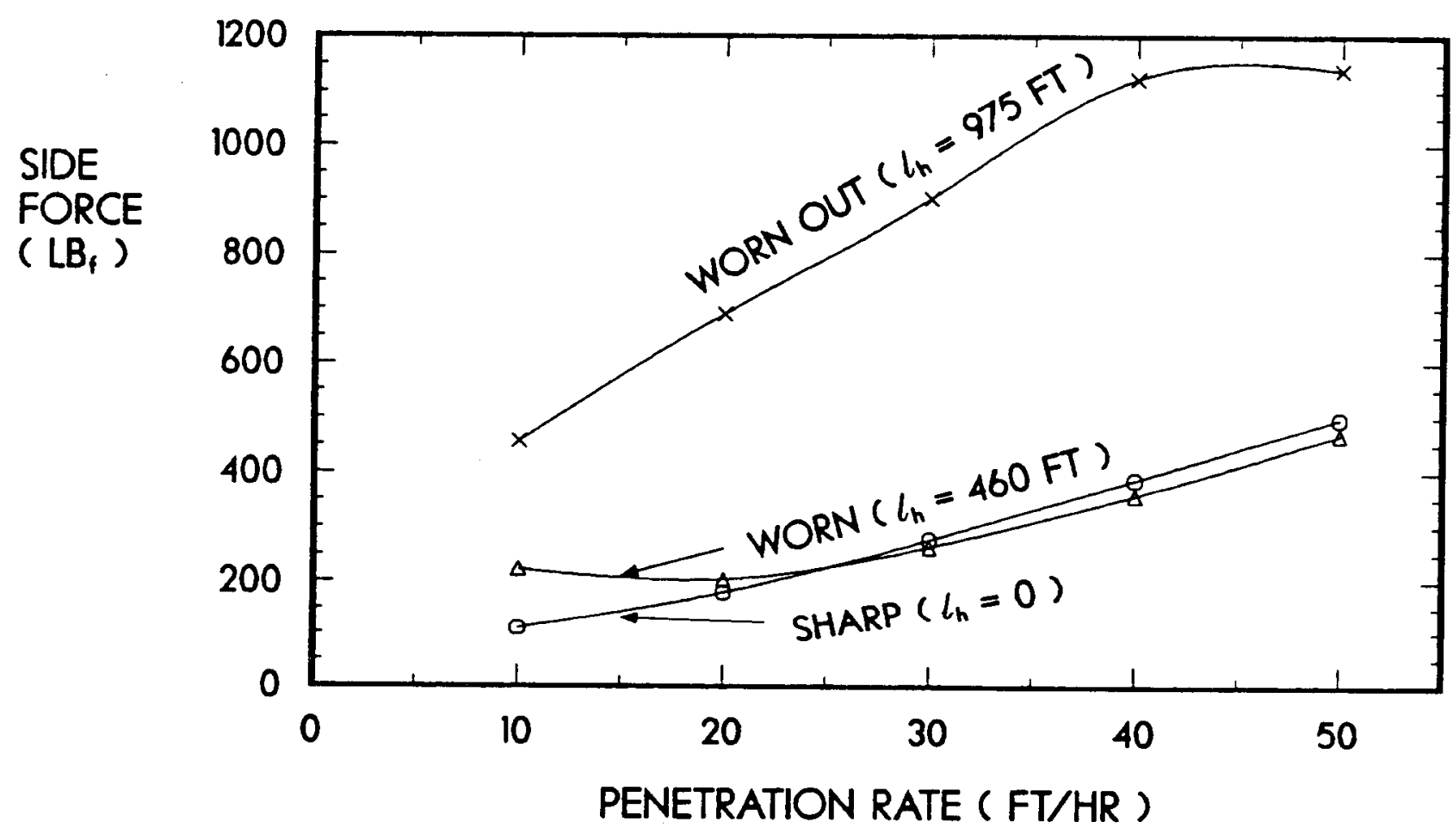

Figure 39 - Predicted resultant bit side force at specified penetration rates in the demonstration analysis. 


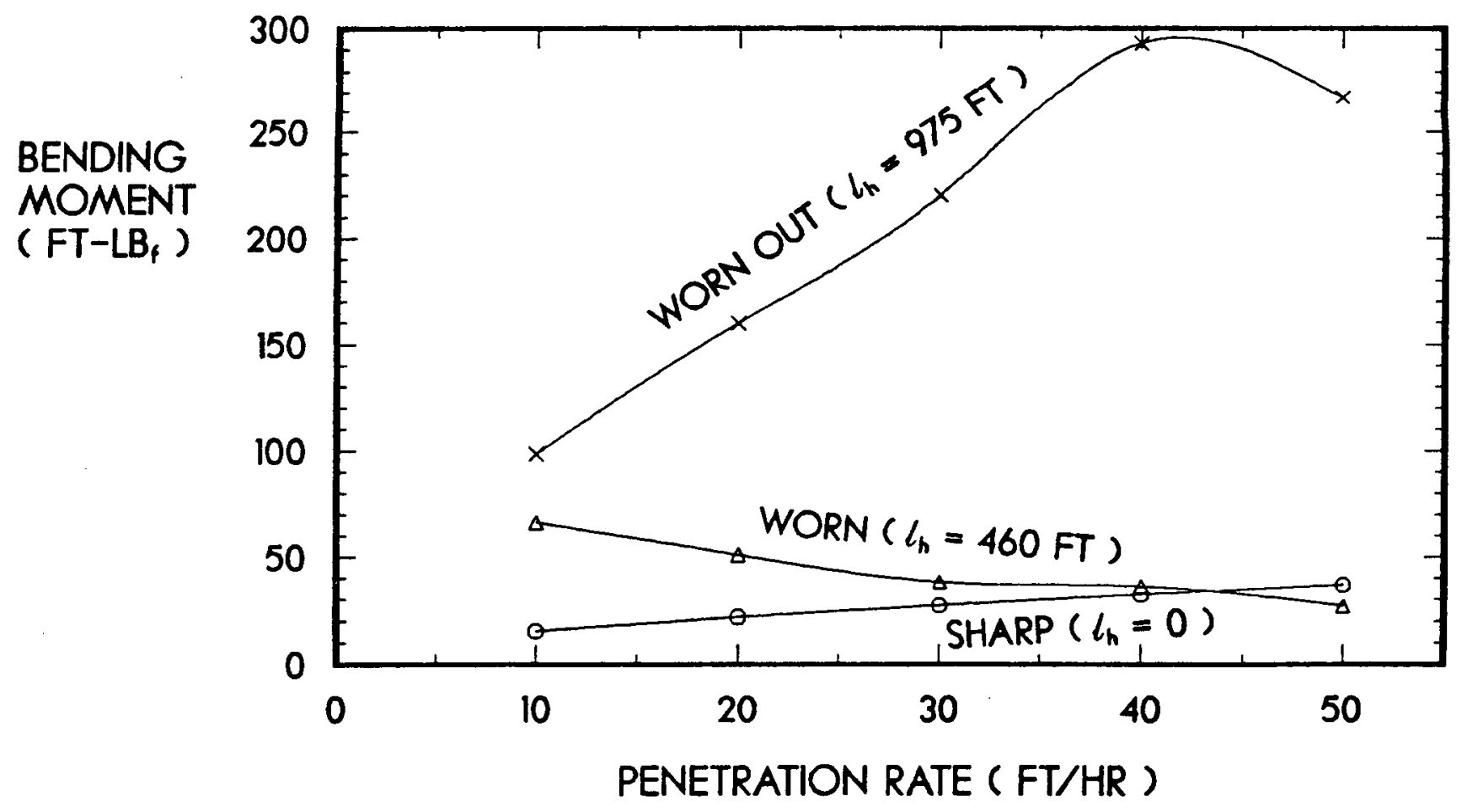

Figure 40 - Predicted resultant bit bending moment at specified penetration rates in the demonstration analysis. 
by increased cutter interaction resulting from the closer radial spacing of cutters at increased radial positions on the bit. As the cutters wear, however, the overriding effects of wearflat area cause the penetrating force distribution to change as shown.

The wear ratios resulting from the computed penetrating forces are shown in Figure 35. The integrated effects of the wear ratios at all stages of wear are responsible for the wearflat areas shown in Figure 33.

The computed wearflat temperatures for the baseline analysis are shown in Figure 36. These results indicate that, even when the cutters are severely worn, temperatures are below $350^{\circ} \mathrm{C}$ and thermally-accelerated wear should not occur. It should be noted, however, that the friction coefficient assumed for this analysis was only 0.07 , and the cooling fluid temperature was assumed to be only $80^{\circ} \mathrm{F}$. If either of these quantities were higher, which is likely under actual drilling conditions, cutter wearflat temperatures could exceed the critical level, and accelerated wear would occur. Continuing the analysis beyond the designated "worn out" stage indicated that cutter 16 reached the $350^{\circ} \mathrm{C}$ limit after about $1270 \mathrm{ft}$ of drilling at a penetration rate of $30 \mathrm{ft} / \mathrm{hr}$.

The computed WOB is plotted as a function of ROP in Figure 37 . We see a large effect of cutter wear on bit performance. Although the bit requires only $3500 \mathrm{lbf}$ WOB to drill at $30 \mathrm{ft} / \mathrm{hr}$ when sharp, it requires nearly ten times that weight to maintain the same ROP when the bit is worn out.

Perhaps an even more compelling indication that the bit is worn out is the excessive drilling torque predicted for this wear condition. As shown in Figure 38, torque increases with cutter wear to levels that conventional drilling practices would not tolerate, resulting in the bit being pulled.

The resultant bit side force predicted for the baseline analysis is shown in Figure 39. This force would develop if the bit were constrained by the walls of the borehole to follow a straight line and to rotate concentrically about its geometric center. In reality, a bit side force would tend to make the bit cut deeper on the side opposite the force, resulting in an oversize hole and eccentric rotation of the bit, or bit wobble. Such imbalance in the bit design is usually undesirable. When worn, the side forces are less than 38 of the WOB. This would seem to suggest that the bit is not greatly unbalanced in the worn condition, but it is not known what levels of side force are significant, especially under dynamic conditions.

Similar comments can be made with respect to the predicted bending moments, shown in Figure 40. (Note that the plotted data are the resultant bending moment, determined by taking the square of the sum of the squares of the bending moments about the $x^{\prime}$ - and $y^{\prime}$-axes.) In the case of bending moment, the drill pipe is capable of providing a reactive torque to prevent excessive tilting of the bit. Again, however, the effects of the bending moment on the dynamic response of the drill string are not known but could be significant. 


\subsection{Other Program Features}

In addition to those already discussed, the program also has the following features:

1) The program checks for gaps between cutters where uncut rock could leave the bottomhole pattern such that the bit will not drill. If the program detects such gaps at any point in the wear process, it informs the user and allows the user to terminate the session. Although calculations may continue under such conditions, the results will be uncertain.

2) Since the program allows cutters to wear at different rates, it is possible under some conditions that a cutter will reach the point where it no longer contacts the rock. Under these conditions, the program properly predicts zero cutting forces for such a cutter. As a result, the cutter will not wear during the next specified wear increment. Eventually, the wear of surrounding cutters will "catch up" and the cutter will again contact the rock surface and begin to wear.

3) The program predicts results similar to the "wearing in" process that has been observed in laboratory bit tests; i.e., weight-on-bit is slightly higher when the the cutters are sharp than it is after the cutters have worn slightly. Numerically, this is due to the algorithm that uses the larger of the sharp- or worn-cutter correlation force predictions for a given cutter. When the cutter is slightly worn, the worn-cutter force correlation predicts lower forces that the sharp-cutter correlation, so the sharp cutter correlation is used. For a given penetration rate, however, a slightly worn cutter has a slightly lower effective depth of cut than a sharp cutter. As a result, the cutter forces predicted with a slightly worn cutter at a given penetration rate are lower than those predicted with a sharp cutter at the same penetration rate. of course as the cutters wear further, the worn-cutter force correlation predicts higher forces that the sharp-cutter correlation, and weight-on-bit increases with cutter wear thereafter.

4) Since the program files the new wear configuration for each increment of bit wear, the user can terminate the program at any point and re-start the program by specifying the appropriate file as the initial wear configuration file. It should be noted that re-starting the program sets the cumulative drilled borehole length back to zero. Furthermore, other bit performance predictions will not be precisely the same in a re-start situation as in a continuous run because the number of significant digits stored on disk for the wearflat dimensions is less than that stored in computer memory during a continuous run. In most cases, however, the differences in predicted results are not significant. 


\subsection{PREDICTED EFFECTS OF DESIGN AND OPERATION ON BIT PERFORMANCE}

Now that baseline results have been established for the demonstration analysis, the effects of several important design and operating variables can be assessed. These effects were determined by modifying the bit design and/or operating parameters and repeating the analysis. Results for the modified analyses are illustrated by comparing the performance parameters predicted after drilling the same footage as in the baseline analysis (i.e., 0,460 , and $975 \mathrm{ft}$ ).

\subsection{Effects of Bit Profile}

Perhaps the most obvious of the bit design variables is the bit profile. The profile used in the baseline analysis was relatively flat, with only a 1.0 inch range in cutter longitudinal locations over the 8.5inch diameter bit. To assess the effects of bit profile, an analysis was perfomed using the bullet-nose bit design shown in Figure 41 . The cutter locations are identical to those of the baseline analysis, except that the longitudinal locations range over 4.0 inches instead of 1.0 inch, and there is no concave portion in the center of the bit.

Shown in Figure 42 are the predicted wearflat areas for the bullet-nose bit. The primary effect of bit profile in this example is to increase wear on most of the cutters, especially those near the nose of the bit (cutters 1-9). This result agrees with field experience, which generally shows that flatter bit profiles are necessary when drilling hard rock, where uneven cutter wear is usually a problem.

Although the bullet-nose bit wears faster, the computed results indicate that in the early stages of drilling, the WOB is lower than that of the baseline analysis, as shown in Figure 43. When plotted in this manner, it is seen that for a given WOB, the bullet-nose bit drills at a much higher ROP than the baseline bit after both have drilled $460 \mathrm{ft}$ of hole. Since the bullet-nose bit wears faster, however, the effects of wearflat area eventually become dominant. After $975 \mathrm{ft}$ of drilling, the bullet-nose bit has become so worn that it drills much slower than the baseline bit design at the same WOB.

The predicted drilling torque for the bullet-nose bit was higher than that for the baseline analysis at all drilled borehole lengths. In the sharp condition, the differences ranged from 8 to 258 , depending on ROP. After $460 \mathrm{ft}$ of drilling, the bullet-nose bit torque was 12 to $17 \%$ higher, and after $975 \mathrm{ft}$, it was 8 to 258 higher. Side forces and bending moments were also more excessive with the bullet-nose bit. This indicates that the sharper the bit profile, the more carefully cutters must be placed in order to balance the bit design.

\subsection{Effects of Cutter Placement Density}

In this analysis. the number of cutters was reduced from 21 to 15 in order to assess the effects of using a lower cutter placement density. Cutters $8,10,12,14,16$, and 18 were removed from the baseline design, with other cutters remaining in their original positions. The cutters that 


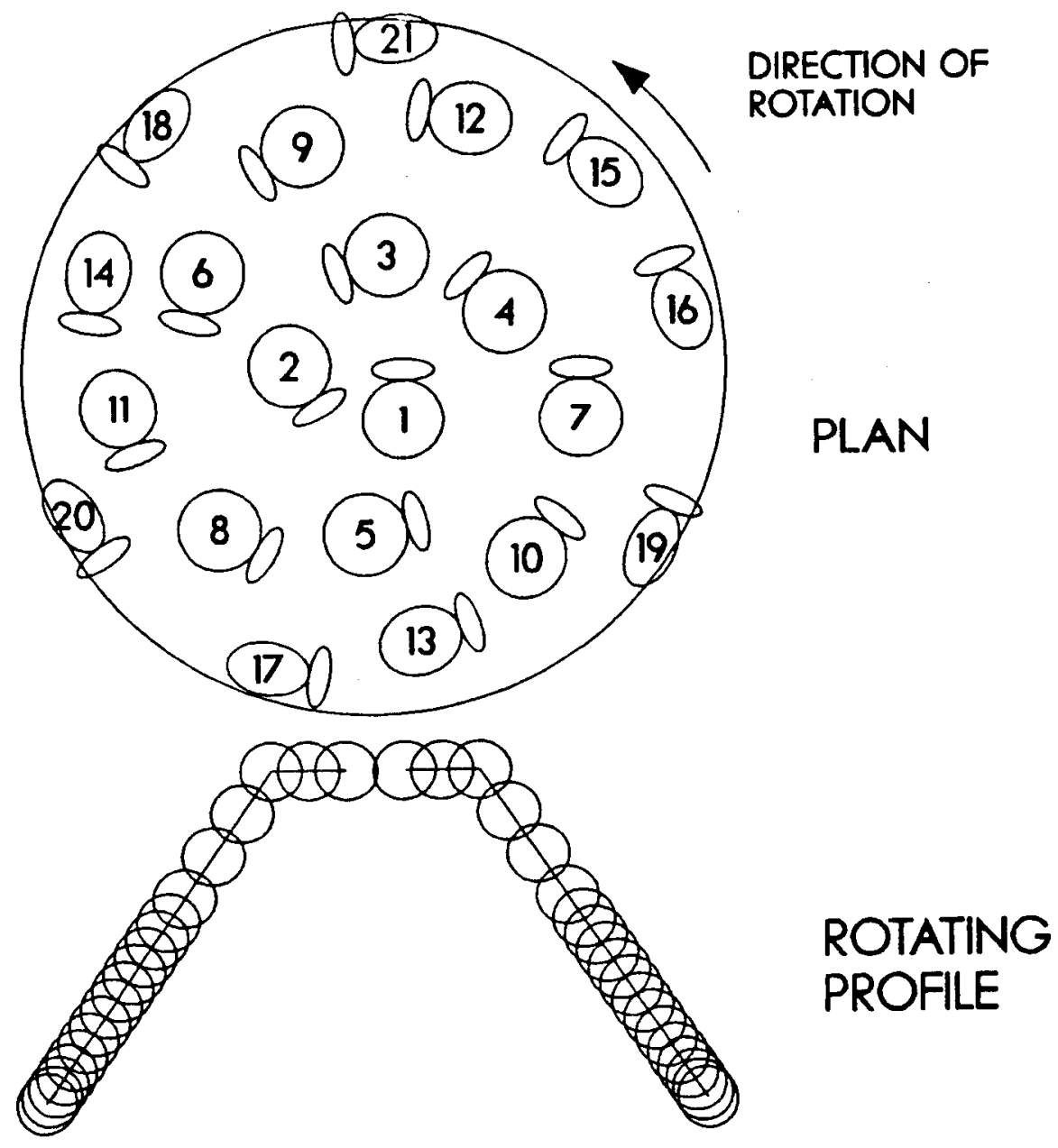

Figure 41 - Schematic of bullet-nose bit design used in modified analysis to illustrate the effects of bit profile. 


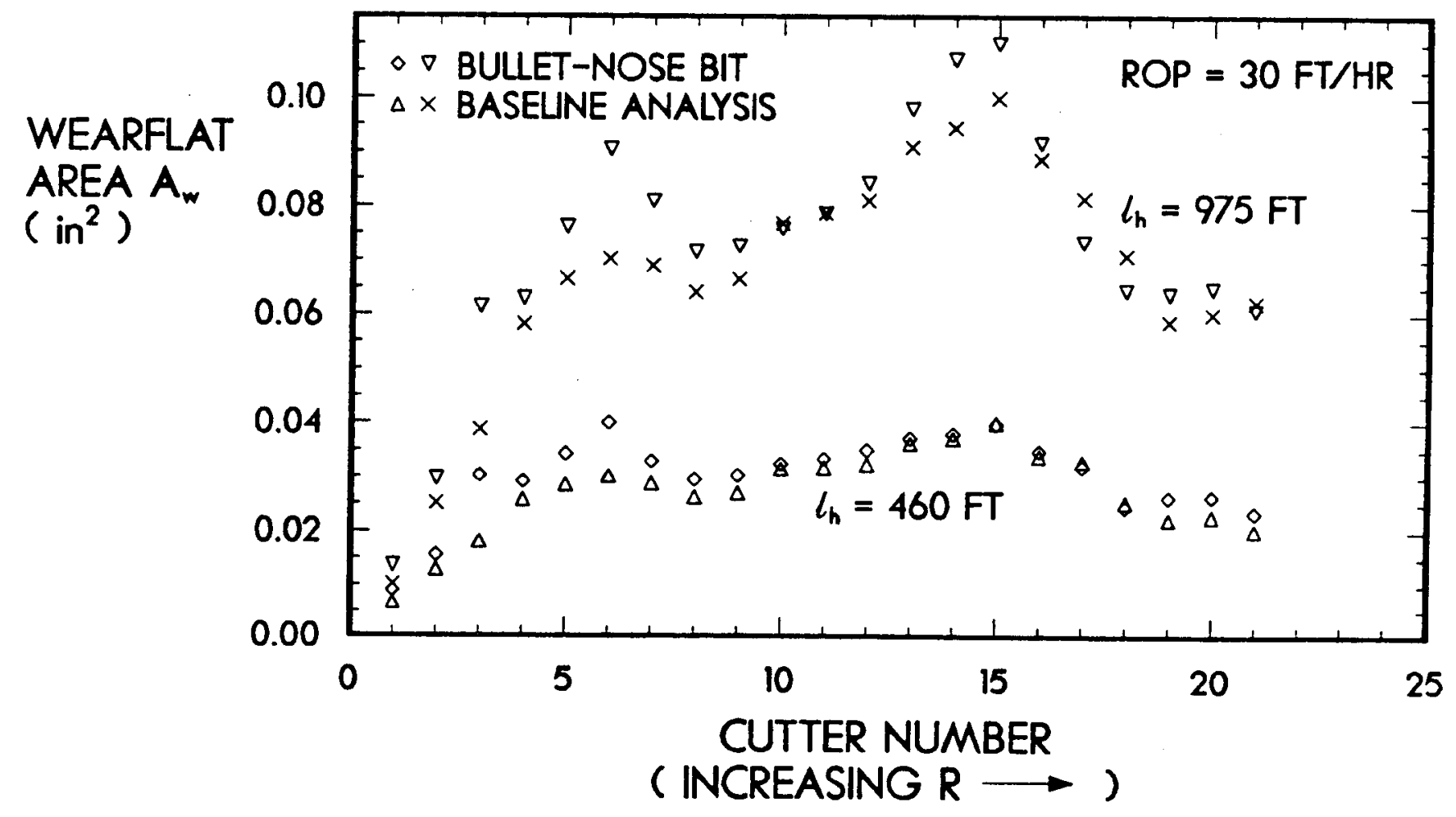

Figure 42 - Predicted cutter wear distribution across bit, showing the effects of bit profile. 


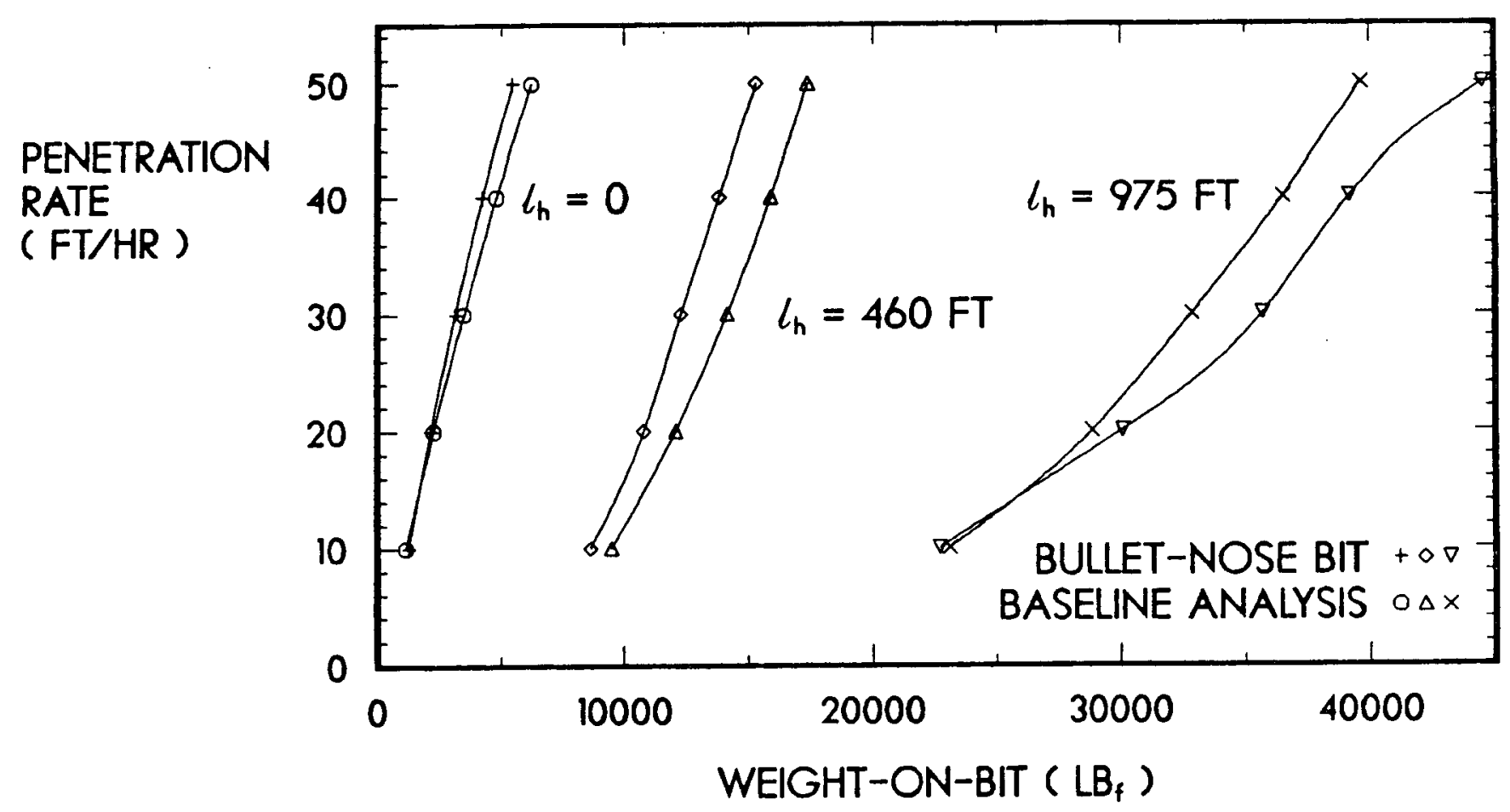

Figure 43 - Specified penetration rate plotted as a function of predicted WOB to illustrate the effects of bit profile. 
were removed were so chosen in an effort to maintain balance in the design. The resulting 15-cutter bit design is shown in Figure 44.

In this case, the remaining cutters in the reduced density region (cutters 7-19) experienced greatly increased wear, as shown in Figure 45 . This is caused by the reduced interaction and, consequently, higher penetrating forces on these cutters as compared with the baseline analysis. The predicted wearflat cutter temperatures at a ROP of $30 \mathrm{ft} / \mathrm{hr}$ indicate that cutter 15 exceeds $350^{\circ} \mathrm{C}$ after a hole length of $880 \mathrm{ft}$; thus the cutter15 wearflat area would actually be even larger than that shown in Figure 45 at $975 \mathrm{ft}$.

Although the number of cutters used in the two bits is significantly different, the difference in required WOB is relatively small. When the bits are sharp, the 15-cutter bit requires 4 to 78 more WOB. After 475 ft of hole, the 15-cutter WOB is lower by 4 to 88 . By the time both bits have drilled $975 \mathrm{ft}$, the 15 -cutter WOB is 3 to 118 lower. Of course, if the accelerated wear of the cutters that experience thermal wear had been taken into account, the larger wearflat areas for those cutters would have increased the WOB significantly with further drilling after $880 \mathrm{ft}$. Furthermore, since wear rates increase by 1 to 2 orders of magnitude during this mode of wear, the affected cutters can, for all practical purposes, be considered destroyed. When this happens, the bit may be left in a configuration that will not drill.

These results suggest two ways for defining bit life that may be inherently related under certain conditions. The first is the drilled footage at which thermally-accelerated wear begins on any cutter. In this case, the life of the 15 -cutter bit would be $880 \mathrm{ft}$, and that of the baseline bit would be $1270 \mathrm{ft}$ (at a penetration rate of $30 \mathrm{ft} / \mathrm{hr}$ ).

Alternatively, we may define the bit life as the drilled footage at which a performance parameter, such as WOB, drilling torque, or bit imbalance, reaches some selected level. In the baseline analysis, we arbitrarily selected a WOB of $40,000 \mathrm{lbf}$ (at $50 \mathrm{ft} / \mathrm{hr}$ ) as a limit on bit life, based on practical levels for this size bit. This resulted in the WOB-bit life criterion of $33,000 \mathrm{lbf}$ at $30 \mathrm{ft} / \mathrm{hr}$ in the baseline analysis. If thermally-accelerated wear occurs, as with the 15-cutter bit, the resulting rapid growth in WOB would cause the bit to more rapidly reach the WOB limit defining bit life. Thus, if the temperature-bit life criterion is reached, it is probable that any practical WOB-bit life criterion will be reached soon thereafter.

In addition to changing the number of cutters, placement density can be modified locally by moving cutters radially and circumferentially. The radial placement of cutters is one of the most important parameters in bit design. To achieve more uniform wear, cutters can be shifted radially to provide a higher placement density in regions of excessive wear and a lower density in regions of low wear.

The circumferential placement of cutters is also important because it affects bit balance during drilling. Excessive side forces can be reduced by shifting cutters circumferentially in the proper directions. 


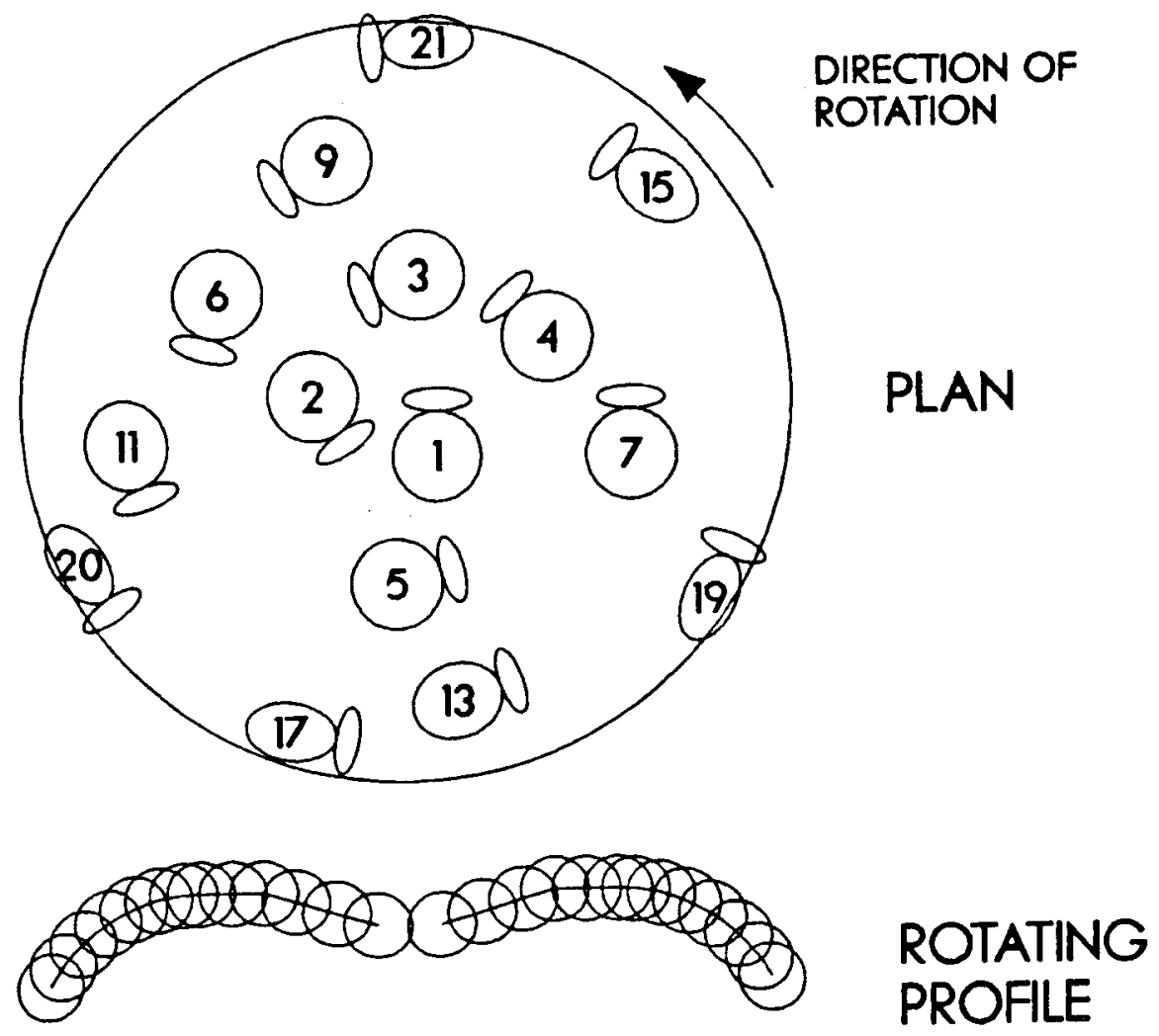

Figure 44 - Schematic of bit design used in modified analysis to illustrate the effects of cutter placement density. 


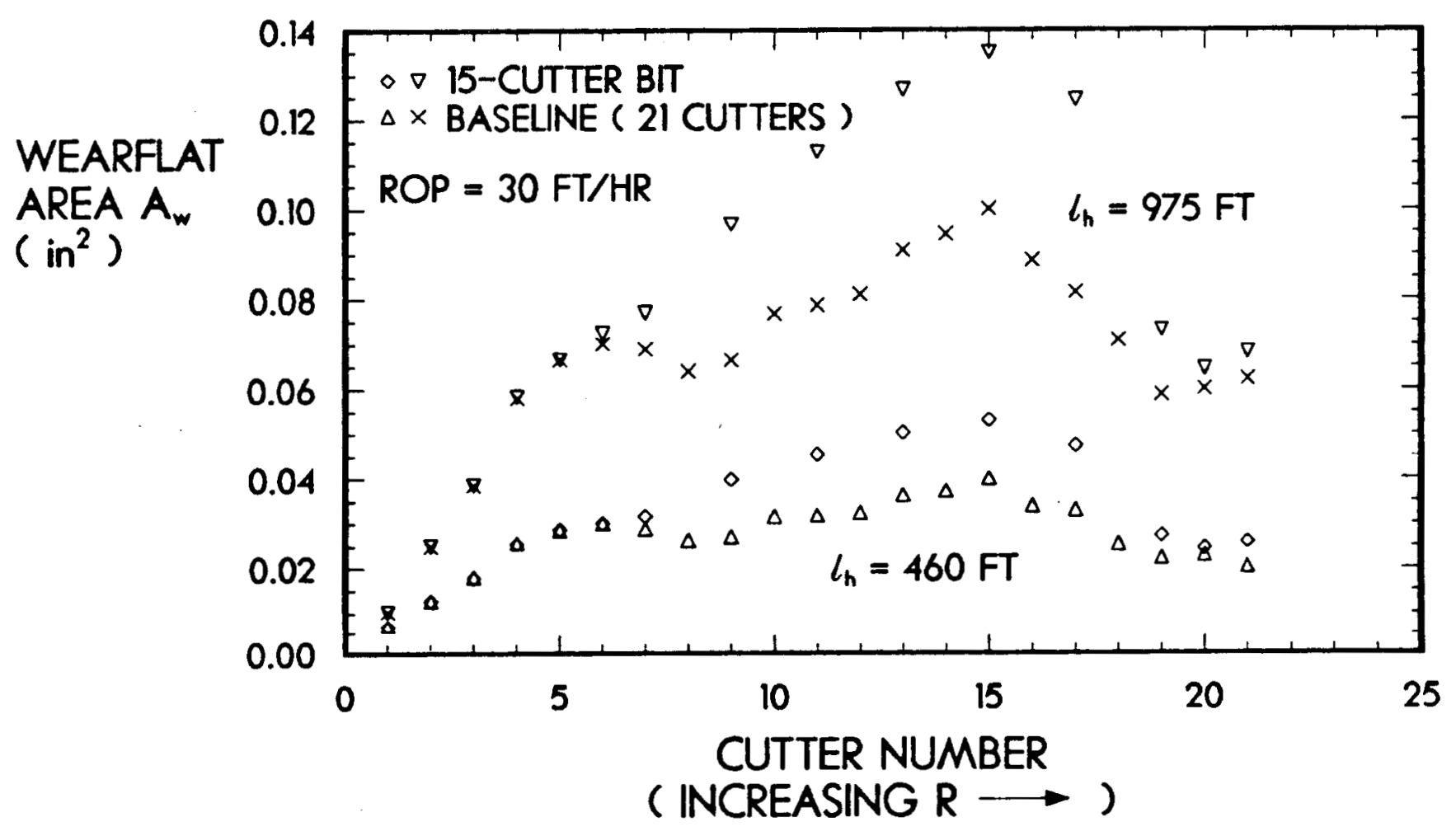

Figure 45 - Predicted cutter wear distribution across bit, showing the effects of cutter placement density. 
Circumferential placement also affects individual cutter forces because it determines how the feed rate of the bit is distributed among the cutters (see Eq. 13d).

\subsection{Effects of Bit Rotary Speed}

For a given penetration rate, the feed rate of the bit (penetration/revolution) decreases as the bit rotary speed is increased (see Eq. 13e). This implies that for a given wear configuration, a selected ROP can be achieved with a lower WOB by increasing rotary speed. To investigate this effect, an analysis was performed by changing the rotary speed in the baseline analysis from 100 to 200 RPM.

The results indicate that when the bit is sharp, the higher rotary speed reduces the WOB requirements by 42 to 548 . Similar reductions in drilling torque were predicted at the higher speed. The lower bit feed rate, however, causes each cutter to travel twice as far in drilling the same length of hole. As a result, the cutters wear faster with respect to footage drilled. This effect is illustrated in Figure 46. Note that all cutters experience increased wear at the higher rotary speed. The wear on cutters 11-18 after $975 \mathrm{ft}$ of hole would be even greater than that shown, because the predicted wearflat temperatures for those cutters exceed $350^{\circ} \mathrm{C}$ after only 650 to $900 \mathrm{ft}$.

As a result of the computed wear pattern, the wOB for the higher rotary speed is 11 to 188 higher after $465 \mathrm{ft}$ and 25 to 268 higher after $975 \mathrm{ft}$ of hole. Similar increases in drilling torque were predicted for the higher rotary speed. The conclusion we reach is that, even under conditions where thermal wear effects are not important (e.g. at $460 \mathrm{ft}$ ), increased rotary speed is detrimental to bit life and, consequently, to the penetration performance of the bit. This agrees with results obtained in our earlier work [31]. Under conditions where a higher ROP is required, it is better to achieve it by increasing WOB rather than rotary speed.

\subsection{Effects of Waterjet Assistance}

In the baseline analysis, cutters $13-16$ experienced greater wear than the other cutters. In the present modified analysis, the beneficial effects of waterjet assistance are demonstrated by simulating placement of jets in front of those cutters. For these cutters, the single cutter correlation constants measured in the tests with 4500 psi waterjets (Figure 16) were used. This was done by specifying those cutters to be of type $B$ and entering the proper correlation constants into the OPCOND.DAT data file.

Shown in Figure 47 are the computed wear distributions. Waterjet assistance is seen to reduce wear by more than one-half on the assisted cutters. Furthermore, since these cutters do not wear as fast as the adjacent cutters, they remove more rock than they would otherwise remove after a given hole length. As a result, the waterjet-assisted cutters interact more with adjacent cutters, thereby reducing wear on the adjacent cutters as well. This effect is noticeable as far in toward the center of the bit as cutter 3 . 


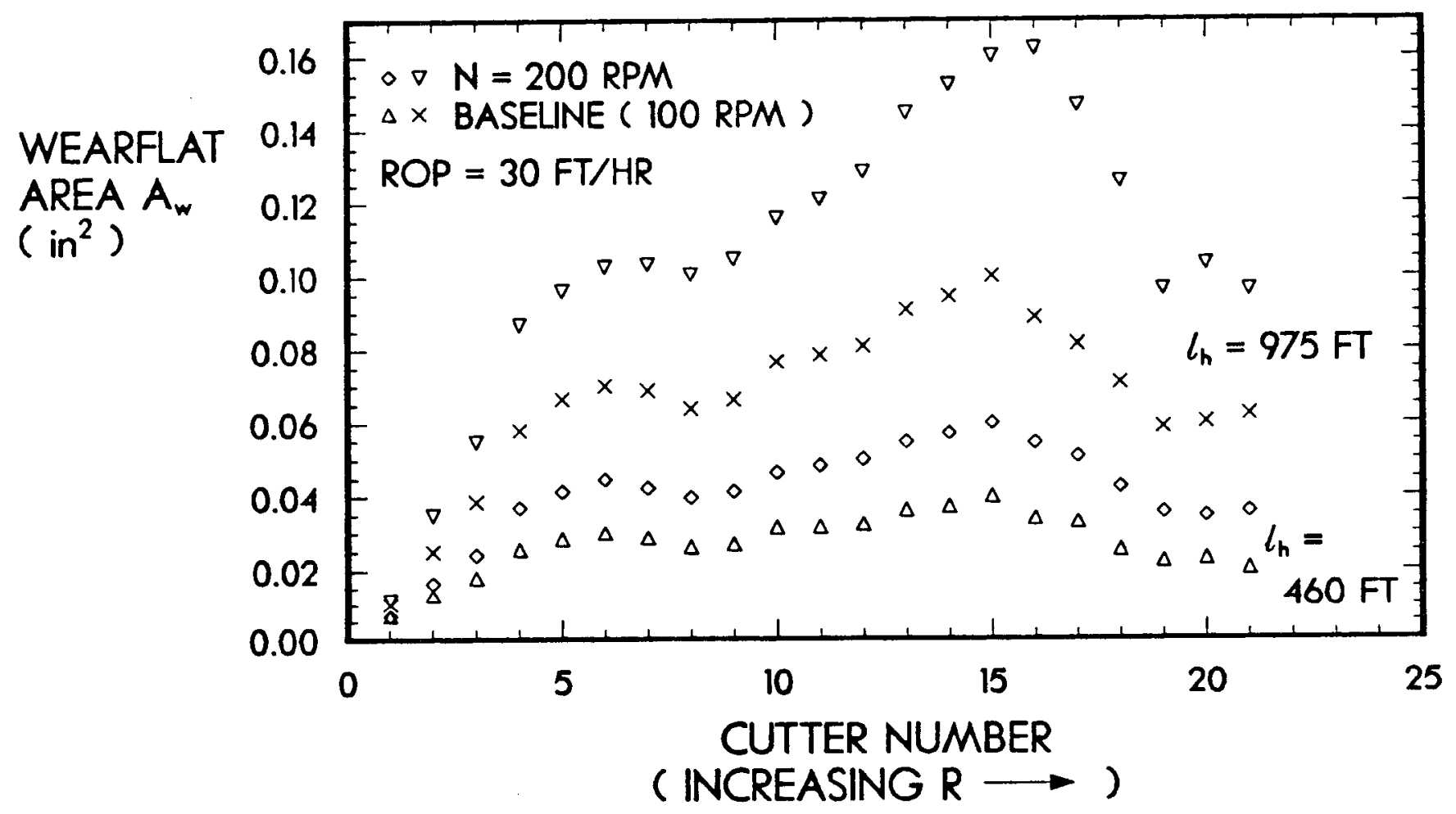

Figure 46 - Predicted cutter wear distribution across bit, showing the
effects of rotary speed. 


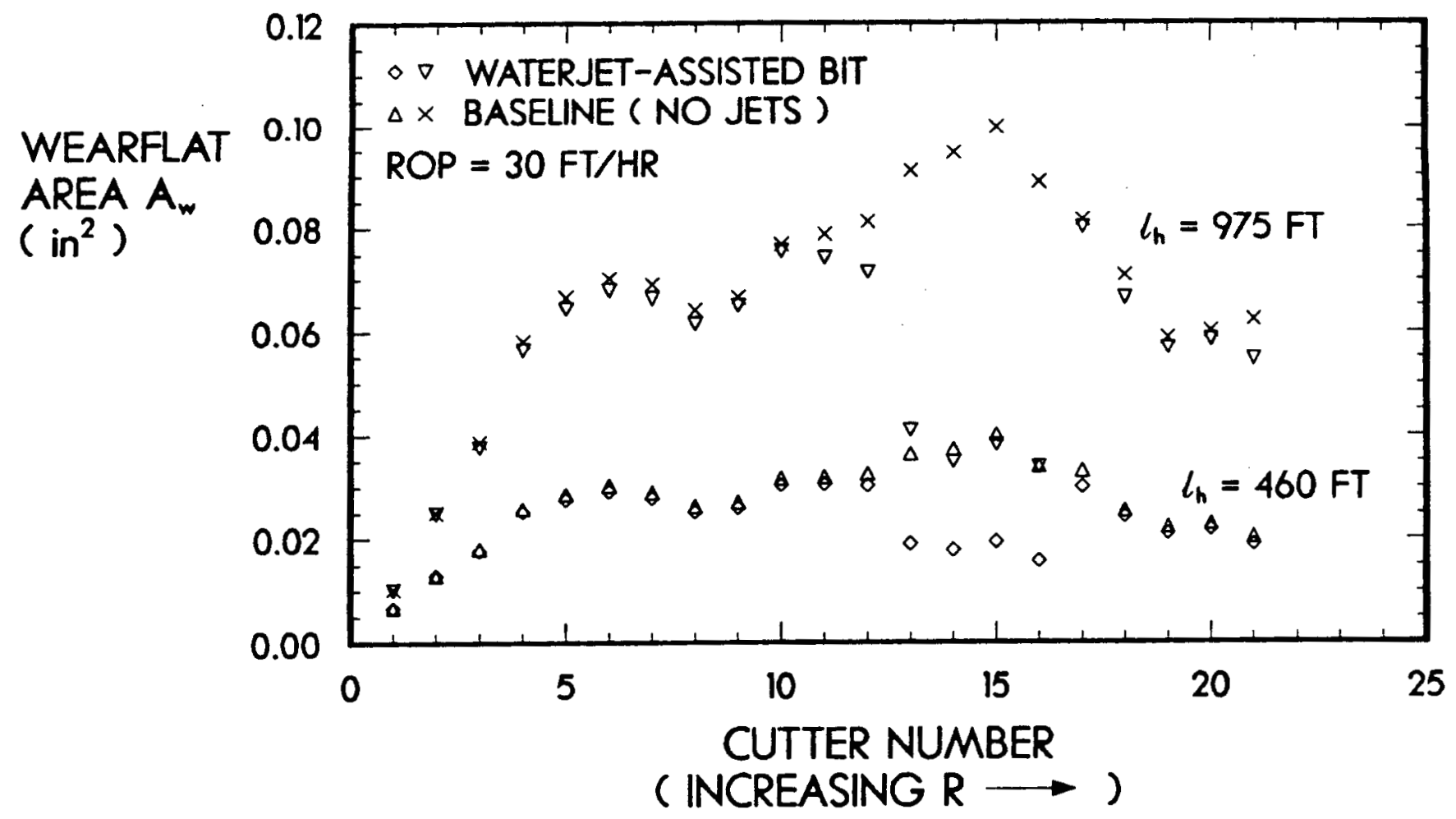
Figure 47 - Predicted cutter wear distribution across bit, showing the
effects of waterjet assistance for selected cutters $(13-16)$. 
The reduced wear on the bit is responsible for a significant improvement in drilling performance. The WOB required with the jet-assisted bit was computed to be 19 to 238 lower after $460 \mathrm{ft}$ of hole and 23 to $27 \%$ lower after $975 \mathrm{ft}$. Even greater reductions in drilling torque were predicted for the jet-assisted bit: 27 to $33 \%$ after $460 \mathrm{ft}$ and 29 to $36 \%$ after $975 \mathrm{ft}$ of drilling. If WOB is used as the criterion for declaring the bit worn out, the jet-assisted bit is predicted to have a bit life of 1225 $\mathrm{ft}$, as compared with $975 \mathrm{ft}$ in the baseline analysis. If the onset of thermally-accelerated wear is used as the bit life criterion, the jetassisted bit drills for 2950 ft, compared with $1220 \mathrm{ft}$ in the baseline analysis.

\subsection{Effects of Wear Mode}

In this analysis, the soft-rock wear mode was specified to demonstrate the effects on wear patterns and bit performance. Results are shown in Figure 48. After drilling $460 \mathrm{ft}$, the cutter wearflat areas are up to $30 \%$ smaller than those predicted for the hard-rock wear mode assumed in the baseline analysis. Beyond this footage, the difference in wear becomes even more pronounced. After the bit has drilled $975 \mathrm{ft}$, the wearflat areas in the soft-rock wear mode are up to 638 smaller than those predicted for the hard-rock wear mode.

In the hard-rock wear mode, the wearflat area in contact with the rock is a strong function of the volumetric cutter wear, which in turn is proportional to the cutter penetrating force. Since the wearflat area has a large effect on penetrating force, wear in the hard-rock wear mode tends to occur at an accelerating rate with respect to footage drilled, even in the absence of thermal effects.

The wearflat area in the soft-rock wear mode, however, is not as sensitive to wear volume. In other words, a given increase in $\mathrm{dV} / \mathrm{d} l_{\mathrm{h}}$ does not produce as large an increase in $A_{w}$ as it does in the hard-rock wear mode. As a result, the wearflat area growth rate actually decreases as drilling continues. In this analysis, the maximum predicted wearflat area growth between hole lengths of 460 and $975 \mathrm{ft}$ is only about 348. This compares with 1508 growth in maximum wearflat area in the case of the hardrock wear mode over the same footage.

The performance parameters in the soft-rock wear mode behave in a similar fashion. The WOB requirements after $460 \mathrm{ft}$ of hole are within $16 \%$ of those predicted for the hard-rock wear mode. By the time both bits have drilled $975 \mathrm{ft}$, however, the bit worn in the soft-rock mode requires 50 to 518 less WOB than that in the hard-rock mode. Similar results were obtained with respect to drilling torque.

Because of the modest wearflat growth, the cutter temperatures in this analysis with the soft-rock wear mode never approach the critical $350^{\circ} \mathrm{C}$ limit before the cutters are worn to the center of the compacts. When the wear reached this point in the analysis, at 18,666 ft, the calculations were terminated, and the bit was considered worn out. In terms of the WOB-bit life criterion of 33,000 lbf at $30 \mathrm{ft} / \mathrm{hr}$, the bit was predicted to drill 


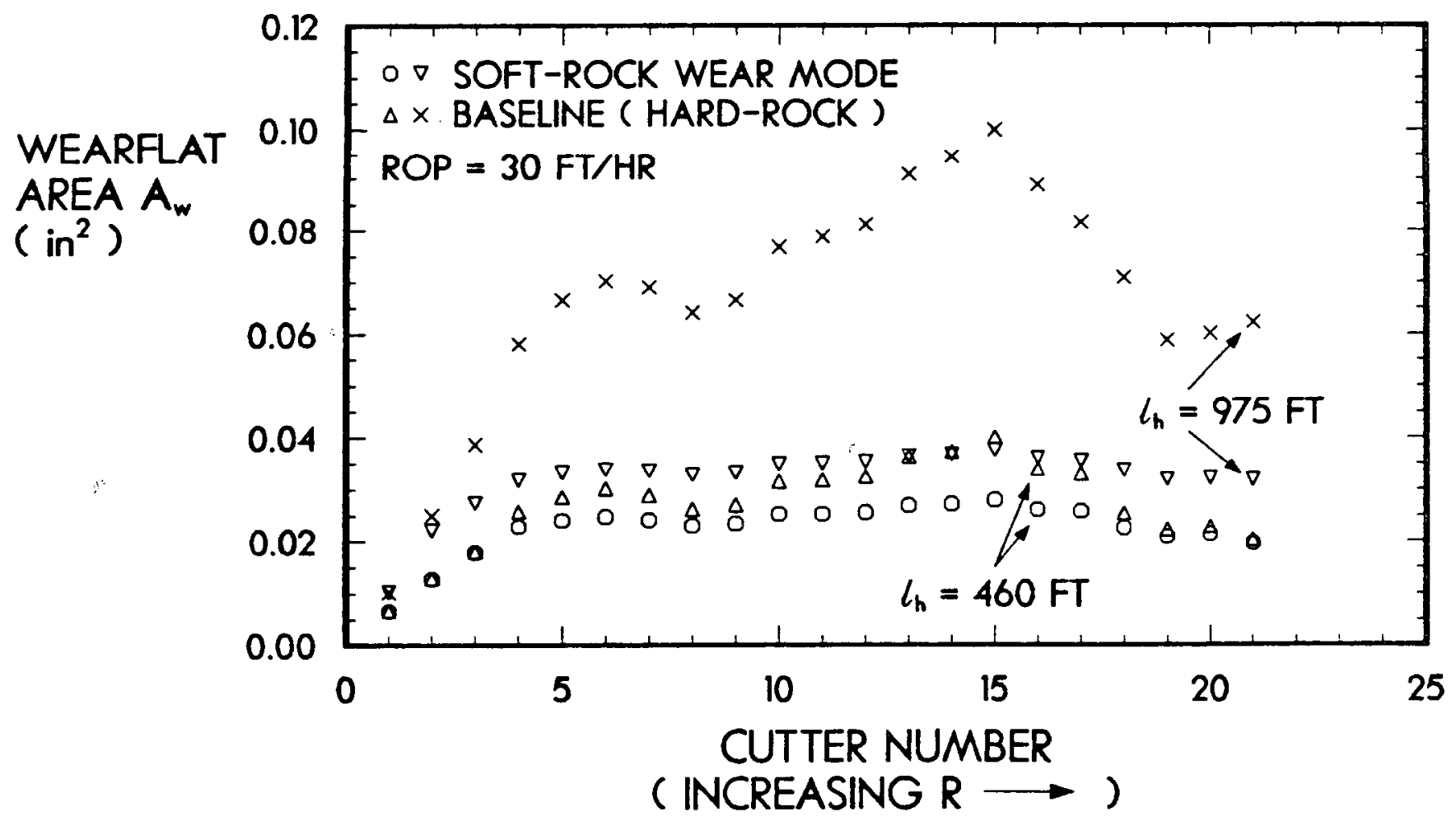
Figure 48 - Predicted cutter wear distribution across bit, showing the
effects of wear mode. 
over $11,500 \mathrm{ft}$. It is therefore concluded that significantly greater bit life can be attained when a bit wears in the soft-rock wear mode rather than in the hard-rock wear mode.

\subsection{Discussion}

The computer code developed in this study predicts that the baseline bit design will drill relatively hard rock at a rate of $10-50 \mathrm{ft} / \mathrm{hr}$ and will last for approximately $1000 \mathrm{ft}$ with a WOB of less than $40,000 \mathrm{lbf}$ and a drilling torque of less than $6000 \mathrm{ft}-1 \mathrm{bf}$. These and other predicted results appear quite reasonable and are comparable to performance typically achieved with this size bit.

For example, in geothermal drilling tests in the Imperial Valley, California, an 8-3/4" PDC bit drilled $555 \mathrm{ft}$ at an average ROP of $55 \mathrm{ft} / \mathrm{hr}$, with an estimated life of about $1000 \mathrm{ft}$ [17]. The drilled interval contained sandstone, shale, siltstone, and igneous formations. The unconfined compressive strength of the sandstone in this interval was 11,000 psi, about one-half of the Sierra White granite assumed in the present analysis. Under the elevated downhole stresses present in the field test, however, the strength of the sandstone may be comparable to that of the granite at atmospheric pressures. Furthermore, the abrasive wear constant assumed in the demonstration analysis is probably close to the abrasive wear constant of the sandstones drilled in the field tests.

The thermal conditions assumed in the analysis were relatively mild. Downhole cooling fluid temperatures typically exceed $125^{\circ} \mathrm{F}$ in petroleum drilling and $200^{\circ} \mathrm{F}$ in geothermal drilling, well above the $80^{\circ} \mathrm{F}$ assumed in the analysis. Friction coefficients of 0.03-0.20 in water and $0.10-0.32$ in air have been measured between PDC cutters and rock. It is therefore possible for the cutter wearflat temperatures to exceed those predicted in the baseline analysis, according to Eq. 31. In that case, thermal wear effects could reduce bit life well below the $1000 \mathrm{ft}$ predicted here.

It has been shown that bit profile can significantly affect bit performance. This is due to the fact that the profile not only affects cutter density and thus interaction, but it also controls the orientation of the cutter penetrating forces. Forces oriented at steep angles, such as those on the side of the bullet-nose bit, contribute directly to cutter wear and bit side forces but have less relative impact on wOB. This explains how a bit design can drill with lower wOB but still experience more rapid wear than a different design.

Nozzles such as those used in the experimental part of this work would produce jets that flow at rates on the order of $12-18$ gal/min with nozzle pressure drops of 2000-4500 psi. Since total drilling fluid flow rates of approximately $300 \mathrm{gal} / \mathrm{min}$ are typical for this size bit, it appears that up to 16-25 nozzles could be utilized on a single bit to assist PDC cutters. The resulting reduction in cutter forces could extend the applicability of PDC bits to much harder formations and more severe operating conditions. Limitations imposed by space requirements and the tendency for nozzles to plug would need to be overcome, but the potential benefits make the concept attractive. 
Bit life in the soft-rock wear mode has been shown to be much greater than that in the hard-rock wear mode. This finding could explain the wide range in bit wear that is sometimes found in formations with apparently similar strengths. If the formation is fractured or has a high percentage of quartz, the probability that the cutters will wear in the hard-rock wear mode is increased, regardless of the apparent strength of the rock. The fact that some PDC bits have experienced bit life as long as 20,000 ft [49], however, is evidence that the soft-rock wear mode is operative under certain conditions.

It should be noted that many of the complex phenomena that contribute to PDC bit wear and performance are by no means completely understood. In this paper, we have developed and used simple models to describe various mechanisms so that the approach can be easily modified as more complete understanding becomes available. PDCWEAR has been written so that different single-cutter force correlations than those developed in this work can be easily substituted if the user so desires.

More work needs to be done to improve understanding of PDC cutting mechanisms. For example, tests should be conducted under elevated fluid and rock stresses to determine how downhole conditions affect these mechanisms. In particular, the effects of ambient pressure on the benefits of waterjet assistance should be investigated.

Finally, the approach developed in this report for predicting PDC bit performance and wear needs to be tested. This could be done by comparing predicted bit performance parameters, such a WOB and drilling torque, with results measured in full-scale PDC bit tests under carefully controlled atmospheric and elevated pressure conditions. 


\subsection{CONCLUSIONS}

1) The penetrating force imposed on a worn PDC cutter at a given depth of cut is approximately proportional to the wearflat area in contact with the rock.

2) Within the limited range of PDC cutter compact sizes tested (0.5-0.75 inch diameter), the penetrating force is independent of compact diameter. This implies that larger cutters are more efficient in rock removal than smaller ones.

3) The penetrating stress required to cut to a given depth with a PDC cutter can be significantly reduced by directing low- to moderatepressure waterjets onto the rock surface immediately ahead of the cutter. Reductions of 10-158 at 2000 psi nozzle pressure drop and $50-$ 65 reductions at 4500 psi were measured in the present study, which was conducted at atmospheric pressure in granite.

4) Two distinct PDC cutter wear modes occur, depending on the type of rock drilled. Soft, plastic rocks tend to wear the flat at an angle with respect to the rock surface, which keeps a smaller area in contact with the rock and the cutter in a sharper condition. Hard, brittle rocks and conditions under which cutter impact loading is prevalent tend to wear the flat parallel to the rock surface, leading to a larger area in contact with the rock and higher cutting forces.

5) A method has been developed for using single-cutter data to predict cutter forces and bit performance for arbitrary PDC bit designs. This method is incorporated into the computer code PDCWEAR to make it available for general PDC bit design analysis. The code has been shown to produce reasonable predictions of WOB, drilling torque, and bit life.

6) Unless cutter locations are carefully chosen, significant bit side forces and bending moments can develop during drilling. The resulting bit imbalance can cause bit wobble, leading to an overgage hole and possible bit deviation.

7) Bit profile can significantly affect PDC bit performance and wear. It has been shown that bits with sharper profiles tend to initially require less WOB than flat profiles, but sharper profiles wear faster and eventually require greater WOB as drilled borehole length increases.

8) The distribution of cutters on a PDC bit can be used as a means for equalizing wear across the bit face. Cutters in regions of low placement density wear faster than those where placement density is high. Within limits, the WOB and torque required to drill at a specified penetration rate is relatively independent of the number of cutters used on the bit. A bit with fewer cutters will, however, wear faster and eventually require greater WOB as drilled borehole length increases.

9) Increased bit rotary speed is detrimental to bit life, even under conditions where thermal wear effects are not important. Doubling the 
rotary speed from 100 to 200 RPM initially reduces the WOB required to drill at a specified rate, but more rapid wear occurs at the higher speed and eventually leads to greater WOB requirements and lower bit life.

10) Waterjet assistance can significantly increase bit life and reduce wOB and drilling torque requirements. Greater than 208 improvements in bit life and $19-368$ reductions in WOB and drilling torque are predicted for a design utilizing only four nozzles operating at 4500 psi pressure drop. If space limitations and nozzle plugging problems could be resolved, this technique could be used to significantly extend the range of applicability of PDC bits. 


\subsection{NOMENCLATURE}

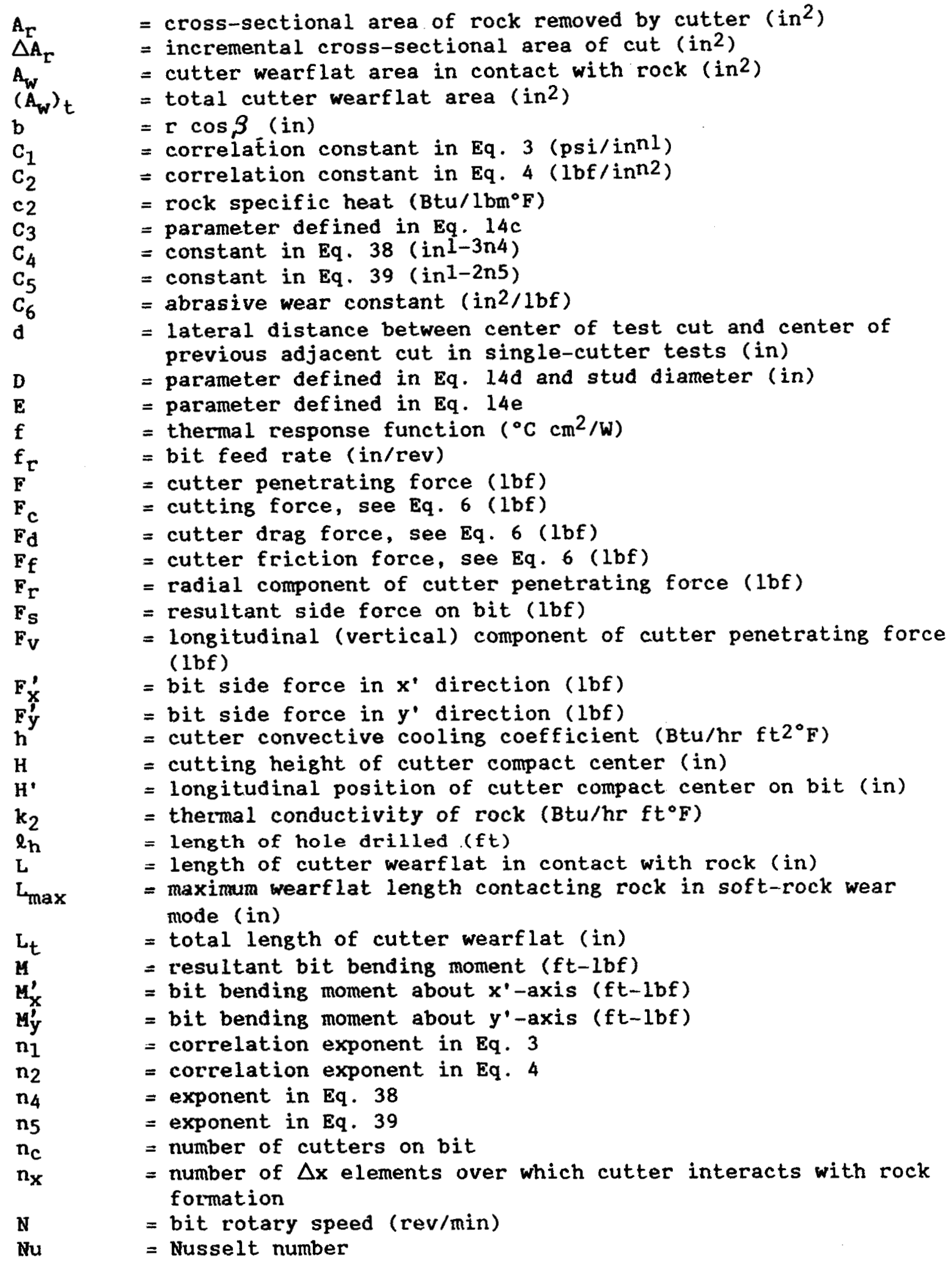




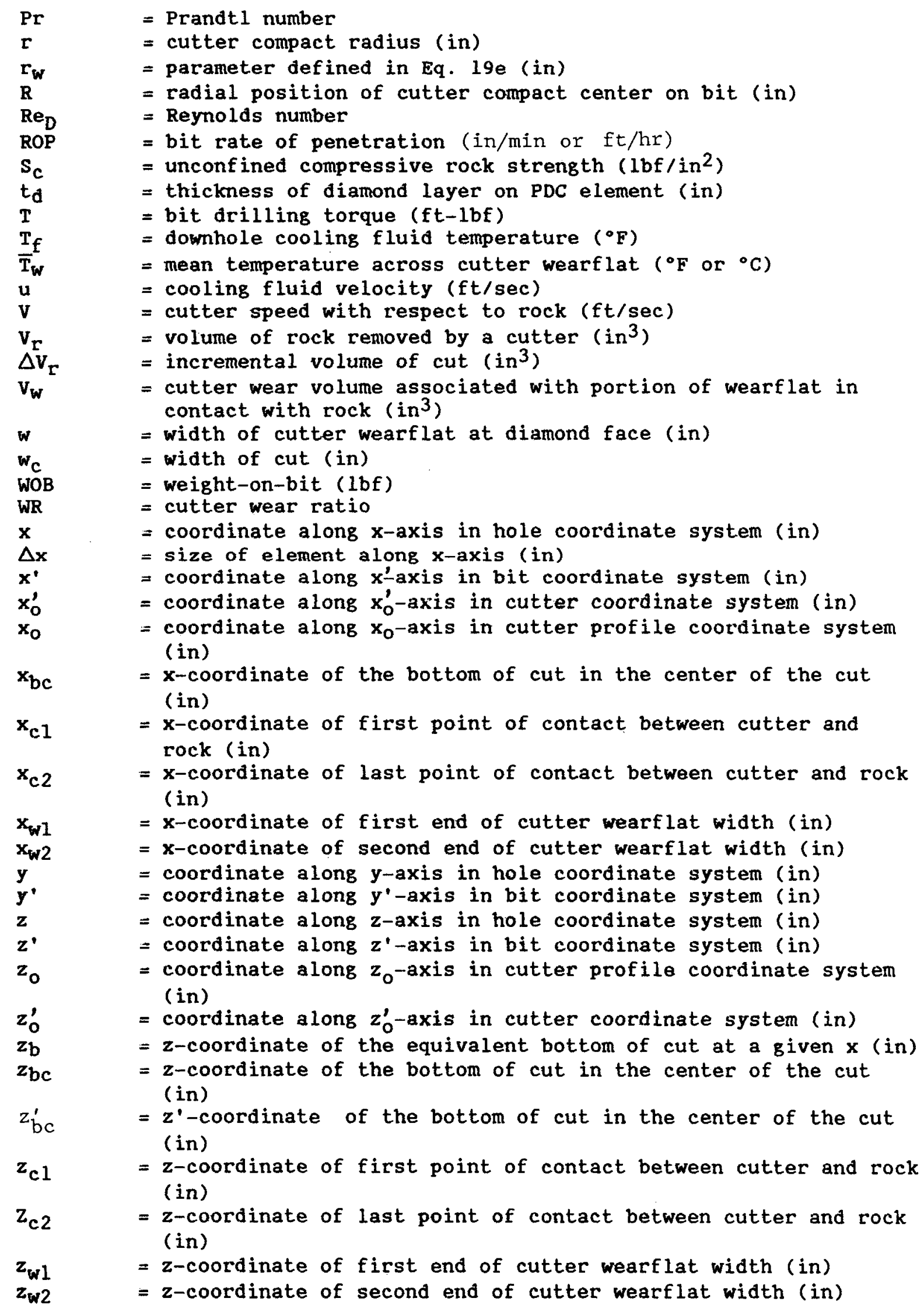



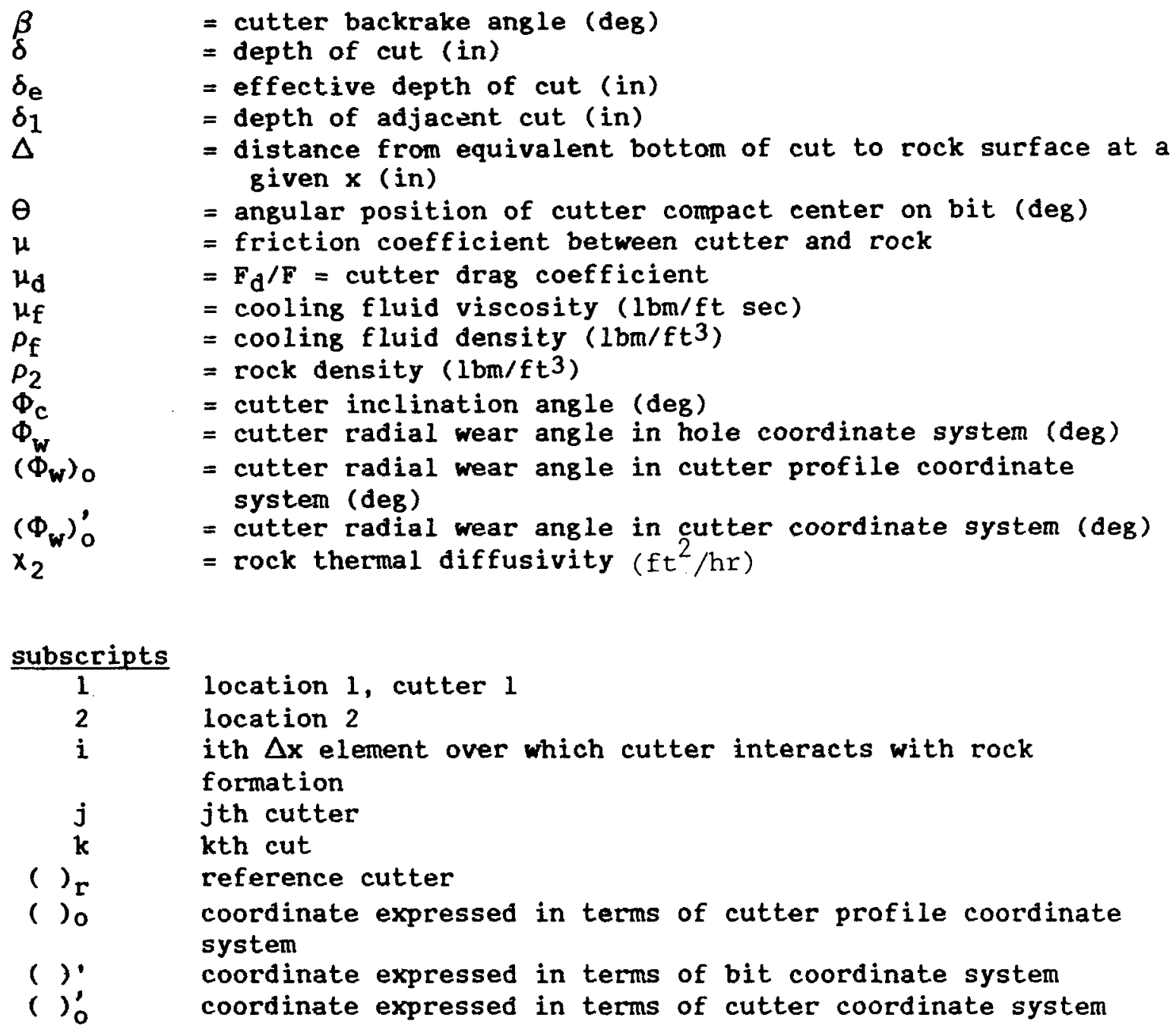

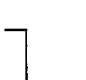


\subsection{ACKNOWLEDGMENT}

This work was supported by the U.S. Department of Energy, Geothermal Technologies Division, at Sandia National Laboratories under Contract DE-AC04-76DP00789.

The assistance of David L. Goodwin with the experimental work is gratefully acknowledged.

$-101-1-102-$ 


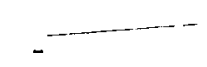

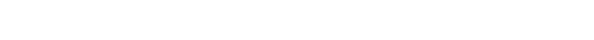
, 


\subsection{REFERENCES}

1. Hendrickson, R.R., Winzenried, R.W., and Jones, A.H.: "High-Temperature Seals and Lubricants for Geothermal Rock Bits: Final Report," Sandia National Laboratories Report SAND81-1404, Albuquerque, NM (Sept. 1981).

2. Hibbs, L.E., Jr., Sogoian, G.C., and Flom, D.G.: "Geothermal Compax Drill Bit Development, Final Technical Report," General Electric Corporate Research and Development, prepared for U.S. Dept. of Energy under Contract DE-AC04-76ET27142 (Apri1 1984).

3. Lin, Y.T.: "The Impact of Bit Performance on Geothermal Well Cost," Trans., Geothermal Res. Council (Oct. 1981) 8, 261-66.

4. Carson, C.C. and Lin, Y.T.: "Geothermal Well Costs and Their Sensitivities to Changes in Drilling and Completion Operations, " Proc. Int1. Conf. Geothermal Drilling and Completion Tech., Report SAND81-0036C, Sandia Nat1. Laboratories, Albuquerque, NM (Jan. 21-23, 1981) 8-1 thru 8-26.

5. Varnado, S.G., Huff, C.F., and Yarrington, P.: "The Design and Use of Polycrystalline Diamond Compact Drag Bits in the Geothermal Environment," paper SPE 8378 presented at the 54th Annual Fall Tech. Conf. and Exhib. of the Soc. of Petr. Engr., Las Vegas, NV, Sept. 23-26, 1979.

6. Varnado, S.G., Huff, C.F., and Yarrington, P.: "Studies Aim at Optimizing Design for PDC Hard-Formation Bits," World Oil (March 1980) 6370 .

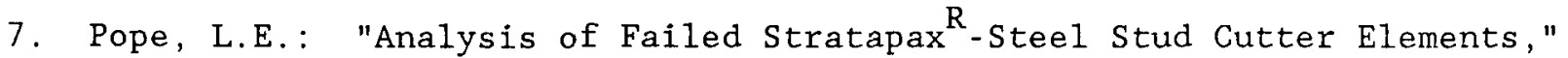
Report SAND80-0410, Sandia Natl. Laboratories, Albuquerque, NM (April 1981).

8. Huff, C.F., Ashmore, R.F., and Miller, J.W.: "Single Point Rock Cutting Strength $\mathrm{R}^{\text {and }}$ Fatigue Evaluation of Gas Pressure Diffusion Bonded Stratapax ," Report SAND77-1962, Sandia Nat1. Laboratories, Albuquerque, NM (April 1978).

9. Jellison, J.L.: "Gas Pressure Bonding of Stratapax" " ASME Paper 77 PET-72 presented at Energy Tech. Conf., Houston, TX, Sept. 18-22, 1977.

10. Huff, C.F., Jellison, J.L., and Varnado, S.G.: "Bonding Technique Attaches Stratapax to Drill Bits," The Oil and Gas Journal (Feb. 19, 1979) $111-114$.

11. Middleton, J.N. and Finger, J.T.: "Diffusion Bonding of Stratapax for Dril1 Bits," Report SAND82-2309, Sandia Nat1. Laboratories, Albuquerque, NM (January 1983).

12. Huff, C.F., McFall, A.L., and St. Clair, J.A.: "Design of Special Performance Bits Utilizing Synthetic Diamond Cutters," paper SPE 7515 presented at the 53rd Annual Fall Tech. Conf. and Exhib. of the Soc. of Petr. Engr., Houston, TX, Oct. 1-4, 1978. 
13. Huff, C.F. and Varnado, S.G: "Recent Developments in Polycrystalline Diamond Drill Bit Design," Report SAND79-1592C, Sandia Natl. Laboratories, Albuquerque, NM (May 1980); also presented at the 1980 ASME Energy-Source Tech. Conf., New Orleans, LA, Feb. 3-7, 1980.

14. Varnado, S.G., ed.: "Geothermal Drilling and Completion Technology Development Program Annual Progress Report, October 1979-September 1980," Report SAND80-2179, Sandia Nat1. Laboratories, Albuquerque, NM (Nov. 1980).

15. Hoover, E.R. and Pope, L.E.: "Failure Mechanisms of Polycrystalline Diamond Compact Drill Bits in Geothermal Environments," Report SAND81-1404, Sandia Nat1. Laboratories, Albuquerque, NM (Sept. 1981)

16. Hoover, E.R. and Middleton, J.N.: "Laboratory Evaluation of PDC Drill Bits Under High-Speed and High-Wear Conditions," J. Petr. Tech. (Dec. 1981) $2316-2321$.

17. Kelsey, J.R., ed.: "Geothermal Technology Development Program Annual Progress Report, October 1980-September 1981," Report SAND81-2124, Sandia Nat1. Laboratories, Albuquerque, NM (Sept. 1982).

18. Heckes, A.A., Meano, W., and Baker, L.E.: "Polycrystalline Diamond Drill Bits for Venezuelan Oil Field Applications," Report SAND82-1963, Sandia Natl. Laboratories, Albuquerque, NM (April 1983).

19. Hibbs, L.E., Jr. and Sogoian, G.C.: "Wear Mechanisms for Polycrystalline Diamond Compacts as Utilized for Drilling in Geothermal Environments-Final Report," Report SAND82-7213, Sandia Nat1. Laboratories, Albuquerque, NM (May 1983).

20. Glowka, D.A.: "Thermal Limitations on the Use of PDC Bits in Geothermal Drilling," Trans. Geothermal Res. Council (Aug. 1984) 8, 261-66.

21. Swenson, D.V., Wesenberg, D.L., and Jones, A.K.: "Analytical and Experimental Investigations of Rock Cutting Using Polycrystalline Diamond Compact Drag Cutters," paper SPE 10150 presented at the 56th Annual Fall Tech. Conf, and Exhib. of the Soc. of Petr. Engr., San Antonio, TX, Oct. 57,1981 .

22. Ortega, A. and Glowka, D.A.: "Studies of the Frictional Heating of Polycrystalline Diamond Compact Tools During Rock Cutting," Report SAND802677, Sandia Nat1. Laboratories, Albuquerque, NM (June 1980).

23. Glowka, D.A.: "Optimization of Bit Hydraulic Configurations," Soc. Petr. Engr. J. (Feb. 1983) 21-32.

24. Zeuch, D.H., Swenson, D.V., and Finger, J.T.: "Subsurface Damage Development in Rock During Drag-Bit Cutting: Observations and Model Predictions," Proc. 24th U.S. Symp. on Rock Mech., Texas A\&M Univ., College Station, TX, (June 20-23, 1983) 733-742. 
25. Swenson, D.V.: "Modeling and Analysis of Drag Bit Cutting," Report SAND83-0278, Sandia Nat1. Laboratories, Albuquerque, NM (July 1983).

26. Ortega, A. and Glowka, D.A.: "Frictional Heating and Convective Cooling of Polycrystalline Diamond Drag Tools During Rock Cutting," Soc. Petr. Engr. J. (April 1984) 121-128.

27. Glowka, D.A. and Stone, C.M.: "Thermal Response of Polycrystalline Diamond Compact Cutters Under Simulated Downhole Conditions," Soc. Petr. Engr. J. (April 1985) 143-156.

28. Glowka, D.A.: "Design Considerations for a Hard-Rock PDC Drill Bit," Trans. Geothermal Res. Council (Aug. 1985) 9, 123-128.

29. Glowka, D.A. and Stone, C.M.: "Effects of Thermal and Mechanical Loading on PDC Bit Life," SPE Drilling Engr. (June 1986) 201-214.

30. Zeuch, D.H. and Finger, J.T.: "Rock Breakage Mechanisms with a PDC Bit," paper SPE 14219 presented at the 60th Ann. Tech. Conf. and Exhib. of the Soc. of Petr. Engr., Las Vegas, NV, Sept. 22-25, 1985.

31. Glowka, D.A.: "Implications of Thermal Wear Phenomena for PDC Bit Design and Operation," paper SPE 14222 presented at the 60th Ann. Tech. Conf. and Exhib. of the Soc. of Petr. Engr., Las Vegas, NV, Sept. 22-25, 1985 .

32. Glowka, D.A.: "The Use of Single-Cutter Data in the Analysis of PDC Bit Designs," paper SPE 15619 presented at the 61st Annual Tech. Conf. and Exhib. of the Soc. of Petr. Engr., New Orleans, LA, Oct. 5-8, 1986.

33. Lee, M. and Hibbs, L.E., Jr.: "Role of Deformation Twin Bands in the Wear Processes of Polycrystalline Diamond Tools," Wear of Materials, K.C. Ludema, W.A. Glaeser, and S.K. Rhee, eds., ASME (1979) 485-91.

34. Hood, M.: "A Study of Methods to Improve the Performance of Drag Bits Used to Cut Hard Rock," Report 35/77, Chamber of Mines of South Africa (Aug. 1977).

35. Hood, M.: "Cutting Strong Rock with a Drag Bit Assisted by HighPressure Water Jets," J. South African Inst. Min. Met. (Nov. 1976) 79-90.

36. Dubugnon, 0.: "An Experimental Study of Water Assisted Drag Bit Cutting of Rocks," presented at 1st U.S. Water Jet Symp., Golden, CO, April $7-9,1981$.

37. Melaugh, J.F. and Saltzer, J.A.: "Development of a Predictive Model for Drilling Pressurized Shale with Stratapax Blank Bits," presented at ASME Energy Technology Conf., Houston, TX, Jan. 19-22, 1981.

38. Kelsey, J.R., ed: "Geothermal Technology Development Program Annual Progress Report, October 1983-September 1984," Report SAND85-1138, Sandia Nat1. Laboratories, Albuquerque, NM (Aug. 1985). 
39. Cortes, J. and Besson, A.: "Behavior of Polycrystalline Diamond Compact Cutters While Drilling in Bottomhole Conditions-Field Applications," Proc. Int. Conf. on Geothermal Drilling and Completion Tech., Report SAND810036C, Sandia Nat1. Laboratories, Albuquerque, NM (Jan. 21-23, 1981) 11-1 thru 11-18.

40. Walker, B., Drilling Research Laboratory, Salt Lake City, UT, private communication, April 1986.

41. Maurer, W.C.: "Bit-Tooth Penetration Under Simulated Borehole Conditions,". J. Petr. Tech. (Dec. 1965) 1433-1442.

42. Conn, A.F., Johnson, V.E., Jr., Liu, H.L., and Frederick, G.S.: "Evaluation of Cavijet Cavitating Jets for Deep-Hole Rock Cutting," Report SAND81-7067, Sandia Nat1. Laboratories, Albuquerque, NM (May 1981).

43. Johnson, V.E., Jr., Lindenmuth, W.T., Chahine, G.L., Conn, A.F., and Frederick, G.S.: "Research and Development of Improved Cavitating Jets for Deep-Hole Drilling," Report SAND83-7461, Sandia Nat1. Laboratories, Albuquerque, NM (Jan. 1984).

44. Chahine, G.L., Genoux, P.F., Johnson, V.E., Jr., and Frederick, G.S.: "Analytical and Experimental Study of the Acoustics and the Flow Field Characteristics of Cavitating Self-Resonating Water Jets, "Report SAND847142, Sandia Natl. Laboratories, Albuquerque, NM (Sept. 1984).

45. Chahine, G.L., Genoux, P.F., Liu, H.L., and Johnson, V.E., Jr.: "Analytical and Experimental Study of Self-Resonating Water Jets: NozzleJet and Wall-Jet Interactions," Report SAND86-7124, Sandia Natl. Laboratories, Albuquerque, NM (July 1987).

46. Chahine, G.L., Johnson, V.E., Jr., Kalumuck, K.M., Perdue, T.O., Waxman, D.N., Frederick, G.S., and Watson, R.E.: "Internal and Externa1 Acoustics and Large Structures Dynamics of Cavitating Self-Resonating Water Jets," Report SAND86-7176, Sandia Nat1. Laboratories, Albuquerque, NM (July 1987).

47. Jones, I.R. and Edwards, D.H.: "An Experimental Study of the Forces Generated by the Collapse of Transient Cavities in Water," J. Fluid Mech. (1960) 7, 596-609.

48. Aronson, E.A., McCaughey, K.G., and Walton, E.L.: "STRATAPAX Computer Program Update," Report SAND82-1087, Sandia Nat1. Lab., Albuquerque, NM (Sept. 1982).

49. Gill, C.W. and Martin, J.L.: "Matrix Body PDC Bits Prove Most Cost Effective in the Powder River Basin," paper IADC/SPE 13462 presented at IADC/SPE 1985 Drilling Conference, New Orleans, LA (March 1985) 341-354.

50. Gartling, D.K.: "COYOTE - A Finite Element Computer Program for Nonlinear Heat Conduction Problems," Report SAND77-1332 (Revised), Sandia Natl. Laboratories, Albuquerque, NM (Oct. 1982). 
51. Robertson, E.: "Thermal Conductivities of Rocks," USGS Open-File Report 79-356 (1979).

52. Mondy, L. and Duda, L.: "Advanced Wellbore Thermal Simulator GEOTEMP2 User Manual," Report SAND84-0857, Sandia Natl. Laboratories, Albuquerque, NM (Nov. 1984). 
APPENDIX A

EXPERIMENTAL SINGLE-CUTTER DATA 
TABLE A-1

DESCRIPTION OF CUTTERS USED IN LABORATORY TESTS

\begin{tabular}{lccccc} 
CUTTER & $\begin{array}{c}\mathrm{A}_{\mathrm{w}} \\
\left(\mathrm{in}^{2}\right)\end{array}$ & $\mathrm{w}$ & $\mathrm{L}$ & $\begin{array}{c}\text { COMPACT } \\
\text { DIAMETER } \\
\text { (in) }\end{array}$ & $\begin{array}{c}\text { WEARFLAT } \\
\text { TYPE * }\end{array}$ \\
\hline & & (in) & (in) & & \\
$\mathrm{A}$ & 0.016 & 0.220 & 0.090 & 0.50 & $\mathrm{~F}$ \\
$\mathrm{~B}$ & 0.017 & 0.240 & 0.070 & 0.50 & $\mathrm{~F}$ \\
$\mathrm{C}$ & 0.017 & 0.340 & 0.050 & 0.50 & $\mathrm{~F}$ \\
$\mathrm{D}$ & 0.020 & 0.320 & 0.100 & 0.75 & $\mathrm{~L}$ \\
$\mathrm{E}$ & 0.022 & 0.220 & 0.140 & 0.50 & $\mathrm{~F}, \mathrm{~L}$ \\
$\mathrm{~F}$ & $0.030-0.040$ & $0.300-0.320$ & $0.140-0.180$ & 0.50 & $\mathrm{M}, \mathrm{L}$ \\
$\mathrm{G}$ & 0.032 & 0.360 & 0.130 & 0.75 & $\mathrm{~L}$ \\
$\mathrm{H} * *$ & 0.029 & 0.340 & 0.120 & 0.75 & $\mathrm{~L}$ \\
$\mathrm{I}$ & 0.040 & 0.320 & 0.180 & 0.50 & $\mathrm{M}$ \\
$\mathrm{J}$ & 0.000 & 0.000 & 0.000 & 0.50 & $\mathrm{~S}$ \\
$\mathrm{~K}$ & 0.000 & 0.00 & 0.00 & 0.75 & $\mathrm{~S}$
\end{tabular}

* $\mathrm{F}=$ field-worn; $\mathrm{L}=$ laboratory-worn; $\mathrm{M}=$ machine ground; $\mathrm{S}=$ sharp.

** Cutter $\mathrm{H}$ used in waterjet-assisted cuts. Al1 cutters had a backrake angle of $20^{\circ}$. 
TABLE $A-2$

DRY, NON-INTERACTING CUT TEST DATA FOR BEREA SANDSTONE

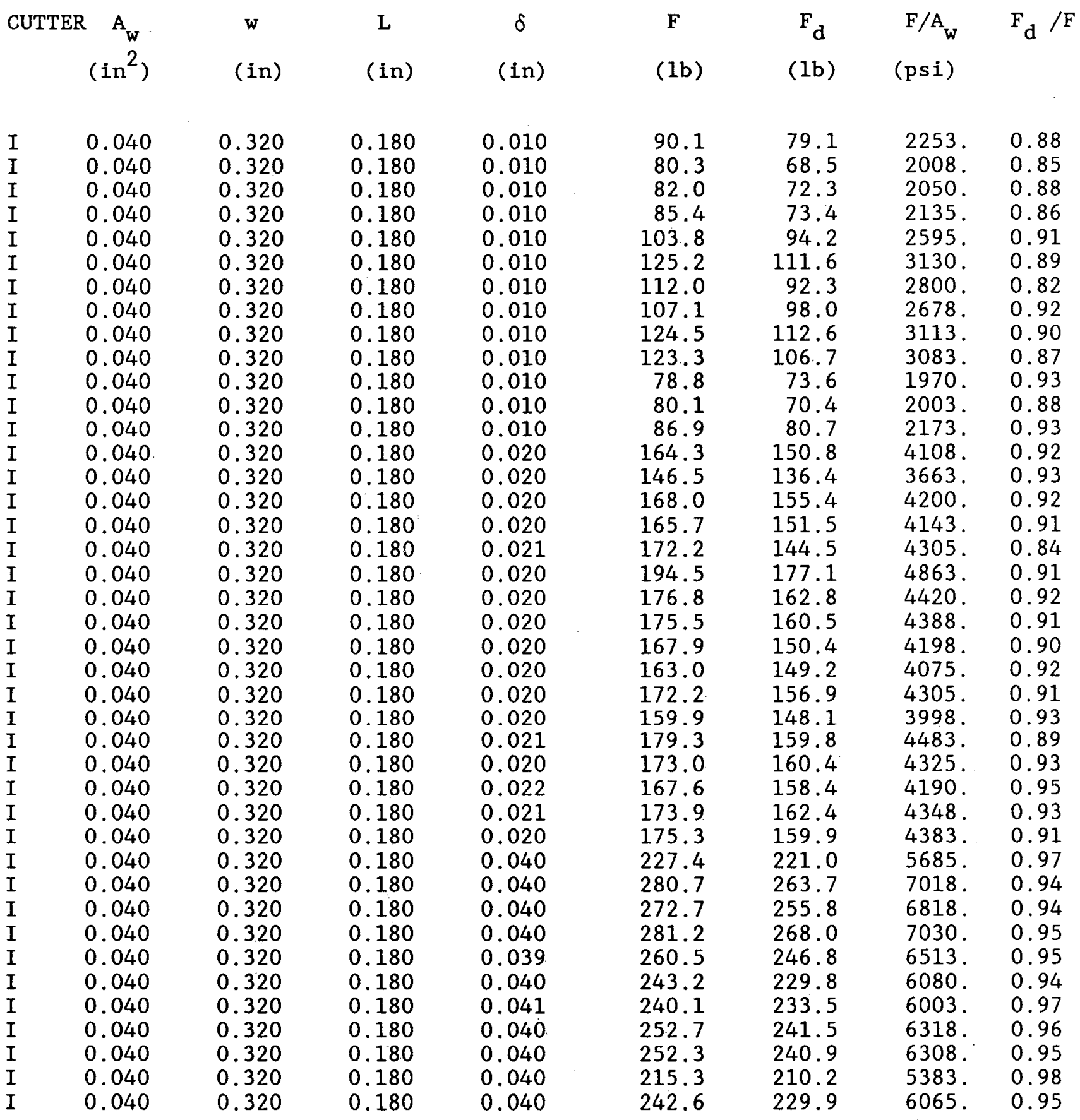


TABLE A-2 (CONT'D)

\begin{tabular}{|c|c|c|c|c|c|c|c|}
\hline $\begin{array}{r}\text { CUTTER } A_{w} \\
\left(i n^{2}\right)\end{array}$ & (in) & (in) & (in) & (1b) & $\begin{array}{c}F_{d} \\
(1 b)\end{array}$ & $\begin{array}{l}F / A_{w} \\
(p s i)\end{array}$ & $\mathrm{F}_{\mathrm{d}} / \mathrm{F}$ \\
\hline 0.040 & 0.320 & 0.180 & 0.040 & 249.0 & 235.3 & 6225 . & 0.94 \\
\hline 0.040 & 0.320 & 0.180 & 0.040 & 255.9 & 247.8 & & 0.97 \\
\hline 0.040 & 0.320 & 0.180 & 0.040 & 272.9 & 263.7 & 6823. & 0.97 \\
\hline 0.040 & 0.320 & 0.180 & 0.040 & 279.0 & 268.2 & 6975. & 0.96 \\
\hline 0.040 & 0.320 & 0.180 & 0.040 & 237.3 & 233.6 & 5933. & 0.98 \\
\hline 0.040 & 0.320 & 0.180 & 0.040 & 258.6 & 247.1 & 6465. & 0.96 \\
\hline 0.040 & 0.320 & 0.180 & 0.040 & 248.5 & 233.8 & 6213. & 0.94 \\
\hline 0.040 & 0.320 & 0.180 & 0.040 & 230.0 & 222.3 & 5750 . & 0.97 \\
\hline 0.040 & 0.320 & 0.180 & 0.041 & 240.5 & 236.8 & 6013. & 0.98 \\
\hline 0.040 & 0.320 & 0.180 & 0.041 & 264.4 & 249.5 & 6610. & 0.94 \\
\hline 0.040 & 0.320 & 0.180 & 0.041 & 226.5 & 219.5 & 5663. & 0.97 \\
\hline 0.040 & 0.320 & $0: 180$ & 0.041 & 230.0 & 223.4 & 5750 . & 0.97 \\
\hline 0.040 & 0.320 & 0.180 & 0.042 & 248.5 & 239.0 & 6213. & 0.96 \\
\hline 0.040 & 0.320 & 0.180 & 0.040 & 235.6 & 229.6 & 5890. & 0.97 \\
\hline 0.040 & 0.320 & 0.180 & 0.040 & 228.4 & 223.1 & 5710. & 0.98 \\
\hline 0.040 & 0.320 & 0.180 & 0.041 & 241.2 & 229.4 & 6030. & 0.95 \\
\hline 0.040 & 0.320 & 0.180 & 0.041 & 264.7 & 250.0 & 6618 . & 0.94 \\
\hline 0.040 & 0.320 & 0.180 & 0.041 & 229.9 & 227.4 & 5748 . & 0.99 \\
\hline 0.040 & 0.320 & 0.180 & 0.060 & 290.0 & 285.1 & 7250. & 0.98 \\
\hline 0.040 & 0.320 & 0.180 & 0.060 & 285.5 & 289.3 & 7138 . & 1.01 \\
\hline 0.040 & 0.320 & 0.180 & 0.060 & 314.3 & 313.3 & 7858. & 1.00 \\
\hline 0.040 & 0.320 & 0.180 & 0.060 & 299.8 & 301.8 & 7495. & 1.01 \\
\hline 0.040 & 0.320 & 0.180 & 0.062 & 289.9 & 292.0 & 7248 . & 1.01 \\
\hline 0.040 & 0.320 & 0.180 & 0.061 & 294.4 & 293.0 & 7360 & 1.00 \\
\hline 0.040 & 0.320 & 0.180 & 0.061 & 290.1 & 286.5 & 7253. & 0.99 \\
\hline 0.040 & 0.320 & 0.180 & 0.061 & 312.7 & 310.8 & 7818 . & 0.99 \\
\hline 0.040 & 0.320 & 0.180 & 0.060 & 286.2 & 295.2 & 7155. & 1.03 \\
\hline 0.040 & 0.320 & 0.180 & 0.080 & 355.9 & 365.8 & 8898 . & 1.03 \\
\hline 0.040 & 0.320 & 0.180 & 0.080 & 379.5 & 400.0 & 9488. & 1.05 \\
\hline 0.040 & 0.320 & 0.180 & 0.082 & 326.3 & 323.1 & 8158 . & 0.99 \\
\hline 0.040 & 0.320 & 0.180 & 0.080 & 379.5 & 400.0 & 9488. & 1.05 \\
\hline 0.040 & 0.320 & 0.180 & 0.080 & 319.9 & 326.1 & 7998. & 1.02 \\
\hline 0.040 & 0.320 & 0.180 & 0.082 & 352.7 & 363.3 & 8818: & 1.03 \\
\hline 0.040 & 0.320 & 0.180 & 0.082 & 350.8 & 383.2 & 8770 . & 1.09 \\
\hline 0.040 & 0.320 & 0.180 & 0.080 & 366.4 & 375.5 & 9160. & 1.02 \\
\hline 0.040 & 0.320 & 0.180 & 0.080 & 365.2 & 365.3 & 9130. & 1.00 \\
\hline 0.040 & 0.320 & 0.180 & 0.080 & 373.4 & 371.4 & 9335. & 0.99 \\
\hline 0.040 & 0.320 & 0.180 & 0.080 & 362.7 & 356.8 & 9068. & 0.98 \\
\hline 0.040 & 0.320 & 0.180 & 0.080 & 376.3 & 376.5 & 9408 . & 1.00 \\
\hline 0.040 & 0.320 & 0.180 & 0.100 & 380.5 & 410.6 & 9513. & 1.08 \\
\hline 0.040 & 0.320 & 0.180 & 0.099 & 410.4 & 430.2 & 10260 & 1.05 \\
\hline 0.040 & 0.320 & 0.180 & 0.100 & 528.1 & 534.6 & 13203. & 1.01 \\
\hline 0.040 & 0.320 & 0.180 & 0.100 & 450.1 & 469.1 & 11253 & 1.04 \\
\hline 0.040 & 0.320 & 0.180 & 0.100 & 451.4 & 469.6 & 11285 . & 1.04 \\
\hline
\end{tabular}


TABLE A-2 (CONT'D)

\begin{tabular}{|c|c|c|c|c|c|c|c|c|}
\hline CUTTER & $\begin{array}{c}A_{w} \\
\left(i n^{2}\right)\end{array}$ & $\begin{array}{l}\text { w } \\
\text { (in) }\end{array}$ & (in) & (in) & (1b) & $\begin{array}{c}F_{d} \\
(1 b)\end{array}$ & $\begin{array}{l}F / A_{w} \\
\text { (psi) }\end{array}$ & $\mathrm{F}_{\mathrm{d}} / \mathrm{F}$ \\
\hline I & 0.040 & 0.320 & 0.180 & 0.100 & 427.0 & 450.0 & 10675. & 1.05 \\
\hline $\bar{I}$ & 0.040 & 0.320 & 0.180 & 0.100 & 464.9 & 476.6 & 11623 & 1.03 \\
\hline I & 0.040 & 0.320 & 0.180 & 0.100 & 395.6 & 417.9 & 9890 & 1.06 \\
\hline $\bar{I}$ & 0.040 & 0.320 & 0.180 & 0.100 & 479.0 & 505.8 & 11975. & 1.06 \\
\hline $\mathrm{J}$ & 0.000 & 0.000 & 0.000 & 0.023 & 13.3 & 19.4 & $\ldots$ & 1.46 \\
\hline $\mathrm{J}$ & 0.000 & 0.000 & 0.000 & 0.020 & 16.9 & 21.7 & $\ldots$ & 1.28 \\
\hline $\mathrm{J}$ & 0.000 & 0.000 & 0.000 & 0.022 & 17.3 & 21.0 & -- & 1.21 \\
\hline$J$ & 0.000 & 0.000 & 0.000 & 0.019 & 18.6 & 23.6 & $\ldots$ & 1.27 \\
\hline $\mathrm{J}$ & 0.000 & 0.000 & 0.000 & 0.042 & 60.1 & 66.0 & $\ldots$ & 1.10 \\
\hline $\mathrm{J}$ & 0.000 & 0.000 & 0.000 & 0.041 & 62.8 & 75.6 & $\ldots$ & 1.20 \\
\hline $\mathrm{J}$ & 0.000 & 0.000 & 0.000 & 0.041 & 58.1 & 71.7 & $\ldots$ & 1.23 \\
\hline $\mathrm{J}$ & 0.000 & 0.000 & 0.000 & 0.041 & 57.9 & 67.5 & - & 1.17 \\
\hline $\mathrm{J}$ & 0.000 & 0.000 & 0.000 & 0.080 & 117.0 & 143.0 & $\cdots$ & 1.22 \\
\hline $\mathrm{J}$ & 0.000 & 0.000 & 0.000 & 0.082 & 116.0 & 139.0 & $\ldots$ & 1.20 \\
\hline $\mathrm{J}$ & 0.000 & 0.000 & 0.000 & 0.078 & 128.0 & 154.0 & -- & 1.20 \\
\hline $\mathrm{J}$ & 0.000 & 0.000 & 0.000 & 0.060 & 94.5 & 110.0 & $\ldots$ & 1.16 \\
\hline $\mathrm{J}$ & 0.000 & 0.000 & 0.000 & 0.062 & 91.3 & 106.0 & $\cdots$ & 1.16 \\
\hline B & 0.017 & 0.240 & 0.070 & 0.011 & 45.2 & 41.7 & 2659 . & 0.92 \\
\hline B & 0.017 & 0.240 & 0.070 & 0.011 & 46.9 & 41.2 & 2759 . & 0.88 \\
\hline B & 0.017 & 0.240 & 0.070 & 0.011 & 44.4 & 39.3 & 2612 . & 0.89 \\
\hline B & 0.017 & 0.240 & 0.070 & 0.012 & 39.8 & 39.0 & 2341. & 0.98 \\
\hline B & 0.017 & 0.240 & 0.070 & 0.012 & 42.8 & 40.0 & 2518 . & 0.93 \\
\hline B & 0.017 & 0.240 & 0.070 & 0.013 & 43.2 & 37.7 & 2541 . & 0.87 \\
\hline B & 0.017 & 0.240 & 0.070 & 0.018 & 58.1 & 52.4 & 3418 . & 0.90 \\
\hline B & 0.017 & 0.240 & 0.070 & 0.020 & 54.5 & 49.8 & 3206 . & 0.91 \\
\hline B & 0.017 & 0.240 & 0.070 & 0.020 & 56.5 & 51.5 & 3324 . & 0.91 \\
\hline B & 0.017 & 0.240 & 0.070 & 0.020 & 58.3 & 56.2 & 3429 . & 0.96 \\
\hline B & 0.017 & 0.240 & 0.070 & 0.025 & 60.5 & 63.8 & 3559 . & 1.05 \\
\hline B & 0.017 & 0.240 & 0.070 & 0.043 & 102.0 & 106.0 & 6000. & 1.04 \\
\hline B & 0.017 & 0.240 & 0.070 & 0.044 & 102.0 & 110.0 & 6000. & 1.08 \\
\hline B & 0.017 & 0.240 & 0.070 & 0.045 & 106.0 & 112.0 & 6235. & 1.06 \\
\hline B & 0.017 & 0.240 & 0.070 & 0.043 & 110.0 & 116.0 & 6471. & 1.05 \\
\hline B & 0.017 & 0.240 & 0.070 & 0.045 & 102.0 & 107.0 & 6000 . & 1.05 \\
\hline B & 0.017 & 0.240 & 0.070 & 0.045 & 105.0 & 108.0 & 6176. & 1.03 \\
\hline B & 0.017 & 0.240 & 0.070 & 0.043 & 109.0 & 113.0 & 6412 . & 1.04 \\
\hline B & 0.017 & 0.240 & 0.070 & 0.081 & 176.0 & 195.0 & 10353. & 1.11 \\
\hline B & 0.017 & 0.240 & 0.070 & 0.082 & 166.0 & 193.0 & 9765 & 1.16 \\
\hline B & 0.017 & 0.240 & 0.070 & 0.082 & 154.0 & 169.0 & 9059. & 1.10 \\
\hline B & 0.017 & 0.240 & 0.070 & 0.082 & 138.0 & 158.0 & 8118 . & 1.14 \\
\hline B & 0.017 & 0.240 & 0.070 & 0.080 & 167.0 & 190.0 & 9824. & 1.14 \\
\hline B & 0.017 & 0.240 & 0.070 & 0.083 & 169.0 & 198.0 & 9941. & 1.17 \\
\hline $\mathrm{C}$ & 0.017 & 0.340 & 0.050 & 0.010 & 46.1 & 42.7 & 2712 . & 0.93 \\
\hline C & 0.017 & 0.340 & 0.050 & 0.013 & 47.4 & 44.5 & 2788 . & 0.94 \\
\hline
\end{tabular}


TABLE A-2 (CONT'D)

\begin{tabular}{|c|c|c|c|c|c|c|c|c|}
\hline & $\begin{array}{l}\mathrm{R}_{\mathrm{w}} \\
\left(\mathrm{in}^{2}\right)\end{array}$ & $\begin{array}{l}\text { w } \\
\text { (in) }\end{array}$ & $\begin{array}{l}\mathrm{L} \\
\text { (in) }\end{array}$ & $\begin{array}{c}\delta \\
\text { (in) }\end{array}$ & $\begin{array}{c}F \\
(1 b)\end{array}$ & $\begin{array}{c}F_{d} \\
(1 b)\end{array}$ & $\begin{array}{l}F / A_{w} \\
\text { (psi) }\end{array}$ & $\mathrm{F}_{\mathrm{d}} / \mathrm{F}$ \\
\hline C & 0.017 & 0.340 & 0.050 & 0.012 & 45.9 & 44.3 & 2700 . & 0.97 \\
\hline C & 0.017 & 0.340 & 0.050 & 0.012 & 44.3 & 46.0 & 2606 . & 1.04 \\
\hline c & 0.017 & 0.340 & 0.050 & 0.013 & 46.5 & 44.5 & 2735 . & 0.96 \\
\hline c & 0.017 & 0.340 & 0.050 & 0.012 & 41.7 & 41.5 & 2453 . & 1.00 \\
\hline C & 0.017 & 0.340 & 0.050 & 0.012 & 44.0 & 42.9 & 2588 . & 0.98 \\
\hline C & 0.017 & 0.340 & 0.050 & 0.030 & 95.8 & 101.0 & 5635. & 1.05 \\
\hline C & 0.017 & 0.340 & 0.050 & 0.030 & 97.9 & 102.0 & 5759. & 1.04 \\
\hline C & 0.017 & 0.340 & 0.050 & 0.030 & 90.1 & 96.0 & 5300. & 1.07 \\
\hline C & 0.017 & 0.340 & 0.050 & 0.029 & 93.6 & 95.0 & 5506. & 1.01 \\
\hline C & 0.017 & 0.340 & 0.050 & 0.029 & 91.2 & 96.8 & 5365. & 1.06 \\
\hline C & 0.017 & 0.340 & 0.050 & 0.029 & 95.9 & 93.2 & 5641. & 0.97 \\
\hline $\mathrm{C}$ & 0.017 & 0.340 & 0.050 & 0.029 & 97.3 & 100.0 & 5724. & 1.03 \\
\hline C & 0.017 & 0.340 & 0.050 & 0.081 & 175.0 & 202.0 & 10294 . & 1.15 \\
\hline C & 0.017 & 0.340 & 0.050 & 0.081 & 173.0 & 205.0 & 10176 & 1.18 \\
\hline C & 0.017 & 0.340 & 0.050 & 0.081 & 159.0 & 187.0 & 9353. & 1.18 \\
\hline C & 0.017 & 0.340 & 0.050 & 0.081 & 153.0 & 189.0 & 9000 . & 1.24 \\
\hline C & 0.017 & 0.340 & 0.050 & 0.081 & 172.0 & 204.0 & 10118 & 1.19 \\
\hline C & 0.017 & 0.340 & 0.050 & 0.081 & 164.0 & 192.0 & 9647. & 1.17 \\
\hline C & 0.017 & 0.340 & 0.050 & 0.081 & 182.0 & 210.0 & 10706 . & 1.15 \\
\hline A & 0.016 & 0.220 & 0.090 & 0.023 & 73.6 & 72.0 & 4600 & 0.98 \\
\hline A & 0.016 & 0.220 & 0.090 & 0.023 & 74.0 & 72.4 & 4625. & 0.98 \\
\hline A & 0.016 & 0.220 & 0.090 & 0.024 & 73.8 & 72.1 & 4613. & 0.98 \\
\hline A & 0.016 & 0.220 & 0.090 & 0.023 & 70.3 & 68.3 & 4394. & 0.97 \\
\hline A & 0.016 & 0.220 & 0.090 & 0.023 & 74.4 & 72.4 & 4650. & 0.97 \\
\hline A & 0.016 & 0.220 & 0.090 & 0.024 & 83.9 & 78.6 & 5244 . & 0.94 \\
\hline A & 0.016 & 0.220 & 0.090 & 0.023 & 85.0 & 79.2 & 5312 . & 0.93 \\
\hline A & 0.016 & 0.220 & 0.090 & 0.067 & 133.0 & 151.0 & 8313. & 1.14 \\
\hline A & 0.016 & 0.220 & 0.090 & 0.083 & 183.0 & 205.0 & 11437. & 1.12 \\
\hline A & 0.016 & 0.220 & 0.090 & 0.083 & 152.0 & 177.0 & 9500 & 1.16 \\
\hline A & 0.016 & 0.220 & 0.090 & 0.083 & 157.0 & 178.0 & 9813 & 1.13 \\
\hline A & 0.016 & 0.220 & 0.090 & 0.083 & 165.0 & 189.0 & 10313. & 1.15 \\
\hline A & 0.016 & 0.220 & 0.090 & 0.083 & 184.0 & 201.0 & 11500 . & 1.09 \\
\hline A & 0.016 & 0.220 & 0.090 & 0.082 & 170.0 & 189.0 & 10625 . & 1.11 \\
\hline$E$ & 0.022 & 0.220 & 0.140 & 0.026 & 97.9 & 94.1 & 4450 . & 0.96 \\
\hline $\mathbf{E}$ & 0.022 & 0.220 & 0.140 & 0.024 & 82.9 & 80.7 & 3768 . & 0.97 \\
\hline $\mathrm{E}$ & 0.022 & 0.220 & 0.140 & 0.024 & 87.5 & 83.8 & 3977 . & 0.96 \\
\hline $\mathrm{E}$ & 0.022 & 0.220 & 0.140 & 0.024 & 89.2 & 84.2 & 4055. & 0.94 \\
\hline$E$ & 0.022 & 0.220 & 0.140 & 0.024 & 92.1 & 85.5 & 4186. & 0.93 \\
\hline $\mathrm{E}$ & 0.022 & 0.220 & 0.140 & 0.025 & 98.1 & 94.2 & 4459. & 0.96 \\
\hline $\mathrm{E}$ & 0.022 & 0.220 & 0.140 & 0.024 & 95.7 & 89.9 & 4350 & 0.94 \\
\hline E & 0.022 & 0.220 & 0.140 & 0.085 & 235.0 & 259.0 & 10682. & 1.10 \\
\hline E & 0.022 & 0.220 & 0.140 & 0.086 & 224.0 & 241.0 & 10182. & 1.08 \\
\hline $\mathrm{E}$ & 0.022 & 0.220 & 0.140 & 0.085 & 233.0 & 263.0 & 10591. & 1.13 \\
\hline
\end{tabular}


TABLE A-2 (CONT'D)

\begin{tabular}{|c|c|c|c|c|c|c|c|c|}
\hline & $\begin{array}{l}A_{w} \\
\left(i n^{2}\right)\end{array}$ & $\begin{array}{l}\mathrm{w} \\
\text { (in) }\end{array}$ & (in) & (in) & (1b) & $\begin{array}{c}F_{d} \\
(1 b)\end{array}$ & $\begin{array}{l}F / A_{w} \\
(p s i)\end{array}$ & $F_{d} / F$ \\
\hline $\mathrm{E}$ & 0.022 & 0.220 & 0.140 & 0.084 & 200.0 & 220.0 & 9091. & 1.10 \\
\hline $\mathrm{E}$ & 0.022 & 0.220 & 0.140 & 0.084 & 219.0 & 235.0 & 9955. & 1.07 \\
\hline $\mathrm{E}$ & 0.022 & 0.220 & 0.140 & 0.085 & 214.0 & 237.0 & 9727. & 1.11 \\
\hline $\mathrm{E}$ & 0.022 & 0.220 & 0.140 & 0.085 & 219.0 & 238.0 & 9955. & 1.09 \\
\hline $\mathrm{K}$ & 0.000 & 0.000 & 0.000 & 0.021 & 18.0 & 24.3 & $\ldots$ & 1.35 \\
\hline $\mathrm{K}$ & 0.000 & 0.000 & 0.000 & 0.023 & 17.0 & 26.1 & $\cdots$ & 1.54 \\
\hline $\mathrm{K}$ & 0.000 & 0.000 & 0.000 & 0.023 & 16.7 & 19.2 & $\ldots$ & 1.15 \\
\hline K & 0.000 & 0.000 & 0.000 & 0.020 & 16.9 & 23.5 & $\cdots$ & 1.39 \\
\hline $\mathrm{K}$ & 0.000 & 0.000 & 0.000 & 0.022 & 19.6 & 23.1 & $\ldots$ & 1.18 \\
\hline $\mathrm{K}$ & 0.000 & 0.000 & 0.000 & 0.023 & 17.7 & 23.7 & -- & 1.34 \\
\hline $\mathrm{K}$ & 0.000 & 0.000 & 0.000 & 0.021 & 19.8 & 24.0 & $\ldots$ & 1.21 \\
\hline $\mathrm{K}$ & 0.000 & 0.000 & 0.000 & 0.041 & 55.5 & 72.5 & $\cdots$ & 1.31 \\
\hline $\mathrm{K}$ & 0.000 & 0.000 & 0.000 & 0.042 & 51.8 & 70.5 & - & 1.36 \\
\hline K & 0.000 & 0.000 & 0.000 & 0.042 & 53.5 & 69.0 & $\cdots$ & 1.29 \\
\hline $\mathrm{K}$ & 0.000 & 0.000 & 0.000 & 0.042 & 48.2 & 64.5 & - & 1.34 \\
\hline $\mathrm{K}$ & 0.000 & 0.000 & 0.000 & 0.041 & 53.5 & 68.0 & - . & 1.27 \\
\hline K & 0.000 & 0.000 & 0.000 & 0.041 & 49.2 & 68.3 & $\ldots$ & 1.39 \\
\hline $\mathrm{K}$ & 0.000 & 0.000 & 0.000 & 0.041 & 52.1 & 68.2 & $\cdots$ & 1.31 \\
\hline $\mathrm{K}$ & 0.000 & 0.000 & 0.000 & 0.080 & 109.0 & 147.0 & $\ldots$ & 1.35 \\
\hline $\mathrm{K}$ & 0.000 & 0.000 & 0.000 & 0.080 & 102.0 & 142.0 & - & 1.39 \\
\hline $\mathrm{K}$ & 0.000 & 0.000 & 0.000 & 0.080 & 109.0 & 143.0 & - . - & 1.31 \\
\hline $\mathrm{K}$ & 0.000 & 0.000 & 0.000 & 0.080 & 97.5 & 138.0 & - . & 1.42 \\
\hline K & 0.000 & 0.000 & 0.000 & 0.080 & 103.0 & 141.0 & -- & 1.37 \\
\hline G & 0.032 & 0.360 & 0.130 & 0.022 & 94.9 & 96.5 & 2966 . & 1.02 \\
\hline G & 0.032 & 0.360 & 0.130 & 0.021 & 98.0 & 97.0 & 3062 . & 0.99 \\
\hline G & 0.032 & 0.360 & 0.130 & 0.023 & 94.4 & 87.9 & 2950 . & 0.93 \\
\hline G & 0.032 & 0.360 & 0.130 & 0.022 & 83.6 & 82.2 & 2612 . & 0.98 \\
\hline G & 0.032 & 0.360 & 0.130 & 0.023 & 92.4 & 94.6 & 2888 . & 1.02 \\
\hline G & 0.032 & 0.360 & 0.130 & 0.024 & 95.4 & 93.6 & 2981 . & 0.98 \\
\hline G & 0.032 & 0.360 & 0.130 & 0.022 & 91.2 & 91.9 & 2850 . & 1.01 \\
\hline G & 0.032 & 0.360 & 0.130 & 0.041 & 191.0 & 196.0 & 5969. & 1.03 \\
\hline G & 0.032 & 0.360 & 0.130 & 0.042 & 188.0 & 187.0 & 5875 . & 0.99 \\
\hline G & 0.032 & 0.360 & 0.130 & 0.042 & 181.0 & 185.0 & 5656. & 1.02 \\
\hline G & 0.032 & 0.360 & 0.130 & 0.041 & 178.0 & 184.0 & 5562 . & 1.03 \\
\hline G & 0.032 & 0.360 & 0.130 & 0.041 & 182.0 & 187.0 & 5687. & 1.03 \\
\hline G & 0.032 & 0.360 & 0.130 & 0.042 & 186.0 & 190.0 & 5812 . & 1.02 \\
\hline G & 0.032 & 0.360 & 0.130 & 0.081 & 294.0 & 319.0 & 9188 . & 1.09 \\
\hline G & 0.032 & 0.360 & 0.130 & 0.082 & 280.0 & 301.0 & 8750 . & 1.08 \\
\hline G & 0.032 & 0.360 & 0.130 & 0.081 & 283.0 & 299.0 & 8844 . & 1.06 \\
\hline G & 0.032 & 0.360 & 0.130 & 0.081 & 287.0 & 313.0 & 8969. & 1.09 \\
\hline G & 0.032 & 0.360 & 0.130 & 0.081 & 297.0 & 319.0 & 9281. & 1.07 \\
\hline
\end{tabular}


TABLE A-3

DRY, NON-INTERACTING CUT TEST DATA FOR SIERRA WHITE GRANITE

\begin{tabular}{|c|c|c|c|c|c|c|c|c|}
\hline & $\begin{array}{c}A_{w} \\
\left(i^{2}\right)\end{array}$ & $\begin{array}{l}\mathrm{w} \\
\text { (in) }\end{array}$ & $\begin{array}{l}\mathrm{L} \\
\text { (in) }\end{array}$ & $\begin{array}{c}\delta \\
\text { (in) }\end{array}$ & $\begin{array}{c}F \\
(1 b)\end{array}$ & $\begin{array}{c}\mathrm{F}_{\mathrm{d}} \\
(1 \mathrm{~b})\end{array}$ & $\begin{array}{l}F / A_{w} \\
\text { (psi) }\end{array}$ & $\mathrm{F}_{\mathrm{d}} / \mathrm{F}$ \\
\hline $\mathbf{F}$ & 0.034 & 0.310 & 0.160 & 0.011 & 657.4 & 423.0 & 19335. & 0.64 \\
\hline $\mathrm{F}$ & 0.035 & 0.310 & 0.165 & 0.010 & 553.9 & 361.0 & 15826 & 0.65 \\
\hline $\mathbf{F}$ & 0.035 & 0.310 & 0.165 & 0.010 & 627.1 & 405.7 & 17917 & 0.65 \\
\hline$F$ & 0.035 & 0.310 & 0.165 & 0.010 & 602.8 & 389.5 & 17223 . & 0.65 \\
\hline F & 0.035 & 0.310 & 0.165 & 0.010 & 591.2 & 381.7 & 16891 & 0.65 \\
\hline$F$ & 0.035 & 0.310 & 0.165 & 0.010 & 717.7 & 454.0 & 20506 . & 0.63 \\
\hline F & 0.035 & 0.310 & 0.165 & 0.010 & 632.4 & 412.1 & 18069 & 0.65 \\
\hline $\mathrm{F}$ & 0.035 & 0.310 & 0.165 & 0.010 & 789.7 & 506.3 & 22563 & 0.64 \\
\hline F & 0.035 & 0.310 & 0.165 & 0.010 & 786.9 & 496.8 & 22483 . & 0.63 \\
\hline$F$ & 0.035 & 0.310 & 0.165 & 0.010 & 778.9 & 509.6 & 22254 . & 0.65 \\
\hline F & 0.035 & 0.310 & 0.165 & 0.010 & 782.8 & 500.0 & 22366 . & 0.64 \\
\hline$F$ & 0.035 & 0.310 & 0.165 & 0.010 & 795.3 & 507.0 & 22723 . & 0.64 \\
\hline F & 0.035 & 0.310 & 0.165 & 0.010 & 786.4 & 502.2 & 22469 . & 0.64 \\
\hline$F$ & 0.033 & 0.305 & 0.155 & 0.020 & 737.3 & 458.0 & 22342 . & 0.62 \\
\hline$F$ & 0.033 & 0.305 & 0.155 & 0.020 & 696.4 & 437.2 & 21103. & 0.63 \\
\hline $\mathbf{F}$ & 0.033 & 0.305 & 0.155 & 0.020 & 806.5 & 500.0 & 24439 . & 0.62 \\
\hline $\mathrm{F}$ & 0.033 & 0.305 & 0.155 & 0.020 & 740.3 & 464.7 & 22433 . & 0.63 \\
\hline $\mathbf{F}$ & 0.033 & 0.305 & 0.155 & 0.020 & 714.5 & 446.4 & 21652 . & 0.62 \\
\hline $\mathbf{F}$ & 0.033 & 0.305 & 0.155 & 0.020 & 765.5 & 485.2 & 23197. & 0.63 \\
\hline $\mathbf{F}$ & 0.033 & 0.305 & 0.155 & 0.020 & 771.3 & 475.3 & 23373 . & 0.62 \\
\hline$F$ & 0.034 & 0.310 & 0.160 & 0.020 & 879.4 & 561.6 & 25865 . & 0.64 \\
\hline 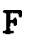 & 0.034 & 0.310 & 0.160 & 0.020 & 887.2 & 544.7 & 26094. & 0.61 \\
\hline$F$ & 0.034 & 0.310 & 0.160 & 0.020 & 896.6 & 559.5 & 26371. & 0.62 \\
\hline$F$ & 0.034 & 0.310 & 0.160 & 0.020 & 901.8 & 570.3 & 26524 . & 0.63 \\
\hline$F$ & 0.034 & 0.310 & 0.160 & 0.020 & 894.8 & 546.1 & 26318 . & 0.61 \\
\hline$F$ & 0.034 & 0.310 & 0.160 & 0.020 & 921.8 & 576.0 & 27112 . & 0.62 \\
\hline$F$ & 0.034 & 0.310 & 0.160 & 0.020 & 946.9 & 583.2 & 27850 & 0.62 \\
\hline $\mathbf{F}$ & 0.034 & 0.310 & 0.160 & 0.037 & 1220.4 & 777.3 & 35894 . & 0.64 \\
\hline $\mathbf{F}$ & 0.034 & 0.310 & 0.160 & 0.039 & 1216.9 & 783.9 & 35791. & 0.64 \\
\hline $\mathbf{F}$ & 0.034 & 0.310 & 0.160 & 0.040 & 1236.2 & 786.5 & 36359 . & 0.64 \\
\hline$F$ & 0.034 & 0.310 & 0.160 & 0.040 & 1242.8 & 790.5 & 36553. & 0.64 \\
\hline $\mathrm{F}$ & 0.034 & 0.310 & 0.160 & 0.040 & 1204.1 & 798.0 & 35415 . & 0.66 \\
\hline $\mathrm{F}$ & 0.034 & 0.310 & 0.160 & 0.040 & 1218.7 & 774.0 & 35844 . & 0.64 \\
\hline$F$ & 0.036 & 0.310 & 0.165 & 0.038 & 1418.4 & 890.6 & 39400 . & 0.63 \\
\hline$F$ & 0.036 & 0.310 & 0.165 & 0.040 & 1456.7 & 916.7 & 40464 . & 0.63 \\
\hline$F$ & 0.036 & 0.310 & 0.165 & 0.039 & 1266.9 & 822.1 & 35192 . & 0.65 \\
\hline$F$ & 0.036 & 0.310 & 0.165 & 0.038 & 1372.3 & 890.4 & 38119 . & 0.65 \\
\hline $\mathrm{F}$ & 0.036 & 0.310 & 0.165 & 0.040 & 1403.6 & 905.5 & 38989 . & 0.65 \\
\hline$F$ & 0.036 & 0.310 & 0.165 & 0.040 & 1299.8 & 822.8 & 36106 . & 0.63 \\
\hline $\mathrm{F}$ & 0.036 & 0.310 & 0.165 & 0.057 & 1484.6 & 1006.6 & 41239. & 0.68 \\
\hline $\mathrm{F}$ & 0.036 & 0.310 & 0.165 & 0.060 & 1356.1 & 907.8 & 37669 . & 0.67 \\
\hline
\end{tabular}


TABLE A-3 (CONT'D)

\begin{tabular}{|c|c|c|c|c|c|c|c|c|}
\hline & $\begin{array}{l}\mathrm{R}_{\mathrm{w}} \\
\left(i \mathrm{~A}^{2}\right)\end{array}$ & (in) & $\begin{array}{l}\mathrm{L} \\
\text { (in) }\end{array}$ & $\begin{array}{c}\delta \\
\text { (in) }\end{array}$ & $\begin{array}{c}F \\
(1 b)\end{array}$ & $\begin{array}{c}F_{d} \\
(1 b)\end{array}$ & $\begin{array}{l}F / A_{w} \\
\text { (psi) }\end{array}$ & $\mathrm{F}_{\mathrm{d}} / \mathrm{F}$ \\
\hline F & 0.036 & 0.310 & 0.165 & 0.058 & 1307.7 & 882.5 & 36325 . & 0.67 \\
\hline F & 0.036 & 0.310 & 0.165 & 0.060 & 1346.7 & 892.6 & 37408 . & 0.66 \\
\hline $\mathrm{F}$ & 0.036 & 0.310 & 0.165 & 0.060 & 1428.5 & 931.7 & 39681 . & 0.65 \\
\hline F & 0.036 & 0.310 & 0.165 & 0.060 & 1458.9 & 975.6 & 40525. & 0.67 \\
\hline $\mathbf{F}$ & 0.036 & 0.310 & 0.165 & 0.063 & 1518.5 & 983.3 & 42181. & 0.65 \\
\hline$F$ & 0.036 & 0.310 & 0.165 & 0.062 & 1517.9 & 1010.8 & 42164. & 0.67 \\
\hline $\mathrm{F}$ & 0.037 & 0.315 & 0.170 & 0.060 & 1486.1 & 953.3 & 40165. & 0.64 \\
\hline$F$ & 0.037 & 0.315 & 0.170 & 0.061 & 1525.7 & 1002.6 & 41235 . & 0.66 \\
\hline$F$ & 0.037 & 0.315 & 0.170 & 0.060 & 1619.2 & 1050.4 & 43762 . & 0.65 \\
\hline$F$ & 0.037 & 0.315 & 0.170 & 0.059 & 1605.0 & 1037.7 & 43378. & 0.65 \\
\hline$F$ & 0.037 & 0.315 & 0.170 & 0.078 & 1671.4 & 1140.2 & 45173. & 0.68 \\
\hline $\mathrm{F}$ & 0.037 & 0.315 & 0.170 & 0.080 & 1690.4 & 1147.1 & 45686. & 0.68 \\
\hline$F$ & 0.037 & 0.315 & 0.170 & 0.080 & 1590.8 & 1070.6 & 42995. & 0.67 \\
\hline F & 0.037 & 0.315 & 0.170 & 0.076 & 1561.5 & 1054.5 & 42203. & 0.68 \\
\hline$F$ & 0.037 & 0.315 & 0.170 & 0.080 & 1541.5 & 1041.7 & 41662 . & 0.68 \\
\hline$F$ & 0.037 & 0.315 & 0.170 & 0.078 & 1633.1 & 1083.1 & 44138. & 0.66 \\
\hline$F$ & 0.037 & 0.315 & 0.170 & 0.078 & 1567.4 & 1053.0 & 42362 . & 0.67 \\
\hline$F$ & 0.037 & 0.315 & 0.170 & 0.075 & 1585.9 & 1068.1 & 42862 . & 0.67 \\
\hline$F$ & 0.038 & 0.315 & 0.170 & 0.080 & 1569.2 & 1075.4 & 41295. & 0.69 \\
\hline F & 0.038 & 0.315 & 0.170 & 0.080 & 1565.5 & 1050.0 & 41197. & 0.67 \\
\hline F & 0.038 & 0.315 & 0.170 & 0.101 & 1774.5 & 1216.1 & 46697. & 0.69 \\
\hline$F$ & 0.038 & 0.315 & 0.170 & 0.101 & 1709.7 & 1201.6 & 44992 . & 0.70 \\
\hline $\mathrm{F}$ & 0.038 & 0.315 & 0.170 & 0.095 & 1661.3 & 1147.7 & 43718. & 0.69 \\
\hline $\mathrm{F}$ & 0.038 & 0.315 & 0.170 & 0.100 & 1708.5 & 1166.9 & 44961. & 0.68 \\
\hline $\mathrm{F}$ & 0.038 & 0.315 & 0.170 & 0.100 & 1722.1 & 1200.9 & 45318. & 0.70 \\
\hline $\mathrm{F}$ & 0.038 & 0.315 & 0.170 & 0.100 & 1759.2 & 1231.7 & 46295. & 0.70 \\
\hline$F$ & 0.038 & 0.315 & 0.170 & 0.100 & 1731.9 & 1206.4 & 45576. & 0.70 \\
\hline $\mathbf{F}$ & 0.039 & 0.315 & 0.175 & 0.095 & 1612.6 & 1138.5 & 41349. & 0.71 \\
\hline$F$ & 0.039 & 0.315 & 0.175 & 0.100 & 1589.1 & 1124.3 & 40746 . & 0.71 \\
\hline$F$ & 0.039 & 0.315 & 0.175 & 0.100 & 1653.3 & 1172.2 & 42392 . & 0.71 \\
\hline $\mathrm{J}$ & 0.000 & 0.000 & 0.000 & 0.017 & 31.7 & 26.3 & - - & 0.83 \\
\hline $\mathbf{J}$ & 0.000 & 0.000 & 0.000 & 0.015 & 25.3 & 16.8 & -- & 0.66 \\
\hline $\mathrm{J}$ & 0.000 & 0.000 & 0.000 & 0.016 & 30.4 & 17.0 & - . & 0.56 \\
\hline $\mathrm{J}$ & 0.000 & 0.000 & 0.000 & 0.018 & 29.2 & 18.8 & $\cdots$ & 0.64 \\
\hline$J$ & 0.000 & 0.000 & 0.000 & 0.013 & 23.9 & 16.9 & $\ldots$ & 0.71 \\
\hline $\mathrm{J}$ & 0.000 & 0.000 & 0.000 & 0.020 & 38.0 & 28.9 & -- & 0.76 \\
\hline$J$ & 0.000 & 0.000 & 0.000 & 0.039 & 131.0 & 118.0 & $\cdots$ & 0.90 \\
\hline$J$ & 0.000 & 0.000 & 0.000 & 0.039 & 136.0 & 120.0 & $\cdots$ & 0.88 \\
\hline $\mathrm{J}$ & 0.000 & 0.000 & 0.000 & 0.039 & 137.0 & 121.0 & - - & 0.88 \\
\hline$J$ & 0.000 & 0.000 & 0.000 & 0.037 & 136.0 & 118.0 & $-\cdots$ & 0.87 \\
\hline $\mathrm{J}$ & 0.000 & 0.000 & 0.000 & 0.061 & 229.0 & 217.0 & - - & 0.95 \\
\hline $\mathrm{J}$ & 0.000 & 0.000 & 0.000 & 0.062 & 256.0 & 227.0 & $\cdots$ & 0.89 \\
\hline
\end{tabular}


TABLE A-3 (CONT'D)

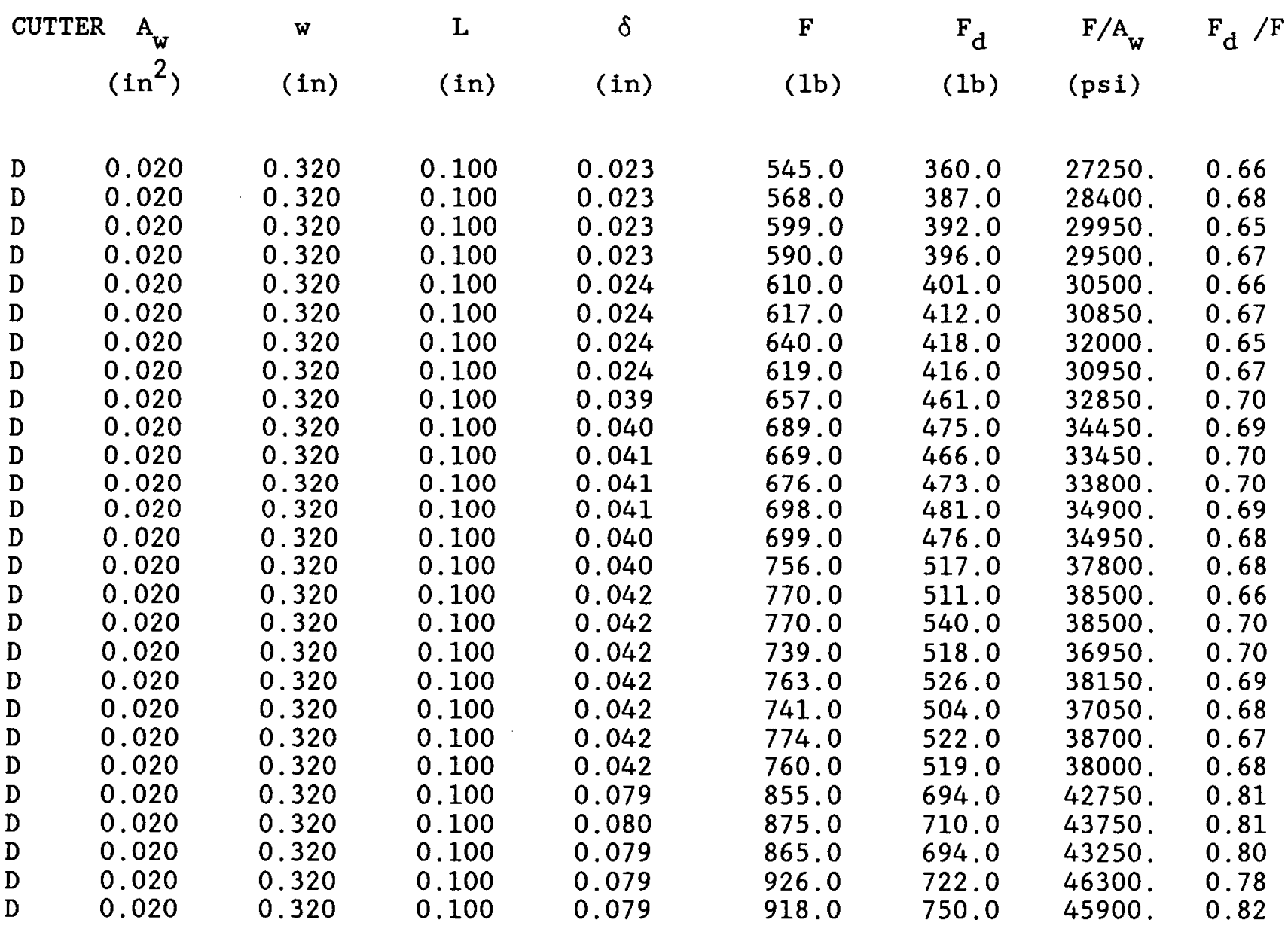


TABLE A-4

DRY, NON-INTERACTING CUT TFST DATA FOR TENNESSEE MARBLE

\begin{tabular}{|c|c|c|c|c|c|c|c|c|}
\hline & $\begin{array}{l}A_{w} \\
\left(i n^{2}\right)\end{array}$ & $\begin{array}{l}\text { w } \\
\text { (in) }\end{array}$ & (in) & (in) & (1b) & $\begin{array}{c}F_{d} \\
(1 b)\end{array}$ & $\begin{array}{l}F / A_{w} \\
(p s i)\end{array}$ & $\mathrm{F}_{\mathrm{d}} / \mathrm{F}$ \\
\hline F & 0.030 & 0.300 & 0.140 & 0.010 & 448.2 & 324.3 & 14940. & 0.72 \\
\hline$F$ & 0.030 & 0.300 & 0.140 & 0.010 & 455.2 & 322.6 & 15173 & 0.71 \\
\hline $\mathrm{F}$ & 0.030 & 0.300 & 0.140 & 0.010 & 442.1 & 318.0 & 14737 . & 0.72 \\
\hline $\mathrm{F}$ & 0.030 & 0.300 & 0.140 & 0.010 & 457.1 & 332.7 & 15237 . & 0.73 \\
\hline $\mathrm{F}$ & 0.030 & 0.300 & 0.140 & 0.010 & 474.4 & 350.0 & 15813. & 0.74 \\
\hline $\mathrm{F}$ & 0.030 & 0.300 & 0.140 & 0.010 & 527.9 & 360.6 & 17597 . & 0.68 \\
\hline F & 0.030 & 0.300 & 0.140 & 0.010 & 583.0 & 360.3 & 19433. & 0.62 \\
\hline$F$ & 0.030 & 0.300 & 0.140 & 0.010 & 461.2 & 336.8 & 15373 & 0.73 \\
\hline F & 0.030 & 0.300 & 0.140 & 0.010 & 447.7 & 325.0 & 14923. & 0.73 \\
\hline F & 0.030 & 0.300 & 0.140 & 0.010 & 460.1 & 328.6 & 15337 & 0.71 \\
\hline $\mathrm{F}$ & 0.030 & 0.300 & 0.140 & 0.010 & 501.1 & 354.7 & 16703 & 0.71 \\
\hline$F$ & 0.030 & 0.300 & 0.140 & 0.010 & 509.9 & 351.2 & 16997. & 0.69 \\
\hline$F$ & 0.030 & 0.300 & 0.140 & 0.020 & 809.6 & 464.8 & 26987 . & 0.57 \\
\hline F & 0.030 & 0.300 & 0.140 & 0.020 & 751.8 & 459.8 & 25060 . & 0.61 \\
\hline$F$ & 0.030 & 0.300 & 0.140 & 0.020 & 768.4 & 457.0 & 25613 . & 0.59 \\
\hline F & 0.030 & 0.300 & 0.140 & 0.020 & 724.8 & 472.1 & 24160 . & 0.65 \\
\hline$F$ & 0.030 & 0.300 & 0.140 & 0.020 & 740.5 & 474.1 & 24683 . & 0.64 \\
\hline$F$ & 0.030 & 0.300 & 0.140 & 0.020 & 758.2 & 467.9 & 25273 . & 0.62 \\
\hline$F$ & 0.030 & 0.300 & 0.140 & 0.020 & 734.6 & 461.1 & 24487 & 0.63 \\
\hline$F$ & 0.030 & 0.300 & 0.140 & 0.020 & 706.6 & 449.1 & 23553 . & 0.64 \\
\hline$F$ & 0.030 & 0.300 & 0.140 & 0.020 & 723.2 & 459.0 & 24107 . & 0.63 \\
\hline$F$ & 0.030 & 0.300 & 0.140 & 0.020 & 727.0 & 451.4 & 24233 . & 0.62 \\
\hline$F$ & 0.030 & 0.300 & 0.140 & 0.020 & 707.6 & 431.2 & 23587 . & 0.61 \\
\hline $\mathrm{F}$ & 0.030 & 0.300 & 0.140 & 0.019 & 761.4 & 463.8 & 25380 . & 0.61 \\
\hline F & 0.030 & 0.300 & 0.140 & 0.020 & 798.3 & 446.8 & 26610 & 0.56 \\
\hline$F$ & 0.030 & 0.300 & 0.140 & 0.018 & 777.1 & 452.4 & 25903. & 0.58 \\
\hline$F$ & 0.030 & 0.300 & 0.140 & 0.020 & 673.5 & 427.3 & 22450 . & 0.63 \\
\hline F & 0.030 & 0.300 & 0.140 & 0.038 & 842.0 & 596.0 & 28067 . & 0.71 \\
\hline $\mathrm{F}$ & 0.030 & 0.300 & 0.140 & 0.037 & 836.8 & 574.7 & 27893 . & 0.69 \\
\hline$F$ & 0.030 & 0.300 & 0.140 & 0.039 & 854.9 & 587.4 & 28497 . & 0.69 \\
\hline $\mathrm{F}$ & 0.030 & 0.300 & 0.140 & 0.039 & 854.3 & 584.3 & 28477 . & 0.68 \\
\hline $\mathrm{F}$ & 0.030 & 0.300 & 0.140 & 0.038 & 916.0 & 574.8 & 30533. & 0.63 \\
\hline$F$ & 0.030 & 0.300 & 0.140 & 0.038 & 865.2 & 523.5 & 28840 . & 0.61 \\
\hline F & 0.030 & 0.300 & 0.140 & 0.040 & 992.2 & 589.1 & 33073. & 0.59 \\
\hline$F$ & 0.030 & 0.300 & 0.140 & 0.039 & 978.0 & 573.9 & 32600 . & 0.59 \\
\hline F & 0.030 & 0.300 & 0.140 & 0.039 & 1060.7 & 605.9 & 35357 . & 0.57 \\
\hline$F$ & 0.030 & 0.300 & 0.140 & 0.039 & 1060.8 & 596.3 & 35360 . & 0.56 \\
\hline $\mathrm{F}$ & 0.030 & 0.300 & 0.140 & 0.040 & 956.2 & 528.2 & 31873 . & 0.55 \\
\hline $\mathrm{F}$ & 0.030 & 0.300 & 0.140 & 0.040 & 1143.4 & 714.2 & 38113 . & 0.62 \\
\hline $\mathrm{F}$ & 0.030 & 0.300 & 0.140 & 0.037 & 948.8 & 615.3 & 31627 & 0.65 \\
\hline$F$ & 0.030 & 0.300 & 0.140 & 0.040 & 1025.3 & 575.9 & 34177 . & 0.56 \\
\hline
\end{tabular}


TABLE A-4 (CONT'D)

\begin{tabular}{|c|c|c|c|c|c|c|c|c|}
\hline & $\begin{array}{l}R \quad A_{w} \\
\left(i n^{2}\right)\end{array}$ & $\begin{array}{l}\text { w } \\
\text { (in) }\end{array}$ & $\begin{array}{l}\mathrm{L} \\
\text { (in) }\end{array}$ & $\begin{array}{c}\delta \\
\text { (in) }\end{array}$ & $\begin{array}{c}F \\
(1 b)\end{array}$ & $\begin{array}{c}F_{\mathrm{d}} \\
(1 b)\end{array}$ & $\begin{array}{l}F / A_{w} \\
\text { (psi) }\end{array}$ & $\mathrm{F}_{\mathrm{d}} / \mathrm{F}$ \\
\hline F & 0.030 & 0.300 & 0.140 & 0.040 & 996.8 & 572.7 & 33227 . & 0.57 \\
\hline F & 0.030 & 0.300 & 0.140 & 0.040 & 1021.5 & 557.2 & 34050 . & 0.55 \\
\hline F & 0.030 & 0.300 & 0.140 & 0.040 & 1078.9 & 584.4 & 35963. & 0.54 \\
\hline F & 0.030 & 0.300 & 0.140 & 0.040 & 1106.0 & 573.0 & 36867 . & 0.52 \\
\hline$F$ & 0.030 & 0.300 & 0.140 & 0.040 & 1078.6 & 578.7 & 35953. & 0.54 \\
\hline$F$ & 0.030 & 0.300 & 0.140 & 0.058 & 1278.6 & 770.4 & 42620 & 0.60 \\
\hline F & 0.030 & 0.300 & 0.140 & 0.060 & 1107.7 & 663.1 & 36923 . & 0.60 \\
\hline F & 0.030 & 0.300 & 0.140 & 0.059 & 1114.2 & 683.4 & 37140 . & 0.61 \\
\hline F & 0.030 & 0.300 & 0.140 & 0.059 & 1131.7 & 631.1 & 37723. & 0.56 \\
\hline $\mathrm{F}$ & 0.030 & 0.300 & 0.140 & 0.060 & 1174.9 & 689.6 & 39163. & 0.59 \\
\hline $\mathrm{F}$ & 0.030 & 0.300 & 0.140 & 0.059 & 1240.8 & 738.5 & 41360 . & 0.60 \\
\hline$F$ & 0.030 & 0.300 & 0.140 & 0.059 & 1160.3 & 710.9 & 38677 . & 0.61 \\
\hline $\mathrm{F}$ & 0.030 & 0.300 & 0.140 & 0.060 & 1202.7 & 706.5 & 40090. & 0.59 \\
\hline$F$ & 0.030 & 0.300 & 0.140 & 0.060 & 1177.3 & 704.9 & 39243. & 0.60 \\
\hline $\mathbf{F}$ & 0.030 & 0.300 & 0.140 & 0.062 & 1136.2 & 694.0 & 37873 . & 0.61 \\
\hline$F$ & 0.030 & 0.300 & 0.140 & 0.059 & 1300.1 & 729.7 & 43337. & 0.56 \\
\hline$F$ & 0.030 & 0.300 & 0.140 & 0.060 & 1177.1 & 694.8 & 39237. & 0.59 \\
\hline F & 0.030 & 0.300 & 0.140 & 0.060 & 1173.4 & 669.6 & 39113. & 0.57 \\
\hline$F$ & 0.030 & 0.300 & 0.140 & 0.060 & 1200.9 & 659.6 & 40030. & 0.55 \\
\hline $\mathbf{F}$ & 0.030 & 0.300 & 0.140 & 0.060 & 1132.6 & 630.5 & 37753 . & 0.56 \\
\hline F & 0.030 & 0.300 & 0.140 & 0.060 & 1289.4 & 701.7 & 42980 . & 0.54 \\
\hline F & 0.030 & 0.300 & 0.140 & 0.060 & 1336.4 & 712.9 & 44547. & 0.53 \\
\hline$F$ & 0.030 & 0.300 & 0.140 & 0.078 & 1314.1 & 822.1 & 43803 & 0.63 \\
\hline $\mathrm{F}$ & 0.030 & 0.300 & 0.140 & 0.078 & 1384.3 & 860.9 & 46143 & 0.62 \\
\hline F & 0.030 & 0.300 & 0.140 & 0.077 & 1156.1 & 693.2 & 38537 . & 0.60 \\
\hline$F$ & 0.030 & 0.300 & 0.140 & 0.079 & 1388.8 & 862.6 & 46293 & 0.62 \\
\hline $\mathrm{F}$ & 0.030 & 0.300 & 0.140 & 0.078 & 1152.4 & 778.8 & 38413 . & 0.68 \\
\hline F & 0.030 & 0.300 & 0.140 & 0.078 & 1420.8 & 860.4 & 47360 . & 0.61 \\
\hline $\mathrm{F}$ & 0.030 & 0.300 & 0.140 & 0.080 & 1332.1 & 812.0 & 44403. & 0.61 \\
\hline F & 0.030 & 0.300 & 0.140 & 0.081 & 1427.6 & 820.3 & 47587. & 0.57 \\
\hline$F$ & 0.030 & 0.300 & 0.140 & 0.080 & 1138.6 & 700.0 & 37953. & 0.61 \\
\hline $\mathrm{F}$ & 0.030 & 0.300 & 0.140 & 0.097 & 1428.6 & 980.9 & 47620 & 0.69 \\
\hline F & 0.030 & 0.300 & 0.140 & 0.100 & 1186.0 & 800.0 & 39533. & 0.67 \\
\hline F & 0.030 & 0.300 & 0.140 & 0.100 & 1259.0 & 799.9 & 41967. & 0.64 \\
\hline F & 0.030 & 0.300 & 0.140 & 0.100 & 1566.3 & 984.7 & 52210 & 0.63 \\
\hline $\mathrm{F}$ & 0.030 & 0.300 & 0.140 & 0.096 & 1357.7 & 882.4 & 45257 & 0.65 \\
\hline$F$ & 0.030 & 0.300 & 0.140 & 0.100 & 1341.0 & 843.2 & 44700 . & 0.63 \\
\hline$F$ & 0.030 & 0.300 & 0.140 & 0.096 & 1721.5 & 1050.9 & 57383. & 0.61 \\
\hline F & 0.030 & 0.300 & 0.140 & 0.095 & 1527.5 & 923.8 & 50917 & 0.60 \\
\hline F & 0.030 & 0.300 & 0.140 & 0.100 & 1147.4 & 670.2 & 38247 . & 0.58 \\
\hline
\end{tabular}


TABLE A-5

WATERJET-ASSISTED, NON-INTERACTING CUT TEST DATA FOR SIERRA WHITE GRANITE

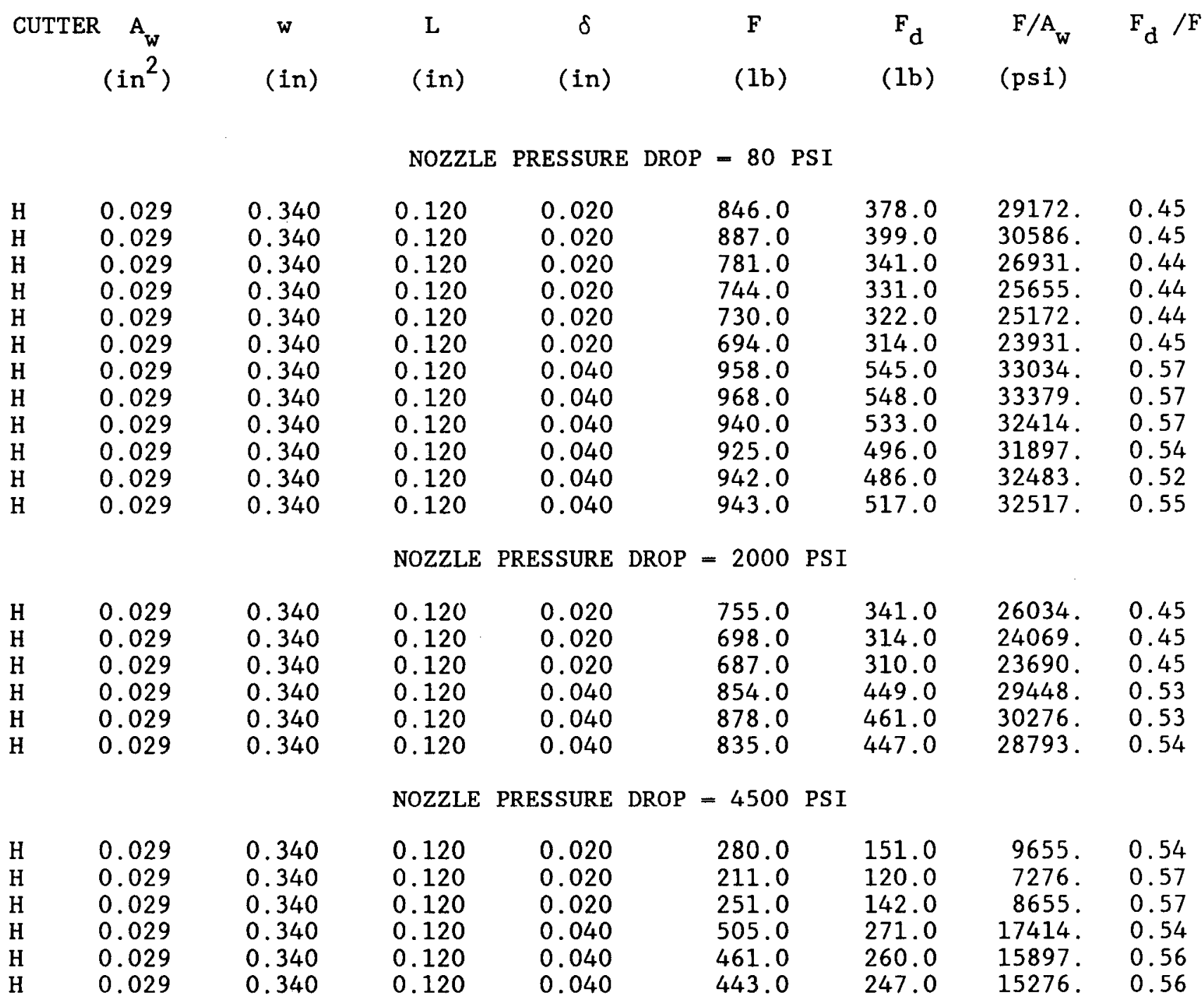


TABLE A-6

DRY, INTERACTING CUT TEST DATA FOR BEREA SANDSTONE

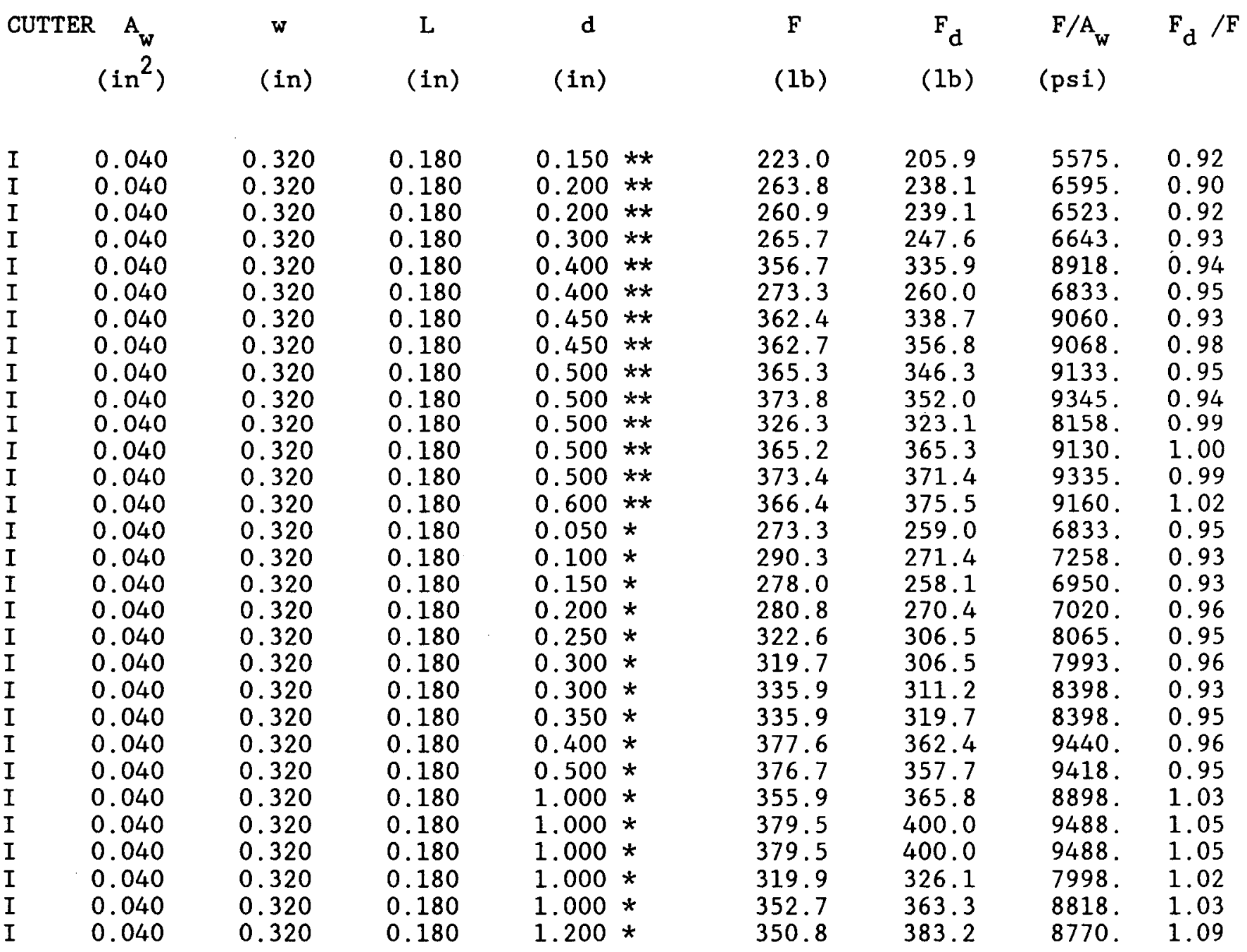

** Symmetric cutter interaction (two previous adjacent cuts)

* Asymmetric cutter interaction (one previous adjacent cut)

$\delta=0.080$ inch and $\delta_{1}=0.040$ inch in all cuts (See Figure 9.) 
TABLE A-7

DRY, INTERACTING CUT TEST DATA FOR SIERRA WHITE GRANITE

\begin{tabular}{|c|c|c|c|c|c|c|c|c|c|}
\hline & $\begin{array}{l}\mathrm{R}_{\mathrm{w}} \\
\left(i \mathrm{~A}^{2}\right)\end{array}$ & $\begin{array}{l}\text { w } \\
\text { (in) }\end{array}$ & (in) & $\begin{array}{r}d \\
\text { (in) }\end{array}$ & & (1b) & $\begin{array}{c}F_{d} \\
(1 b)\end{array}$ & $\begin{array}{c}F / A_{W} \\
\text { (psi) }\end{array}$ & $\mathrm{F}_{\mathrm{d}} / \mathrm{F}$ \\
\hline$F$ & 0.036 & 0.310 & 0.165 & 0.150 & $* *$ & 1246.7 & 801.0 & 34631. & 0.64 \\
\hline $\mathrm{F}$ & 0.036 & 0.310 & 0.165 & 0.200 & $* *$ & 1370.1 & 887.0 & 38058 . & 0.65 \\
\hline F & 0.036 & 0.310 & 0.165 & 0.250 & ** & 1381.5 & 912.0 & 38375 . & 0.66 \\
\hline F & 0.036 & 0.310 & 0.165 & 0.300 & $* *$ & 1600.6 & 1020.0 & 44461 . & 0.64 \\
\hline$F$ & 0.036 & 0.310 & 0.165 & 0.400 & $* *$ & 1557.0 & 991.0 & 43250 . & 0.64 \\
\hline F & 0.036 & 0.310 & 0.165 & 0.450 & $* *$ & 1507.6 & 1005.0 & 41878 . & 0.67 \\
\hline F & 0.040 & 0.320 & 0.180 & 0.500 & $* *$ & 1740.1 & 1101.6 & 43503. & 0.63 \\
\hline$F$ & 0.037 & 0.315 & 0.170 & 0.500 & $* *$ & 1590.8 & 1070.6 & 42995 . & 0.67 \\
\hline F & 0.037 & 0.315 & 0.170 & 0.600 & $* *$ & 1541.5 & 1041.7 & 41662 . & 0.68 \\
\hline $\mathrm{F}$ & 0.038 & 0.315 & 0.170 & 0.600 & $* *$ & 1569.2 & 1075.4 & 41295. & 0.69 \\
\hline F & 0.034 & 0.310 & 0.160 & 0.050 & $*$ & 1340.7 & 822.0 & 39432 . & 0.61 \\
\hline$F$ & 0.034 & 0.310 & 0.160 & 0.050 & * & 1210.7 & 808.0 & 35609 . & 0.67 \\
\hline$F$ & 0.034 & 0.310 & 0.160 & 0.100 & * & 1262.9 & 855.0 & 37144 . & 0.68 \\
\hline $\mathrm{F}$ & 0.034 & 0.310 & 0.160 & 0.150 & * & 1302.7 & 850.0 & 38315 . & 0.65 \\
\hline F & 0.034 & 0.310 & 0.160 & 0.200 & * & 1483.9 & 994.0 & 43644 . & 0.67 \\
\hline F & 0.034 & 0.310 & 0.160 & 0.250 & * & 1390.9 & 920.0 & 40909. & 0.66 \\
\hline $\mathrm{F}$ & 0.034 & 0.310 & 0.160 & 0.300 & * & 1387.1 & 928.0 & 40797. & 0.67 \\
\hline F & 0.034 & 0.310 & 0.160 & 0.400 & * & 1480.1 & 1010.0 & 43532 . & 0.68 \\
\hline $\mathrm{F}$ & 0.038 & 0.315 & 0.170 & 0.800 & * & 1565.5 & 1050.0 & 41197. & 0.67 \\
\hline $\mathbf{F}$ & 0.037 & 0.315 & 0.170 & 0.800 & * & 1633.1 & 1083.1 & 44138. & 0.66 \\
\hline F & 0.037 & 0.315 & 0.170 & 1.000 & * & 1690.4 & 1147.1 & 45686 . & 0.68 \\
\hline F & 0.037 & 0.315 & 0.170 & 1.000 & * & 1585.9 & 1068.1 & 42862 . & 0.67 \\
\hline F & 0.037 & 0.315 & 0.170 & 1.200 & * & 1561.5 & 1054.5 & 42203 . & 0.68 \\
\hline F & 0.037 & 0.315 & 0.170 & 1.250 & * & 1567.4 & 1053.0 & 42362 . & 0.67 \\
\hline F & 0.037 & 0.315 & 0.170 & 1.250 & * & 1671.4 & 1140.2 & 45173. & 0.68 \\
\hline
\end{tabular}

** Symmetric cutter interaction (two previous adjacent cuts)

$\delta=0.080$ inch and $\delta_{1}=0.040$ inch in all cuts (See Figure 9.) 
APPENDIX B

LISTING OF FORTRAN PROGRAM PDCWEAR 
PROGRAM PDCWEAR

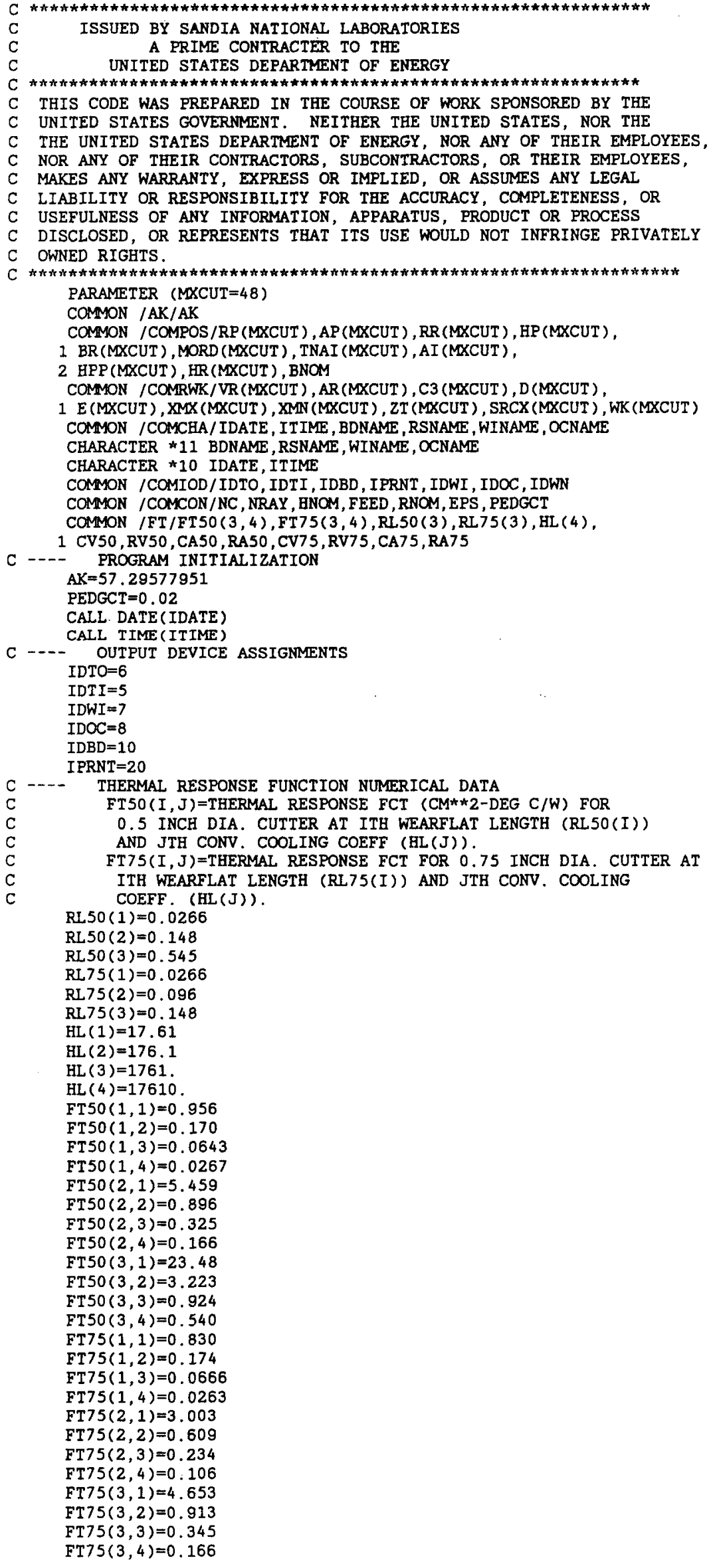


C -.- CONSTANTS AND EXPONENTS IN WEARFLAT LENGTH VS. WEAR VOLUME CV $50=2,94$

$\operatorname{RV} 50=0.40$

$\mathrm{CA} 50=1.59$

RA50 $=0.68$

CV75 $=2.72$

RV7 $5=0.40$

CA75 $=1.38$

RA75 $=0.68$

WRITE (IDTO, 100)

100 FORMAT ('

1 ,

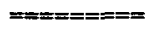

SANDIA NATIONAL LABORATORIES'/

GEOTHERMAL TECHNOLOGY DEVELOPMENT DIVISION'/ ALBUQUERQUE, NEW MEXICO $/ /$ JUNE $1987^{\prime} / /$

RSNAME $=$

BDNAME $=$ '

WINAME $=$,

OCNAME $=$

WNNAME $=$,

CALL DRIVER

END

SUBROUTINE DRIVER

C --- THIS SUBROUTINE PERFORMS THE FOLLOWING FUNCTIONS :

1) ALLOWS USER TO SPECIFY OUTPUT FILE NAMES

2) CALLS INPUT, WHICH READS INPUT DATA FROM FILEC

3) SETS UP LOOP TO RUN PROGRAM FOR 5 DIFFERENT ROP'S

4) SETS UP LOOP IN PROGRAM TO ITERATE ON CUTTER WEAR ANGLES

5) CALLS VACMP, WHICH PERFORMS GEOMETRY CALCULATIONS

6) CALLS DATPRI, WHICH PRINTS RESULTS

PARAMETER (MXCUT $=48$ )

COMMON /AK/AK

COMMON /CTWEAR/AWMAX, NCMAX, ASUBWN, MODW, WRA (MXCUT) ,

1 FAMAX, XBCAMAX, DLH, C6

COMYN /COMPOS/RP (MXCUT), AP (MXCUT), RR (MXCUT), HP (MXCUT),

1 BR(MXCUT), MORD (MXCUT), TNAI (MXCUT), AI (MXCUT)

2 HPP (MXCUT), HR (MXCUT), BNOM

PARAMETER (MINRAY $=20$ )

REAL *8 N1A, N2A, N1B, N2B

COMMON /COMCON/NC, NRAY, HNOM, FEED, RNOM, EPS, PEDGCT

COMMON /COMCHA/IDATE, ITIME, BDNAME, RSNAME, WINAME, OCNAME

CHARACTER *11 BDNAME, RSNAME, WINAME, OCNAME, WNNAME

CHARACTER $\star 10$ IDATE, ITIME

CHARACTER *20 ROCK

COMMON /COMIOD/IDTO, IDTI, IDBD, IPRNT, IDWI , IDOC, IDWN

COMMN /RESULTS/XW1 (MXCUT), XW2 (MXCUT), WL (MXCUT)

1 YOWORN (MXCUT), ITRAC , 2B (MXCUT), DE (MXCUT), NDE (MXCUT),

2 FP(MXCUT), WOB (5), BMX (5), BMY (5), FR (MXCUT), FV(MXCUT), FX (5)

3 FY(5), TORQ ( 5), TW(MXCUT), WR (MXCUT), FD (MXCUT)

4 WW(MXCUT), AW(MXCUT), IW1, XC1 (MXCUT), XC2 (MXCUT), ZC1 (MXCUT)

5 ZC2 (MXCUT), WA (MXCUT), WAO (MXCUT), ZW1 (MXCUT), ZW2 (MXCUT).

6 TWMAX (5), WRMAX (5), PWNP (MXCUT), ZBC (MXCUT) , XBC (MXCUT), IT1

7 IC1 1 IC2, IC3, IC4, IC5, IC6, BF 1, BF2, BF3, BF4, BF 5, ITYP (MXCUT) , IP1, IP2

COMON /ROCKDAT/ROCK, RK, RX, FC, DC, DCS, C1A, N1A, C1B,

2 N1B, C2A, N2A, C2B, N2B, TFL, RPM, ROP (5), NROP, IR

DIMENSION FVL (MXCUT), FRL (MXCUT)

DIMENSION IWK (MXCUT)

CHARACTER * 1 AIN

C TRACER "ON" TURNS ON MANY "WRITE" STATEMENTS--USED FOR DEBUGGING

TYPE * 'TURN TRACER ON $(Y=1, N=2)$ '

READ (5, 4)ITRAC

FORMAT (I1)

ITRAC $=2$

IF (ITRAC .EQ , 1)THEN

OPEN (UNIT $=3$, NAME= 'DIAG.DAT', TYPE $=$ 'NEW' )

ENDIF

C -.. READ INPUT DATA

CALL INPUT

27 WRITE (IDTO, 104)

104 FORMAT (/' DO YOU WISH TO TERMINATE THIS SESSION? ( $\left.Y, N)^{\prime}\right)$

CALL READA (M, AIN)

IF (M.EQ.1.OR. (AIN.NE. 'Y' , AND. AIN. NE. 'N') ) GOTO 27

IF (AIN.EQ. 'Y') CALL STOPP

C --- NAME OUTPUT DISK FILE

29 WRITE (IDTO, 106)

106 FORMAT ( $/$ ' ENTER NAME OF OUTPUT DISK FILE FOR STORING'

1 'COMPUTED RESULTS',

2 ' (11 CBAR. OR LESS, INCLUDING .EXTENSION):') 


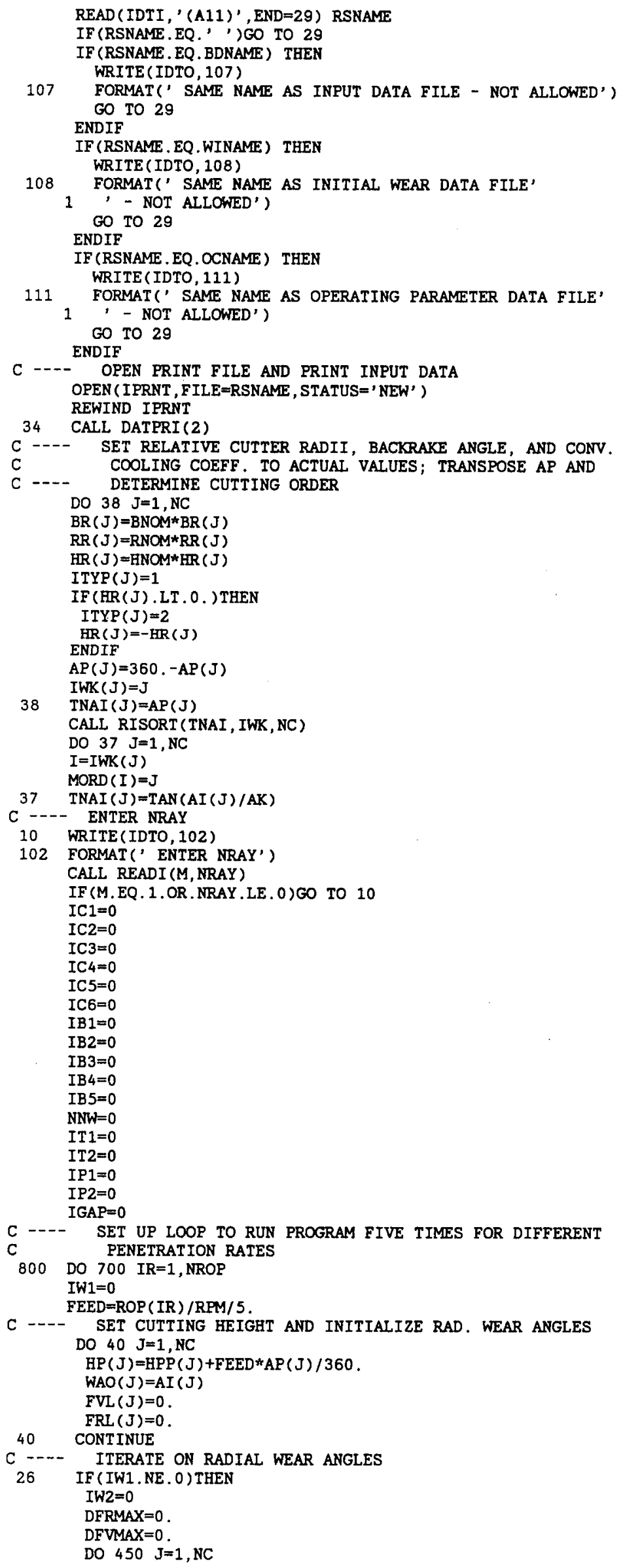




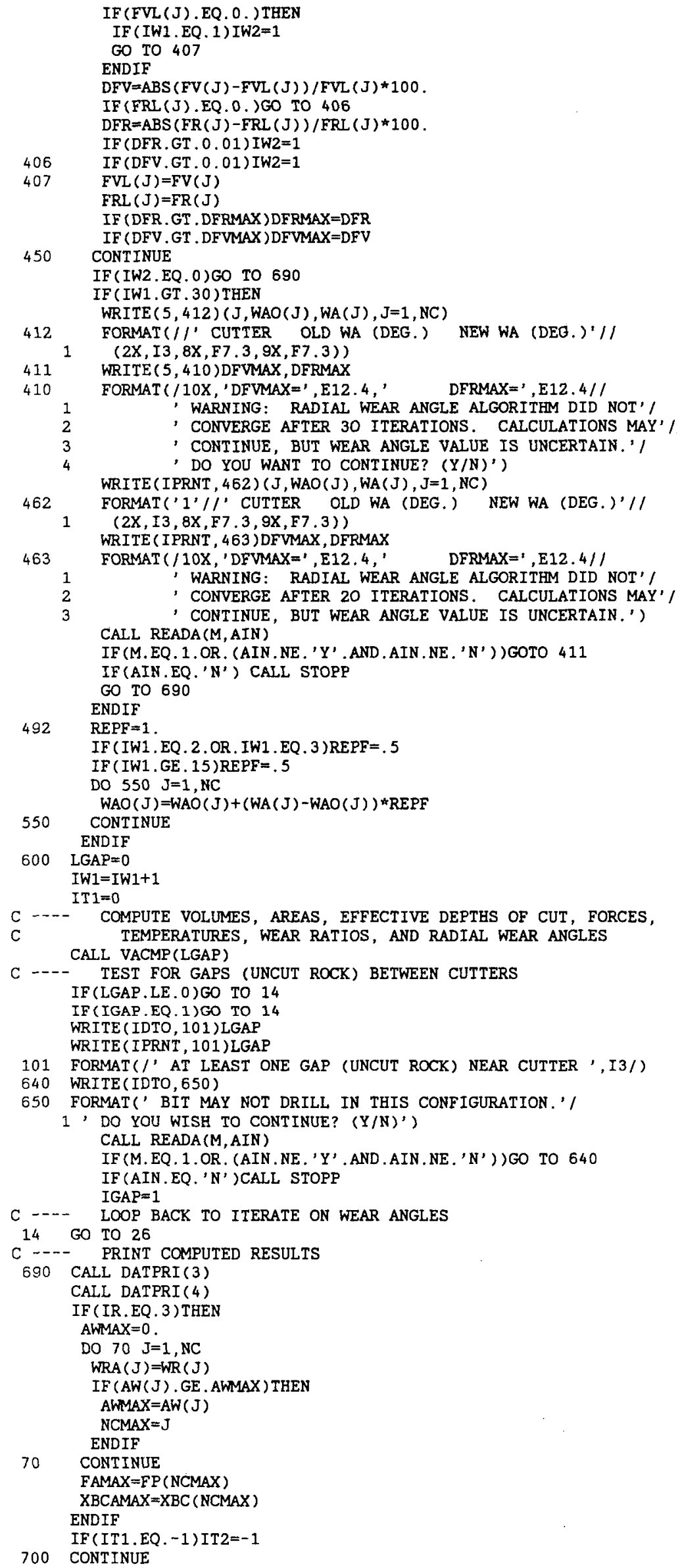


CALL DATPRI ( 7$)$

IF (IT2 . EQ . - 1) THEN

WRITE (IDTO, 820)

820 FORMAT(' AT LEAST ONE CUTTER EXPERIENCES THERMALLY-'

1 'ACCELERATED WEAR' /' AT ONE OR MORE OF THE SPECIFIED ',

2 'PENETRATION RATES.' $/$ ' ADVANCED WEAR CALCULATIONS FOR '

3 'THOSE CUTTERS WILL NOT BE ACCURATE.')

IT $2=0$

WRITE (IPRNT , 822)

822 FORMAT $(1 \mathrm{X}, / / / /)$

WRITE (IPRNT, 820)

ENDIF

805 WRITE (IDTO, 810)

810 FORMAT( /' DO YOU WISE TO REPEAT THE CALCULATIONS'

1 ' WITH MORE ADVANCED CUTTER WEAR ? (Y/N ')'

CALI READA(M, AIN)

IF (M.EQ. 1. OR. (AIN. NE. 'Y' AND. AIN. NE. 'N') )GO TO 805

IF (AIN, EQ. ' $Y$ ') THEN

CALI CUTWEAR

929 WRITE (IDTO, 906)

906 FORMAT(' ENTER NAME OF OUTPUT DISK FILE'

1 'FOR STORING NEW WEAR CONFIGURATION'

2 ' ( 11 CHAR. OR LESS, INCLUDING .EXTENSION):')

READ (IDTI,' (A11)', END=929) WNNAME

IF (WNNAME. EQ.' ') GO TO 929

IF (WNNAME . EQ. BDNAME) THEN

WRITE (IDTO, 907)

907 FORMAT(' SAME NAME AS INPUT DATA FILE - NOT ALLOWED')

GO TO 929

ENDIF

IF (WNNAME. EQ. WINAME) THEN

WRITE (IDTO, 908)

908 FORMATS' SAME NAME AS PREVIOUS WEAR DATA FILE'

1 , - NOT ALLOWED')

GO TO 929

ENDIF

IF (WNNAME . EQ . OCNAME) THEN

WRITE (IDTO, 911)

911 FORMAT(' SAME NAME AS OPERATING PARAMETER DATA FILE'

1 ' - NOT ALLOWED')

GO TO 929

ENDIF

IF (WNNAME . EQ . RSNAME) THEN

WRITE (IDTO, 912)

912 FORMAT(' SAME NAME AS OUTPUT DISK FILE FOR RESULTS'

' - NOT ALLOWED' )

GO TO 929

ENDIF

WINAME $=$ WNNAME

$\mathrm{NNW}=\mathrm{NNW}+1$

$I D W N=N N W+30$

OPEN ( IDWN , FILE=WINAME, STATUS=' NEW' $)$

CALL DATPRI ( 9$)$

CLOSE (IDWN)

210 WRITE (IDTO, 220)

220 FORMAT ( 1 ' DO YOU WISH TO PROCEED WITH THIS'

1 ' CONFIGURATION? ( $\mathrm{Y} / \mathrm{N})$ '')

CALL READA(M,AIN)

IF (M.EQ. 1.OR. (AIN .NE, 'Y' AND . AIN.NE . 'N') ) GOTO 210

IF (AIN.EQ.' 'N') CALL STOPP

GO TO 800

ENDIF

CALL STOPP

END

SUBROUTINE VACMP(LGAP)

C --- COMPUTES VOLUMES, AREAS, EFFECTIVE DEPTHS OF CUT,

C FORCES AND MOMENTS, WEARFLAT TEMPERATURES, WEAR RATIOS, AND WEAR ANGLES

PARAMETER (MXCUT $=48$ )

REAL * \& N1A, N2A, N1B , N2B

COMMON /AK/AK

COMON /CTWEAR/AWMAX, NCMAX, ASUBWN, MODW, WRA (MXCUT),

1 FAMAX, XBCAMAX, DLH, C6

CHARACTER *20 ROCK

COMMON /COMIOD/IDTO, IDTI, IDBD, IPRNT, IDWI, IDOC, IDWN

COMON /COMPOS/RP (MXCUT), AP (MXCUT), RR (MXCUT), HP (MXCUT)

1 BR (MXCUT), MORD (MXCUT), TNAI (MXCUT), AI (MXCUT)

2 HPP (MXCUT) ; HR (MXCUT), BNOM

COMMON /COMRWK/VR (MXCUT), AR (MXCUT), C3 (MXCUT), D (MXCUT)

1 E (MXCUT), XMX (MXCUT), XMN (MXCUT), ZT (MXCUT), SRCX (MXCUT), WK (MXCUT)

COMON /COMCON/NC, NRAY, HNOM, FEED, RNOM, EPS, PEDGCT

COMMON /RESULTS/XW1 (MXCUT), XW2 (MXCUT), WL (MXCUT)

1 YOWORN (MXCUT), ITRAC , ZB (MXCUT) , DE (MXCUT) , NDE (MXCUT), 
2 FP(MXCUT), WOB ( 5), BMX (5), BMY (5), FR(MXCUT), FV(MXCUT), FX (5),

3 FY(5), TORQ (5), TW(MXCUT), WR(MXCUT), FD(MXCUT),

4 WW(MXCUT), AW(MXCUT), IW1, XC1 (MXCUT), XC2 (MXCUT), ZC1 (MXCUT),

5 ZC2 (MXCUT), WA (MXCUT), WAO (MXCUT), ZW1 (MXCUT), ZW2 (MXCUT),

6 TWMAX (5), WRMAX (5), PWNP (MXCUT), ZBC (MXCUT), XBC (MXCUT), IT1,

7 IC1, IC2 , IC3 , IC4 , IC5, IC6, BF 1, BF2, BF3 , BF4, BF5, ITYP (MXCUT), IP1, IP2

COMMON /ROCKDAT/ROCK, RK, RX, FC, DC, DCS , C1A, N1A , C1B,

2 N1B, C2A, N2A, C2B, N2B, TFL, RPM, ROP ( 5), NROP, IR

COMMON /FT/FT5O (3, 4), FT75 (3, 4), RL5O(3), RL75 (3), HL (4),

1 CV50, RV50, CA50, RA50, CV75, RV75, CA75, RA75

DIMENSION KT (MXCUT), KXM(MXCUT)

C - - PEDGCT*RNOM/NRAY IS THE CLOSENESS OF RAY TO EDGE OF CUTTER DTST $=$ PEDGCT*RNOM/FLOAT $($ NRAY $)$

$\mathrm{ZMIN}=\mathrm{HP}(1)-\mathrm{RR}(1)$

$X M I N=R P(1)$

$X M A X=R P(1)$

C - - COMPUTE CUTTER COEFFICIENTS

TWMAX (IR) $=0$

WRMAX $(I R)=0$.

DO $20 \mathrm{~J}=1$, NC

$\operatorname{KXM}(\mathrm{J})=\mathrm{J}$

$\mathrm{ZMIN}=A M I N 1(Z M I N, \operatorname{HP}(J)-R R(J))$

$\operatorname{VR}(\mathrm{J})=0$

$\operatorname{AR}(J)=0$

$B=R R(J) * \cos (B R(J) / A K)$

$\operatorname{CSAI}=\operatorname{COS}(\operatorname{ATAN}(\operatorname{TNAI}(\mathrm{J})))$

SNAI $=$ SIN (ATAN (TNAI $(J)))$

$E(J)=(R R(J) * C S A I) * * 2+\left(B^{\star}\right.$ SNAI $) * \star 2$

$D(J)=-C S A I * \operatorname{SNAI} *(R R(J) * * 2-B * * 2) /(R R(J) * B)$

C3 $(J)=(R R(J) * B) / E(J)$

$B=\operatorname{SQRT}(E(J))-D T S T$

C - $\quad$ XMN $(J)$ AND XMX (J) ARE LOWER AND UPPER LIMITS OF J-TH CUTTER $X M X(J)=R P(J)+B$

$X M N(J)=A M A X 1(0, R P(J)-B)$

IF (ITRAC .EQ. 1 )WRITE (3, 309) J, XMN (J), XNX (J)

309 FORMAT(' XMN(',I3,')=',F10.5,' XMX(J)=',F10.5)

XMIN $=$ AMIN $1(X M I N, X M N(J))$

$X M A X=A M A X 1(X M A X, X M X(J))$

$\mathrm{CSB}=\mathrm{COS}(\mathrm{BR}(\mathrm{J}) / \mathrm{AK})$

$\operatorname{PWNP}(J)=A K * A T A N(\operatorname{TAN}(W A O(J) / A K-\operatorname{ATAN}(\operatorname{TNAI}(J))) / \mathrm{CSB})$

$S N P W N P=S I N(P W N P(J) / A K)$

CSPWNP $=\operatorname{COS}(\operatorname{PWNP}(\mathrm{J}) / \mathrm{AK})$

$R W=(R R(J) * * 2-(W W(J) / 2) * * 2). * \star .5$

$\mathrm{XBCNP}=\mathrm{RW}$ * SNPWNP

$Z B C N P=R W * C S P W N P$

$\mathrm{ZBCN}=\mathrm{ZBCNP} \times \mathrm{CSB}$

$\mathrm{XBC}(\mathrm{J})=\mathrm{RP}(\mathrm{J})+\mathrm{XBCNP} * \mathrm{CSAI}+\mathrm{ZBCN} * \mathrm{SNAI}$

$Z B C(J)=H P(J)-X B C N P * S N A I+Z B C N * C S A I$

$X W 1 N=X B C N P-W W(J) / 2$, *CSPWNP

$Z W 1 N=Z B C N P+W W(J) / 2$ * *SNPWNP

$\mathrm{ZW} 1 \mathrm{~N}=\mathrm{ZW} 1 \mathrm{~N} * \mathrm{CSB}$

$X W 2 N=X B C N P+W W(J) / 2 . * C S P W N P$

$Z W 2 N=Z B C N P-W W(J) / 2$. "SNPWNP

$Z W 2 \mathrm{~N}=\mathrm{ZW} 2 \mathrm{~N} * \mathrm{CSB}$

$X W 1(J)=R P(J)+X W 1 N * C S A I+Z W 1 N * S N A I$

$Z W 1(J)=H P(J)-X W 1 N * S N A I+Z W 1 N * C S A I$

$X W 2(J)=R P(J)+X W 2 N * C S A I+Z W 2 N * S N A I$

$Z W 2(J)=H P(J)-X W 2 N * S N A I+Z W 2 N * C S A I$

$\mathrm{DE}(\mathrm{J})=0$.

$\operatorname{NDE}(J)=0$

$\mathrm{XC1}(\mathrm{J})=-100$.

$\mathrm{XC} 2(\mathrm{~J})=-100$

$\mathrm{ZC} 1(\mathrm{~J})=-100$

$\mathrm{ZC} 2(\mathrm{~J})=-100$

20 CONTINUE

C --. SET BOUNDS FOR COMPUTATION

$\mathrm{ZMIN}=\mathrm{ZMIN}-10$. FEED

$\mathrm{XMN}(\mathrm{NC}+1)=\mathrm{XMAX}+\mathrm{RNOM}$

$\mathrm{KXM}(\mathrm{NC}+1)=\mathrm{NC}+1$

C -.- COMPUTE RAY STEP SIZE

DX=RNOM/FLOAT (NRAY)

$N X=I N T((X M A X-X M I N) / D X+.5)$

$\mathrm{DX}=(\mathrm{XMAX}-\mathrm{XMIN}) /$ FLOAT $(\mathrm{NX})$

$\mathrm{XO}=\mathrm{XMIN}-.5^{* \mathrm{DX}}$

CALL RISORT (XMN, KXM, NC+1)

$\mathrm{K} 1=1$

C -.- LOOP TO COMPUTE Z-VALUES FOR EACH ELEMENT DX

DO $40 \mathrm{NNX}=1, \mathrm{NX}$

$\mathrm{X}=\mathrm{XO}+\mathrm{NNX} * \mathrm{DX}$

$X V O L=6.2831853072 * X * D X$

$\mathrm{ZM}=\mathrm{ZMIN}$

$\mathrm{LS}=0$

C - - - FIND CUTTER INTERSECTIONS WITH RAY NORMAL TO X-AXIS 


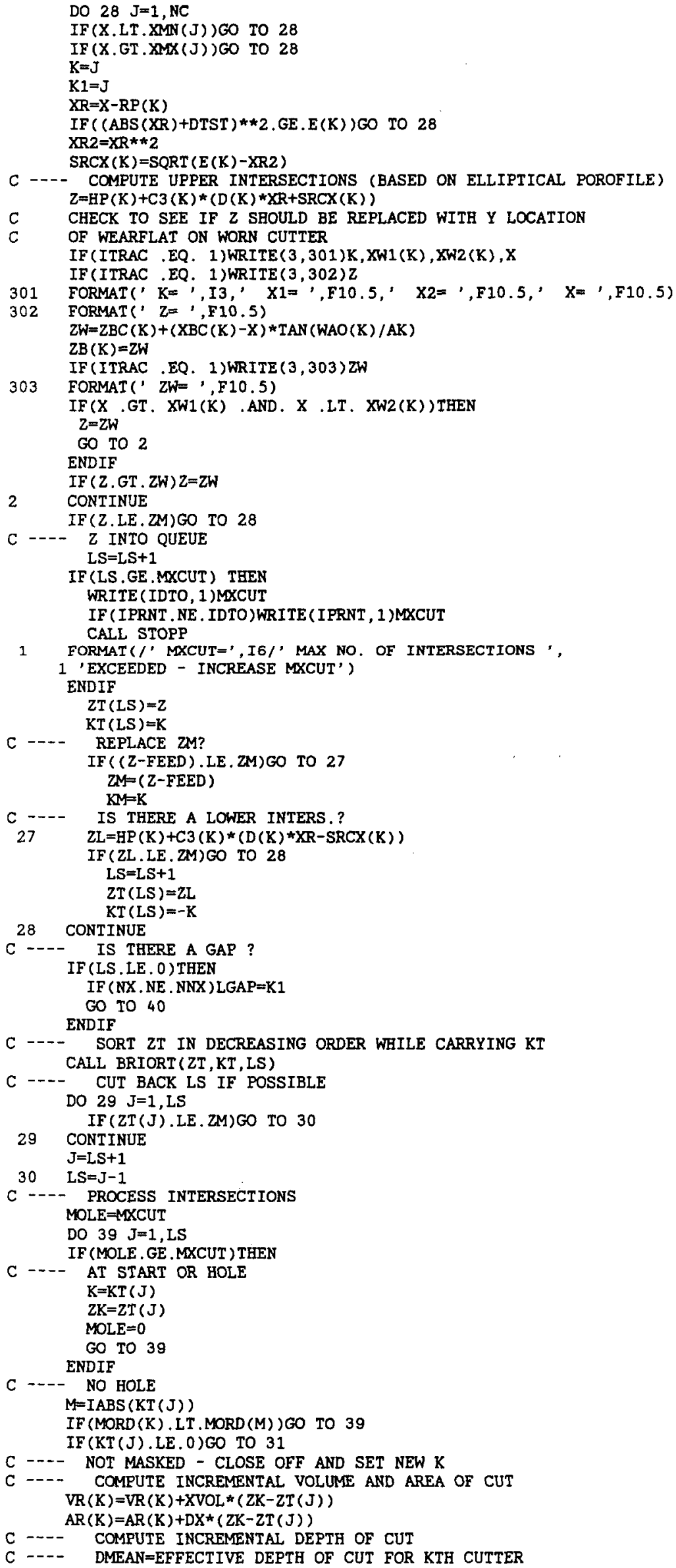


C - - NDMEAN=NUMBER OF SAMPLES IN DETERMINING DMEAN FOR EACH CUTTER $\mathrm{DE}(\mathrm{K})=\mathrm{DE}(\mathrm{K}) * \mathrm{NDE}(\mathrm{K})+(\mathrm{ZB}(\mathrm{K})-\mathrm{ZT}(\mathrm{J}))$

$1 \quad{ }^{*} \operatorname{COS}(W A O(K) / A K)$

$\mathrm{NDE}(\mathrm{K})=\mathrm{NDE}(\mathrm{K})+1$

$D E(K)=D E(K) / N D E(K)$

IF $(X C 1(K)$. EQ. -100 . ) THEN

$\mathrm{XC1}(\mathrm{K})=\mathrm{X}$

$\mathrm{ZC} 1(\mathrm{~K})=\mathrm{ZK}$

ENDIF

$\mathrm{XC} 2(\mathrm{~K})=\mathrm{X}$

$\mathrm{ZC} 2(\mathrm{~K})=\mathrm{ZK}$

IF (ITRAC .EQ, 1)WRITE (3,307)XC1(K), ZC1(K),

$1 \mathrm{XC2}(\mathrm{K}), \mathrm{ZC2}(\mathrm{K})$

307 FORMAT(' XCONT $1=,, F 10.5, ' \quad Z C O N T 1=, F 10.5$,

1 ' $X$ CONT2 $=$ ',F10.5,' ZCONT2=',F10.5)

IF (ITRAC .EQ. 1)WRITE (3,304)ZK, J, ZT (J), ZB (K)

304 FORMAT(' ZK=',F10.5,' ZT (', I3,') =',F10.5,' YBOK=',F10.5)

IF(ITRAC EQ 1) WRITE (3,305)K, VR(K), AR(K), DE (K)

305 FORMAT(' $\mathrm{VR}\left({ }^{\prime}, \mathrm{I3},,^{\prime}\right)=$, F $10.5,{ }^{\prime} \quad \mathrm{AR}(\mathrm{K})=,, \mathrm{F} 10.5$,

1. DMEAN $=$, F10.5)

$2 \mathrm{~K}=\mathrm{ZT}(\mathrm{J})$

$\mathrm{K}=\mathrm{M}$

GO TO 39

C -... UNDERCUT

C - COMPUTE INCREMENTAL VOLUME AND AREA OF CUT

$31 \quad \operatorname{VR}(\mathrm{K})=\mathrm{VR}(\mathrm{K})+\mathrm{XVOL} *(\mathrm{ZK}-\mathrm{ZT}(\mathrm{J}))$

$\operatorname{AR}(K)=A R(K)+D X^{*}(Z K-Z T(J))$

C -.. COMPUTE INCREMENTAL DEPTH OF CUT

C - DMEAN=EFFECTIVE DEPTH OF CUT FOR KTH CUTTER

C -.. NDMEAN=NUMBER OF SAMPLES IN DETERMINING DMEAN FOR EACH CUTTER $\mathrm{DE}(\mathrm{K})=\mathrm{DE}(\mathrm{K})$ *NDE $(\mathrm{K})+(\mathrm{ZB}(\mathrm{K})-\mathrm{ZT}(\mathrm{J}))$

1 $\star \operatorname{COS}(W A O(K) / A K)$

$\operatorname{NDE}(K)=\mathrm{NDE}(\mathrm{K})+1$

$\mathrm{DE}(\mathrm{K})=\mathrm{DE}(\mathrm{K}) / \mathrm{NDE}(\mathrm{K})$

IF $(X C 1(K) . E Q .-100$. ) THEN

$\mathrm{XC} 1(\mathrm{~K})=\mathrm{X}$

$\mathrm{ZC} 1(\mathrm{~K})=\mathrm{ZK}$

ENDIF

$\mathrm{XC} 2(\mathrm{~K})=\mathrm{x}$

$\mathrm{ZC} 2(\mathrm{~K})=\mathrm{ZK}$

IF (ITRAC .EQ. 1)WRITE (3, 307) XC1(K), ZC1(K),

$1 \mathrm{XC2}(\mathrm{K}), 2 \mathrm{C} 2(\mathrm{~K})$

IF(ITRAC .EQ. 1)WRITE (3, 304)ZK, J,ZT(J), ZB(K)

IF(ITRAC ,EQ. 1)WRITE (3,305)K, VR(K), AR(K),DE(K)

$\mathrm{ZK}=\mathrm{ZT}(\mathrm{J})$

C - - FIND IF HOLE

MOLE $=$ MXCUT

DO $32 \mathrm{~L}=1, \mathrm{~J}$

$I=K T(L)$

IF (I.LE. O)GO TO 32

$Y I=H P(I)+C 3(I) *(D(I) *(X-R P(I))-S R C X(I))$

IF (YI .GE .ZK. OR.MORD (I) .GT.MOLE.OR. I. EQ.K)GO TO 32

$M=I$

32 CONTINUE

IF (MOLE . EQ.MXCUT)GO TO 39

C - - NOT A HOLE

$\mathrm{K}=\mathrm{M}$

39 CONTINUE

IF (MOLE. EQ. MXCUT) GO TO 40

C -.- FINISH.

C -.. COMPUTE INCREMENTAL VOLUME AND AREA OF CUT

$\mathrm{VR}(\mathrm{K})=\mathrm{VR}(\mathrm{K})+\mathrm{XVOL} *(\mathrm{ZK}-\mathrm{ZM})$

$\mathrm{AR}(\mathrm{K})=\mathrm{AR}(\mathrm{K})+\mathrm{DX} *(\mathrm{ZK}-\mathrm{ZM})$

C - - COMPUTE INCREMENTAL DEPTH OF CUT

C DMEAN=EFFECTIVE DEPTH OF CUT FOR KTH CUTTER

NDMEAN=NUMBER OF SAMPLES IN DETERMINING DMEAN FOR EACH CUTTER $\mathrm{DE}(\mathrm{K})=\mathrm{DE}(\mathrm{K}) * \mathrm{NDE}(\mathrm{K})+(\mathrm{ZB}(\mathrm{K})-\mathrm{ZM})$

$1 \quad * \operatorname{COS}($ WAO $(K) / A K)$

$\mathrm{NDE}(\mathrm{K})=\mathrm{NDE}(\mathrm{K})+1$

$\mathrm{DE}(\mathrm{K})=\mathrm{DE}(\mathrm{K}) / \mathrm{KDE}(\mathrm{K})$

IF $(\mathrm{XC1}(\mathrm{K})$. EQ. -100 .) THEN

$\mathrm{XC1}(\mathrm{K})=\mathrm{X}$

$\mathrm{ZC1}(\mathrm{K})=\mathrm{ZK}$

ENDIF

$\mathrm{XC} 2(K)=\mathrm{X}$

$\mathrm{ZC} 2(\mathrm{~K})=\mathrm{ZK}$

IF (ITRAC .EQ. 1)WRITE (3,307)XC1(K), ZC1(K),

$1 \mathrm{XC} 2(\mathrm{~K}), \mathrm{ZC2}(\mathrm{K})$

IF (ITRAC .EQ, 1)WRITE (3,306)ZK, J, ZM, ZB (K)

306 FORMAT(' $\mathrm{ZK}=$, F10.5,' ZM(',I3,')=,,F10.5,' $\mathrm{YBOK}=$, ,F10.5)

IF (ITRAC EQ. 1)WRITE (3,305)K, VR(K), AR(K),DE(K)

40 CONTINUE 
C --- COMPUTE CUTTER FORCES, INCREMENTAL BIT FORCES, WOB (IR) $=0$.

$\operatorname{TORQ}(I R)=0$.

$\operatorname{BMX}(I R)=0$.

$B M Y(I R)=0$.

FX(IR) $=0$.

$F Y(I R)=0$.

DO $1000 \mathrm{~J}=1$, NC

C -...- $\star \star \star \star \star$ CUTTER FORCE MODELS (PENETRATING AND DRAG) $\pi * \hbar * \star$ TMPFLG $=0$

$\operatorname{IF}(\operatorname{ITYP}(J), E Q \cdot 1) \operatorname{THEN}$

$F P(J)=A W(J) * C 1 A^{*} D E(J) * * N 1 A$

$F P S=C 2 A * D E(J) * \star N 2 A$

IF (FPS.GT .FP(J)) THEN

$F P(J)=F P S$

TMPFLG $=1$

ENDIF

GO TO 1500

ENDIF

$F P(J)=A W(J) * C 1 B * D E(J) * * N 1 B$

$F P S=C 2 B * D E(J) * * N 2 B$

IF (FPS.GT.FP(J) ) THEN

$F P(J)=F P S$

TMPFLG $=1$

ENDIF

$1500 \mathrm{FD}(\mathrm{J})=\mathrm{FP}(\mathrm{J}) * \mathrm{DC}$

$\operatorname{IF}(\operatorname{AW}(J)$ EQ 0 , ) FD $(J)=F P(J) * D C S$

C - - -

C --- COMPUTE FORCE COMPONENTS, INTEGRATED FORCES, AND

C INTEGRATED MOMENTS

$\operatorname{SNAP}=\operatorname{SIN}(A P(J) / A K)$

$\operatorname{CSAP}=\operatorname{COS}(\operatorname{AP}(\mathrm{J}) / \mathrm{AK})$

$F V(J)=-F P(J) \star C O S(W A O(J) / A K)$

WOB (IR) $=$ WOB (IR $)+(-F V(J))$

$F R(J)=-F P(J) * \operatorname{SIN}(W A O(J) / A K)$

$\mathrm{T} 1=\mathrm{FR}(\mathrm{J}) * \mathrm{CSAP}$

$F X(I R)=F X(I R)+T 1$

$\mathrm{T} 1=\mathrm{FD}(\mathrm{J}) * \mathrm{SNAP}$

$\mathrm{FX}(\mathrm{IR})=\mathrm{FX}(\mathrm{IR})+\mathrm{T} 1$

$T 2=F R(J) * S N A P$

$F Y(I R)=F Y(I R)+T 2$

$\mathrm{T} 2=\mathrm{FD}(\mathrm{J}) *(-\mathrm{CSAP})$

$F Y(I R)=F Y(I R)+T 2$

$\operatorname{TORQ}(I R)=\operatorname{TORQ}(\mathrm{IR})+\mathrm{FD}(\mathrm{J}) * \mathrm{XBC}(\mathrm{J}) / 12$

$\mathrm{T} 1=\mathrm{FV}(\mathrm{J}) * \mathrm{XBC}(\mathrm{J})$ *SNAP

IF (ITRAC .EQ. 1)WRITE $(3,2000)$ T 1

2000 FORMAT(' T1=',F10.2)

$\mathrm{BMX}(\mathrm{IR})=\mathrm{BMX}(\mathrm{IR})+\mathrm{T} 1 / 12$.

$Z B C P=Z B C(J)-F E E D * A P(J) / 360$

$T 1=F D(J) * C S A P * Z B C P$

IF(ITRAC .EQ. 1)WRITE $(3,2000)$ T1

$\mathrm{BMX}(\mathrm{IR})=\mathrm{BMX}(\mathrm{IR})+\mathrm{T} 1 / 12$.

$T 1=-F R(J) * S N A P * Z B C P$

IF(ITRAC .EQ. 1)WRIIE (3, 2000)T1

$\mathrm{BMX}(\mathrm{IR})=\mathrm{BMX}(\mathrm{IR})+\mathrm{T} 1 / 12$.

$T 2=-F V(J) \star X B C(J) * C S A P$

IF(ITRAC .EQ. 1)WRITE $(3,2001) \mathrm{T2}$

2001 FORMAT (' T2=',F10.2)

$B M Y(I R)=B M Y(I R)+T 2 / 12$.

$\mathrm{T} 2=\mathrm{FD}(\mathrm{J}){ }^{*} \mathrm{SNAP}$ * $\mathrm{ZBCP}$

IF (ITRAC .EQ. 1)WRITE (3, 2001)T2

$B M Y(I R)=B M Y(I R)+T 2 / 12$.

$T 2=F R(J) * C S A P \star Z B C P$

IF (ITRAC .EQ. 1)WRITE $(3,2001)$ I2

$B M Y(I R)=B M Y(I R)+T 2 / 12$.

C --- $\star \pi \hbar \hbar \star$ MODULE TO COMPUTE THERMAL RESPONSE FUNCTION $\star * \hbar \hbar \star$

C (FT) AS FCT. OF CONVECTIVE COOLING (HCONV),

C WEARFLAT LENGTH (RLWORN) AND CUTTER RADIUS (RR)

2100 CONTINUE

$\operatorname{IF}(\operatorname{HR}(J)$. LE. HL (2) ) THEN

$\mathrm{IH}=2$

GO TO 2200

ENDIF

$\operatorname{IF}(\mathrm{HR}(\mathrm{J}), \mathrm{GE}$. HL ( 3 ) ) THEN

$\mathrm{IH}=4$

GO TO 2200

ENDIF

$I H=3$

2200 IF (WL (J) . LE.RL 50(2)) THEN

$\mathrm{IL}=2$

GO TO 2250

ENDIF 
$\mathrm{IL}=3$

2250 IHM=IH-1

$I L M=I L-1$

FSL=FT 50 ( ILM, IHM) + (HR ( J) -HL ( IHM) ) /

1 (HL (IH) $-\mathrm{HL}$ (IHM))*(FT50(ILM, IH) -FT50(ILM, IHM))

FLL $=$ FT $50($ IL, IHM $)+($ HR (J) $)-H L($ IHM $)) /$

1 (HL. (IH) -HL (IHM))*(FT 50 (IL, IH) -FT 50 (IL, IHM))

F $50=F S L+(W L(J)-R L 50($ ILM $)) /(R L 50($ IL $)-R L 50($ ILM $)) *(F L L-F S L)$

IF (WL (J) . LE . RL 75(2)) THEN

$I L=2$

GO TO 2350

ENDIF

IL $=3$

$\mathrm{IHM}=\mathrm{IH}-1$

ILM $=I L-1$

2350 FSL $=$ FT7 $5($ ILM, IHM $)+(\mathrm{HR}(\mathrm{J})-\mathrm{HL}(\mathrm{IHM}))$ )

1 (HL (IH) $-\mathrm{HL}($ IHM ))*(FT75(ILM, IH) - FT75(ILM, IHM))

FLL $=$ FT75 (IL, IHM $)+($ HR (J) $-\mathrm{HL}$ (IBM)) /

1 (HL (IH) -HL (IHM))*(FT75( IL , IH) -FT75(IL, IHM))

F75=FSL+(WL (J) -RL 75(ILM)) / (RL 75(IL) -RL75(ILM)) * (FLL-FSL)

$F T=F 50+(R R(J)-0.26) /(0.375-0.26) *(F 75-F 50)$

IF (FT. LE. O. ) THEN

WRITE (IDTO, 2400)

2400 FORMAT(' COMPUTED THERMAI RESPONSE FCT .LE. 0.1

1 ' PLEASE CHECK FOR INCORRECT CUTTER RADIUS OR CONV. COOLING'

2 ' COEFF.' /' CORRECT DATA AND RE-RUN')

CALL STOPP

ENDIF

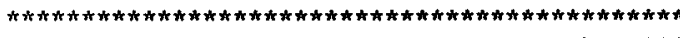

C -..- $* \pi \pi \pi \hbar$ CUTTER TEMPERATURE MODEL $* * * * \star$

2500 IF (AW(J) .EQ. 0.)GO TO 2600

IF (TMPFLG.EQ. 1 ) GO TO 2600

$\mathrm{T} 1=1.83 \mathrm{E}-3 * \mathrm{FC} * \mathrm{FP}(\mathrm{J}) \star \mathrm{FT} * \mathrm{XBC}(\mathrm{J}) \star \mathrm{RPM} / \mathrm{AW}(\mathrm{J})$

$T 2=1 .+0.0147 \star F T * R K \star(X B C(J) * R P M / R X / W L(J)) \star * .5$

$\mathrm{TW}(\mathrm{J})=(\mathrm{TFL}-32) / 1.8+.\mathrm{T} 1 / \mathrm{T} 2$

2550 GO TO 2650

$2600 \quad T W(J)=($ TFL -32.$) / 1.8$

2650 CONTINUE

IF (TW (J) GT . TWMAX (IR)) TWMAX (IR) $=\operatorname{TW}(\mathrm{J})$

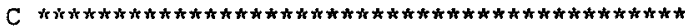

C --- $* * * * *$ CUTTER WEAR RATIO MODEL $* \hbar \star * * *$

$\mathrm{WR}(\mathrm{J})=\mathrm{FP}(\mathrm{J}) \star \mathrm{XBC}(\mathrm{J}) / \mathrm{FP}(1) / \mathrm{XBC}(1)$

IF (TW(J) .GT. 350.) THEN

$W R(J)=-W R(J)$

IT $1=-1$

ENDIF

$\operatorname{IF}(\operatorname{ABS}($ WR $(J)), G T \cdot A B S(W R M A X(I R))) W R M A X(I R)=W R(J)$

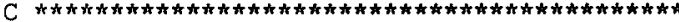

$\operatorname{IF}(\operatorname{ITRAC}$.EQ. 1)WRITE (3,308)XC1(J), ZC1(J),

$1 \mathrm{XC2}(\mathrm{J}), \mathrm{ZC2}(\mathrm{J})$

308 FORMAT(' FINAL: XCONT $1=$ ',F10.5,' 2 CONT1 $=$ ', F10.5,

1 ' $X$ CONT2 $=$ ',F10.5, ZCONT2=',F10.5)

C --- COMPUTE CUTTER RADIAL WEAR ANGLES

$\mathrm{DXC}=\mathrm{XC} 2(\mathrm{~J})-\mathrm{XC} 1(\mathrm{~J})$

IF (DXC.EQ. 0.)GO TO 1000

$W A(J)=A K^{\star A T A N}(-(Z C 2(J)-Z C 1(J)) / D X C)$

1000 CONTINUE

RETURN

END

SUBROUTINE CUTWEAR

PARAMETER (MXCUT $=48$ )

PARAMETER ( $T D=0.09$ )

$\mathrm{REAL} * 8 \mathrm{N4}$, N5

REAL*8 N1A, N2A, N1B, N2B

CHARACTER *20 ROCK

CHARACTER * 1 AIN

COMMON /AK/AK

COMMON /RESULTS /XW1 (MXCUT), XW2 (MXCUT), WL (MXCUT)

1 YOWORN (MXCUT), ITRAC, ZB (MXCUT), DE (MXCUT), NDE (MXCUT),

2 FP(MXCUT), WOB (5), BMX (5), BMY (5), FR(MXCUT), FV(MXCUT), FX(5)

3 FY (5), TORQ (5), TW(MXCUT), WR (MXCUT), FD (MXCUT)

4 WW(MXCUT), AW (MXCUT), IW1, XC1 (MXCUT), XC2 (MXCUT), ZC1 (MXCUT),

5 ZC2 (MXCUT), WA (MXCUT), WAO (MXCUT), ZW1 (MXCUT), ZW2 (MXCUT)

$6 \operatorname{TWMAX}(5), \operatorname{WRMAX}(5), \operatorname{PWNP}(M X C U T), 2 B C$ (MXCUT), XBC (MXCUT), IT1,

7 IC1, IC2, IC3, IC4, IC5, IC6, BF1, BF2, BF 3, BF 4, BF 5, ITYP (MXCUT), IP1, IP2 COMON /COMPOS/RP (MXCUT), AP (MXCUT), RR (MXCUT), HP (MXCUT)

1 BR (MXCUT) , MORD (MXCUT) , TNAI (MXCUT) , AI (MXCUT),

2 HPP (MXCUT); HR (MXCUT), BNOM

COMON /CTWEAR/AWMAX, NCMAX, ASUBWN, MODW, WRA (MXCUT),

1 FAMAX, XBCAMAX, DLH, C 6

COMMN /COMIOD/IDTO, IDTI, IDBD, IPRNT, IDWI, IDOC, IDWN

COMON /COMCON/NC, NRAY, HNOM, FEED RNOM, EPS, PEDGCT

COMMON /FT/FT5O $(3,4), \operatorname{FT75}(3,4), \operatorname{RL} 50(3), \operatorname{RL} 75(3), \operatorname{HL}(4)$, 


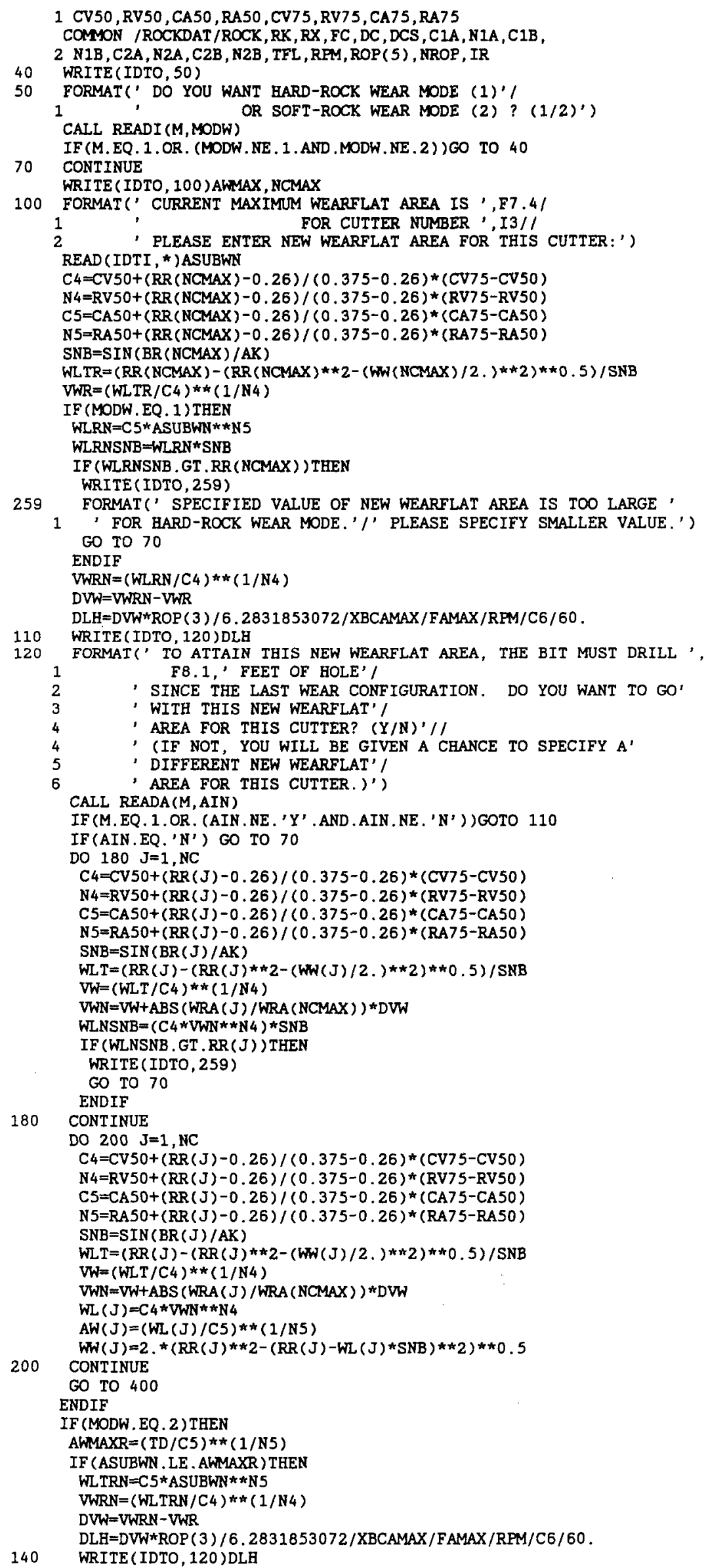




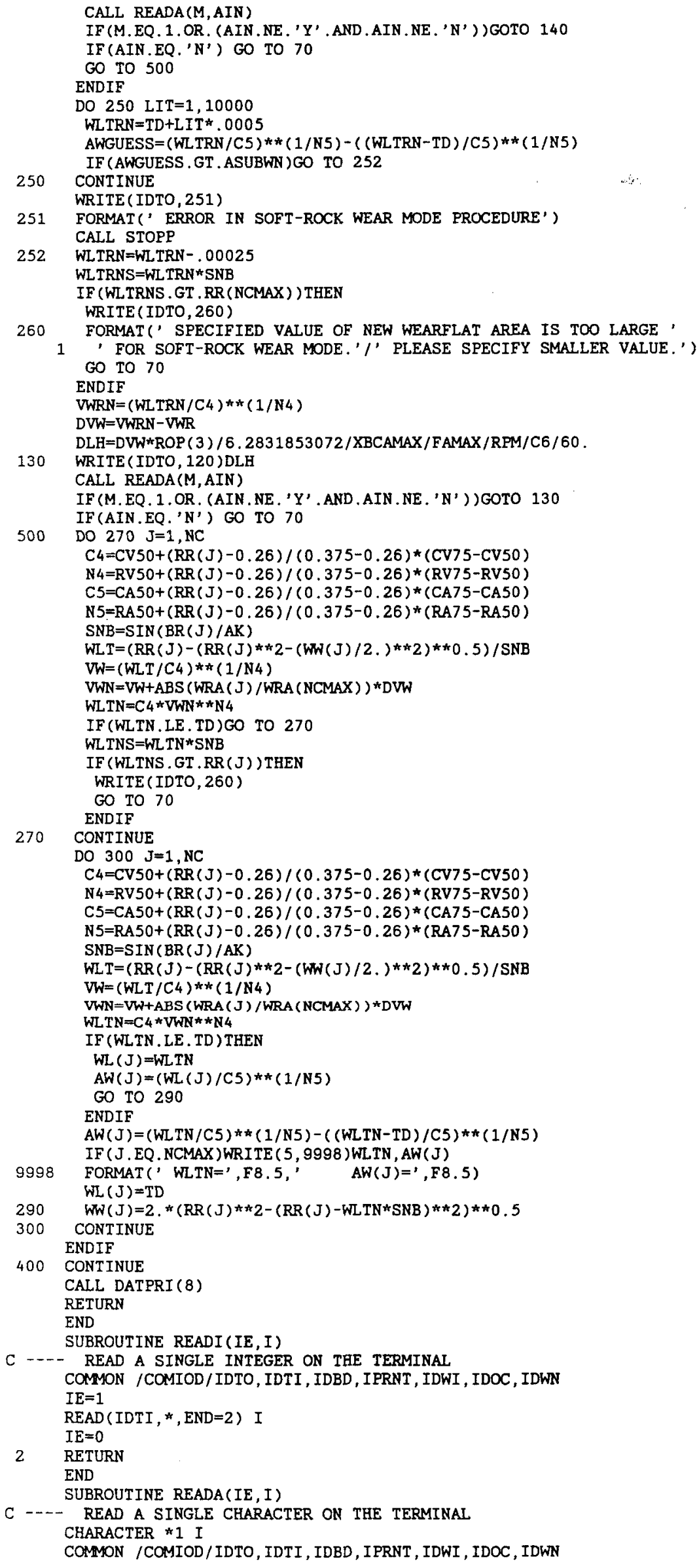




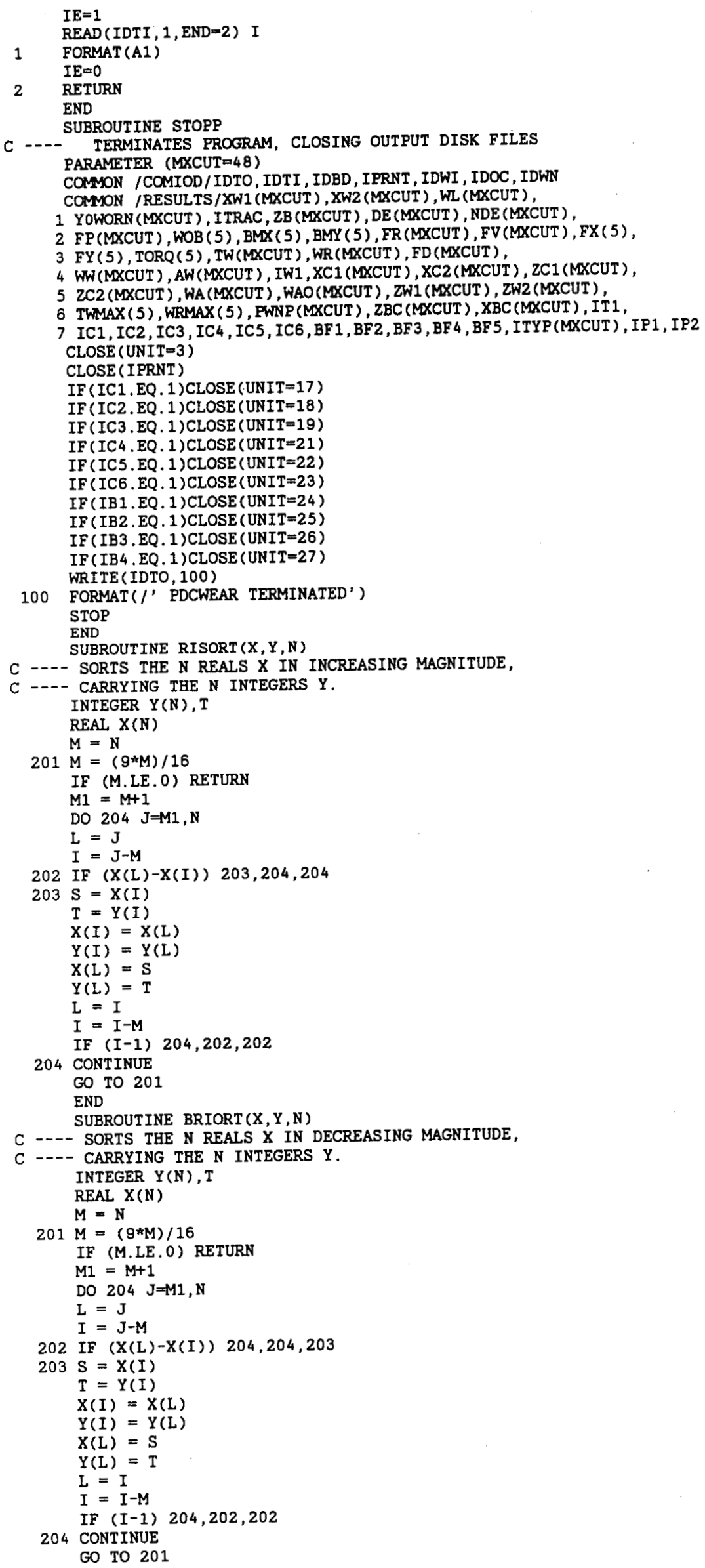


END

SUBROUTINE INPUT

C --- READS AND TESTS INPUT DATA

COMMN /COMIOD/IDTO, IDTI, IDBD, IPRNT, IDWI, IDOC, IDWN

COMON /COMCHA/IDATE, ITIME, BDNAME , RSNAME, WINAME, OCNAME

CHARACTER * 11 BDNAME, RSNAME, WINAME, OCNAME

CHARACTER * 10 IDATE, ITIME

CHARACTER *20 ROCK

PARAMETER (MXCUT=48)

REAL* 8 N1A, N2A, N1B, N2B

COMMON /AK/AK

COMMON /CTWEAR/AWMAX, NCMAX, ASUBWN, MODW, WRA (MXCUT) ,

1 FAMAX, XBCAMAX, DLH, C6

COMMON /COMPOS/RP(MXCUT), AP (MXCUT), RR (MXCUT), HP (MXCUT),

$1 \mathrm{BR}$ (MXCUT), MORD (MXCUT), TNAI (MXCUT), AI (MXCUT),

2 HPP(MXCUT), HR (MXCUT), BNOM

COMMN /COMRWK/VR (MXCUT), AR (MXCUT), C3 (MXCUT), D (MXCUT)

$1 \mathrm{E}$ (MXCUT), XMX (MXCUT), XMN (MXCUT), ZT (MXCUT), SRCX (MXCUT), WK (MXCUT)

COMMON /COMCON/NC, NRAY, HNOM, FEED, RNOM, EPS, PEDGCT

COMMON /RESULTS/XW1 (MXCUT), XW2 (MXCUT), WL (MXCUT),

1 YOWORN (MXCUT), ITRAC, ZB (MXCUT), DE (MXCUT), NDE (MXCUT)

2 FP(MXCUT), WOB (5), BMX (5), BMY (5), FR(MXCUT), FV(MXCUT), FX (5),

3 FY (5), TORQ (5), TW(MXCUT), WR (MXCUT), FD (MXCUT),

4 WW(MXCUT), AW(MXCUT), IW1, XC1 (MXCUT), XC2 (MXCUT), ZC1 (MXCUT),

5 ZC2 (MXCUT), WA (MXCUT), WAO (MXCUT), ZW1 (MXCUT), ZW2 (MXCUT),

6 TWMAX (5), WRMAX (5), PWNP (MXCUT), ZBC (MXCUT) , XBC (MXCUT) , IT 1,

7 IC1, IC2 , IC3, IC4 IC5, IC6, BF 1, BF 2, BF 3, BF 4, BF 5, ITYP (MXCUT), IP1, IP2

COMMON /ROCKDAT/ROCK, RK, RX, FC, DC, DCS , C1A, N1A;C1B,

2 N1B, C2A, N2A, C2B, N2B, TFL, RPM, ROP (5) , NROP, IR

CHARACTER * 1 AIN

C - - ENTER BIT DESIGN DATA FROM DISK

$\mathrm{IDT}=0$

MXCUT 1 $=$ MXCUT -1

10 WRITE(IDTO, 101)

101 FORMAT(/' ENTER NAME OF FILE CONTAINING BIT DESIGN DATA'/

1 ' ( 11 CAAR. OR LESS, INCLUDING .EXTENSION):')

READ (IDTI, ' (A11)', END=13) BDNAME

IF (BDNAME.EQ.' ') $G 0$ TO 10

OPEN (IDBD, FILE=BDNAME, STATUS=' OLD' )

REWIND IDBD

IDT $=1$

READ (IDBD , 102, END=200) RNOM, BNOM, HNOM

102 FORMAT ( $1 X, F 10.3,1 X, F 10.2,1 X, F 10.2)$

DO $11 \mathrm{NC}=1$, MXCUT1

READ (IDBD , 119, END=12)I, RP(NC), AP(NC), HPP(NC).

$1 \mathrm{AI}(\mathrm{NC}), \mathrm{RR}(\mathrm{NC}), \mathrm{BR}(\mathrm{NC}), \mathrm{HR}(\mathrm{NC})$

119 FORMAT (1X, I 3, 1X, F7.3, 1X, F7.3, 1X,F7.3,

1 1X,F7.3, 1X, F7.3, 1X, F7.3, 1X,F7.3)

11 CONTINUE

$\mathrm{NC}=\mathrm{MXCUT}$

READ ( IDBD , * , END=12)

GO TO 203

$12 \mathrm{NC}=\mathrm{NC}-1$

REWIND IDBD

CLOSE (IDBD, STATUS= ' KEEP')

13 IF (IDT.EQ.O) THEN

WRITE (IDTO, 108)

108 FORMAT (' INPUT DATA HAS NOT YET BEEN ENTERED') GO TO 10

ENDIF

IF (NC. GT.MXCUT1)GO TO 203

C --- WANT TO LIST INPUT ON TERM. ?

15 WRITE (IDTO, 104)NC

104 FORMAT (/' THERE ARE' I4 ' CUTTERS'/

1 'DO YOU WISH TO LIST THE BIT DESIGN INPUT'

2 ' DATA ON THE TERMINAL? $\left.(\mathrm{Y}, \mathrm{N})^{\prime}\right)$

CALL READA (M, AIN)

IF (M.EQ. 1. OR. (AIN NE 'Y', AND.AIN.NE.'N')) GO TO 15

IF (AIN.EQ. ' $Y$ ') CALL DATPRI(1)

C --- TEST BIT DESIGN DATA

40 DO $41 \mathrm{~J}=2$, NC

$\operatorname{IF}(\operatorname{RP}(J) . \mathrm{LT} \cdot \operatorname{RP}(\mathrm{J}-1))$ )O TO 42

41 CONTINUE

C DATA IS IN ORDER

GO TO 45

$C$ DATA IS OUT OF ORDER

42 WRITE(IDTO, 118)

118 FORMAT(' CUTTERS ARE NOT ORDERED IN ASCENDING'

1 ' RADIAL POSITION' )

WRITE (IDTO, 114)

114 FORMAT(' PLEASE CORRECT INPUT DATA FILE(S) AND RE-RUN')

CALL STOPP

45 IF (RNOM.LE. O.) THEN 


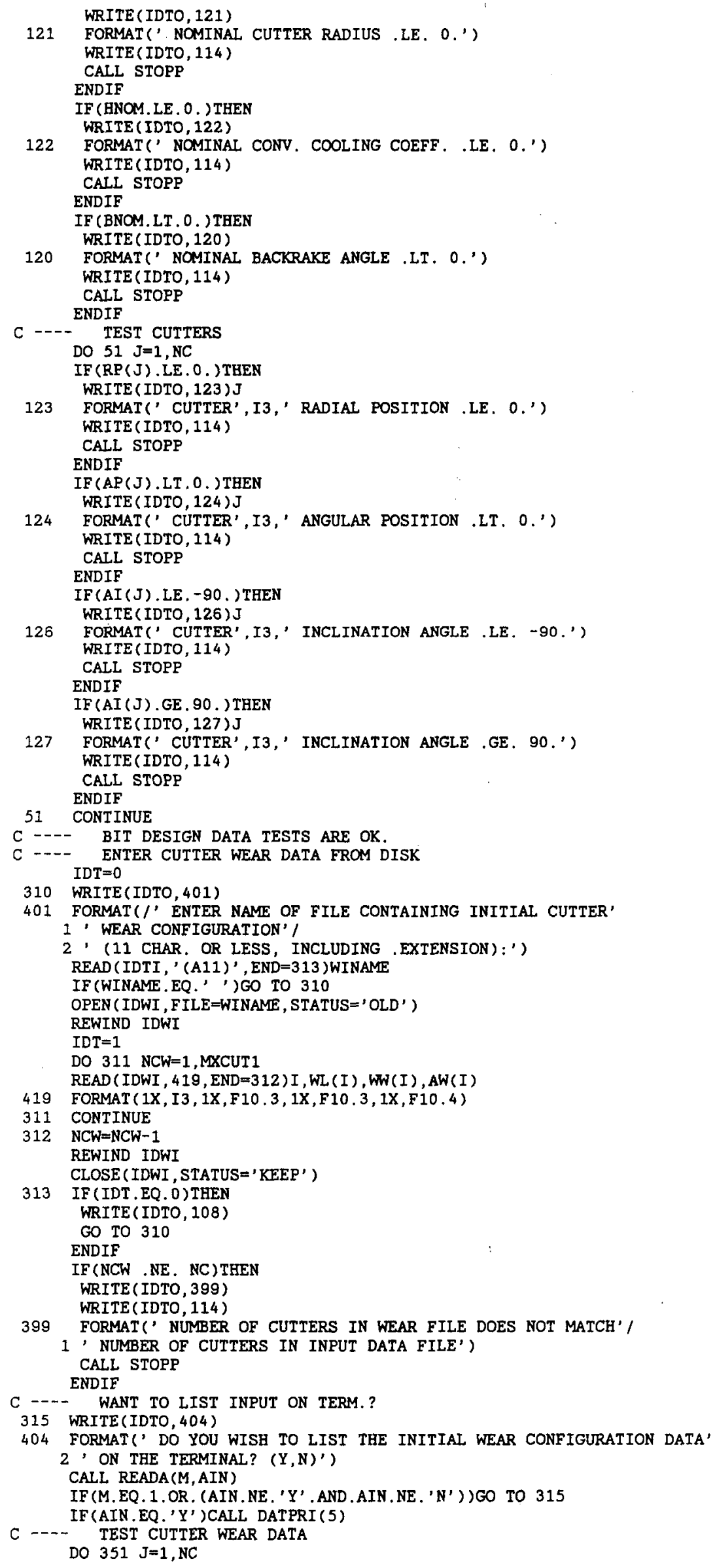




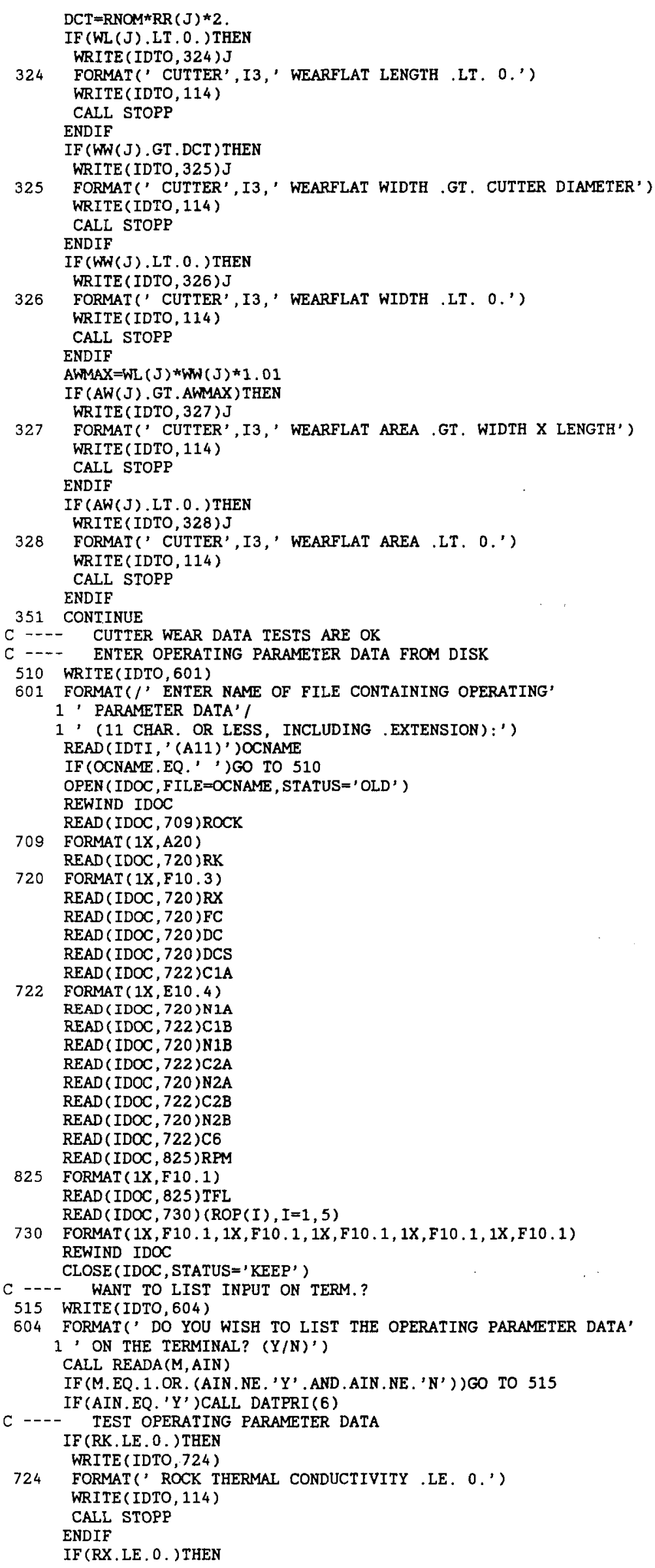


WRITE (IDTO, 725)

725 FORMAT(' ROCK THERMAL DIFFUSIVITY .LE. 0.')

WRITE (IDTO, 114)

CALL STOPP

ENDIF

IF (FC. LE. O. ) THEN

WRITE (IDTO, 726)

726 FORMAT (' ROCK-CUTTER FRICTION COEFFICIENT .LE. 0.')

WRITE (IDTO, 114)

CALL STOPP

ENDIF

IF (DC.LE. O.) THEN

WRTTE (IDTO, 727)

727 FORMAT(' WORN CUTTER DRAG COEFFICIENT .LE. 0.')

WRITE (IDTO, 114)

CALL STOPP

ENDIF

IF (DCS.LE.0.)THEN

WRITE (IDTO, 767)

767 FORMAT(' SHARP CUTTER DRAG COEFFICIENT .LE. O.')

WRITE (IDTO, 114)

CALL STOPP

ENDIF

IF (C1A.LE . 0.)THEN

WRITE (IDTO, 728)

728 FORMAT(' WORN TYPE A CUTTER CORRELATION CONSTANT C1 .IE. O.') WRITE (IDTO, 114)

CALL STOPP

ENDIF

IF (N1A . LE . O. )THEN

WRITE (IDTO, 729)

729 FORMAT(' WORN TYPE A CUTTER CORRELATION EXPONENT N1 .LE. O.') WRITE (IDTO, 114)

CALL STOPP

ENDIF

IF (C1B. LT . O. )THEN

WRITE (IDTO, 828)

828 FORMAT(' WORN TYPE B CUTTER CORRELATION CONSTANT C1 .LT. $0 . '$ ) WRITE (IDTO, 114)

CAIL STOPP

ENDIF

IF (N1B.IT. 0.)THEN

WRITE (IDTO, 829)

829 FORMAT(' WORN TYPE B CUTTER CORRELATION EXPONENT N1 .LT. 0.') WRITE (IDTO, 114)

CALL STOPP

ENDIF

IF (C2A.LE. O.) THEN

WRITE (IDTO, 758)

758 FORMAT(' SHARP TYPE A CUTTER CORRELATION CONSTANT C2 .LE. 0.') WRITE ( IDTO, 114)

CALL STOPP

ENDTF

IF (N2A.LE. 0.) THEN

WRITE (IDTO, 759)

759 FORMAT(' SHARP TYPE A CUTTER CORRELATION EXPONENT N2 .LE. 0.') WRITE ( IDTO, 114)

CALL STOPP

ENDIF

IF (C2B.LT. 0.) THE

WRITE(IDTO, 858)

858 FORMAT(' SHARP TYPE B CUTTER CORRELATION CONSTANT C2 .LT. 0.') WRITE (IDTO, 114)

CALL STOPP

ENDIF

IF (N2B . LT . 0.) THEN

WRITE (IDTO, 859)

859 FORMAT(' SHARP TYPE B CUTTER CORRELATION EXPONENT N2 .LT. O.') WRITE (IDTO, 114)

CALI STOPP

ENDIF

IF (C6. LE. 0. ) THEN

WRITE (IDTO, 860)

860 FORMAT(' ABRASIVE WEAR CONSTANT C6 .LE. 0.')

WRITE (IDTO, 114)

CALL STOPP

ENDIF

IF (RPM. LE. O. ) THE

WRITE (IDTO, 731)

731 FORMAT(' BIT ROTARY SPEED .LE. O.')

WRITE (IDTO, 114)

CALL STOPP

ENDIF 


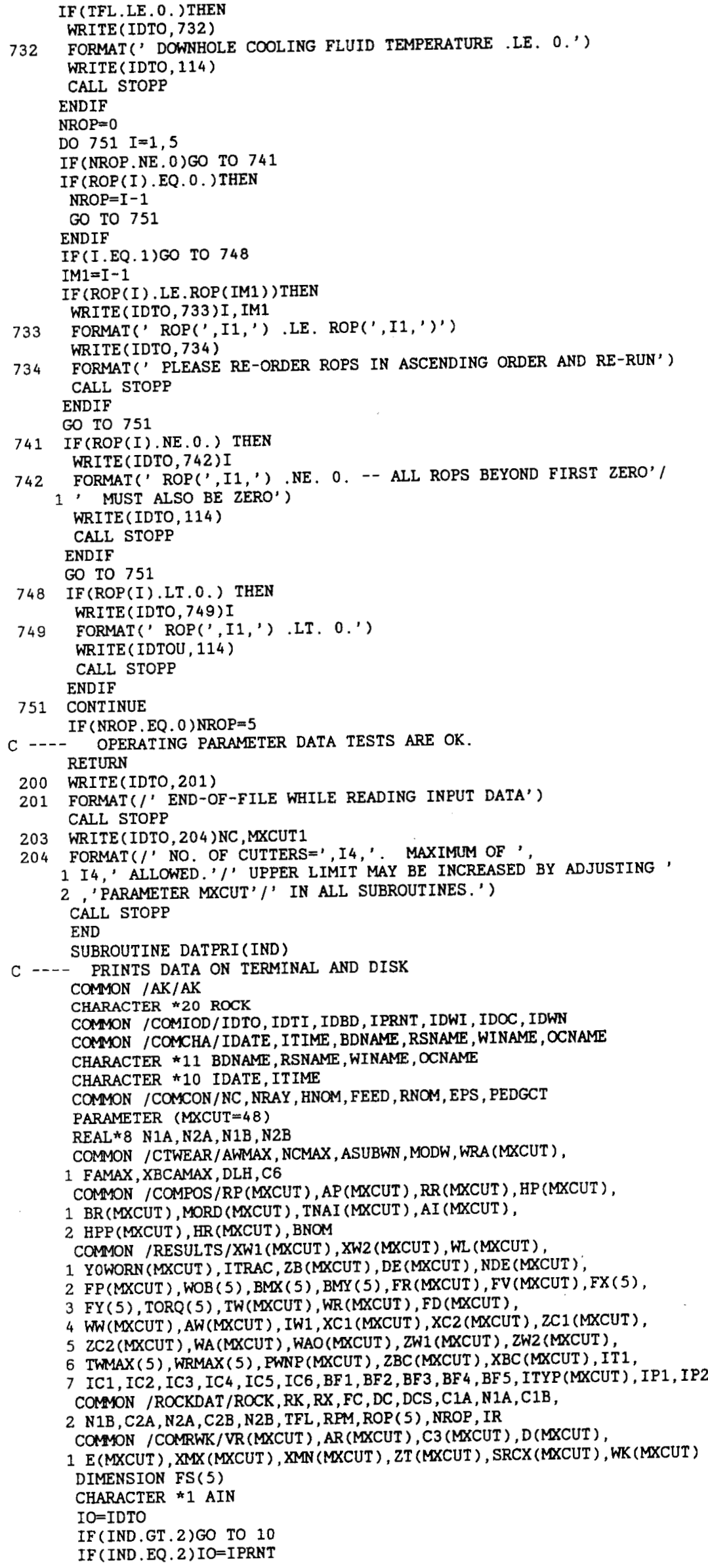


$\mathrm{HL}=0$.

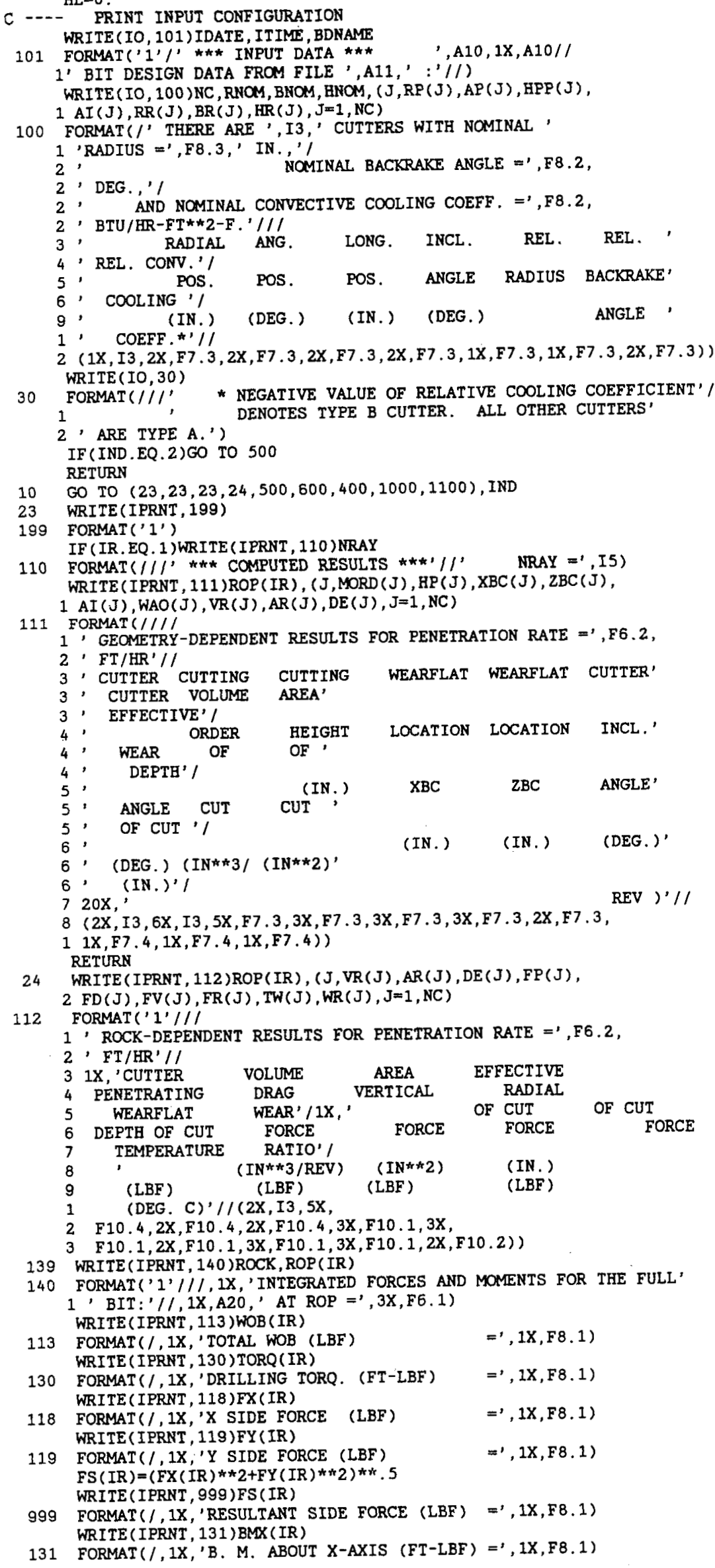


WRITE (IPRNT, 132) BMY (IR)

132 FORMAT $(/, 1 X, ' B$. M. ABOUT Y-AXIS (FT-LBF) $=\prime, 1 X, F 8.1)$

WRITE (IO, 441) TWMAX (IR)

WRITE (IO, 442) WRMAX (IR)

GO TO $(250,255,260,265,270)$, IR

250 WRITE (IO, 440)ROCK, ROP (1)

WRITE (IO, 413 )WOB (1)

WRITE (IO, 430) TORQ (1)

WRITE (IO, 418)EX(1)

WRITE (I0, 419)FY (1)

WRITE (IO, 499)FS(1)

WRITE (IO, 431)BMX (1)

WRITE (IO, 432)BMY (1)

WRITE (IO, 441) TWMAX (1)

WRITE (IO, 442 ) WRMAX (1)

GO TO 300

255 WRITE (IO, 440)ROCK, ROP (1), ROP (2)

WRITE (IO, 413)WOB (1), WOB (2)

WRITE (IO, 430) TORQ (1), TORQ (2)

WRITE (IO, 418)FX(1), FX(2)

WRITE (IO, 419)FY(1), FY (2)

WRITE (IO, 499)FS(1), FS(2)

WRITE (IO, 431) BMX (1), BMX (2)

WRITE ( IO 432) BMY (1), BMY (2)

WRITE (IO, 441) TWMAX (1), TWMAX (2)

WRITE (IO, 442 ) WRMAX (1), WRMAX (2)

GO TO 300

260 WRITE (IO, 440)ROCK, ROP (1), ROP (2), ROP (3)

WRITE (IO, 413) WOB (1), WOB (2), WOB (3)

WRITE ( IO, 430) TORQ (1), TORQ (2), TORQ (3)

WRITE (IO, 418) FX(1), FX(2), FX (3)

WRITE (IO, 419) FY (1), FY (2), FY (3)

WRITE(IO, 499)FS(1), FS (2), FS (3)

WRITE (I0, 431) BMX (1), BMX (2), BMX (3)

WRITE (IO, 432)BMY (1), BMY (2), BMY (3)

WRITE (IO, 441) TWMAX (1), TWMAX (2), TWMAX (3)

WRITE ( IO , 442) WRMAX (1), WRMAX (2), WRMAX (3)

GO TO 300

$265 \operatorname{WRITE}(10,440) \operatorname{ROCK}, \operatorname{ROP}(1), \operatorname{ROP}(2), \operatorname{ROP}(3), \operatorname{ROP}(4)$

WRITE (IO, 413) WOB (1), WOB (2), WOB (3), WOB (4)

WRITE (IO, 430)TORQ (1), TORQ (2), TORQ (3), TORQ(4)

WRITE (IO, 418)FX(1), FX(2), FX(3), FX(4)

WRITE (IO, 419)FY(1),FY(2),FY(3),FY(4)

WRITE (IO, 499)FS(1), FS(2), FS (3), FS (4)

WRITE (IO, 431) BMX (1), BMX (2), BMX (3), BMX (4)

WRITE (IO , 432) BMY (1), BMY (2), BMY (3), BMY (4)

WRITE (I0, 441) TWMAX (1), TWMAX (2), TWMAX (3), TWMAX (4)

WRITE ( IO, 442) WRMAX (1), WRMAX (2), WRMAX (3), WRMAX (4)

GO TO 300

270 WRITE (IO, 440)ROCK, ROP (1), ROP (2), ROP (3), ROP (4), ROP (5)

WRITE (IO , 413) WOB (1), WOB (2), WOB (3), WOB (4), WOB (5)

WRITE (IO, 430) TORQ (1), TORQ (2), TORQ (3), TORQ (4), TORQ ( 5 )

WRITE (IO, 418)FX(1), FX(2), FX(3), FX(4), FX(5)

WRITE (IO, 419)FY(1),FY(2),FY(3),FY(4),FY(5)

WRITE (IO, 499)FS(1), FS(2), FS (3), FS (4), FS ( 5$)$

WRITE (IO, 431) BMX (1), BMX (2), BMX (3), BMX (4), BMX (5)

WRITE (IO, 432) BMY (1), BMY (2), BMY (3), BMY (4), BMY (5)

WRITE (I0, 441) TWMAX (1), TWMAX (2), TWMAX (3), TWMAX (4), TWMAX (5)

WRITE (IO , 442)WRMAX (1), WRMAX (2), WRMAX (3), WRMAX (4), WRMAX (5)

300 IF (IP1.EQ.0) THEN

$I P 1=I P 1+1$

WRITE (IDTO, 310)

310 FORMAT( $/$ ' DO YOU WANT TO SEND DATA TO CUTTER PLOT FILES? (Y/N)')

CALL READA (M, AIN)

IF (M .EQ. 1 .OR. (AIN.NE. 'Y' .AND. AIN.NE. 'N') )GOTO 300

IF (AIN.NE . 'Y') GOTO 320

700 WRITE(IDTO, 710)

710 FORMAT ( $/$ ' PENETRATING FORCE VS. CUTTER NUMBER? (Y/N)')

CALL READA(M, AIN)

IF (M .EQ. 1 .OR. (AIN.NE. 'Y' .AND. AIN.NE. 'N') ) GOTO 700

IF (AIN.EQ. 'Y') THEN

$I C 1=1$

OPEN (UNIT=17, NAME=' FVSCN . DAT', TYPE= 'NEW' )

ENDIF

715 WRITE(IDTO, 720)

720 FORMAT ( /' EFFECTIVE DEPTH OF CUT VS. CUTTER NUMBER? (Y/N)') CALL READA(M,AIN)

IF (M EQ 1 OR (AIN NE 'Y' AND AIN .NE 'N')) GOTO 715

IF (AIN.EQ. ' $Y$ ') THEN

IC $2=1$

OPEN (UNI $T=18, \mathrm{NAME}=$ ' DEFVSCN . DAT' , TYPE='NEW' )

ENDIF 
FORMAT ( /' WEARFLAT TEMPERATURE VS. CUTTER NUMBER? (Y/N)')

CAIL READA (M, AIN)

IF (M .EQ. 1 , OR. (AIN.NE. 'Y' . AND.AIN.NE.' 'N')) COTO 725

IF (AIN. EQ. ' $Y$ ') TEEN

$\mathrm{IC} 3=1$

OPEN (UNIT=19, NAME='TWVSCN DAT', TYPE='NEW')

ENDIF

735 WRITE(IDTO, 740)

740 FORMAT (/' WEAR RATIO VS. CUTTER NUMBER? ( $Y / N)$ ')

CALL READA (M,AIN)

IF (M .EQ. 1 .OR. (AIN.NE. 'Y' AND.AIN.NE. 'N')) GOTO 735

IF (AIN.EQ. ' $Y$ ') THEN

IC $4=1$

OPEN (UNIT $=21$, NAME = ' WRVSCN .DAT', TYPE='NEW')

ENDIF

745 WRITE (IDTO, 750)

750 FORMAT (/' ROCK VOLUME VS. CUTTER NUMBER? (Y/N)')

CALL READA (M, AIN)

IF (M .EQ. 1 .OR. (AIR. NE. 'Y' . AND. AIN. NE. 'N') ) GOTO 745

IF (AIN.EQ. ' $Y$ ') THEN

$I C 5=1$

OPEN (UNI T $=22$, NAME = ' VRVSCN. DAT', TYPE = 'NEW' )

ENDIF

755 WRITE(IDTO, 760)

760 FORMAT ( /' AREA OF CUT VS. CUTTER NUMBER? (Y/N)')

CALL READA(M,AIN)

IF (M .EQ. 1 .OR. (AIN.NE. 'Y' . AND. AIN.NE. 'N')) GOTO 755

IF (AIN, EQ. 'Y') THEN

$I C 6=1$

OPEN (UNIT $=23$, NAME=' ARVSCN . DAT' , TYPE='NEW' )

ENDIF

ENDIF

IF (IC1 EQ . 1)WRITE $(17,217)$ BDNAME, WINAME, OCNAME, ROP (IR)

IF (IC2 EO 1)WRITE $(18,217)$ BDNAME WINAME OCNAME, ROP (IR)

IF (IC3.EQ . 1) WRITE (19, 217 ) BDNAME, WINAME, OCNAME , ROP (IR)

IF (IC4.EQ. 1) WRITE (21, 217 )BDNAME, WINAME, OCNAME, ROP (IR)

IF (IC5 . EQ. 1) WRITE (22, 217) BDNAME, WINAME, OCNAME , ROP (IR)

IF (IC6 . EQ . 1) WRITE $(23,217$ ) BDNAME, WINAME, OCNAME , ROP (IR)

217 FORMAT (A11/,A11/,A11/,F7.2)

DO $115 \mathrm{~J}=1$, NC

IF (IC1.EQ. 1)WRITE $(17,117) \mathrm{J}, \mathrm{FP}(\mathrm{J})$

IF (IC2.EQ. 1)WRITE $(18,117) \mathrm{J}, \mathrm{DE}(\mathrm{J})$

IF (IC3.EQ. 1)WRITE (19,117) J, TW(J)

IF (IC4 . EQ . 1)WRITE $(21,117) \mathrm{J}$, WR (J)

IF (IC5.EQ.1)WRITE $(22,117) \mathrm{J}, \operatorname{VR}(\mathrm{J})$

IF (ICG. EQ.1)WRITE (23,117) J,AR(J)

115 CONTINUE

117 FORMAT(I2,', ', F12.4)

320 CONTINUE

RETURN

400 CONTINUE

439 WRITE (IO, 440 )ROCK, $\operatorname{ROP}(1), \operatorname{ROP}(2), \operatorname{ROP}(3), \operatorname{ROP}(4), \operatorname{ROP}(5)$

440 FORMAT (' 1 ' $/ 1 \%$ ' INTEGRATED AND SUMMARY DATA FOR THE FULL BIT'/

1 ' AT ALL SPECIFIED PENETRATION RATES * :'/1

$21 \mathrm{X}, \mathrm{A} 20 \mathrm{,}^{\prime}$ 'AT ROP $=$ ', 3X,F6.1,3X,F6.1,3X,F6.1, 3X,F6.1,

$33 \mathrm{X}, \mathrm{F} 6.1 \mathrm{f}^{\prime}$

4.

WR $\overline{I T E}(I 0,413)$ WOB (1), WOB (2), WOB (3), WOB (4), WOB (5)

413 FORMAT $(1,1 \mathrm{X}$, 'TOTAL WOB (LBF) $=$, IX,F8.1,

1 1X,F8,1,1X,F8.1, 1X,F8,1, 1X,F8.1)

WRITE (IO, 430) TORQ (1), TORQ (2), TORQ (3), TORQ (4), TORQ ( 5 )

430 FORMAT(IX,'DRILLING TORQ. (FT-LBF) =', 1X,F8.1,

1 1X,F8.1, 1X,F8.1, 1X,F8.1, 1X,F8.1)

WRITE (IO, 418) FX(1), FX(2), FX(3), FX(4), FX(5)

418 FORMAT(/,1X,'X SIDE FORCE (LBF) =', $1 X, F 8.1$,

1 1X,F8.1.1X,F8.1, 1X,F8.1, 1X,F8.1)

WRITE(IO, 419)FY(1), FY(2),FY(3), FY (4), FY(5)

419 FORMAT ( $1 X, ' Y$ SIDE FORCE (LBF)

$1 \quad 1 X, F 8.1,1 X, F 8.1,1 X, F 8.1,1 X, F 8.1)$

WRITE (IO 499)FS(1), FS(2), FS(3), FS (4), FS (5)

499 FORMAT ( $1 X$, 'RESULTANT SIDE FORCE (LBF) =', 1X,F8.1

$1 \quad 1 X, F 8.1,1 X, F 8.1,1 X, F 8.1,1 X, F 8.1)$

WRITE (IO 431) BMX (1), BMX (2), BMX (3), BMX (4), BMX (5)

431 FORMAT $(1,1 \mathrm{X}, \mathrm{B}, \mathrm{M}$. ABOUT X-AXIS (FT-LBF) $=$ ', $1 \mathrm{X}, F 8.1$

$1 \quad 1 X, F 8.1,1 X, F 8.1,1 X, F 8,1,1 X, F 8.1)$

WRITE (IO 432) BMY (1), BMY (2), BMY (3), BMY (4), BMY (5)

432 FORMAT ( $1 X, ' B$. M. ABOUT $Y$-AXIS (FT-LBF) =', $1 X, F 6,1$,

$1 \quad 1 X, F 8.1,1 X, F 8.1,1 X, F 8.1,1 X, F 8.1)$

WRI TE (IO, 441) TWMAX (1), TWMAX (2), TWMAX (3), TWMAX (4), TWMAX (5)

441 FORMAT ( ) 1X, 'MAX. WEARFLAT TEMP. (DEG. C)=', 1X,F8.1

1 1X,F8.1, 1X,F8.1, 1X, F8.1,1X,F8.1)

WRITE (IO 4 42) WRMAX (1), WRMAX (2), WRMAX (3), WRMAX (4), WRMAX (5)

FORMAT ( $1 X$, 'MAX. WEAR RATIO

$=$, IX,F8.2, 


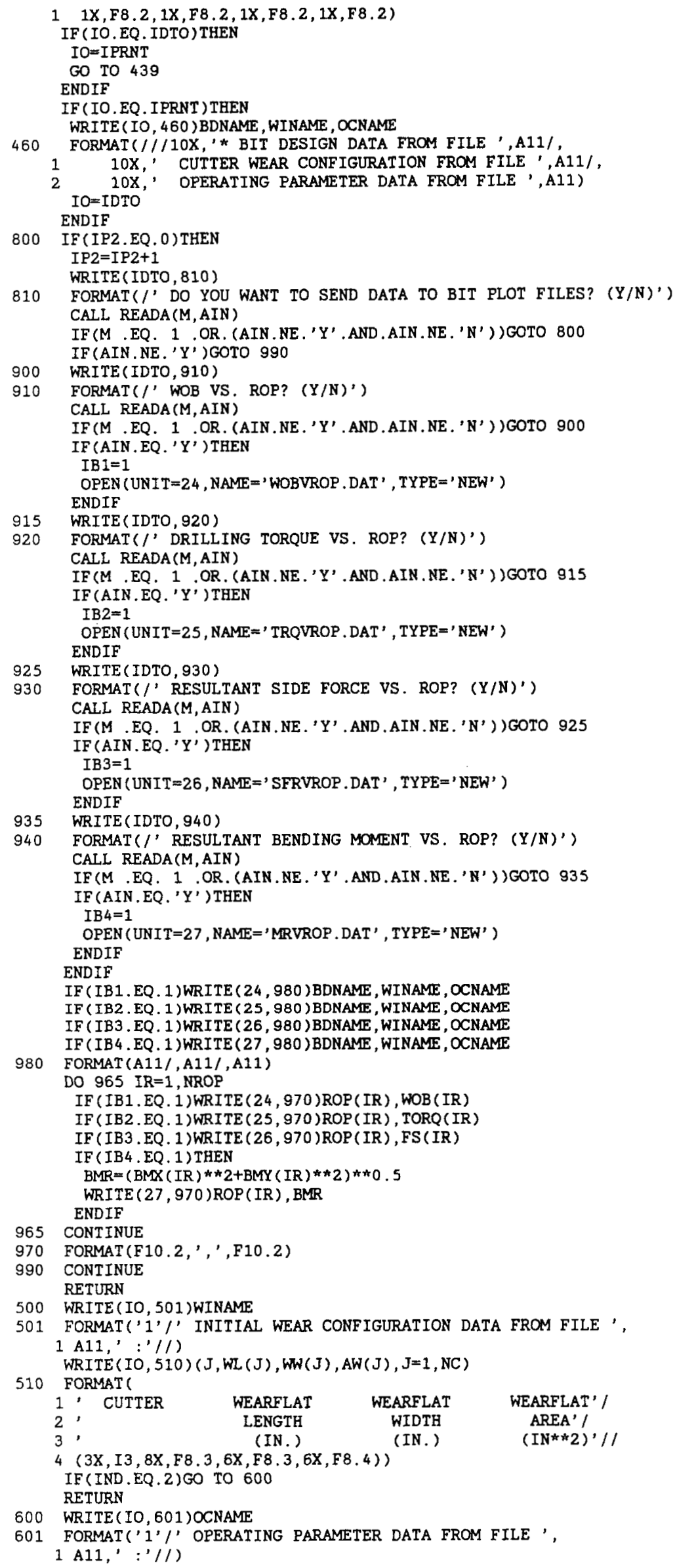




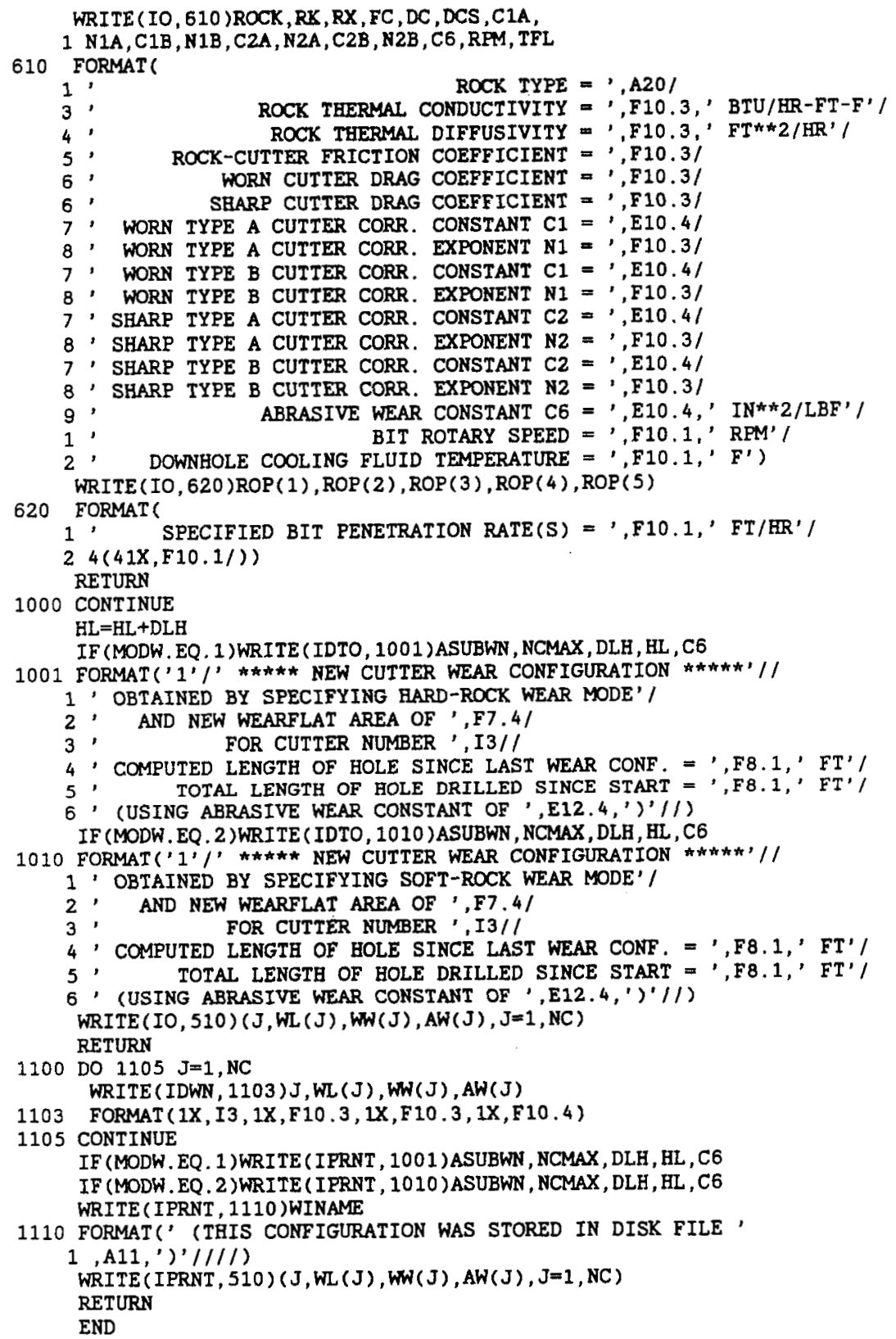


APPENDIX C

THERMAL NUMERICAL MODELING OF A 0.75 -INCH PDC CUTTER 


\section{APPENDIX C \\ THERMAL NUMERICAL MODELING OF A $0.75-$ INCH PDC CUTTER}

Our earlier work $[22,26,27]$ presented thermal modeling results for PDC cutters with 0.50 -inch diameter compacts. Because of the availability of cutters with larger compacts and because of the apparent increased efficiency associated with larger cutters, the models of the earlier work were modified to represent cutters using 0.75 -inch diameter compacts.

Three wear configurations were used in this study: mildly worn ( $L=0.0266$ inch), moderately worn ( $\mathrm{L}=0.096$ inch), and severely worn ( $\mathrm{L}=0.148$ inch) (see Figure 29). In the mildly worn case, the wearflat extends only across the diamond layer; hence, $L=t_{d} / \cos \beta$. A diamond thickness of 0.025 inch was assumed. In the moderately and severely worn cases, the wearflat extends into the WC-Co compact to which the PDC layer is bonded. The length of the wearflat for each wear configuration is shown in Table C-1 on the next page. The values used for the thermal properties of the cutter materials were the same as those used in the baseline case of our earlier work [27].

Both fully exposed cutters and cutters recessed into the bit body were considered. With the fully exposed cutters, convective cooling boundary conditions were applied to all exposed cutter and bit surfaces. With the recessed cutters, convective cooling along the diamond face was restricted to the lower half of the face, and no convective cooling was provided to the top surfaces of the cutter compact. All surfaces not shown as being convectively cooled in Figure 29 were given an insulating boundary condition. A fixed, uniform heat flux, $q$, was applied as a boundary condition at the cutter wearflat for a variety of convective cooling coefficients ranging from 17.61 to $1.761 \times 10^{4} \mathrm{Btu} / \mathrm{hr} \mathrm{ft}^{2} \mathrm{~F}\left(0.01\right.$ to $\left.10.0 \mathrm{~W} / \mathrm{cm}^{2}{ }^{\circ} \mathrm{C}\right)$.

As in the earlier studies, a general purpose finite element thermal computer code, COYOTE [50], was used to compute temperature distributions in the cutters. The average temperature computed for the surface node points along the wearflat, $\bar{T}_{w}$, was reduced by the assumed cooling fluid temperature, $\mathrm{T}_{f}$, and divided by the ${ }^{\mathrm{w}}$ input heat flux to obtain the thermal response of the cutter:

$$
f=\frac{\bar{T}_{w}-T_{f}}{q}
$$

Values of $f$ are shown in Table $C-1$ as a function of the assumed cooling coefficients for both the recessed and fully exposed cutters. Also shown for comparison purposes are the results obtained in Ref. 27 for cutters with a 0.5 -inch diameter compact. 
TABLE $C-1$

COMPUTED THERMAL RESPONSE FUNCTION FOR 0.75-INCH AND 0.5-INCH CUTTERS

Thermal Response Function, $f\left({ }^{\circ} \mathrm{C} \mathrm{cm}{ }^{2} / \mathrm{W}\right)$

Mild1y Worn $(L=0.027) \quad$ Mod. Worn $(L=0.096) \quad$ Sev. Worn $(L=0.148)$

$\mathrm{h}\left(\mathrm{Btu} / \mathrm{hr} \mathrm{ft}^{2} \mathrm{o}_{\mathrm{F}}\right) \quad \mathrm{h}\left(\mathrm{Btu} / \mathrm{hr} \mathrm{ft}^{2}{ }^{\mathrm{o}} \mathrm{F}\right) \quad \mathrm{h}\left(\mathrm{Btu} / \mathrm{hr} \mathrm{ft}^{2} \mathrm{o}_{\mathrm{F}}\right)$

CF* $\quad 17.61 \quad 176.1 \quad 1761 \quad 17610 \quad 17.61 \quad 176.1 \quad 1761 \quad 17610.17 .61 \quad 176.1 \quad 1761 \quad 17610$

\begin{tabular}{lrrrrrrrrrrrr}
1 & .830 & .174 & .067 & .026 & 3.003 & .609 & .234 & .106 & 4.653 & .913 & .345 & .166 \\
2 & & & .064 & & & & & .224 & & & & \\
3 & .956 & .170 & .064 & .026 & & & & & 5.459 & .896 & .325 & .166 \\
\hline
\end{tabular}

$* \mathrm{CF}=$ cutter configuration

$1=0.75$-inch compact cutter recessed

$2=0.75$-inch compact cutter fully exposed

$3=0.50$-inch compact cutter fully exposed (Ref. 27). The length of the "severely worn" wearflat of the 0.75 -inch cutter is the same as the length of the "moderately worn" wearflat of the 0.5-inch cutter in Ref. 27. Wearflat length $L$ is in inches.

Note that with the moderate cooling coefficients, the thermal response function for the recessed 0.75 -inch cutter is slightly larger than that for the 0.5-inch cutter in cases where a direct comparison is possible. This is caused by the larger mean distance for heat to flow from the wearflat to a cooled surface. For the lower cooling coefficients, however, the thermal response function for the recessed 0.75 -inch cutter is lower than that for the 0.5 -inch cutter. This is because at very low convective cooling rates, heat transfer is controlled by convection, not conduction, so the larger cooled surface area of the 0.75 -inch cutter results in a cooler wearflat. In any case, the difference in thermal response function between the different size cutters is not significant.

The effects of fully exposing the 0.75-inch cutter appear to also be insignificant in that the thermal response function at a given convective cooling coefficient is approximately the same as that for the recessed 0.75inch cutter. This is due to the fact that most of the heat is convected from the lower half of the diamond face, and cooling the upper half of the face does little to further cool the wearflat. It should be noted, however, that by fully exposing the cutter, the standoff distance between the bit body and the rock surface is increased, thereby reducing fluid velocities and convective cooling coefficients. As a result, a bit with fully exposed 0.75inch cutters could run significantly hotter than a bit with recessed 0.75 -inch cutters at the same drilling fluid flow rate. For this reason, the results for the recessed 0.75 -inch cutters are incorporated into PDCWEAR. 


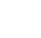$$
\text { - }
$$ 
APPENDIX D

PDCWEAR INPUT GUIDE 


\section{APPENDIX D}

PDCWEAR INPUT GUIDE

Three input files are required to run PDCWEAR. These files contain the bit design data, the initial cutter wear configurations, and the operating conditions. The names chosen for these files may be 1 to 11 alphanumeric characters in length, including the file extension. The utility program, FORMAT, may be run interactively by the user to properly format input parameter values and create the required input files. FORMAT produces three files suitable for input to PDCWEAR. These names of these files are selected by the user. A listing of FORMAT is provided in Appendix E.

\section{Bit Design Data File}

This file contains data describing the sizes of cutters, their locations in three-dimensional space, their backrake angles, and their convective cooling coefficients. Data is read into PDCWEAR in the following format:

READ ( , 102) RNOM , BNOM , HNOM

102 FORMAT (1X, F10.3, IX, F10.2, 1X, F10.2)

$\operatorname{READ}(, 119)(\mathrm{J}, \mathrm{RP}(\mathrm{J}), \operatorname{AP}(\mathrm{J}), \mathrm{HHP}(\mathrm{J}), \operatorname{AI}(\mathrm{J}), \operatorname{RR}(\mathrm{J}), \mathrm{BR}(\mathrm{J}), \mathrm{HR}(\mathrm{J}), \mathrm{J}=1, \mathrm{NC})$

119 FORMAT $(1 X, I 3,7(1 X, F 7.3))$

where:

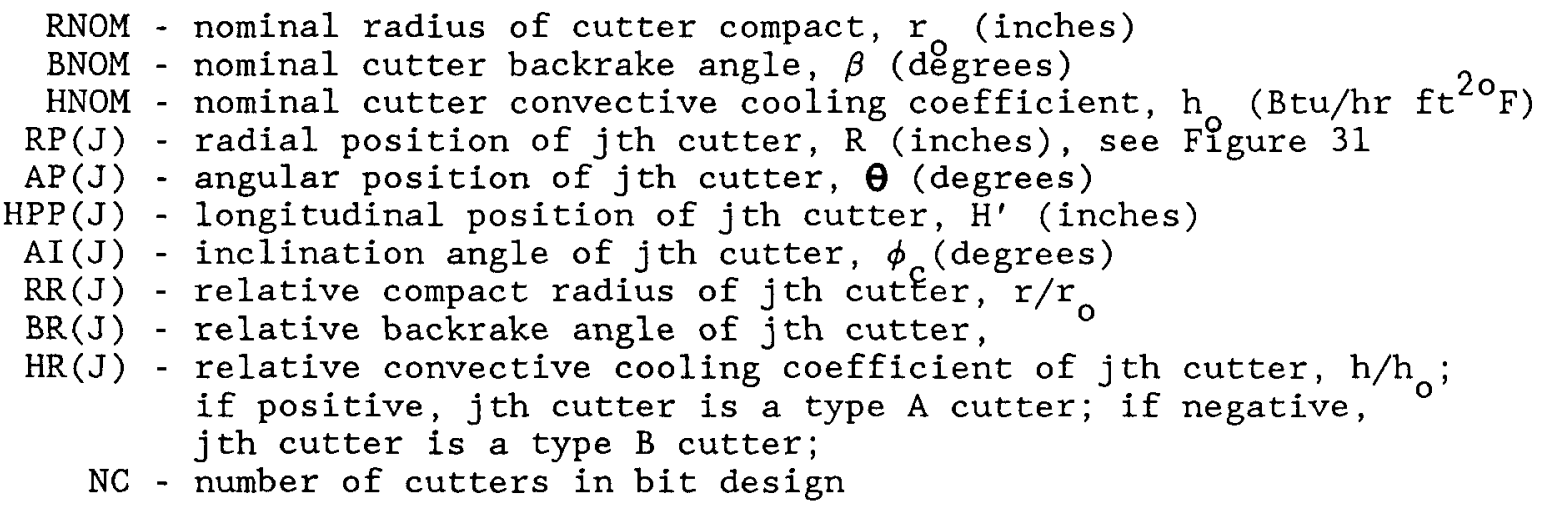

Some of these parameters are illustrated in Figure 31.

The radius of the cutter compact affects the cutter profile and thus the effective depth of cut and cutter forces. The compact radius traditionally used in PDC bits is approximately 0.25 inches (0.50-inch diameter compacts). The algorithm used in the code to compute the cross-sectional area of cut, volume of cut, and effective depth of cut for each cutter does not impose any restrictions on the size of the cutters considered. The geometry must simply be that of a circular disk affixed to the bit body at some backrake angle greater than zero (i.e., a negative rake). The algorithms used to compute cutter wearflat temperatures and estimate new wearflat dimensions make use of tabulated data that are accurate only for 0.5 inch and 0.75 inch diameter 
compacts. This, in principle, restricts input cutter radii to one-half those values. Calculations for other input radii are done by interpolating or extrapolating on the tabulated data.

The cutter backrake angle defines the degree to which the circular cutter compact profile assumes an elliptical geometry. This angle thus helps define the cutting profile and the resulting cutter forces. In general, a $20^{\circ}$ backrake seems to be optimal for hard-rock drilling with PDC cutters [8], while $5-15^{\circ}$ has been found to minimize cutter forces in softer rocks [8,37]. The input value for the nominal backrake angle must be between 0 and $90^{\circ}$.

The cutter convective cooling coefficient is a function of the hydraulics design of the bit (location and design of fluid ports), the type of drilling fluid, and the flow rate. Shown in Figures D-1 and D-2 are laboratory data obtained in our earlier work $[23,27]$. Convective cooling coeffcients for water flowing past a single instrumented PDC cutter are shown for a uniform flow field and for a low-pressure ( 80 psi) waterjet flow field. These data are used in Figure D-3 to estimate typical downhole cooling coefficients for a gage cutter on a bit with both air and water cooling. It is seen that coefficients on the order of $1761 \mathrm{Btu} / \mathrm{hr} \mathrm{ft}{ }^{20} \mathrm{~F}\left(1.0 \mathrm{~W} / \mathrm{cm}^{20} \mathrm{C}\right)$ with water-based drilling quds are typical. With air cooling, coefficients on the order of 88 $\mathrm{Btu} / \mathrm{hr} \mathrm{ft}^{20} \mathrm{~F}\left(0.05 \mathrm{~W} / \mathrm{cm}^{20} \mathrm{C}\right)$ are more common. Effects of fluid port placement, fluid properties, and poor cutter cleaning are discussed in our previous work $[23,27]$.

Because cutter compact radii, backrake angles, and cooling coefficients are not likely to vary significantly over the face of any given bit design, these quantities are entered into the program as relative quantities, i.e. the actual value divided by a nominal value. For instance, if all cutters are assumed identical on a given bit, the parameters $R R(J), B R(J)$, and $H R(J)$ would all be 1.0 for each cutter.

The sign of the relative cooling coefficient is used to identify the type of each cutter. Positive values of the relative cooling coefficients denote type A cutters, which are governed by the penetrating force correlation constants entered for that cutter type in the operating conditions data file. Negative values of the relative cooling coefficient denote type $B$ cutters, which are governed by a separate set of correlation constants entered in the operating conditions data file.

The radial, angular, and longitudinal positions of the cutters are by far the most important design parameters considered by the program. These positions define the center of each cutter compact in the $\mathrm{x}^{\prime}, \mathrm{y}^{\prime}, \mathrm{z}^{\prime}$ coordinate system (see Figure 31). They determine the cutting profile of the bit and the distribution of cutting loads among the various cutters. The radial position, $R P$, is the distance in an $x^{\prime}, y^{\prime}$ plane from the longitudinal $\left(z^{\prime}\right)$ axis of the bit to the compact center. Cutters must be entered into the code in ascending 


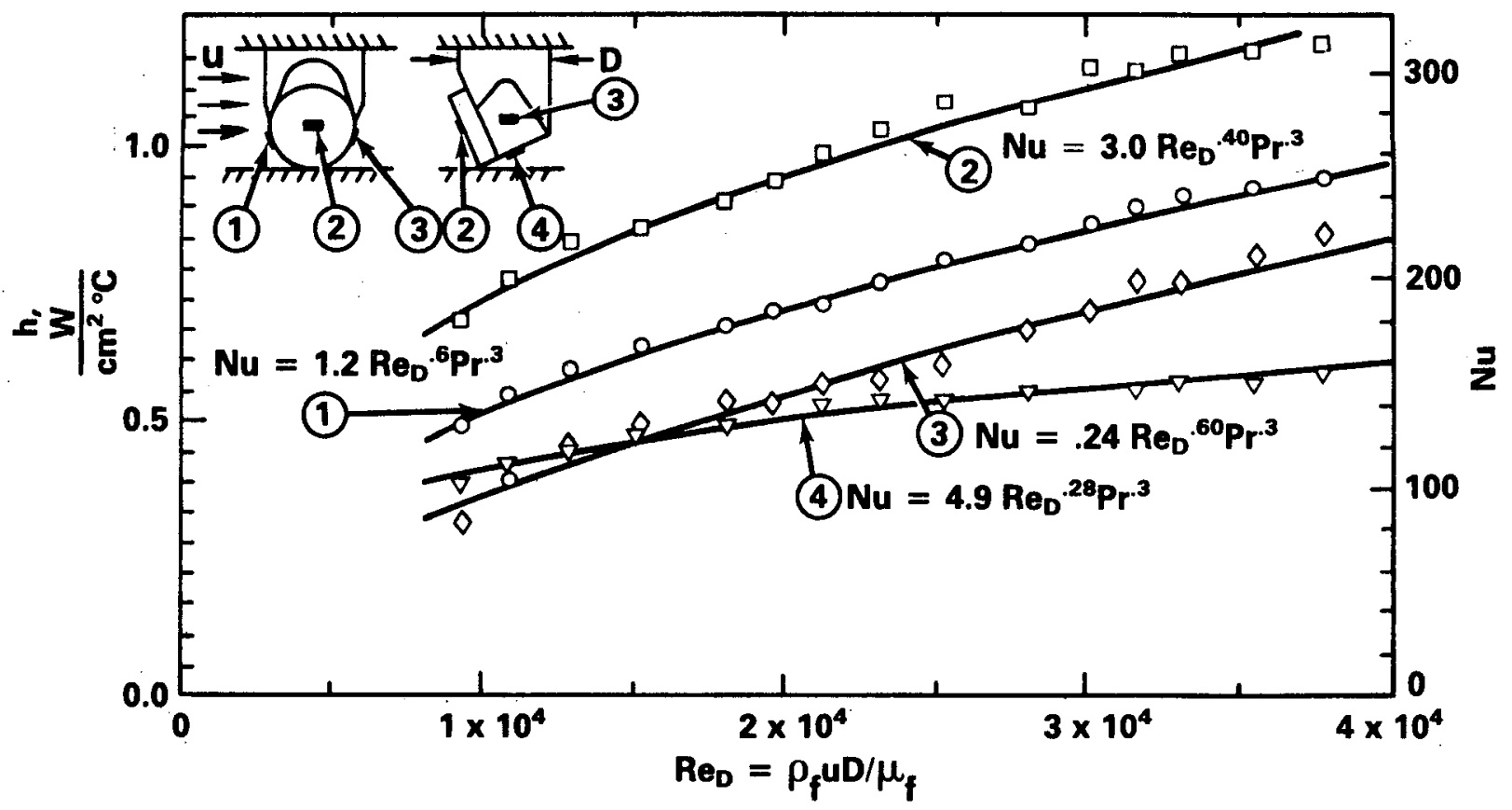

Figure D-1 - Measured heat transfer coefficients for various locations on a stud-mounted 0.5-inch diameter compact cutter due to a uniform flow of water past the cutter (from Ref. 23). 


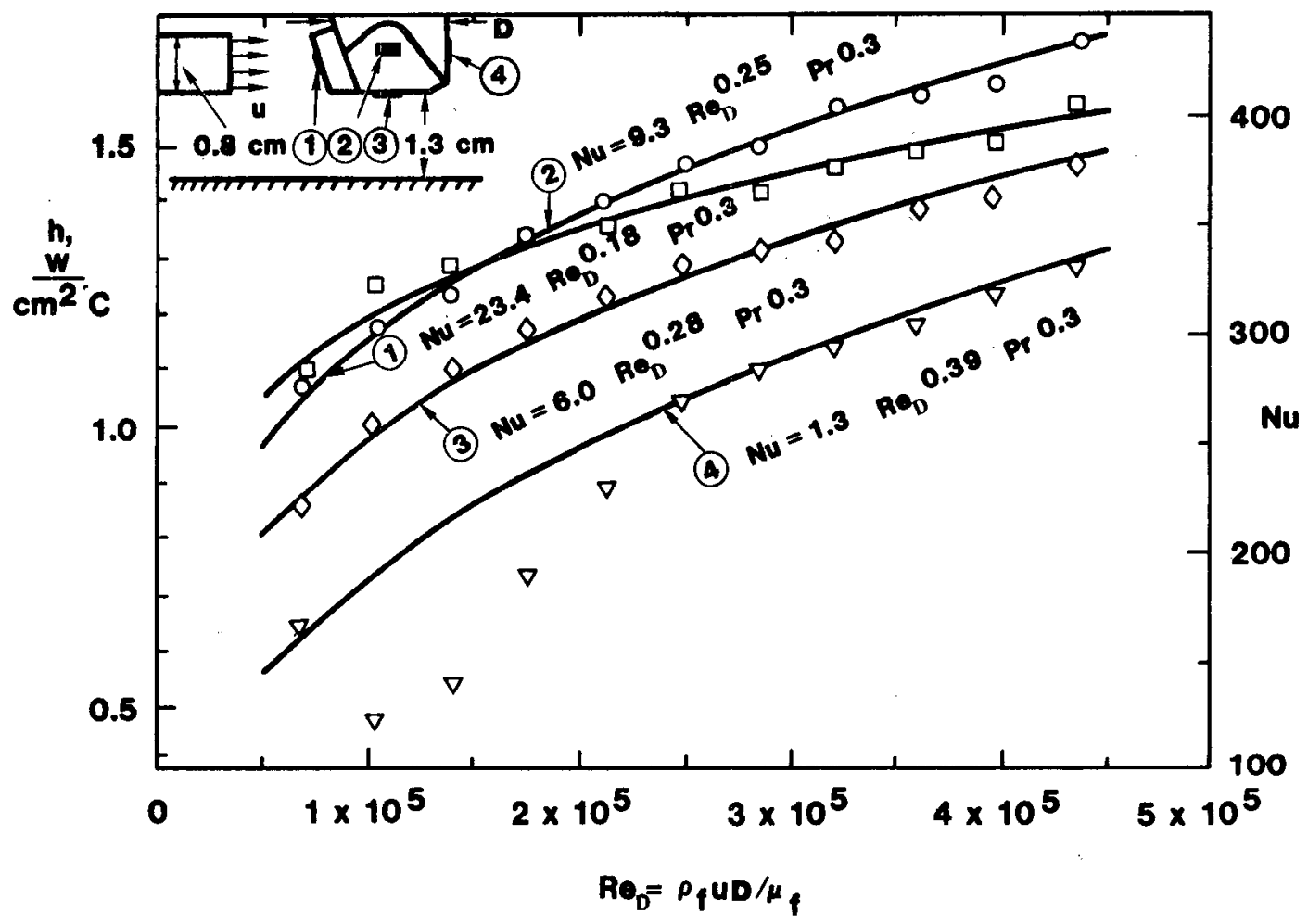

Figure D-2 - Measured heat transfer coefficients for various locations on a stud-mounted 0.5 -inch diameter compact cutter due to impingement of a low-pressure ( 80 psi) waterjet (from Ref. 27). 


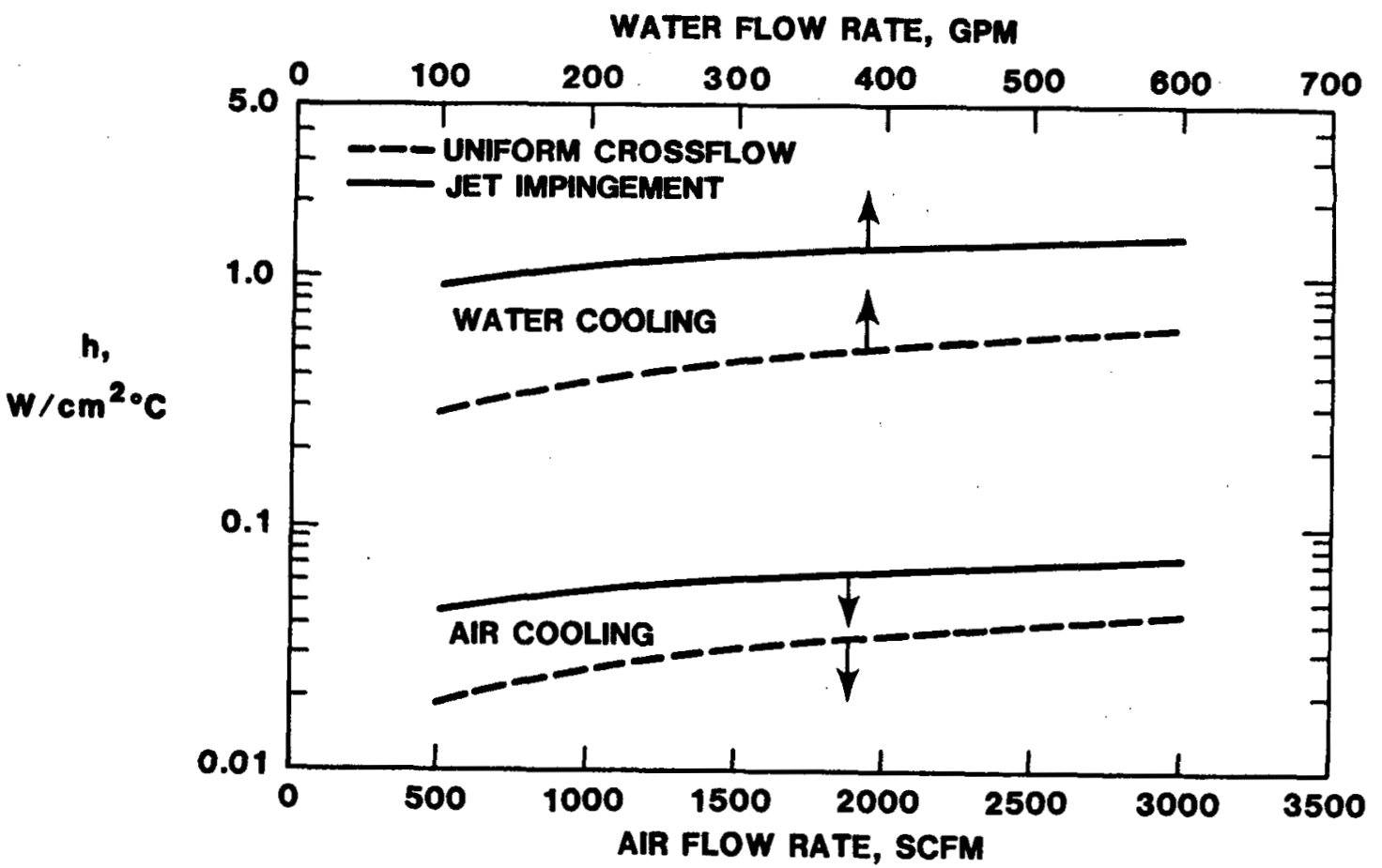

Figure D-3 - Mean PDC cutter heat transfer coefficients under typical air and water drilling conditions (from Ref. 27). 
radial order (i.e., increasing RP), although more than one cutter may share the same radial position. The angular position, AP, of the cutter is also measured in an $x^{\prime}-y^{\prime}$ plane, counterclockwise from the $x^{\prime}$-axis (looking at the face of the bit). The longitudinal position, $\mathrm{H}^{\prime}$, is the distance measured parallel to the $z^{\prime}$-axis from the center of the cutter compact to an arbitrary reference plane parallel to the $x^{\prime}-y^{\prime}$ axes. It is convenient to make the reference plane pass through the center of one of the cutters (i.e., $H^{\prime}=0$ for that cutter). In any case, cutters with positive longitudinal positions are defined as those that lie ahead of the reference plane, in the drilling direction.

The cutter inclination angle, AI, is the angle at which the cutter is mounted on the bit, i.e. the angle between the longitudinal $\left(z^{\prime}\right)$ axis of the bit and the minor axis of the elliptical cutter profile. In a stud-mounted bit, this is equivalent to the angle between the longitudinal axis of the bit and the longitudinal axis of the cutter stud. Most cutters are mounted in holes drilled roughly perpendicular to the surface of the bit body; thus the inclination angles are generally described approximately by the bit body profile. Cutters mounted on convex surfaces on the bit body are defined as having positive inclination angles. Concave surfaces are sometimes designed in the PDC bit bodies in the center of the bit to create a cone of rock that helps stabilize the bit while drilling (see, for example, Figure 30). Cutters mounted in such concave regions would generally have negative inclination angles.

In principle, there is no limitation on the number of cutters considered by PDCWEAR. There is a parameter, MXCUT, that is used to dimension arrays in several subroutines for storing cutter-related quantities. This parameter is currently set at 48 and must be increased in size if a larger number of cutters is to be input to the program. To minimize RAM storage requirements while running the program, parameter MXCUT can be reduced to a value equal to the number of cutters on the bit.

\section{Initial Cutter Wear Configuration File}

This file describes the cutter wearflats that are to be initially considered by PDCWEAR. The following data are read by the program:

$$
\begin{aligned}
& \operatorname{READ}(, 419)(\mathrm{J}, \mathrm{WL}(\mathrm{J}), \mathrm{WW}(\mathrm{J}), \operatorname{AW}(\mathrm{J}), \mathrm{J}=1, \mathrm{NC}) \\
& \text { FORMAT(1X, I3, } 1 \mathrm{X}, \mathrm{F} 10.3,1 \mathrm{X}, \mathrm{F} 10.3,1 \mathrm{X}, \mathrm{F} 10.4)
\end{aligned}
$$

where

$$
\begin{aligned}
& \text { WL }(J) \text { - length of wearflat of } j \text { th cutter, } L \text { (inches), measured in the } \\
& \text { center of the wearflat in the direction parallel to cutter } \\
& \text { travel } \\
& \begin{array}{l}
\text { WW }(J) \text { - width of wearflat of } j \text { th cutter, w (inches), measured at the } \\
\text { diamond face }
\end{array} \\
& \text { AW }(J) \text { - wearflat area of } j \text { th cutter, } A_{W}\left(\text { inch }^{2}\right)
\end{aligned}
$$

These parameters refer to the portion of the wearflat in direct contact with the rock surface being cut.

The algorithms used to compute cutter performance parameters consider the three wear quantities to be independent. The wearflat width affects the cutting profile and thus the cross-sectional area of cut, volume of cut, and 
effective depth of cut. The wearflat area defines the cutter penetrating force that develops at a given depth of cut and the frictional heat generated at the cutter/rock interface. The wearflat length is a variable in the wearflat temperature equation, Eq. 31, which predicts an increase in temperature with wearflat length.

The algorithm used to estimate new cutter wear configurations based on computed wear ratios does require the three wearflat dimensions for each cutter to be consistent. For the hard-rock wear mode, Eqs. 38, 39, and 40 relate the wearflat width and length to the wearflat area. For the soft-rock wear mode, Eqs. 45-47 are more relevant (see subsection 3.4). It is important that initial wear configurations for each cutter conform with the appropriate equations if progressive wear calculations are to be made after the initial performance calculations are complete.

\section{Operating Parameter Data File}

This file defines the intrinsic drillability of the rock, its thermal properties, the rotary speed of the bit, the downhole cooling fluid temperature, and the penetration rates for which performance calculations are desired. The data are read by the program in the following format:

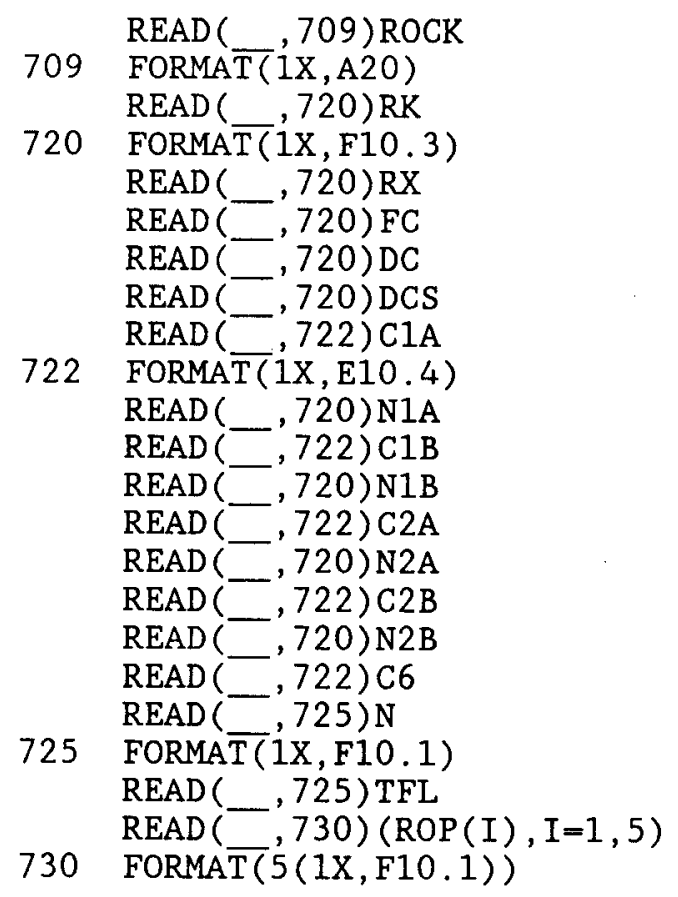

where

ROCK - alphanumeric name for the rock associated with the listed values of the below parameters

$\mathrm{RK}$ - thermal conductivity of the rock, $\mathrm{k}_{2}$ ( $\mathrm{B} f \mathrm{~h} / \mathrm{hr} f t^{\circ} \mathrm{F}$ )

$\mathrm{RX}$ - thermal diffusivity of the rock, $\chi_{2}{ }^{2}$ ( $\mathrm{ft}^{2} / \mathrm{hr}$ )

FC - friction coefficient between the cutter and the rock, $\mu$ 


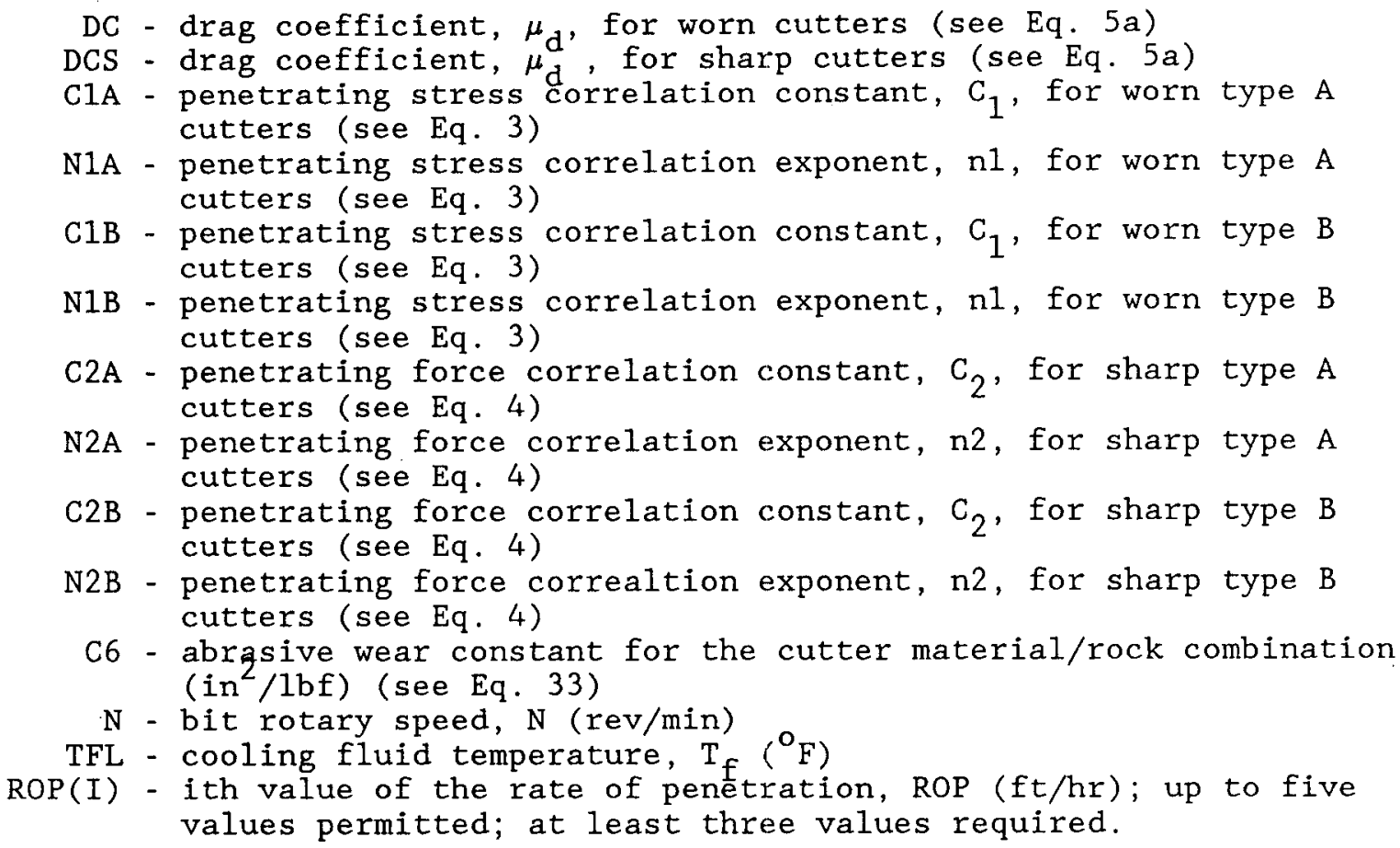

The ROCK parameter is used simply to identify the material associated with the listed parameters. An alphanumeric name up to 20 characters in length may be entered here.

The thermal conductivity, $\mathrm{RK}$, and diffusivity, $\mathrm{RX}$, of the rock affect cutter wearflat temperatures because they control the amount of frictional heat generated at the wearflat that the rock absorbs. Typical values for the conductivity range from $0.35 \mathrm{Btu} / \mathrm{hr} \mathrm{ft}{ }^{\circ} \mathrm{F}$ for certain shales to over $2.9 \mathrm{Btu} / \mathrm{hr}$ $\mathrm{ft}^{\circ} \mathrm{F}$ for anhydrite. Reference [51] contains an extensive compilation of thermal conductivities for most rock types encountered in drilling. The thermal diffusivity is a collection of rock properties:

$$
\chi_{2}=\mathrm{k}_{2} /\left(\rho_{2} \mathrm{c}_{2}\right)
$$

where $\rho_{2}$ is the rock density and $c_{2}$ is the rock specific heat.

The friction coefficient, FC, between the cutter and the rock is one of the most important parameters in the wearflat temperature equation, Eq. 31. This parameter essentially determines what fraction of the cutting power expended by the cutter is converted to frictional heat. Values measured in 
the laboratory [19] with four fluid types, four rock types, and two cutting speeds range from 0.03 to 0.31 and are listed in Table D-1.

TABLE D-1

MEASURED PDC/ROCK FRICTION COEFFICIENTS (REF, 19)

\begin{tabular}{|c|c|c|c|c|c|}
\hline \multirow[b]{2}{*}{ ROCK TYPE } & \multirow{2}{*}{$\begin{array}{l}\text { CUTTING } \\
\text { SPEED } \\
(\mathrm{m} / \mathrm{s}) \\
\end{array}$} & \multicolumn{4}{|c|}{ FRICTION COEFFICIENT } \\
\hline & & Water & Mud & Diesel & Air \\
\hline \multirow[t]{2}{*}{ Marble } & 1 & 0.20 & 0.17 & 0.34 & 0.33 \\
\hline & 5 & 0.15 & 0.11 & 0.13 & 0.15 \\
\hline \multirow[t]{2}{*}{ Sands tone } & 1 & 0.03 & 0.04 & 0.05 & 0.10 \\
\hline & 5 & 0.05 & 0.04 & 0.30 & 0.30 \\
\hline \multirow[t]{2}{*}{ Granite } & 1 & 0.09 & -- & -- & 0.18 \\
\hline & 5 & 0.05 & - & - - & 0.14 \\
\hline \multirow[t]{2}{*}{ Shale } & 1 & 0.09 & - & - - & 0.18 \\
\hline & 5 & 0.06 & -- & - - & 0.15 \\
\hline
\end{tabular}

The cutter drag coefficients for sharp and worn cutters have been shown to be functions of rock type and fluid environment (Figures 13, 14, 15, and 17). The drag coefficient is not strongly dependent on depth of cut, so a mean value is all that the program requires. Since the drag coefficient for sharp cutters may be substantially higher than those for worn cutters over the range of interest in depth of cut, the program requires values, DC and DCS, for each of these classes of cutter wear.

Because of the possibility of using waterjet assistance in bit design, different parameters describing the intrinsic drillability are accepted for two types of cutter, type A and type B. The cutter penetrating stress (for worn cutters) and penetrating force (for sharp cutters) correlation constants and exponents for both types ( $A$ and $B$ ) must be input to the program, although dummy (zero) values may be used for type B cutters if none of the cutters are provided jet assistance. These correlation constants are determined from experimental data, as described previously. In the absence of rock- and environment-specific data for a particular application, the values shown on Figures 10, 11, 12, and 16 may be used for estimating bit performance.

The abrasive wear constant may be determined using single-cutter wear data as described by the equations developed in Ref. 32 . In the absence of rock- and environment-specific data for a parficular application, the value determined for hard sandstones, $\mathrm{C}_{6}=6.89 \times 10^{-13} \mathrm{in}^{2} / \mathrm{lbf}$, may be used for estimating bit performance. Since this parameter is used only to calculate the length of hole drilled, the relative performance of different bit designs can be determined by using any non-zero value of $\mathrm{C}_{6}$. 
The rotary speed of the bit is important because it defines the cutting speeds and thus the frictional heating rate and temperatures of the cutters. Low rotary speeds, 50-200 RPM, are desirable for hard-rock drilling with PDC bits in order to minimize thermal wear effects $[20,31]$. Downhole turbine speeds up to 1000 RPM may be used successfully if the rock formation is very soft [31]. Cutter forces may be reduced by increasing rotary speed while maintaining a constant penetration rate. Generally, however, the higher speeds tend to increase cutter temperatures more than the lower penetrating stresses reduce them, resulting in higher net temperatures. Also, for a given WOB and drilled borehole length, cutters travel farther abrading against the rock surface when the bit is rotated at a higher speed.

The downhole fluid temperature defines the lowest possible wearflat temperature for the analysis. Downhole fluid temperatures generally exceed $125^{\circ} \mathrm{F}$ in petroleum drilling and $200^{\circ} \mathrm{F}$ in geothermal drilling. Computer models have been developed for predicting downhole fluid conditions under a wide variety of conditions [52].

Since knowledge of bit performance over a range of penetration rates is usually desired, the program repeats the calculations at the specified rotary speed for up to five different penetration rates specified by the user. The only requirements on the specified penetration rates is that they be entered in ascending order. Fewer than five penetration rates may be specified by entering zero values in the appropriate data fields after the highest penetration rate specified; however, at least three penetration rates must be specified if cutter wear is to be calculated by the program (the program uses wear ratios computed for the third penetration rate specified). Care should be taken to ensure that the resulting depths of cut do not extend beyond the range for which the cutter stress and force correlation constants and exponents are valid. Practical values for the penetration rate generally range from 5 to $100 \mathrm{ft} / \mathrm{hr}$. 


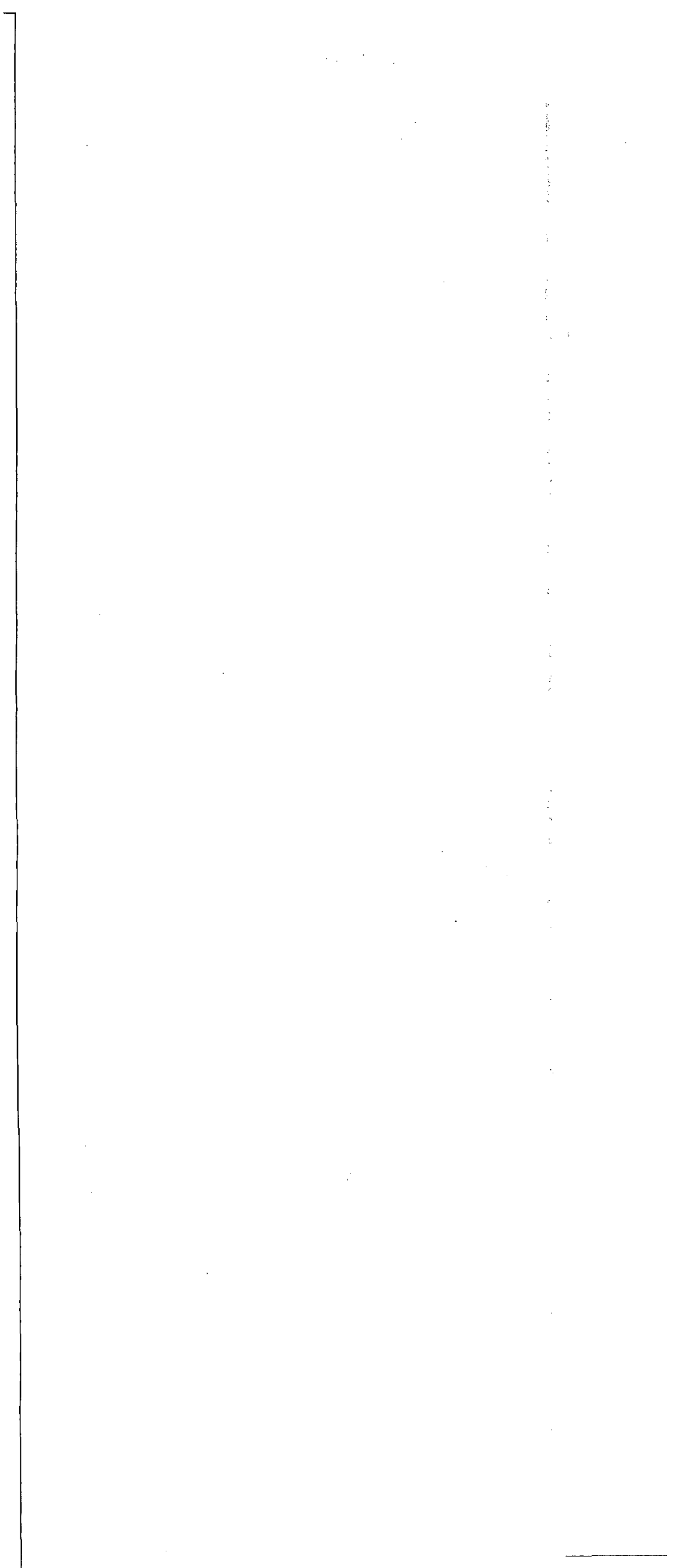


APPENDIX E

LISTING OF FORTRAN PROGRAM FORMAT

$-165$ 
PROGRAM FORMAT

REAL N1A, N1B, N2A, N2B

CHARACTER *11 INNAME, PRNAME, WDNAME, RKNAME

CHARACTER $* 20$ ROCK

DIMENSION RP(50), AP(50), $\operatorname{HPP}(50), \operatorname{AI}(50), \operatorname{RR}(50)$,

$1 \mathrm{BR}(50), \operatorname{HR}(50), \mathrm{WL}(50), \operatorname{WW}(50), \mathrm{AW}(50)$

INNAME $=$;

IDISK $=10$

WDNAME $=$ '

IDISK7 $=7$

RKNAME $=$,

IDISK $8=8$

WRITE $(5,5)$

5 FORMAT (" DO YOU WANT TO CREATE BIT DESIGN DATA FILE $(Y=1)$ ?")

IFLAG $=0$

READ (5,*)IFLAG

IF (IFLAG. NE.1)GO TO 305

10 WRITE $(5,101)$

101 FORMAT $(/$ ' ENTER NAME OF FILE TO CONTAIN BIT DESIGN DATA'

1 ' (11 CHAR. OR LESS, INCLUDING . EXTENSION)')

READ (5,' (A11)') INNAME

IF (INNAME. EQ.' ') GO TO 10

OPEN (IDISK, FILE=INNAME, STATUS= ' NEW' )

WRITE $(5,20)$

20 FORMAT(' ENTER NOMINAL RADIUS OF CUTTER COMPACTS, RNOM' )

$\operatorname{READ}(5, *)$ RNOM

WRITE $(5,22)$

22 FORMAT(' ENTER NOMINAL CUTTER BACKRAKE ANGLE, BNOM')

$\operatorname{READ}(5, *)$ BNOM

WRITE $(5,25)$

25 FORMAT(' ENTER NOMINAL CUTTER CONVECTIVE COOLING COEFFICIENT,

1 ' HNOM')

$\operatorname{READ}(5$, *) HNOM

WRITE (IDISK, 102)RNOM, BNOM, HNOM

102 FORMAT (1X,F10.3, 1X, F10.2, 1X, F10.2)

WRITE $(5,30)$

30 FORMAT(' ENTER NUMBER OF CUTTERS, NC')

$\operatorname{READ}(5, *) \mathrm{NC}$

DO $11 \mathrm{~J}=1, \mathrm{NC}$

WRITE $(5,35) \mathrm{J}$

35 FORMAT(' ENTER RADIAL POSITION, RP, FOR CUTTER ', I3)

$\operatorname{READ}(5, *) \operatorname{RP}(\mathrm{J})$

11 CONTINUE

DO $12 \mathrm{~J}=1, \mathrm{NC}$

WRITE $(5,36) \mathrm{J}$

36 FORMAT (' ENTER ANGULAR POSITION, AP, FOR CUTTER ', I3)

$\operatorname{READ}(5$, *) AP (J)

12 CONTINUE

DO $13 \mathrm{~J}=1$, NC

WRITE ( 5, 37) J

37 FORMAT (' ENTER LONGITUDINAL POSITION, HPP, FOR CUTTER ', I3)

$\operatorname{READ}(5$, *) $\operatorname{HPP}(\mathrm{J})$

13 CONTINUE

DO $14 \mathrm{~J}=1$, NC

WRITE $(5,38) \mathrm{J}$

38 FORMAT(' ENTER INCLINATION ANGLE, AI, FOR CUTTER ',I3)

$\operatorname{READ}(5, *) A I(J)$

14 CONTINUE

DO $15 \mathrm{~J}=1, \mathrm{NC}$

WRITE $(5,39) \mathrm{J}$

39 FORMAT(' ENTER RELATIVE COMPACT RADIUS, RR, FOR CUTTER ', I3)

$\operatorname{READ}(5, *) \operatorname{RR}(\mathrm{J})$

15 CONTINUE

DO $16 \mathrm{~J}=1$, NC

WRITE $(5,40) \mathrm{J}$

40 FORMAT(' ENTER RELATIVE BACKRAKE ANGLE, BR, FOR CUTTER ',I3)

$\operatorname{READ}(5, *) \operatorname{BR}(J)$

16 CONTINUE

DO $17 \mathrm{~J}=1$, NC

WRTTE $(5,41) \mathrm{J}$

41 FORMAT(' ENTER RELATIVE CONVECTIVE HEAT TRANSFER COEFFICIENT,'

1 ' $\mathrm{HR}$, FOR CUTTER ' I3/' (NEGATIVE IF'

1 ' TYPE B CUTTER)')

$\operatorname{READ}(5, *) \operatorname{HR}(\mathrm{J})$

17 CONTINUE

DO $18, J=1$, NC

WRITE (IDISK, 119) J $, R P(J), \operatorname{AP}(J), \operatorname{HPP}(J), A I(J)$,

$1 \operatorname{RR}(J), \operatorname{BR}(J), \operatorname{HR}(J)$

119 FORMAT ( $1 \mathrm{X}$, I $3,1 \mathrm{X}, \mathrm{F} 7,3,1 \mathrm{X}, \mathrm{F} 7.3,1 \mathrm{X}, \mathrm{F} 7.3$

1 IX,F7.3, 1X,F7. 3, 1X,F7.3, 1X,F7, 3)

18 CONTINUE

CLOSE (IDISK, STATUS $=$ 'KEEP')

305 WRITE $(5,306)$ 


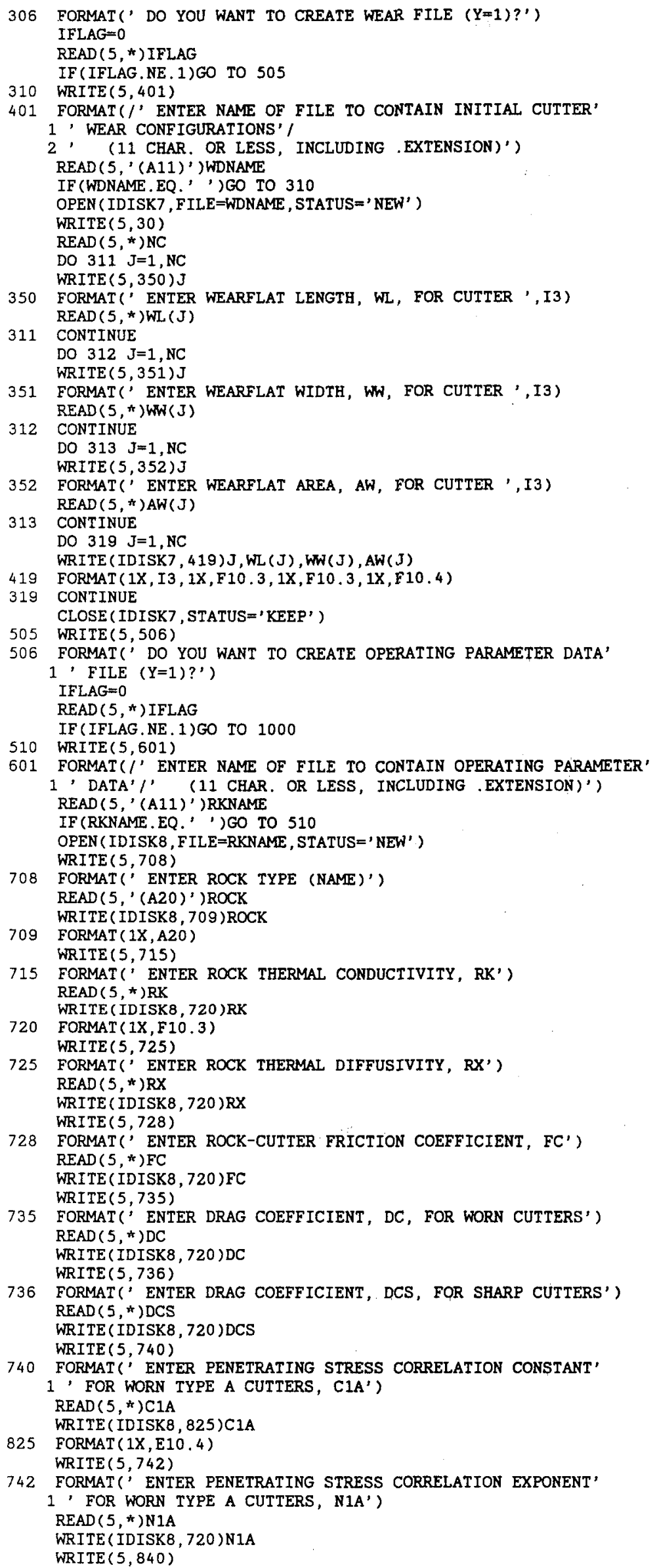


840 FORMAT(' ENTER PENETRATING STRESS CORRELATION CONSTANT'

1 ' FOR WORN TYPE B CUTTERS, C1B')

$\operatorname{READ}(5, \pi) \mathrm{C} 1 \mathrm{~A}$

WRITE(IDISK8, 825)C1A

WRITE $(5,842)$

842 FORMAT (' ENTER PENETRATING STRESS CORRELATION EXPONENT'

1 ' FOR WORN TYPE B CUTTERS, N1B')

$\operatorname{READ}(5, *) \mathrm{N} 1 \mathrm{~B}$

WRITE(IDISK8, 720)N1B

WRITE $(5,746)$

746 FORMAT (' ENTER PENETRATING FORCE CORRELATION CONSTANT'

1 ' FOR SHARP TYPE A CUTTERS, C2A')

$\operatorname{READ}(5, *) C 2 A$

WRITE (IDISK8, 825)C2A

WRITE $(5,747)$

747 FORMAT'; ENTER PENETRATING FORCE CORRELATION EXPONENT'

1 ' FOR SHARP TYPE A CUTTERS, N2A')

$\operatorname{READ}(5, *) \mathrm{N} 2 \mathrm{~A}$

WRITE (IDISK8, 720)N2A

WRITE $(5,846)$

846 FORMAT(' ENTER PENETRATING FORCE CORRELATION CONSTANT'

1 ' FOR SHARP TYPE B CUTTERS, $\left.\mathrm{C}^{\prime} \mathrm{B}^{\prime}\right)$

$\operatorname{READ}(5, *) \mathrm{C} 2 \mathrm{~A}$

WRITE(IDISK8, 825)C2A

WRITE $(5,847)$

847 FORMAT(' ENTER PENETRATING FORCE CORRELATION EXPONENT'

1 ' FOR SHARP TYPE B CUTTERS, N2B')

$\operatorname{READ}(5, *) \mathrm{N} 2 \mathrm{~B}$

WRITE (IDISK8, 720)N2B

WRITE $(5,754)$

754 FORMAT (' ENTER ABRASIVE WEAR CONSTANT, C6')

$\operatorname{READ}(5, *) \mathrm{C} 6$

WRITE (IDISK8, 825)C6

WRITE $(5,755)$

755 FORMAT(' ENTER BIT ROTARY SPEED, N')

$\operatorname{READ}(5, *) \operatorname{RPM}$

WRITE (IDISK8, 790)RPM

790 FORMAT (1X, F10.1)

WRITE $(5,760)$

760 FORMAT(' ENTER DOWNHOLE COOLING FLUID TEMPERATURE, TFL')

$\operatorname{READ}(5, *)$ TFL

WRITE (IDISK8, 790) TFL

WRITE $(5,765)$

765 FORMAT(' ENTER BIT PENETRATION RATES, ROP(1), ROP(2), ROP(3),'

1 ' $\operatorname{ROP}(4)$, AND ROP (5)')

READ ( 5, *) ROP1, ROP2, ROP3, ROP4, ROP 5

WRITE (IDISK8, 730) (ROP1, ROP2 , ROP3, ROP4, ROP5)

730 FORMAT (1X,F10.1, 1X,F10.1, 1X,F10.1, 1X, F10.1, 1X,F10.1)

CLOSE ( IDISK8, STATUS=' KEEP')

1000 CONTINUE

END 
APPENDIX F

LISTING OF PDCWEAR INPUT FILES USED

IN DEMONSTRATION ANALYSIS 
TABLE $F-1$

PDCWEAR INPUT FILE CONTAINING BIT DESIGN DATA FOR DEMONSTRATION ANALYSIS (BITDES.DAT)

$\begin{array}{lll}0.375 & 20.00 & 1700.00\end{array}$

$\begin{array}{rrrrrrrr}1 & 0.350 & 0.000 & 0.625 & -15.000 & 1.000 & 1.000 & 1.000 \\ 2 & 0.800 & 215.000 & 0.760 & -10.000 & 1.000 & 1.000 & 1.000 \\ 3 & 1.250 & 110.000 & 0.895 & -5.000 & 1.000 & 1.000 & 1.000 \\ 4 & 1.600 & 45.000 & 1.000 & -2.000 & 1.000 & 1.000 & 1.000 \\ 5 & 1.950 & 285.000 & 1.000 & 0.000 & 1.000 & 1.000 & 1.000 \\ 6 & 2.300 & 165.000 & 1.000 & 2.000 & 1.000 & 1.000 & 1.000 \\ 7 & 2.495 & 0.000 & 0.990 & 5.000 & 1.000 & 1.000 & 1.000 \\ 8 & 2.635 & 240.000 & 0.970 & 10.000 & 1.000 & 1.000 & 1.000 \\ 9 & 2.775 & 120.000 & 0.940 & 15.000 & 1.000 & 1.000 & 1.000 \\ 10 & 2.915 & 320.000 & 0.898 & 20.000 & 1.000 & 1.000 & 1.000 \\ 11 & 3.055 & 200.000 & 0.844 & 25.000 & 1.000 & 1.000 & 1.000 \\ 12 & 3.195 & 80.000 & 0.776 & 30.000 & 1.000 & 1.000 & 1.000 \\ 13 & 3.335 & 290.000 & 0.694 & 35.000 & 1.000 & 1.000 & 1.000 \\ 14 & 3.475 & 170.000 & 0.594 & 40.000 & 1.000 & 1.000 & 1.000 \\ 15 & 3.615 & 50.000 & 0.473 & 45.000 & 1.000 & 1.000 & 1.000 \\ 16 & 3.735 & 20.000 & 0.347 & 47.500 & 1.000 & 1.000 & 1.000 \\ 17 & 3.835 & 260.000 & 0.222 & 50.000 & 1.000 & 1.000 & 1.000 \\ 18 & 3.915 & 140.000 & 0.104 & 52.500 & 1.000 & 1.000 & 1.000 \\ 19 & 3.975 & 335.000 & 0.000 & 55.000 & 1.000 & 1.000 & 1.000 \\ 20 & 3.975 & 215.000 & 0.000 & 55.000 & 1.000 & 1.000 & 1.000 \\ 21 & 3.975 & 95.000 & 0.000 & 55.000 & 1.000 & 1.000 & 1.000\end{array}$


TABLE $F-2$

PDCWEAR INPUT FILE CONTAINING INITIAL WEAR CONFIGURATION DATA FOR DEMONSTRATION ANALYSIS (WEARCF.DAT)

$\begin{array}{rlll}1 & 0.000 & 0.000 & 0.0000 \\ 2 & 0.000 & 0.000 & 0.0000 \\ 3 & 0.000 & 0.000 & 0.0000 \\ 4 & 0.000 & 0.000 & 0.0000 \\ 5 & 0.000 & 0.000 & 0.0000 \\ 6 & 0.000 & 0.000 & 0.0000 \\ 7 & 0.000 & 0.000 & 0.0000 \\ 8 & 0.000 & 0.000 & 0.0000 \\ 9 & 0.000 & 0.000 & 0.0000 \\ 10 & 0.000 & 0.000 & 0.0000 \\ 11 & 0.000 & 0.000 & 0.0000 \\ 12 & 0.000 & 0.000 & 0.0000 \\ 13 & 0.000 & 0.000 & 0.0000 \\ 14 & 0.000 & 0.000 & 0.0000 \\ 15 & 0.000 & 0.000 & 0.0000 \\ 16 & 0.000 & 0.000 & 0.0000 \\ 17 & 0.000 & 0.000 & 0.0000 \\ 18 & 0.000 & 0.000 & 0.0000 \\ 19 & 0.000 & 0.000 & 0.0000 \\ 20 & 0.000 & 0.000 & 0.0000 \\ 21 & 0.000 & 0.000 & 0.0000\end{array}$


TABLE $F-3$

PDCWEAR INPUT FILE CONTAINING OPERATING CONDITIONS

FOR DEMONSTRATION ANALYSIS (OPCOND.DAT)

\section{SIERRA WHITE GRANITE}

1.300

0.033

0.070

0.550

0.750

$0.1340 \mathrm{E}+06$

0.420

$0.3260 \mathrm{E}+06$

0.930

$0.2550 \mathrm{E}+05$

1.640

$0.2550 \mathrm{E}+05$

1.640

$0.6890 \mathrm{E}-12$

100.0

80.0

10.0

20.0

30.0

40.0

50.0 
APPENDIX G

PARTIAL PDCWEAR OUTPUT LISTING

FOR DEMONSTRATION ANALYSIS 
1

*** INPUT DATA *** 21-MAY-87 10:17:37

BIT DESIGN DATA FROM FILE BITDES.DAT :

THERE ARE 21 CUTTERS WITH NOMINAL RADIUS $=0.375 \mathrm{IN}$,

NOMINAL BACKRAKE ANGLE $=20.00 \mathrm{DEG}$.

AND NOMINAL CONVECTIVE COOLING COEFF. $=1700.00 \mathrm{BTU} / \mathrm{HR}-\mathrm{FT} * * 2-F$.

\begin{tabular}{|c|c|c|c|c|c|c|c|}
\hline & $\begin{array}{l}\text { RADIAL } \\
\text { POS. } \\
\text { (IN.) }\end{array}$ & $\begin{array}{l}\text { ANG. } \\
\text { POS. } \\
\text { (DEG. ) }\end{array}$ & $\begin{array}{l}\text { LONG. } \\
\text { POS. } \\
\text { (IN.) }\end{array}$ & $\begin{array}{l}\text { INCL. } \\
\text { ANGLE } \\
\text { (DEG. ) }\end{array}$ & $\begin{array}{l}\text { REL. } \\
\text { RADIUS }\end{array}$ & $\begin{array}{l}\text { REL . } \\
\text { BACKRAKE } \\
\text { ANGLE }\end{array}$ & $\begin{array}{c}\text { REL . CONV. } \\
\text { COOLING } \\
\text { COEFF . }\end{array}$ \\
\hline 1 & 0.350 & 0.000 & 0.625 & -15.000 & 1.000 & 1.000 & 1.000 \\
\hline 2 & 0.800 & 215.000 & 0.760 & -10.000 & 1.000 & 1.000 & 1.000 \\
\hline 3 & 1.250 & 110.000 & 0.895 & -5.000 & 1.000 & 1.000 & 1.000 \\
\hline 4 & 1. 600 & 45.000 & 1.000 & -2.000 & 1.000 & 1.000 & 1.000 \\
\hline 5 & 1.950 & 285.000 & 1.000 & 0.000 & 1.000 & 1.000 & 1.000 \\
\hline 6 & 2.300 & 165.000 & 1.000 & 2.000 & 1.000 & 1.000 & 1.000 \\
\hline 7 & 2.495 & 0.000 & 0.990 & 5.000 & 1.000 & 1.000 & 1.000 \\
\hline 8 & 2.635 & 240.000 & 0.970 & 10.000 & 1.000 & 1.000 & 1.000 \\
\hline 9 & 2.775 & 120.000 & 0.940 & 15.000 & 1.000 & 1.000 & 1.000 \\
\hline 10 & 2.915 & 320.000 & 0.898 & 20.000 & 1.000 & 1.000 & 1.000 \\
\hline 11 & 3.055 & 200.000 & 0.844 & 25.000 & 1.000 & 1.000 & 1.000 \\
\hline 12 & 3.195 & 80.000 & 0.776 & 30.000 & 1.000 & 1.000 & 1.000 \\
\hline 13 & 3.335 & 290.000 & 0.694 & 35.000 & 1.000 & 1.000 & 1.000 \\
\hline 14 & 3.475 & 170.000 & 0.594 & 40.000 & 1.000 & 1.000 & 1.000 \\
\hline 15 & 3. 615 & 50.000 & 0.473 & 45.000 & 1.000 & 1.000 & 1.000 \\
\hline 16 & 3.735 & 20.000 & 0.347 & 47.500 & 1.000 & 1.000 & 1.000 \\
\hline 17 & 3.835 & 260.000 & 0.222 & 50.000 & 1.000 & 1.000 & 1.000 \\
\hline 18 & 3.915 & 140.000 & 0.104 & 52.500 & 1.000 & 1.000 & 1.000 \\
\hline 19 & 3.975 & 335.000 & 0.000 & 55.000 & 1.000 & 1.000 & 1.000 \\
\hline 20 & 3.975 & 215.000 & 0.000 & 55.000 & 1.000 & 1.000 & 1.000 \\
\hline 21 & 3.975 & 95.000 & 0.000 & 55.000 & 1.000 & 1.000 & 1.000 \\
\hline
\end{tabular}

* NEgative value of RELATIVe COOLING COEFFICIENT

DENOTES TYPE B CUTTER. ALL OTHER CUTTERS ARE TYPE A.

INITIAL WEAR CONFIGURATION DATA FROM FILE WEARCF.DAT :

$\begin{array}{cccc}\text { CUTTER } & \begin{array}{c}\text { WEARFLAT } \\ \text { LENGTH } \\ \text { (IN.) }\end{array} & \begin{array}{c}\text { WEARFLAT } \\ \text { WIDTH } \\ \text { (IN.) }\end{array} & \begin{array}{c}\text { WEARFLAT } \\ \text { AREA } \\ (\text { IN* } 2)\end{array} \\ 1 & 0.000 & 0.000 & 0.0000 \\ 2 & 0.000 & 0.000 & 0.0000 \\ 3 & 0.000 & 0.000 & 0.0000 \\ 4 & 0.000 & 0.000 & 0.0000 \\ 5 & 0.000 & 0.000 & 0.0000 \\ 6 & 0.000 & 0.000 & 0.0000 \\ 7 & 0.000 & 0.000 & 0.0000 \\ 8 & 0.000 & 0.000 & 0.0000 \\ 9 & 0.000 & 0.000 & 0.0000 \\ 10 & 0.000 & 0.000 & 0.0000 \\ 11 & 0.000 & 0.000 & 0.0000 \\ 12 & 0.000 & 0.000 & 0.0000 \\ 13 & 0.000 & 0.000 & 0.0000 \\ 14 & 0.000 & 0.000 & 0.0000 \\ 15 & 0.000 & 0.000 & 0.0000 \\ 16 & 0.000 & 0.000 & 0.0000 \\ 17 & 0.000 & 0.000 & 0.0000 \\ 18 & 0.000 & 0.000 & 0.0000 \\ 19 & 0.000 & 0.000 & 0.0000 \\ 20 & 0.000 & 0.000 & 0.0000 \\ 21 & 0.000 & 0.000 & 0.0000\end{array}$




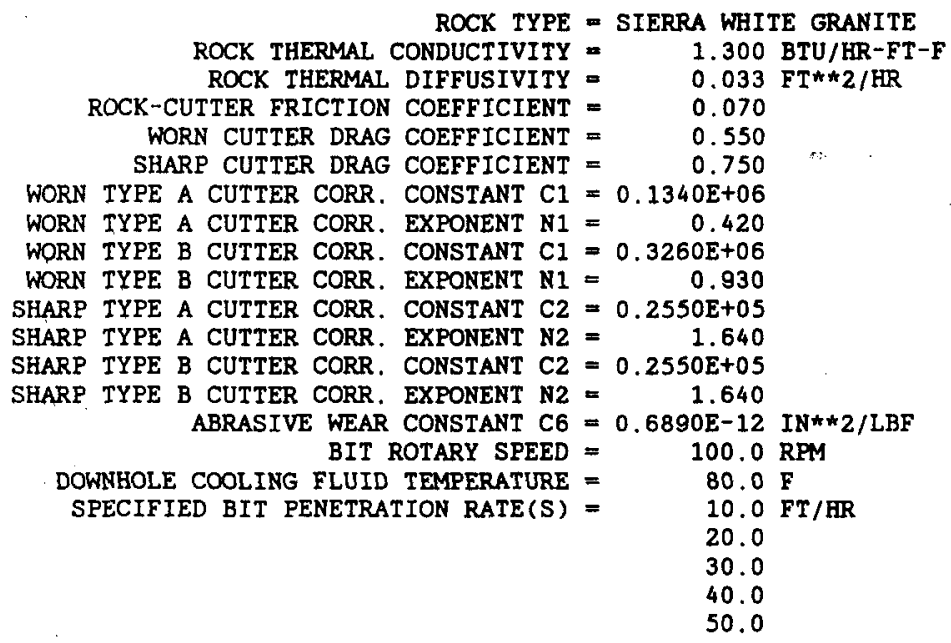




\begin{tabular}{|c|c|c|c|c|c|c|c|c|c|}
\hline NRA & $Y=100$ & & & & & $\therefore \therefore$ & $\Leftrightarrow$ & . & \\
\hline \multicolumn{2}{|c|}{ GEOMETRY-DEPENDEI } & T RESULTS & FOR PENETRA & TION RATE & $=10.00$ & $\mathrm{FT} / \mathrm{HR}$ & & . & \\
\hline CUTTER & $\begin{array}{l}\text { CUTTING } \\
\text { ORDER }\end{array}$ & $\begin{array}{r}\text { CUTTING } \\
\text { HEIGHT } \\
(\text { IN.) }\end{array}$ & $\begin{array}{l}\text { WEARFLAT } \\
\text { LOCATION } \\
\text { XBC } \\
\text { (IN.) }\end{array}$ & $\begin{array}{c}\text { WEARFLAT } \\
\text { LOCATION } \\
\text { ZBC } \\
\text { (IN.) }\end{array}$ & $\begin{array}{l}\text { CUTTER } \\
\text { INCL. } \\
\text { ANGLE } \\
\text { (DEG.) }\end{array}$ & $\begin{array}{l}\text { CUTTER } \\
\text { WEAR } \\
\text { ANGLE } \\
\text { (DEG.) }\end{array}$ & $\begin{array}{l}\text { VOLUME } \\
\text { OF } \\
\text { CUT } \\
\text { (IN**3/ } \\
\text { REV ) }\end{array}$ & $\begin{array}{l}\text { AREA } \\
\text { OF } \\
\text { CUT } \\
\left(I N^{\star \star} 2\right)\end{array}$ & $\begin{array}{l}\text { EFFECTIVE } \\
\text { DEPTH } \\
\text { OF CUT } \\
\text { (IN : ) }\end{array}$ \\
\hline 1 & 20 & 0.645 & 0.205 & 0.967 & -15.000 & -23.149 & 0.0153 & 0.0099 & 0.0521 \\
\hline 2 & 8 & 0.768 & 0.694 & 1.104 & -10.000 & -16.660 & 0.0405 & 0.0090 & 0.0447 \\
\hline 3 & 14 & 0.909 & 1.116 & 1.237 & -5.000 & -20.358 & 0.0558 & 0.0078 & 0.0390 \\
\hline 4 & 18 & 1.018 & 1.544 & 1.366 & -2.000 & -8.296 & 0.0873 & 0.0089 & 0.0437 \\
\hline 5 & 4 & 1.004 & 1.954 & 1.357 & 0.000 & 0.609 & 0.0856 & 0.0070 & 0.0336 \\
\hline 6 & 11 & 1.011 & 2.276 & 1.363 & 2.000 & -3.241 & 0.0823 & 0.0058 & 0.0290 \\
\hline 7 & 21 & 1.010 & 2.525 & 1. 361 & 5.000 & 4.965 & 0.0658 & 0.0042 & 0.0232 \\
\hline 8 & 6 & 0.977 & 2.707 & 1.322 & 10.000 & 11.550 & 0.0551 & 0.0032 & 0.0198 \\
\hline 9 & 13 & 0.953 & 2.869 & 1.293 & 15.000 & 15.392 & 0.0578 & 0.0032 & 0.0197 \\
\hline 10 & 2 & 0.900 & 3.036 & 1. 231 & 20.000 & 20.014 & 0.0645 & 0.0034 & 0.0203 \\
\hline 11 & 9 & 0.853 & 3.204 & 1.172 & 25.000 & 24.940 & 0.0665 & 0.0033 & 0.0200 \\
\hline 12 & 16 & 0.792 & 3.366 & 1.100 & 30.000 & 29.108 & 0.0672 & 0.0032 & 0.0195 \\
\hline 13 & 3 & 0.698 & 3.531 & 0.990 & 35.000 & 34.019 & 0.0742 & 0.0034 & 0.0201 \\
\hline 14 & 10 & 0.605 & 3.700 & 0.876 & 40.000 & 39.773 & 0.0757 & 0.0033 & 0.0197 \\
\hline 15 & 17 & 0.490 & 3.866 & 0.738 & 45.000 & 45.281 & 0.0775 & 0.0032 & 0.0195 \\
\hline 16 & 19 & 0.366 & 4.005 & 0.592 & 47.500 & 49.702 & 0.0637 & 0.0025 & 0.0167 \\
\hline 17 & 5 & 0.228 & 4.123 & 0.431 & 50.000 & 54.162 & 0.0559 & 0.0022 & 0.0150 \\
\hline 18 & 12 & 0.116 & 4.213 & 0.305 & 52.500 & 57.064 & 0.0412 & 0.0016 & 0.0122 \\
\hline 19 & 1 & 0.001 & 4.304 & 0.131 & 55.000 & 67.023 & 0.0162 & 0.0006 & 0.0087 \\
\hline 20 & 7 & 0.008 & 4.302 & 0.143 & 55.000 & 66.192 & 0.0174 & 0.0006 & 0.0093 \\
\hline 21 & 15 & 0.015 & 4. 306 & 0.138 & 55.000 & 67.883 & 0.0152 & 0.0006 & 0.0081 \\
\hline
\end{tabular}

ROCK-DEPENDENT RESULTS FOR PENETRATION RATE $=10.00 \mathrm{FT} /$ HR

\begin{tabular}{|c|c|c|c|c|c|c|c|c|c|}
\hline CUTTER & $\begin{array}{c}\text { VOLUME } \\
\text { OF CUT } \\
\left(\text { IN }^{*}+3 / \mathrm{REV}\right)\end{array}$ & $\begin{array}{l}\text { AREA } \\
\text { OF CUT } \\
(I N * * 2)\end{array}$ & $\begin{array}{l}\text { EFFECTIVE } \\
\text { DEPTH OF CUT } \\
\text { (IN.) }\end{array}$ & $\begin{array}{c}\text { PENETRATING } \\
\text { FORCE } \\
\text { (LBF) }\end{array}$ & $\begin{array}{l}\text { DRAG } \\
\text { FORCE } \\
\text { (LBF) }\end{array}$ & $\begin{array}{l}\text { VERTICAL } \\
\text { FORCE } \\
\text { (LBE) }\end{array}$ & $\begin{array}{l}\text { RADIAL } \\
\text { FORCE } \\
\text { (LBF) }\end{array}$ & $\begin{array}{l}\text { WEARFLAT } \\
\text { TEMPERATURE } \\
\text { (DEG. C) }\end{array}$ & $\begin{array}{l}\text { WEAR } \\
\text { RATIO }\end{array}$ \\
\hline 1 & 0.0153 & 0.0099 & 0.0521 & 200.6 & 150.5 & -184.5 & 78.9 & 26.7 & 1.00 \\
\hline 2 & 0.0405 & 0.0090 & 0.0447 & 155.8 & 116.9 & -149.3 & 44.7 & 26.7 & 2.62 \\
\hline 3 & 0.0558 & 0.0078 & 0.0390 & 124.5 & 93.4 & -116.7 & 43.3 & 26.7 & 3.37 \\
\hline 4 & 0.0873 & 0.0089 & 0.0437 & 150.3 & 112.8 & -148.8 & 21.7 & 26.7 & 5.64 \\
\hline 5 & 0.0856 & 0.0070 & 0.0336 & 97.8 & 73.3 & -97.8 & -1.0 & 26.7 & 4.64 \\
\hline 6 & 0.0823 & 0.0058 & 0.0290 & 76.9 & 57.6 & -76.7 & 4.3 & 26.7 & 4.25 \\
\hline 7 & 0.0658 & 0.0042 & 0.0232 & 53.2 & 39.9 & -53.0 & -4.6 & 26.7 & 3.26 \\
\hline 8 & 0.0551 & 0.0032 & 0.0198 & 41.1 & 30.8 & -40.3 & -8.2 & 26.7 & 2.70 \\
\hline 9 & 0.0578 & 0.0032 & 0.0197 & 40.8 & 30.6 & -39.4 & -10.8 & 26.7 & 2.84 \\
\hline 10 & 0.0645 & 0.0034 & 0.0203 & 42.7 & 32.1 & -40.2 & -14.6 & 26.7 & 3.15 \\
\hline 11 & 0.0665 & 0.0033 & 0.0200 & 41.8 & 31.3 & -37.9 & -17.6 & 26.7 & 3.25 \\
\hline 12 & 0.0672 & 0.0032 & 0.0195 & 39.9 & 29.9 & -34.9 & -19.4 & 26.7 & 3.26 \\
\hline 13 & 0.0742 & 0.0034 & 0.0201 & 41.9 & 31.4 & -34.7 & -23.4 & 26.7 & 3.59 \\
\hline 14 & 0.0757 & 0.0033 & 0.0197 & 40.7 & 30.5 & -31.3 & -26.0 & 26.7 & 3.66 \\
\hline 15 & 0.0775 & 0.0032 & 0.0195 & 39.9 & 29.9 & -28.1 & -28.4 & 26.7 & 3.75 \\
\hline 16 & 0.0637 & 0.0025 & 0.0167 & 30.9 & 23.2 & -20.0 & -23.6 & 26.7 & 3.00 \\
\hline 17 & 0.0559 & 0.0022 & 0.0150 & 25.9 & 19.4 & -15.2 & -21.0 & 26.7 & 2.59 \\
\hline 18 & 0.0412 & 0.0016 & 0.0122 & 18.5 & 13.9 & -10.1 & -15.5 & 26.7 & 1.89 \\
\hline 19 & 0.0162 & 0.0006 & 0.0087 & 10.6 & 8.0 & -4.1 & -9.8 & 26.7 & 1.11 \\
\hline 20 & 0.0174 & 0.0006 & 0.0093 & 11.9 & 8.9 & -4.8 & -10.9 & 26.7 & 1.24 \\
\hline 21 & 0.0152 & 0.0006 & 0.0081 & 9.5 & 7.1 & -3.6 & -8.8 & 26.7 & 0.99 \\
\hline
\end{tabular}

INTEGRATED FORCES AND MOMENTS FOR THE FULL BIT:

SIERRA WHITE GRANITE AT ROP =

10.0

$\begin{array}{llr}\text { TOTAL WOB (LBF) } & =1171.1 \\ \text { DRILLING TORQ. (FT-LBF) } & = & 159.1 \\ \text { X SIDE FORCE (LBF) } & = & 1.4 \\ \text { Y SIDE FORCE (LBF) } & -109.0 \\ \text { RESULTANT SIDE FORCE (LBF) } & =109.0 \\ \text { B. M. ABOUT X-AXIS (FT-LBF) } & 15.3 \\ \text { B. M. ABOUT Y-AXIS (FT-LBF) } & -1.2\end{array}$


GEOMETRY-DEPENDENT RESULTS FOR PENETRATION RATE $=20.00 \mathrm{FT} / \mathrm{HR}$

\begin{tabular}{|c|c|c|c|c|c|c|c|c|c|}
\hline CUTTER & $\begin{array}{l}\text { CUTTING } \\
\text { ORDER }\end{array}$ & $\begin{array}{r}\text { CUTTING } \\
\text { HEIGHT } \\
\text { (IN.) }\end{array}$ & $\begin{array}{l}\text { WEARFLAT } \\
\text { LOCATION } \\
\text { XBC } \\
\text { (IN . ) }\end{array}$ & $\begin{array}{l}\text { WEARFLAT } \\
\text { LOCATION } \\
\text { ZBC } \\
\text { (IN.) }\end{array}$ & $\begin{array}{l}\text { CUTTER } \\
\text { INCL. } \\
\text { ANGLE } \\
\text { (DEG.) }\end{array}$ & $\begin{array}{l}\text { CUTTER } \\
\text { WEAR } \\
\text { ANGLE } \\
\text { (DEG, ) }\end{array}$ & $\begin{array}{l}\text { VOLUME } \\
\text { OF } \\
\text { CUT } \\
\text { (IN**3/ } \\
\text { REV) }\end{array}$ & $\begin{array}{l}\text { AREA E } \\
\text { OF } \\
\text { CUT } \\
\left(I^{\star \star 2} 2\right)\end{array}$ & $\begin{array}{c}\text { EFFECTIVE } \\
\text { DEPTH } \\
\text { OF CUT } \\
\text { (IN.) }\end{array}$ \\
\hline 1 & 20 & 0.665 & 0.209 & 0.988 & -15.000 & -22.557 & 0.0309 & 0.0198 & 0.0708 \\
\hline 2 & 8 & 0.776 & 0.694 & 1.113 & -10.000 & -16.561 & 0.0814 & 0.0180 & 0.0639 \\
\hline 3 & 14 & 0.923 & 1.120 & 1.252 & -5.000 & -19.724 & 0.1129 & 0.0157 & 0.0577 \\
\hline 4. & 18 & 1.035 & 1.550 & 1.384 & -2.000 & -7.433 & 0.1741 & 0.0178 & 0.0632 \\
\hline 5 & 4 & 1.008 & 1.959 & 1.361 & 0.000 & 1.249 & 0.1714 & 0.0139 & 0.0523 \\
\hline 6 & 11 & 1.022 & 2.282 & 1.374 & 2.000 & -2.397 & 0.1639 & 0.0115 & 0.0461 \\
\hline 7 & 21 & 1.030 & 2.535 & 1.380 & 5.000 & 6.287 & 0.1362 & 0.0086 & 0.0380 \\
\hline 8 & 6 & 0.983 & 2.715 & 1.327 & 10.000 & 12.699 & 0.1102 & 0.0065 & 0.0316 \\
\hline 9 & 13 & 0.967 & 2.875 & 1.305 & 15.000 & 16.308 & 0.1114 & 0.0062 & 0.0308 \\
\hline 10 & 2 & 0.902 & 3.039 & 1.232 & 20.000 & 20.564 & 0.1325 & 0.0070 & 0.0330 \\
\hline 11 & 9 & 0.862 & 3.209 & 1.179 & 25.000 & 25.818 & 0.1325 & 0.0066 & 0.0320 \\
\hline 12 & 16 & 0.807 & 3.371 & 1.112 & 30.000 & 30.004 & 0.1315 & 0.0062 & 0.0308 \\
\hline 13 & 3 & 0.702 & 3.535 & 0.992 & 35.000 & 34.714 & 0.1503 & 0.0068 & 0.0325 \\
\hline 14 & 10 & 0.615 & 3.704 & 0.883 & 40.000 & 40.496 & 0.1507 & 0.0055 & 0.0315 \\
\hline 15 & 17 & 0.507 & 3.871 & 0.750 & 45.000 & 46.356 & 0.1586 & 0.0065 & 0.0315 \\
\hline 16 & 19 & 0.385 & 4.013 & 0.602 & 47.500 & 51.510 & 0.1230 & 0.0049 & 0.0262 \\
\hline 17 & 5 & 0.233 & 4.127 & 0.431 & 50.000 & 55.127 & 0.1115 & 0.0043 & 0.0240 \\
\hline 18 & 12 & 0.128 & 4.213 & 0.317 & 52.500 & 57.099 & 0.0732 & 0.0028 & 0.0183 \\
\hline 19 & 1 & 0.003 & 4.301 & 0.140 & 55.000 & 65.787 & 0.0344 & 0.0013 & 0.0120 \\
\hline 20 & 7 & 0.016 & 4.295 & 0.165 & 55.000 & 63.848 & 0.0403 & 0.0015 & 0.0138 \\
\hline 21 & 15 & 0.029 & 4.305 & 0.156 & 55.000 & 67.449 & 0.0303 & 0.0011 & 0.0107 \\
\hline
\end{tabular}

ROCK-DEPENDENT RESULTS FOR PENETRATION RATE $=20.00 \mathrm{FT} / \mathrm{HR}$

\begin{tabular}{|c|c|c|c|c|c|c|c|c|c|}
\hline CUTTER & $\begin{array}{c}\text { VOLUME } \\
\text { OF CUI } \\
\text { (IN**3/REV) }\end{array}$ & $\begin{array}{l}\text { AREA } \\
\text { OF CUT } \\
(I N * 2)\end{array}$ & $\begin{array}{l}\text { EFFECTIVE } \\
\text { DEPTH OF CUT } \\
\text { (IN.) }\end{array}$ & $\begin{array}{l}\text { PENETRATING } \\
\text { FORCE } \\
\text { (LBF) }\end{array}$ & $\begin{array}{l}\text { DRAG } \\
\text { FORCE } \\
\text { (LBF) }\end{array}$ & $\begin{array}{l}\text { VERTICAL } \\
\text { FORCE } \\
\text { (LBF) }\end{array}$ & $\begin{array}{l}\text { RADIAL } \\
\text { FORCE } \\
\text { (LBE) }\end{array}$ & $\begin{array}{l}\text { WEARFLAT } \\
\text { TEMPERATURE } \\
\text { (DEG. C) }\end{array}$ & $\begin{array}{l}\text { WEAR } \\
\text { RATIO }\end{array}$ \\
\hline 1 & 0.0309 & 0.0198 & 0.0708 & 331.8 & 248.9 & -306.5 & 127.3 & 26.7 & 1.00 \\
\hline 2 & 0.0814 & 0.0180 & 0.0639 & 280.2 & 210.2 & -268.6 & 79.9 & 26.7 & 2.80 \\
\hline 3 & 0.1129 & 0.0157 & 0.0577 & 237.3 & 178.0 & -223.4 & 80.1 & 26.7 & 3.83 \\
\hline 4 & 0.1741 & 0.0178 & 0.0632 & 275.1 & 206.3 & -272.8 & 35.6 & 26.7 & 6.15 \\
\hline 5 & 0.1714 & 0.0139 & 0.0523 & 202.1 & 151.5 & -202.0 & -4.4 & 26.7 & 5.70 \\
\hline 6 & 0.1639 & 0.0115 & 0.0461 & 164.1 & 123.0 & -163.9 & 6.9 & 26.7 & 5.40 \\
\hline 7 & 0.1362 & 0.0086 & 0.0380 & 119.5 & 89.7 & -118.8 & -13.1 & 26.7 & 4.37 \\
\hline 8 & 0.1102 & 0.0065 & 0.0316 & 88.4 & 66.3 & -86.3 & -19.4 & 26.7 & 3.46 \\
\hline 9 & 0.1114 & 0.0062 & 0.0308 & 84.5 & 63.4 & -81.1 & -23.7 & 26.7 & 3.50 \\
\hline 10 & 0.1325 & 0.0070 & 0.0330 & 94.8 & 71.1 & -88.7 & -33.3 & 26.7 & 4.15 \\
\hline 11 & 0.1325 & 0.0066 & 0.0320 & 90.2 & 67.6 & -81.2 & -39.3 & 26.7 & 4.17 \\
\hline 12 & 0.1315 & 0.0062 & 0.0308 & 84.9 & 63.7 & -73.5 & -42.5 & 26.7 & 4.13 \\
\hline 13 & 0.1503 & 0.0068 & 0.0325 & 92.6 & 69.5 & -76.1 & -52.7 & 26.7 & 4.72 \\
\hline 14 & 0.1507 & 0.0065 & 0.0315 & 88.1 & 66.0 & -67.0 & -57.2 & 26.7 & 4.70 \\
\hline 15 & 0.1586 & 0.0065 & 0.0315 & 87.9 & 65.9 & -60.7 & -63.6 & 26.7 & 4.91 \\
\hline 16 & 0.1230 & 0.0049 & 0.0262 & 64.8 & 48.6 & -40.3 & -50.7 & 26.7 & 3.75 \\
\hline 17 & 0.1115 & 0.0043 & 0.0240 & 56.4 & 42.3 & -32.2 & -46.3 & 26.7 & 3.35 \\
\hline 18 & 0.0732 & 0.0028 & 0.0183 & 36.0 & 27.0 & -19.6 & -30.2 & 26.7 & 2.19 \\
\hline 19 & 0.0344 & 0.0013 & 0.0120 & 18.1 & 13.6 & -7.4 & -16.5 & 26.7 & 1.12 \\
\hline 20 & 0.0403 & 0.0015 & 0.0138 & 22.7 & 17.0 & -10.0 & -20.4 & 26.7 & 1.41 \\
\hline 21 & 0.0303 & 0.0011 & 0.0107 & 14.9 & 11.2 & -5.7 & -13.8 & 26.7 & 0.93 \\
\hline
\end{tabular}

INTEGRATED FORCES AND MOMENTS FOR THE FULL BIT:

$\begin{array}{llr}\text { SIERRA WHITE GRANITE AT ROP } & & 20.0 \\ \text { TOTAL WOB (LBF) } & =2285.8 \\ \text { DRILLING TORQ. (FT-LBF) } & =328.4 \\ \text { X SIDE FORCE (LBF) } & = & -5.8 \\ \text { Y SIDE FORCE (LBF) } & -179.6 \\ \text { RESULTANT SIDE FORCE (LBF) } & =179.6 \\ \text { B. M. ABOUT X-AXIS (FT-LBF) } & = & 21.6 \\ \text { B. M. ABOUT Y-AXIS (FT-LBF) } & = & -3.3\end{array}$


GEOMETRY-DEPENDENT RESULTS FOR PENETRATION RATE $=30.00 \mathrm{FT} / \mathrm{HR}$

\begin{tabular}{|c|c|c|c|c|c|c|c|c|c|}
\hline CUTTER & $\begin{array}{l}\text { CUTTING } \\
\text { ORDER }\end{array}$ & $\begin{array}{r}\text { CUTTING } \\
\text { HEIGHT } \\
\text { (IN.) }\end{array}$ & $\begin{array}{l}\text { WEARFLAT } \\
\text { LOCATION } \\
\text { XBC } \\
\text { (IN.) }\end{array}$ & $\begin{array}{l}\text { WEARFLAT } \\
\text { LOCATION } \\
\text { ZBC } \\
\text { (IN.) }\end{array}$ & $\begin{array}{l}\text { CUTTER } \\
\text { INCL. } \\
\text { ANGLE } \\
\text { (DEG.) }\end{array}$ & $\begin{array}{l}\text { CUTTER } \\
\text { WEAR } \\
\text { ANGLE } \\
\text { (DEG. ) }\end{array}$ & $\begin{array}{l}\text { VOLUME } \\
\text { OF } \\
\text { CUT } \\
\text { (IN" }{ }^{*} 3 / \\
\text { REV ) }\end{array}$ & $\begin{array}{l}\text { AREA } \\
\text { OF } \\
\text { CUT } \\
\left(I N^{* * 2}\right)\end{array}$ & $\begin{array}{c}\text { EFFECTIVE } \\
\text { DEPTH } \\
\text { OF CUT } \\
\text { (IN.) }\end{array}$ \\
\hline 1 & 20 & 0.685 & 0.213 & 1.010 & -15.000 & -21.959 & 0.0465 & 0.0298 & 0.0893 \\
\hline 2 & 8 & 0.784 & 0.697 & 1. 121 & -10.000 & -16.169 & 0.1228 & 0.0271 & 0.0825 \\
\hline 3 & 14 & 0.937 & 1.124 & 1.267 & -5.000 & -19.090 & 0.1714 & 0.0238 & 0.0756 \\
\hline 4 & 18 & 1.053 & 1.557 & 1.402 & -2.000 & -6.463 & 0.2605 & 0.0265 & 0.0812 \\
\hline 5 & 4 & 1.013 & 1.961 & 1. 365 & 0.000 & 1.593 & 0.2575 & 0.0209 & 0.0695 \\
\hline 6 & 11 & 1.033 & 2.290 & 1.385 & 2.000 & -1.221 & 0.2446 & 0.0171 & 0.0609 \\
\hline 7 & 21 & 1.050 & 2. 542 & $1: 399$ & .5 .000 & 7.424 & 0.2109 & 0.0133 & 0.0509 \\
\hline 8 & 6 & 0.990 & 2.723 & 1.331 & 10.000 & 14.003 & 0.1650 & 0.0097 & 0.0411 \\
\hline 9 & 13 & 0.980 & 2.880 & 1.317 & 15.000 & 17.011 & 0.1610 & 0.0089 & 0.0391 \\
\hline 10 & 2 & 0.905 & 3.043 & 1.233 & 20.000 & 21.201 & 0.2039 & 0.0107 & 0.0439 \\
\hline 11 & 9 & 0.871 & 3.215 & 1.185 & 25.000 & 26.757 & 0.1981 & 0.0098 & 0.0418 \\
\hline 12 & 16 & 0.823 & 3.375 & 1.126 & 30.000 & 30.639 & 0.1931 & 0.0091 & 0.0399 \\
\hline 13 & 3 & 0.706 & 3.539 & 0.993 & 35.000 & 35.336 & 0.2280 & 0.0103 & 0.0430 \\
\hline 14 & 10 & 0.626 & 3.709 & 0.889 & 40.000 & 41.413 & 0.2252 & 0.0097 & 0.0414 \\
\hline 15 & 17 & 0.525 & 3.875 & 0.762 & 45.000 & 47.300 & 0.2423 & 0.0100 & 0.0422 \\
\hline 16 & 19 & 0.404 & 4.016 & 0.616 & 47.500 & 52.328 & 0.1790 & 0.0071 & 0.0344 \\
\hline 17 & 5 & 0.239 & 4.128 & 0.435 & 50.000 & 55.407 & 0.1668 & 0.0064 & 0.0319 \\
\hline 18 & 12 & 0.141 & 4.210 & 0.333 & 52.500 & 56.363 & 0.0931 & 0.0035 & 0.0217 \\
\hline 19 & 1 & 0.004 & 4.297 & 0.149 & 55.000 & 64.607 & 0.0554 & 0.0021 & 0.0155 \\
\hline 20 & 7 & 0.024 & 4.287 & 0.189 & 55.000 & 61.325 & 0.0719 & 0.0027 & 0.0191 \\
\hline 21 & 15 & 0.044 & 4.305 & 0.171 & 55.000 & 67.449 & 0.0452 & 0.0017 & 0.0130 \\
\hline
\end{tabular}

ROCK-DEPENDENT RESULTS FOR PENETRATION RATE $=30.00 \mathrm{FT} / \mathrm{HR}$

\begin{tabular}{|c|c|c|c|c|c|c|c|c|c|}
\hline CUTTER & $\begin{array}{c}\text { VOLUME } \\
\text { OF CUT } \\
\text { (IN**3/REV) }\end{array}$ & $\begin{array}{l}\text { AREA } \\
\text { OF CUT } \\
(I N * \star 2)\end{array}$ & $\begin{array}{l}\text { EFFECTIVE } \\
\text { DEPTH OF CUT } \\
\text { (IN : ) }\end{array}$ & $\begin{array}{c}\text { PENETRATING } \\
\text { FORCE } \\
\text { (LBF) }\end{array}$ & $\begin{array}{l}\text { DRAG } \\
\text { FORCE } \\
\text { (LBF) }\end{array}$ & $\begin{array}{l}\text { VERTICAL } \\
\text { FORCE } \\
\text { (LBE) }\end{array}$ & $\begin{array}{l}\text { RADIAL } \\
\text { FORCE } \\
\text { (LBF) }\end{array}$ & $\begin{array}{l}\text { WEARFLAT } \\
\text { TEMPERATURE } \\
\text { (DEG. C) }\end{array}$ & $\begin{array}{l}\text { WEAR } \\
\text { RATIO }\end{array}$ \\
\hline 1 & 0.0465 & 0.0298 & 0.0893 & 485.1 & 363.8 & -449.9 & 181.4 & 26.7 & 1.00 \\
\hline 2 & 0.1228 & 0.0271 & 0.0825 & 426.2 & 319.6 & -409.3 & 118.7 & 26.7 & 2.88 \\
\hline 3 & 0.1714 & 0.0238 & 0.0756 & 369.2 & 276.9 & -348.9 & 120.8 & 26.7 & 4.02 \\
\hline 4 & 0.2605 & 0.0265 & 0.0812 & 414.9 & 311.2 & -412.3 & 46.7 & 26.7 & 6.25 \\
\hline 5 & 0.2575 & 0.0209 & 0.0695 & 321.5 & 241.1 & -321.4 & -8.9 & 26.7 & 6.10 \\
\hline 6 & 0.2446 & 0.0171 & 0.0609 & 258.7 & 194.0 & -258.7 & 5.5 & 26.7 & 5.74 \\
\hline 7 & 0.2109 & 0.0133 & 0.0509 & .192 .9 & 144.7 & -191.3 & -24.9 & 26.7 & 4.75 \\
\hline 8 & 0.1650 & 0.0097 & 0.0411 & 135.9 & 101.9 & -131.8 & -32.9 & 26.7 & 3.58 \\
\hline 9 & 0.1610 & 0.0089 & 0.0391 & 125.0 & 93.8 & -119.6 &.-36.6 & 26.7 & 3.49 \\
\hline 10 & 0.2039 & 0.0107 & 0.0439 & 151.6 & 113.7 & -141.3 & -54.8 & 26.7 & 4.47 \\
\hline 11 & 0.1981 & 0.0098 & 0.0418 & 140.0 & 105.0 & -125.0 & -63.0 & 26.7 & 4.36 \\
\hline 12 & 0.1931 & 0.0091 & 0.0399 & 129.4 & 97.1 & -111.4 & -66.0 & 26.7 & 4.23 \\
\hline 13 & 0.2280 & 0.0103 & 0.0430 & 146.2 & 109.7 & -119.3 & -84.6 & 26.7 & 5.01 \\
\hline 14 & 0.2252 & 0.0097 & 0.0414 & 137.8 & 103.3 & -103.3 & -91.1 & 26.7 & 4.95 \\
\hline 15 & 0.2423 & 0.0100 & 0.0422 & 142.0 & 106.5 & -96.3 & -104.3 & 26.7 & 5.33 \\
\hline 16 & 0.1790 & 0.0071 & 0.0344 & 101.5 & 76.1 & -62.0 & -80.3 & 26.7 & 3.95 \\
\hline 17 & 0.1668 & 0.0064 & 0.0319 & 89.5 & 67.1 & -50.8 & -73.7 & 26.7 & 3.58 \\
\hline 18 & 0.0931 & 0.0035 & 0.0217 & 47.8 & 35.8 & -26.5 & -39.8 & 26.7 & 1.95 \\
\hline 19 & 0.0554 & 0.0021 & 0.0155 & 27.4 & 20.5 & -11.7 & -24.7 & 26.7 & 1.14 \\
\hline 20 & 0.0719 & 0.0027 & 0.0191 & 38.7 & 29.0 & -18.6 & -33.9 & 26.7 & 1.61 \\
\hline 21 & $0: 0452$ & 0.0017 & 0.0130 & 20.7 & 15.5 & -7.9 & -19.1 & 26.7 & 0.86 \\
\hline
\end{tabular}

INTEGRATED FORCES AND MOMENTS FOR THE FULL BIT:

SIERRA WHITE GRANITE AT ROP $=30.0$

$\begin{array}{llr}\text { TOTAL WOB (LBF) } & =3517.3 \\ \text { DRILLING TORQ. (FT-LBF) } & =511.4 \\ \text { X SIDE FORCE (LBF) } & =-11.0 \\ \text { Y SIDE FORCE (LBF) } & =-275.8 \\ \text { RESULTANT SIDE FORCE (LBF) }= & 276.0 \\ \text { B. M. ABOUT X-AXIS (FT-LBF) }= & 27.2 \\ \text { B. M. ABOUT Y-AXIS (FT-LBF) }= & -2.5\end{array}$


GEOMETRY-DEPENDENT RESULTS FOR PENETRATION RATE $=40.00 \mathrm{FT} / \mathrm{HR}$

\begin{tabular}{|c|c|c|c|c|c|c|c|c|c|}
\hline CUTTER & $\begin{array}{l}\text { CUTTING } \\
\text { ORDER }\end{array}$ & $\begin{array}{r}\text { CUTTING } \\
\text { HEIGHT } \\
\text { (IN.) }\end{array}$ & $\begin{array}{l}\text { WEARFIAT } \\
\text { LOCATION } \\
\text { XBC } \\
\text { (IN.) }\end{array}$ & $\begin{array}{l}\text { WEARFLAT } \\
\text { LOCATION } \\
\text { ZBC } \\
\text { (IN.) }\end{array}$ & $\begin{array}{l}\text { CUTTER } \\
\text { INCL. } \\
\text { ANGLE } \\
\text { (DEG. ) }\end{array}$ & $\begin{array}{l}\text { CUTTER } \\
\text { WEAR } \\
\text { ANGLE } \\
\text { (DEG. ) }\end{array}$ & $\begin{array}{l}\text { VOLUME } \\
\text { OF } \\
\text { CUT } \\
\text { (IN**3/ } \\
\text { REV ) }\end{array}$ & $\begin{array}{l}\text { AREA } \\
\text { OF } \\
\text { CUT } \\
\left(I N^{\star *} 2\right)\end{array}$ & $\begin{array}{c}\text { EFFECTIVE } \\
\text { DEPTH } \\
\text { OF CUT } \\
\text { (IN.) }\end{array}$ \\
\hline 1 & 20 & 0.705 & 0.217 & 1.032 & -15.000 & -21.355 & 0.0624 & 0.0399 & 0.1075 \\
\hline 2 & 8 & 0.792 & 0.698 & 1.130 & -10.000 & -16.084 & 0.1646 & 0.0362 & 0.1004 \\
\hline 3 & 14 & 0.951 & 1.128 & 1.283 & -5.000 & -18.456 & 0.2314 & 0.0320 & 0.0927 \\
\hline 4 & 18 & 1.070 & 1.563 & 1.421 & -2.000 & -5.535 & 0.3462 & 0.0351 & 0.0983 \\
\hline 5 & 4 & 1.017 & 1.966 & 1.369 & 0.000 & 2.288 & 0.3440 & 0.0279 & 0.0852 \\
\hline 6 & 11 & 1.043 & 2.296 & 1.396 & 2.000 & -0.304 & 0.3244 & 0.0226 & 0.0740 \\
\hline 7 & 21 & 1.070 & 2.541 & 1.419 & 5.000 & 7.267 & 0.2863 & 0.0180 & 0.0628 \\
\hline 8 & 6 & 0.997 & 2.716 & 1.340 & 10.000 & 12.966 & 0.2177 & 0.0127 & 0.0497 \\
\hline 9 & 13 & 0.993 & 2.883 & 1.329 & 15.000 & 17.469 & 0.2116 & 0.0117 & 0.0467 \\
\hline 10 & 2 & 0.907 & 3.044 & 1.235 & 20.000 & 21.340 & 0.2781 & 0.0146 & 0.0540 \\
\hline 11 & 9 & 0.880 & 3.220 & 1.191 & 25.000 & 27.626 & 0.2631 & 0.0130 & 0.0503 \\
\hline 12 & 16 & 0.838 & 3.377 & 1.140 & 30.000 & 30.948 & 0.2522 & 0.0119 & 0.0476 \\
\hline 13 & 3 & 0.710 & 3.541 & 0.996 & 35.000 & 35.647 & 0.3073 & 0.0139 & 0.0524 \\
\hline 14 & 10 & 0.636 & 3.711 & 0.898 & 40.000 & 41.895 & 0.2992 & 0.0129 & 0.0503 \\
\hline 15 & 17 & 0.542 & 3.880 & 0.775 & 45.000 & 48.259 & 0.3282 & 0.0135 & 0.0518 \\
\hline 16 & 19 & 0.423 & 4.023 & 0.626 & 47.500 & 53.909 & 0.2319 & 0.0092 & 0.0407 \\
\hline 17 & 5 & 0.244 & 4.128 & 0.440 & 50.000 & 55.507 & 0.2216 & 0.0086 & 0.0385 \\
\hline 18 & 12 & 0.153 & 4.211 & 0.345 & 52.500 & 56.502 & 0.1093 & 0.0041 & 0.0238 \\
\hline 19 & 1 & 0.006 & 4.293 & 0.159 & 55.000 & 63.107 & 0.0795 & 0.0030 & 0.0192 \\
\hline 20 & 7 & 0.032 & 4.287 & 0.197 & 55.000 & 61.325 & 0.1040 & 0.0039 & 0.0229 \\
\hline 21 & 15 & 0.059 & 4.304 & 0.188 & 55.000 & 67.023 & 0.0600 & 0.0022 & 0.0154 \\
\hline
\end{tabular}

ROCK-DEPENDENT RESULTS FOR PENETRATION RATE $=40.00 \mathrm{FT} / \mathrm{HR}$

\begin{tabular}{|c|c|c|c|c|c|c|c|c|c|}
\hline CUTTER & $\begin{array}{c}\text { VOLUME } \\
\text { OF CUT } \\
\text { (IN**3/REV) }\end{array}$ & $\begin{array}{l}\text { AREA } \\
\text { OF CUT } \\
\left(I N^{\star \star 2}\right)\end{array}$ & $\begin{array}{l}\text { EFEECTIVE } \\
\text { DEPTH OF CUT } \\
\text { (IN.) }\end{array}$ & $\begin{array}{c}\text { PENETRATING } \\
\text { FORCE } \\
\text { (LBF) }\end{array}$ & $\begin{array}{l}\text { DRAG } \\
\text { FORCE } \\
\text { (LBF) }\end{array}$ & $\begin{array}{l}\text { VERTICAL } \\
\text { FORCE } \\
\text { (LBF) }\end{array}$ & $\begin{array}{l}\text { RADIAL } \\
\text { FORCE } \\
\text { (LBE) }\end{array}$ & $\begin{array}{l}\text { WEARFLAT } \\
\text { TEMPERATURE } \\
\text { (DEG. C) }\end{array}$ & $\begin{array}{l}\text { WEAR } \\
\text { RATIO }\end{array}$ \\
\hline 1 & 0.0624 & 0.0399 & 0.1075 & 657.7 & 493.3 & -612.6 & 239.5 & 26.7 & 1.00 \\
\hline 2 & 0.1646 & 0.0362 & 0.1004 & 587.8 & 440.9 & -564.8 & 162.9 & 26.7 & 2.88 \\
\hline 3 & 0.2314 & 0.0320 & 0.0927 & 515.4 & 386.6 & -488.9 & 163.2 & 26.7 & 4.08 \\
\hline 4 & 0.3462 & 0.0351 & 0.0983 & 567.5 & 425.6 & -564.9 & 54.7 & 26.7 & 6.22 \\
\hline 5 & 0.3440 & 0.0279 & 0.0852 & 448.9 & 336.7 & -448.5 & -17.9 & 26.7 & 6.19 \\
\hline 6 & 0.3244 & 0.0226 & 0.0740 & 356.7 & 267.5 & -356.7 & 1.9 & 26.7 & 5.74 \\
\hline 7 & 0.2863 & 0.0180 & 0.0628 & 272.5 & 204.4 & -270.3 & -34.5 & 26.7 & 4.86 \\
\hline 8 & 0.2177 & 0.0127 & 0.0497 & 185.6 & 139.2 & -180.9 & -41.6 & 26.7 & 3.54 \\
\hline 9 & 0.2116 & 0.0117 & 0.0467 & 167.4 & 125.6 & -159.7 & -50.3 & 26.7 & 3.39 \\
\hline 10 & 0.2781 & 0.0146 & 0.0540 & 212.4 & 159.3 & -197.9 & -77.3 & 26.7 & 4.54 \\
\hline 11 & 0.2631 & 0.0130 & 0.0503 & 189.0 & 141.8 & -167.5 & -87.7 & 26.7 & 4.27 \\
\hline 12 & 0.2522 & 0.0119 & 0.0476 & 172.9 & 129.7 & -148.3 & -88.9 & 26.7 & 4.10 \\
\hline 13 & 0.3073 & 0.0139 & 0.0524 & 202.6 & 152.0 & -164.7 & -118.1 & 26.7 & 5.03 \\
\hline 14 & 0.2992 & 0.0129 & 0.0503 & 189.1 & 141.8 & -140.7 & -126.3 & 26.7 & 4.92 \\
\hline 15 & 0.3282 & 0.0135 & 0.0518 & 198.3 & 148.7 & -132.0 & -148.0 & 26.7 & 5.40 \\
\hline 16 & 0.2319 & 0.0092 & 0.0407 & 133.5 & 100.1 & -78.6 & -107.9 & 26.7 & 3.77 \\
\hline 17 & 0.2216 & 0.0086 & 0.0385 & 122.1 & 91.6 & -69.1 & -100.6 & 26.7 & 3.53 \\
\hline 18 & 0.1093 & 0.0041 & 0.0238 & 55.4 & 41.6 & -30.6 & -46.2 & 26.7 & 1.64 \\
\hline 19 & 0.0795 & 0.0030 & 0.0192 & 39.1 & 29.3 & -17.7 & -34.9 & 26.7 & 1,18 \\
\hline 20 & 0.1040 & 0.0039 & 0.0229 & 52.2 & 39.2 & -25.1 & -45.8 & 26.7 & 1.57 \\
\hline 21 & 0.0600 & 0.0022 & 0.0154 & 27.3 & 20.5 & -10.7 & -25.1 & 26.7 & 0.82 \\
\hline
\end{tabular}

INTEGRATED FORCES AND MOMENTS FOR THE FULL BIT:

SIERRA WHITE GRANITE AT ROP $=40.0$

$\begin{array}{llr}\text { TOTAL WOB (LBF) } & =4830.2 \\ \text { DRILLING TORQ. (FT-LBF) } & =700.9 \\ \text { X SIDE FORCE (LBF) } & =-22.4 \\ \text { Y SIDE FORCE (LBF) } & =385.2 \\ \text { RESULTANT SIDE FORCE (LBF) } & =385.8 \\ \text { B. M. ABOUT X-AXIS (FT-LBF) } & 32.6 \\ \text { B. M. ABOUT Y-AXIS (FT-LBF) } & 0.1\end{array}$


GEOMETRY-DEPENDENT RESULTS FOR PENETRATION RATE $=50.00 \mathrm{FT} / \mathrm{HR}$

\begin{tabular}{|c|c|c|c|c|c|c|c|c|c|}
\hline CUTTER & $\begin{array}{c}\text { CUTTING } \\
\text { ORDER }\end{array}$ & $\begin{array}{r}\text { CUTTING } \\
\text { HEIGHT } \\
\text { (IN.) }\end{array}$ & $\begin{array}{l}\text { WEARFLAT } \\
\text { LOCATION } \\
\text { XBC } \\
\text { (IN.) }\end{array}$ & $\begin{array}{c}\text { WEARFLAT } \\
\text { LOCATION } \\
\text { ZBC } \\
\text { (IN.) }\end{array}$ & $\begin{array}{l}\text { CUTTER } \\
\text { INCL. } \\
\text { ANGLE } \\
\text { (DEG. ) }\end{array}$ & $\begin{array}{l}\text { CUTTER } \\
\text { WEAR } \\
\text { ANGLE } \\
\text { (DEG.) }\end{array}$ & $\begin{array}{l}\text { VOLUME } \\
\text { OF } \\
\text { CUT } \\
(\text { IN**3/ } \\
\text { REV) }\end{array}$ & $\begin{array}{l}\text { AREA } \\
\text { OF } \\
\text { CUT } \\
(I N * * 2)\end{array}$ & $\begin{array}{c}\text { EFFECTIVE } \\
\text { DEPTH } \\
\text { OF CUT } \\
\text { (IN.) }\end{array}$ \\
\hline 1 & 20 & 0.725 & 0.223 & 1.054 & -15.000 & -20.436 & 0.0785 & 0.0500 & 0.1255 \\
\hline 2 & 8 & 0.800 & 0.700 & 1.138 & -10.000 & -15.700 & 0.2070 & 0.0454 & 0.1176 \\
\hline 3 & 14 & 0.964 & 1.136 & 1.299 & -5.000 & -17.283 & 0.2928 & 0.0403 & 0.1092 \\
\hline 4 & 18 & 1.087 & 1. 567 & 1.438 & -2.000 & -4.914 & 0.4311 & 0.0436 & 0.1143 \\
\hline 5 & 4 & 1.021 & 1.971 & 1.373 & 0.000 & 2.998 & 0.4307 & 0.0349 & 0.1001 \\
\hline 6 & 11 & 1.054 & 2.305 & 1.407 & 2.000 & 0.984 & 0.4033 & 0.0281 & 0.0860 \\
\hline 7 & 21 & 1.090 & 2.536 & 1.440 & 5.000 & 6.468 & 0.3577 & 0.0224 & 0.0731 \\
\hline 8 & 6 & 1.003 & 2.712 & 1.347 & 10.000 & 12.272 & 0.2678 & 0.0157 & 0.0571 \\
\hline 9 & 13 & 1.007 & 2.879 & 1.344 & 15.000 & 16.851 & 0.2655 & 0.0147 & 0.0544 \\
\hline 10 & 2 & 0.909 & 3.039 & 1.239 & 20.000 & 20.555 & 0.3555 & 0.0186 & 0.0638 \\
\hline 11 & 9 & 0.888 & 3.215 & 1.203 & 25.000 & 26.741 & 0.3274 & 0.0162 & 0.0583 \\
\hline 12 & 26 & 0.854 & 3.378 & 1.155 & 30.000 & 31.103 & 0.3109 & 0.0147 & 0.0545 \\
\hline 13 & 3 & 0.713 & 3.539 & 1.001 & 35.000 & 35.251 & 0.3882 & 0.0175 & 0.0613 \\
\hline 14 & 10 & 0.647 & 3.713 & 0.906 & 40.000 & 42.280 & 0.3727 & 0.0160 & 0.0581 \\
\hline 15 & 17 & 0.559 & 3.887 & 0.783 & 45.000 & 49.869 & 0.4157 & 0.0171 & 0.0604 \\
\hline 16 & 19 & 0.441 & 4.028 & 0.638 & 47.500 & 55.083 & 0.2824 & 0.0112 & 0.0466 \\
\hline 17 & 5 & 0.250 & 4.131 & 0.442 & 50.000 & 56.180 & 0.2756 & 0.0107 & 0.0445 \\
\hline 18 & 12 & 0.165 & 4.210 & 0.358 & 52.500 & 56.401 & 0.1271 & 0.0048 & 0.0259 \\
\hline 19 & 1 & 0.007 & 4.286 & 0.174 & 55.000 & 60.979 & 0.1079 & 0.0040 & 0.0235 \\
\hline 20 & 7 & 0.040 & 4.287 & 0.205 & 55.000 & 61.325 & 0.1308 & 0.0049 & 0.0262 \\
\hline 21 & 15 & 0.074 & 4.303 & 0.205 & 55.000 & 66.604 & 0.0748 & 0.0028 & 0.0178 \\
\hline
\end{tabular}

ROCK-DEPENDENT RESULTS FOR PENETRATION RATE $=50.00 \mathrm{FT} / \mathrm{HR}$

\begin{tabular}{|c|c|c|c|c|c|c|c|c|c|}
\hline CUTTER & $\begin{array}{c}\text { VOLUME } \\
\text { OF CUT } \\
\text { (IN**3/REV) }\end{array}$ & $\begin{array}{l}\text { AREA } \\
\text { OF CUT } \\
\left(I N^{* * 2}\right)\end{array}$ & $\begin{array}{l}\text { EFFECIIVE } \\
\text { DEPTH OF CUT } \\
\text { (IN.) }\end{array}$ & $\begin{array}{c}\text { PENETRATING } \\
\text { FORCE } \\
\text { (LBF) }\end{array}$ & $\begin{array}{l}\text { DRAG } \\
\text { FORCE } \\
\text { (LBF) }\end{array}$ & $\begin{array}{l}\text { VERTICAI } \\
\text { FORCE } \\
\text { (LBF) }\end{array}$ & $\begin{array}{l}\text { RADIAL } \\
\text { FORCE } \\
\text { (LBF) }\end{array}$ & $\begin{array}{l}\text { WEARFLAT } \\
\text { TEMPERATURE } \\
\text { (DEG. C) }\end{array}$ & $\begin{array}{l}\text { WEAR } \\
\text { RATIO }\end{array}$ \\
\hline 1 & 0.0785 & 0.0500 & 0.1255 & 847.3 & 635.5 & -794.0 & 295.8 & 26.7 & 1.00 \\
\hline 2 & 0.2070 & 0.0454 & 0.1176 & 762.2 & 571.7 & -733.8 & 206.3 & 26.7 & 2.83 \\
\hline 3 & 0.2928 & 0.0403 & 0.1092 & 674.8 & 506.1 & -644.3 & 200.5 & 26.7 & 4.06 \\
\hline 4 & 0.4311 & 0.0436 & 0.1143 & 727.1 & 545.3 & -724.5 & 62.3 & 26.7 & 6.04 \\
\hline 5 & 0.4307 & 0.0349 & 0.1001 & 585.5 & 439.2 & -584.7 & -30.6 & 26.7 & 6.11 \\
\hline 6 & 0.4033 & 0.0281 & 0.0860 & 456.6 & 342.4 & -456.5 & -7.8 & 26.7 & 5.58 \\
\hline 7 & 0.3577 & 0.0224 & 0.0731 & 349.7 & 262.3 & -347.5 & -39.4 & 26.7 & 4.70 \\
\hline 8 & 0.2678 & 0.0157 & 0.0571 & 233.1 & 174.9 & -227.8 & -49.6 & 26.7 & 3.35 \\
\hline 9 & 0.2655 & 0.0147 & 0.0544 & 215.3 & 161.4 & -206.0 & -62.4 & 26.7 & 3.28 \\
\hline 10 & 0.3555 & 0.0186 & 0.0638 & 279.2 & 209.4 & -261.4 & -98.0 & 26.7 & 4.50 \\
\hline 11 & 0.3274 & 0.0162 & 0.0583 & 241.3 & 180.9 & -215.5 & -108.6 & 26.7 & 4.11 \\
\hline 12 & 0.3109 & 0.0147 & 0.0545 & 215.8 & 161.9 & -184.8 & -111.5 & 26.7 & 3.86 \\
\hline 13 & 0.3882 & 0.0175 & 0.0613 & 261.7 & 196.2 & -213.7 & -151.0 & 26.7 & 4.91 \\
\hline 14 & 0.3727 & 0.0160 & 0.0581 & 239.5 & 179.6 & -177.2 & -161.1 & 26.7 & 4.71 \\
\hline 15 & 0.4157 & 0.0171 & 0.0604 & 255.6 & 191.7 & -164.7 & -195.4 & 26.7 & 5.26 \\
\hline 16 & 0.2824 & 0.0112 & 0.0466 & 167.1 & 125.3 & -95.6 & -137.0 & 26.7 & 3.57 \\
\hline 17 & 0.2756 & 0.0107 & 0.0445 & 154.6 & 115.9 & -86.0 & -128.4 & 26.7 & 3.38 \\
\hline 18 & 0.1271 & 0.0048 & 0.0259 & 63.7 & 47.8 & -35.3 & -53.1 & 26.7 & 1. 42 \\
\hline 19 & 0.1079 & 0.0040 & 0.0235 & 54.3 & 40.7 & -26.4 & -47.5 & 26.7 & 1.23 \\
\hline 20 & 0.1308 & 0.0049 & 0.0262 & 64.8 & 48.6 & -31.1 & -56.8 & 26.7 & 1.47 \\
\hline 21 & 0.0748 & 0.0028 & 0.0178 & 34.3 & 25.7 & -13.6 & -31.5 & 26.7 & 0.78 \\
\hline
\end{tabular}

INTEGRATED FORCES AND MOMENTS FOR THE FULL BIT:

SIERRA WHITE GRANITE AT ROP $=50.0$

$\begin{array}{llr}\text { TOTAL WOB (LBF) } & =6224.4 \\ \text { DRILLING TORQ. (FT-LBF) } & =898.4 \\ \text { X SIDE FORCE (LBF) } & =-34.7 \\ \text { Y SIDE FORCE (LBF) } & -496.5 \\ \text { RESULTANT SIDE FORCE (LBF) } & 497.7 \\ \text { B. M. ABOUT X-AXIS (FT-LBF) } & 36.9 \\ \text { B. M. ABOUT Y-AXIS (FT-LBF) } & 3.3\end{array}$


INTEGRATED AND SUMMARY DATA FOR THE FULL BIT AT ALI SPECIFIED PENETRATION RATES * :

\begin{tabular}{lrrrrrr} 
SIERRA WHITE GRANITE AT ROP $=$ & 10.0 & 20.0 & 30.0 & 40.0 & 50.0 \\
\hline TOTAL WOB (LBF) & $=$ & 1171.1 & 2285.8 & 3517.3 & 4830.2 & 6224.4 \\
DRILLING TORQ. (FT-LBF) & $=$ & 159.1 & 328.4 & 511.4 & 700.9 & 898.4 \\
X SIDE FORCE (LBF) & $=$ & 1.4 & -5.8 & -11.0 & -22.4 & -34.7 \\
Y SIDE FORCE (LBF) & $=$ & -109.0 & -179.6 & -275.8 & -385.2 & -496.5 \\
RESULTANT SIDE FORCE (LBF) & $=$ & 109.0 & 179.6 & 276.0 & 385.8 & 497.7 \\
B. M. ABOUT X-AXIS (FT-LBF) $=$ & 15.3 & 21.6 & 27.2 & 32.6 & 36.9 \\
B. M. ABOUT Y-AXIS (FT-LBF) $=$ & -1.2 & -3.3 & -2.5 & 0.1 & 3.3 \\
MAX. WEARFLAT TEMP. (DEG. C) & $=$ & 26.7 & 26.7 & 26.7 & 26.7 & 26.7 \\
MAX. WEAR RATIO & $=$ & 5.64 & 6.15 & 6.25 & 6.22 & 6.11
\end{tabular}

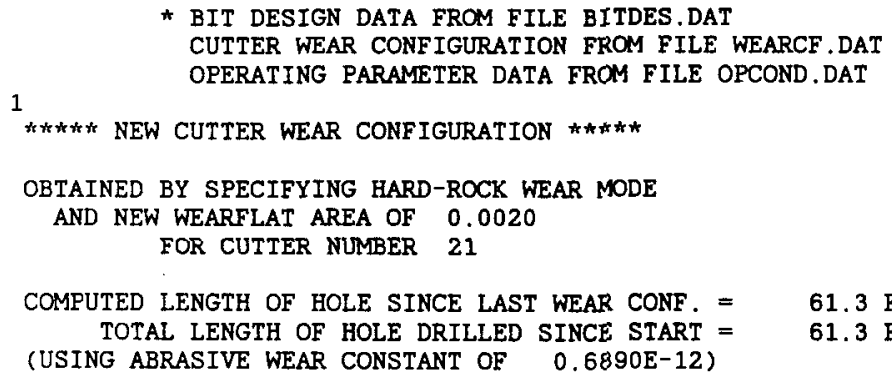

(THIS CONFIGURATION WAS STORED IN DISK FILE WCBOO2.DAT )

$\begin{array}{cccc}\text { CUTTER } & \begin{array}{c}\text { WEARFLAT } \\ \text { LENGTH } \\ \text { (IN.) }\end{array} & \begin{array}{c}\text { WEARFLAT } \\ \text { WIDTH } \\ \text { (IN.) }\end{array} & \begin{array}{c}\text { WEARFLAT } \\ \text { ARFA } \\ \text { (IN**2) }\end{array} \\ 1 & 0.021 & 0.147 & 0.0022 \\ 2 & 0.033 & 0.182 & 0.0041 \\ 3 & 0.037 & 0.194 & 0.0049 \\ 4 & 0.045 & 0.212 & 0.0064 \\ 5 & 0.044 & 0.211 & 0.0063 \\ 6 & 0.043 & 0.208 & 0.0061 \\ 7 & 0.040 & 0.200 & 0.0055 \\ 8 & 0.036 & 0.190 & 0.0046 \\ 9 & 0.035 & 0.189 & 0.0045 \\ 10 & 0.039 & 0.198 & 0.0053 \\ 11 & 0.039 & 0.197 & 0.0052 \\ 12 & 0.038 & 0.196 & 0.0051 \\ 13 & 0.041 & 0.203 & 0.0056 \\ 14 & 0.041 & 0.202 & 0.0056 \\ 15 & 0.042 & 0.205 & 0.0058 \\ 16 & 0.037 & 0.193 & 0.0049 \\ 17 & 0.036 & 0.190 & 0.0046 \\ 18 & 0.028 & 0.168 & 0.0032 \\ 19 & 0.023 & 0.151 & 0.0024 \\ 20 & 0.026 & 0.162 & 0.0029 \\ 21 & 0.020 & 0.143 & 0.0020\end{array}$




\begin{tabular}{|c|c|c|c|c|c|c|c|c|c|}
\hline CUTTER & $\begin{array}{l}\text { CUTTING } \\
\text { ORDER }\end{array}$ & $\begin{array}{r}\text { CUTTING } \\
\text { BEIGHT } \\
\text { (IN.) }\end{array}$ & $\begin{array}{l}\text { WEARFLAT } \\
\text { LOCATION } \\
\text { XBC } \\
\text { (IN.) }\end{array}$ & $\begin{array}{l}\text { WEARFLAT } \\
\text { LOCATION } \\
\text { ZBC } \\
\text { (IN.) }\end{array}$ & $\begin{array}{l}\text { CUTTER } \\
\text { INCL. } \\
\text { ANGLE } \\
\text { (DEG.) }\end{array}$ & $\begin{array}{l}\text { CUTTER } \\
\text { WEAR } \\
\text { ANGLE } \\
\text { (DEG.) }\end{array}$ & $\begin{array}{l}\text { VOLUME } \\
\text { OF } \\
\text { CUT } \\
\text { (IN**3/ } \\
\text { REV ) }\end{array}$ & $\begin{array}{l}\text { AREA } \\
\text { OF } \\
\text { CUT } \\
\left(I N^{*} 2\right)\end{array}$ & $\begin{array}{c}\text { EFFECTIVE } \\
\text { DEPTH } \\
\text { OF CUT } \\
\text { (IN.) }\end{array}$ \\
\hline 1 & 20 & 0.645 & 0.208 & 0.961 & -15.000 & -23.149 & 0.0153 & 0.0099 & 0.0465 \\
\hline 2 & 8 & 0.768 & 0.697 & 1.094 & -10.000 & -16.660 & 0.0405 & 0.0090 & 0.0368 \\
\hline 3 & 14 & 0.909 & 1.120 & 1.226 & -5.000 & -20.358 & 0.0558 & 0.0078 & 0.0305 \\
\hline 4 & 18 & 1.018 & 1.546 & 1.352 & -2.000 & -8.296 & 0.0873 & 0.0089 & 0.0337 \\
\hline 5 & 4 & 1.004 & 1.954 & 1.342 & 0.000 & 0.609 & 0.0856 & 0.0070 & 0.0249 \\
\hline 6 & 11 & 1.011 & 2.277 & 1.349 & 2.000 & -3.241 & 0.0823 & 0.0058 & 0.0212 \\
\hline 7 & 21 & 1.010 & 2.538 & 1.347 & 5.000 & 7.073 & 0.0649 & 0.0041 & 0.0163 \\
\hline 8 & 6 & 0.977 & 2.701 & 1.311 & 10.000 & 10.963 & 0.0557 & 0.0033 & 0.0138 \\
\hline 9 & 13 & 0.953 & 2.866 & 1.282 & 15.000 & 15.493 & 0.0575 & 0.0032 & 0.0135 \\
\hline 10 & 2 & 0.900 & 3.029 & 1.221 & 20.000 & 19.574 & 0.0642 & 0.0034 & 0.0138 \\
\hline 11 & 9 & 0.853 & 3.196 & 1.162 & 25.000 & 24.615 & 0.0666 & 0.0033 & 0.0137 \\
\hline 12 & 16 & 0.792 & 3.358 & 1.090 & 30.000 & 28.765 & 0.0669 & 0.0032 & 0.0132 \\
\hline 13 & 3 & 0.698 & 3.520 & 0.982 & 35.000 & 33.225 & 0.0750 & 0.0034 & 0.0137 \\
\hline 14 & 10 & 0.605 & 3.686 & 0.870 & 40.000 & 38.614 & 0.0755 & 0.0033 & 0.0134 \\
\hline 15 & 17 & 0.490 & 3.852 & 0.733 & 45.000 & 44.376 & 0.0779 & 0.0032 & 0.0131 \\
\hline 16 & 19 & 0.366 & 3.996 & 0.585 & 47.500 & 49.722 & 0.0621 & 0.0025 & 0.0110 \\
\hline 17 & 5 & 0.228 & 4.113 & 0.425 & 50.000 & 54.113 & 0.0576 & 0.0022 & 0.0099 \\
\hline 18 & 12 & 0.116 & 4.198 & 0.310 & 52.500 & 55.213 & 0.0370 & 0.0014 & 0.0073 \\
\hline 19 & 1 & 0.001 & 4.294 & 0.136 & 55.000 & 65.787 & 0.0143 & 0.0005 & 0.0051 \\
\hline 20 & 7 & 0.008 & 4.287 & 0.154 & 55.000 & 63.848 & 0.0160 & 0.0006 & 0.0058 \\
\hline 21 & 15 & 0.015 & 4.300 & 0.136 & 55.000 & 67.883 & 0.0227 & 0.0008 & 0.0058 \\
\hline
\end{tabular}

ROCK-DEPENDENT RESULTS FOR PENETRATION RATE $=10.00 \mathrm{FT} / \mathrm{HR}$

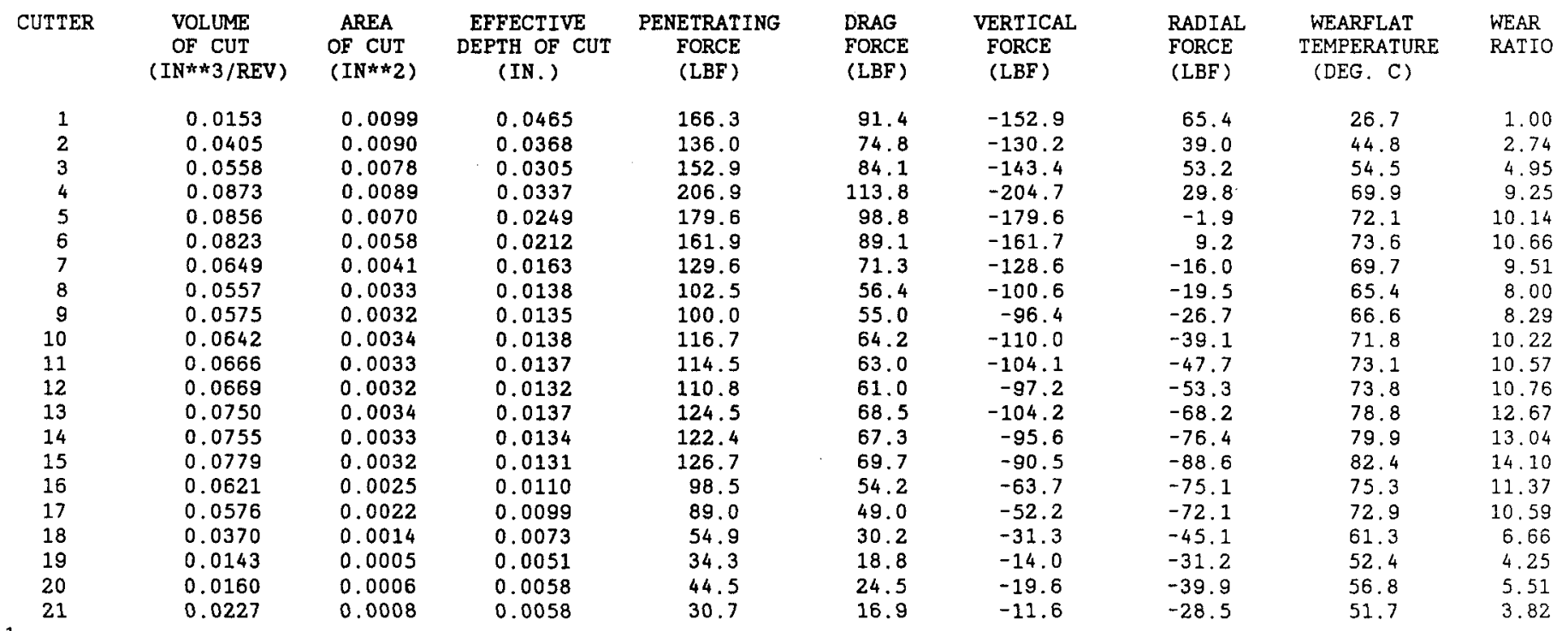

INTEGRATED FORCES AND MOMENTS FOR THE FULL BIT:

$\begin{array}{llr}\text { SIERRA WHITE GRANITE AT ROP } & & 10.0 \\ \text { TOTAL WOB (LBF) } & = & 2092.0 \\ \text { DRILLING TORQ. (FT-LBF) } & = & 282.4 \\ \text { X SIDE FORCE (LBF) } & = & -7.6 \\ \text { Y SIDE FORCE (LBF) } & = & -97.1 \\ \text { RESULTANT SIDE FORCE (LBF) } & = & 97.4 \\ \text { B. M. ABOUT X-AXIS (FT-LBF) } & =9.6 \\ \text { B. M. ABOUT Y-AXIS (FT-LBF) } & =-1.3\end{array}$


GEOMETRY-DEPENDENT RESULTS FOR PENETRATION RATE $=20.00 \mathrm{FT} / \mathrm{HR}$

\begin{tabular}{|c|c|c|c|c|c|c|c|c|c|}
\hline CUTTER & $\begin{array}{l}\text { CUTT ING } \\
\text { ORDER }\end{array}$ & $\begin{array}{r}\text { CUTTING } \\
\text { HEIGHT } \\
\text { (IN.) }\end{array}$ & $\begin{array}{l}\text { WEARFLAT } \\
\text { LOCATION } \\
\text { XBC } \\
\text { (IN.) }\end{array}$ & $\begin{array}{l}\text { WEARFLAT } \\
\text { LOCATION } \\
\text { ZBC } \\
\text { (IN.) }\end{array}$ & $\begin{array}{l}\text { CUTTER } \\
\text { INCL. } \\
\text { ANGLE } \\
\text { (DEG.) }\end{array}$ & $\begin{array}{l}\text { CUTTER } \\
\text { WEAR } \\
\text { ANGLE } \\
\text { (DEG.) }\end{array}$ & $\begin{array}{l}\text { VOLUME } \\
\text { OF } \\
\text { CUT } \\
\text { (IN* } 3 \text { / } \\
\text { REV ) }\end{array}$ & $\begin{array}{l}\text { AREA } \\
\text { OF } \\
\text { CUT } \\
\left(I N^{*} 2\right)\end{array}$ & $\begin{array}{c}\text { EFFECTIVE } \\
\text { DEPTH } \\
\text { OF CUT } \\
\text { (IN.) }\end{array}$ \\
\hline 1 & 20 & 0.665 & 0.212 & 0.982 & -15.000 & -22.557 & 0.0309 & 0.0198 & 0.0652 \\
\hline 2 & 8 & 0.776 & 0.698 & 1.103 & -10.000 & -16.561 & 0.0814 & 0.0180 & 0.0560 \\
\hline 3 & 14 & 0.923 & 1.124 & 1.241 & -5.000 & -19.724 & 0.1129 & 0.0157 & 0.0492 \\
\hline 4 & 18 & 1.035 & 1.552 & 1.370 & -2.000 & -7.433 & 0.1741 & 0.0178 & 0.0530 \\
\hline 5 & 4 & 1.008 & 1.958 & 1.346 & 0.000 & 1.249 & 0.1714 & 0.0139 & 0.0433 \\
\hline 6 & 11 & 1.022 & 2.288 & 1.360 & 2.000 & -1.504 & 0.1638 & 0.0115 & 0.0375 \\
\hline 7 & 21 & 1.030 & 2.542 & 1.366 & 5.000 & 7.669 & 0.1357 & 0.0085 & 0.0297 \\
\hline 8 & 6 & 0.983 & 2.707 & 1. 317 & 10.000 & 11.930 & 0.1105 & 0.0065 & 0.0244 \\
\hline 9 & 13 & 0.967 & 2.872 & 1.294 & 15.000 & 16.273 & 0.1092 & 0.0061 & 0.0231 \\
\hline 10 & 2 & 0.902 & 3.033 & 1.221 & 20.000 & 20.263 & 0.1335 & 0.0070 & 0.0255 \\
\hline 11 & 9 & 0.862 & 3.201 & 1.169 & 25.000 & 25.397 & 0.1320 & 0.0066 & 0.0242 \\
\hline 12 & 16 & 0.807 & 3.362 & 1.103 & 30.000 & 29.543 & 0.1292 & 0.0061 & 0.0229 \\
\hline 13 & 3 & 0.702 & 3.524 & 0.984 & 35.000 & 33.979 & 0.1527 & 0.0069 & 0.0249 \\
\hline 14 & 10 & 0.615 & 3.688 & 0.879 & 40.000 & 39.064 & 0.1500 & 0.0065 & 0.0238 \\
\hline 15 & 17 & 0.507 & 3.858 & 0.744 & 45.000 & 45.617 & 0.1604 & 0.0066 & 0.0240 \\
\hline 16 & 19 & 0.385 & 4.002 & 0.597 & 47.500 & 51.050 & 0.1189 & 0.0047 & 0.0189 \\
\hline 17 & 5 & 0.233 & 4.112 & 0.432 & 50.000 & 53.758 & 0.1153 & 0.0045 & 0.0180 \\
\hline 18 & 12 & 0.128 & 4.199 & 0.321 & 52.500 & 55.450 & 0.0617 & 0.0023 & 0.0111 \\
\hline 19 & 1 & 0.003 & 4.287 & 0.151 & 55.000 & 63.475 & 0.0374 & 0.0014 & 0.0089 \\
\hline 20 & 7 & 0.016 & 4.280 & 0.177 & 55.000 & 61.325 & 0.0452 & 0.0017 & 0.0105 \\
\hline 21 & 15 & 0.029 & 4.298 & 0.156 & 55.000 & 67.023 & 0.0354 & 0.0013 & 0.0077 \\
\hline
\end{tabular}

ROCK-DEPENDENT RESULTS FOR PENETRATION RATE $=20.00 \mathrm{FT} / \mathrm{HR}$

\begin{tabular}{|c|c|c|c|c|c|c|c|c|c|}
\hline CUTTER & $\begin{array}{c}\text { VOLUME } \\
\text { OF CUT } \\
(I N * 3 / R E V)\end{array}$ & $\begin{array}{l}\text { AREA } \\
\text { OF CUT } \\
\left(I N^{\star \star 2} 2\right)\end{array}$ & $\begin{array}{l}\text { EFFECTIVE } \\
\text { DEPTH OF CUT } \\
\text { (IN.) }\end{array}$ & $\begin{array}{l}\text { PENETRAT ING } \\
\text { FORCE } \\
\text { (IBE) }\end{array}$ & $\begin{array}{l}\text { DRAG } \\
\text { FORCE } \\
\text { (LBE) }\end{array}$ & $\begin{array}{l}\text { VERTICAL } \\
\text { FORCE } \\
\text { (LBF) }\end{array}$ & $\begin{array}{l}\text { RADIAL } \\
\text { FORCE } \\
\text { (LBE) }\end{array}$ & $\begin{array}{l}\text { WEARFLAT } \\
\text { TEMPERATURE } \\
\text { (DEG. C) }\end{array}$ & $\begin{array}{l}\text { WEAR } \\
\text { RATIO }\end{array}$ \\
\hline 1 & 0.0309 & 0.0198 & 0.0652 & 289.6 & 159.3 & -267.4 & 111.1 & 26.7 & 1.00 \\
\hline 2 & 0.0814 & 0.0180 & 0.0560 & 225.6 & 124.1 & -216.2 & 64.3 & 26.7 & 2.57 \\
\hline 3 & 0.1129 & 0.0157 & 0.0492 & 186.9 & 102.8 & -175.9 & 63.1 & 60.8 & 3.43 \\
\hline 4 & 0.1741 & 0.0178 & 0.0530 & 250.2 & 137.6 & -248.1 & 32.4 & 79.1 & 6.33 \\
\hline 5 & 0.1714 & 0.0139 & 0.0433 & 226.6 & 124.6 & -226.5 & -4.9 & 84.1 & 7.23 \\
\hline 6 & 0.1638 & 0.0115 & 0.0375 & 205.6 & 113.1 & -205.5 & 5.4 & 86.5 & 7.67 \\
\hline 7 & 0.1357 & 0.0085 & 0.0297 & 166.9 & 91.8 & -165.4 & -22.3 & 82.2 & 6.92 \\
\hline 8 & 0.1105 & 0.0065 & 0.0244 & 130.1 & 71.5 & -127.2 & -26.9 & 75.9 & 5.74 \\
\hline 9 & 0.1092 & 0.0061 & 0.0231 & 125.1 & 68.8 & -120.1 & -35.1 & 76.6 & 5.86 \\
\hline 10 & 0.1335 & 0.0070 & 0.0255 & 150.8 & 83.0 & -141.5 & -52.2 & 85.1 & 7.46 \\
\hline 11 & 0.1320 & 0.0066 & 0.0242 & 145.5 & 80.0 & -131.4 & -62.4 & 85.8 & 7.59 \\
\hline 12 & 0.1292 & 0.0061 & 0.0229 & 139.8 & 76.9 & -121.6 & -68.9 & 86.1 & 7.66 \\
\hline 13 & 0.1527 & 0.0069 & 0.0249 & 160.0 & 88.0 & -132.6 & -89.4 & 93.7 & 9.19 \\
\hline 14 & 0.1500 & 0.0065 & 0.0238 & 155.6 & 85.6 & -120.9 & -98.1 & 94.4 & 9.36 \\
\hline 15 & 0.1604 & 0.0066 & 0.0240 & 163.3 & 89.8 & -114.2 & -116.7 & 98.6 & 10.27 \\
\hline 16 & 0.1189 & 0.0047 & 0.0189 & 123.6 & 68.0 & -77.7 & -96.2 & 87.8 & 8.07 \\
\hline 17 & 0.1153 & 0.0045 & 0.0180 & 114.4 & 62.9 & -67.6 & -92.2 & 86.1 & 7.67 \\
\hline 18 & 0.0617 & 0.0023 & 0.0111 & 65.3 & 35.9 & -37.1 & -53.8 & 67.9 & 4.47 \\
\hline 19 & 0.0374 & 0.0014 & 0.0089 & 43.5 & 23.9 & -19.4 & -38.9 & 59.3 & 3.04 \\
\hline 20 & 0.0452 & 0.0017 & 0.0105 & 56.9 & 31.3 & -27.3 & -49.9 & 65.2 & 3.97 \\
\hline 21 & 0.0354 & 0.0013 & 0.0077 & 34.8 & 19.1 & -13.6 & -32.0 & 55.0 & 2.44 \\
\hline
\end{tabular}

INTEGRATED FORCES AND MOMENTS FOR THE FULL BIT:

$\begin{array}{llr}\text { SIERRA WHITE GRANITE AT ROP } & 20.0 \\ \text { TOTAL WOB (LBF) } & =2757.4 \\ \text { DRILLING TORQ. (FT-LBF) } & =359.6 \\ \text { X SIDE FORCE (LBF) } & =34.2 \\ \text { Y SIDE FORCE (LBF) } & =135.1 \\ \text { RESULTANT SIDE FORCE (LBF) } & =139.3 \\ \text { B. M. ABOUT X-AXIS (FT-LBF) } & 5.4 \\ \text { B. M. ABOUT Y-AXIS (FT-LBF) } & \end{array}$


GEOMETRY-DEPENDENT RESULIS FOR PENETRATION RATE $=30.00 \mathrm{FT} / \mathrm{HR}$

\begin{tabular}{|c|c|c|c|c|c|c|c|c|c|}
\hline CUTTER & $\begin{array}{c}\text { CUTTING } \\
\text { ORDER }\end{array}$ & $\begin{array}{r}\text { CUTTING } \\
\text { HEIGHT } \\
\text { (IN.) }\end{array}$ & $\begin{array}{l}\text { WEARFLAT } \\
\text { LOCATION } \\
\text { XBC } \\
\text { (IN.) }\end{array}$ & $\begin{array}{c}\text { WEARFLAT } \\
\text { LOCATION } \\
\text { ZBC } \\
\text { (IN.) }\end{array}$ & $\begin{array}{l}\text { CUTTER } \\
\text { INCL. } \\
\text { ANGLE } \\
\text { (DEG.) }\end{array}$ & $\begin{array}{l}\text { CUTTER } \\
\text { WEAR } \\
\text { ANGLE } \\
\text { (DEG.) }\end{array}$ & $\begin{array}{l}\text { VOLUME } \\
\text { OF } \\
\text { CUT } \\
\text { (IN**3/ } \\
\text { REV ) }\end{array}$ & $\begin{array}{l}\text { AREA } \\
\text { OF } \\
\text { CUT } \\
(I N * * 2)\end{array}$ & $\begin{array}{c}\text { EFFECTIVE } \\
\text { DEPTH } \\
\text { OF CUT } \\
\text { (IN.) }\end{array}$ \\
\hline 1 & 20 & 0.685 & 0.216 & 1.004 & -15.000 & -21.959 & 0.0465 & 0.0298 & 0.0836 \\
\hline 2 & 8 & 0.784 & 0.700 & 1.111 & -10.000 & -16.169 & 0.1228 & 0.0271 & 0.0745 \\
\hline 3 & 14 & 0.937 & 1.128 & 1.256 & -5.000 & -19.090 & 0.1714 & 0.0238 & 0.0669 \\
\hline 4 & 18 & 1.053 & 1.558 & 1.388 & -2.000 & -6.463 & 0.2605 & 0.0265 & 0.0709 \\
\hline 5 & 4 & 1.013 & 1.961 & 1.351 & 0.000 & 1.593 & 0.2575 & 0.0209 & 0.0602 \\
\hline 6 & 11 & 1.033 & 2.301 & 1.371 & 2.000 & 0.331 & 0.2446 & 0.0171 & 0.0514 \\
\hline 7 & 21 & 1.050 & 2.530 & 1.388 & 5.000 & 5.854 & 0.2112 & 0.0133 & 0.0423 \\
\hline 8 & 6 & 0.990 & 2.702 & 1.324 & 10.000 & 11.213 & 0.1623 & 0.0095 & 0.0330 \\
\hline 9 & 13 & 0.980 & 2.870 & 1.308 & 15.000 & 15.989 & 0.1603 & 0.0089 & 0.0313 \\
\hline 10 & 2 & 0.905 & 3.035 & 1.223 & 20.000 & 20.638 & 0.2051 & 0.0108 & 0.0359 \\
\hline 11 & 9 & 0.871 & 3.204 & 1.177 & 25.000 & 25.797 & 0.1967 & 0.0098 & 0.0333 \\
\hline 12 & 16 & 0.823 & 3.365 & 1.117 & 30.000 & 30.073 & 0.1890 & 0.0090 & 0.0311 \\
\hline 13 & 3 & 0.706 & 3.525 & 0.987 & 35.000 & 34.235 & 0.2308 & 0.0104 & 0.0348 \\
\hline 14 & 10 & 0.626 & 3.692 & 0.886 & 40.000 & 39.797 & 0.2228 & 0.0096 & 0.0327 \\
\hline 15 & 17 & 0.525 & 3.862 & 0.757 & 45.000 & 46.464 & 0.2465 & 0.0102 & 0.0340 \\
\hline 16 & 19 & 0.404 & 4.008 & 0.607 & 47.500 & 52.640 & 0.1703 & 0.0068 & 0.0253 \\
\hline 17 & 5 & 0.239 & 4.113 & 0.436 & 50.000 & 54.053 & 0.1728 & 0.0067 & 0.0251 \\
\hline 18 & 12 & 0.141 & 4.200 & 0.332 & 52.500 & 55.718 & 0.0821 & 0.0031 & 0.0141 \\
\hline 19 & 1 & 0.004 & 4.283 & 0.161 & 55.000 & 62.026 & 0.0642 & 0.0024 & 0.0130 \\
\hline 20 & 7 & 0.024 & 4.280 & 0.185 & 55.000 & 61.325 & 0.0743 & 0.0028 & 0.0140 \\
\hline 21 & 15 & 0.044 & 4.297 & 0.174 & 55.000 & 66.497 & 0.0504 & 0.0019 & 0.0104 \\
\hline
\end{tabular}

ROCK-DEPENDENT RESULTS FOR PENETRATION RATE $=30.00 \mathrm{FT} / \mathrm{HR}$

\begin{tabular}{|c|c|c|c|c|c|c|c|c|c|}
\hline CUTTER & $\begin{array}{c}\text { VOLUME } \\
\text { OF CUT } \\
\left(I N^{* * 3 / R E V)}\right.\end{array}$ & $\begin{array}{l}\text { AREA } \\
\text { OF CUT } \\
\text { (IN**2) }\end{array}$ & $\begin{array}{l}\text { EFFECTIVE } \\
\text { DEPTH OF CUT } \\
\text { (IN.) }\end{array}$ & $\begin{array}{l}\text { PENETRATING } \\
\text { FORCE } \\
\text { (LBF) }\end{array}$ & $\begin{array}{l}\text { DRAG } \\
\text { FORCE } \\
\text { (LBF) }\end{array}$ & $\begin{array}{l}\text { VERTICAL } \\
\text { FORCE } \\
\text { (LBF) }\end{array}$ & $\begin{array}{l}\text { RADIAL } \\
\text { FORCE } \\
\text { (LBF) }\end{array}$ & $\begin{array}{l}\text { WEARFLAT } \\
\text { TEMPERATURE } \\
\text { (DEG. C) }\end{array}$ & $\begin{array}{l}\text { WEAR } \\
\text { RATIO }\end{array}$ \\
\hline 1 & 0.0465 & 0.0298 & 0.0836 & 435.7 & 239.7 & -404.1 & 162.9 & 26.7 & 1.00 \\
\hline 4 & 0.2605 & 0.0265 & 0.0709 & 332.6 & 182.9 & -330.4 & 37.4 & 26.7 & 5.52 \\
\hline 5 & 0.2575 & 0.0209 & 0.0602 & 260.2 & 143.1 & -260.1 & -7.2 & 92.6 & 5.43 \\
\hline 6 & 0.2446 & 0.0171 & 0.0514 & 234.7 & 129.1 & -234.7 & -1.4 & 95.2 & 5.75 \\
\hline 7 & 0.2112 & 0.0133 & 0.0423 & 193.5 & 106.4 & -192.5 & -19.7 & 90.8 & 5.21 \\
\hline 10 & 0.2051 & 0.0108 & 0.0359 & 174.3 & 95.8 & -163.1 & -61.4 & 94.2 & 5.63 \\
\hline 11 & 0.1967 & 0.0098 & 0.0333 & 166.4 & 91.5 & -149.8 & -72.4 & 94.3 & 5.68 \\
\hline 12 & 0.1890 & 0.0090 & 0.0311 & 158.9 & 87.4 & -137.6 & -79.6 & 94.3 & 5.69 \\
\hline 13 & 0.2308 & 0.0104 & 0.0348 & 184.0 & 101.2 & -152.1 & -103.5 & 103.8 & 6.90 \\
\hline 14 & 0.2228 & 0.0096 & 0.0327 & 177.9 & 97.9 & -136.7 & -113.9 & 104.2 & 6.99 \\
\hline 15 & 0.2465 & 0.0102 & 0.0340 & 189.0 & 103.9 & -130.2 & -137.0 & 109.9 & 7.77 \\
\hline 16 & 0.1703 & 0.0068 & 0.0253 & 139.8 & 76.9 & -84.8 & -111.1 & 95.9 & 5.97 \\
\hline
\end{tabular}

INTEGRATED FORCES AND MOMENTS FOR THE FULL BIT:

$\begin{array}{llr}\text { SIERRA WHITE GRANITE AT ROP } & 30.0 \\ \text { TOTAL WOB (LBF) } & =3477.8 \\ \text { DRILLING IORQ. (FT-LBF) } & =424.0 \\ \text { X SIDE FORCE (LBF) } & =22.3 \\ \text { Y SIDE FORCE (LBF) } & = & -181.7 \\ \text { RESULTANT SIDE FORCE (LBF) } & =183.0 \\ \text { B. M. ABOUT X-AXIS (FT-LBF) } & 14.9 \\ \text { B. M. ABOUT Y-AXIS (FT-LBF) } & -1.5\end{array}$


GEOMETRY-DEPENDENT RESULTS FOR PENETRATION RATE $=40.00 \mathrm{FT} / \mathrm{HR}$

\begin{tabular}{|c|c|c|c|c|c|c|c|c|c|}
\hline CUTTER & $\begin{array}{l}\text { CUTTING } \\
\text { ORDER }\end{array}$ & $\begin{array}{r}\text { CUTTING } \\
\text { HEIGHT } \\
\text { (IN.) }\end{array}$ & $\begin{array}{l}\text { WEARFLAT } \\
\text { LOCATION } \\
\text { XBC } \\
\text { (IN.) }\end{array}$ & $\begin{array}{l}\text { WEARFLAT } \\
\text { LOCATION } \\
\text { ZBC } \\
\text { (IN.) }\end{array}$ & $\begin{array}{l}\text { CUTTER } \\
\text { INCL . } \\
\text { ANGLE } \\
\text { (DEG .) }\end{array}$ & $\begin{array}{l}\text { CUTTER } \\
\text { WEAR } \\
\text { ANGLE } \\
\text { (DEG.) }\end{array}$ & $\begin{array}{l}\text { VOLUME } \\
\text { OF } \\
\text { CUT } \\
\text { (IN**3/ } \\
\text { REV) }\end{array}$ & $\begin{array}{l}\text { AREA } \\
\text { OF } \\
\text { CUT } \\
(I N * 2)\end{array}$ & $\begin{array}{l}\text { EFFECTIVE } \\
\text { DEPTH } \\
\text { OF CUT } \\
\text { (IN.) }\end{array}$ \\
\hline 1 & 20 & 0.705 & 0.219 & 1.025 & -15.000 & -21.355 & 0.0624 & 0.0399 & 0.1018 \\
\hline 2 & 8 & 0.792 & 0.701 & 1.120 & -10.000 & -16.084 & 0.1646 & 0.0362 & 0.0923 \\
\hline 3 & 14 & 0.951 & 1.132 & 1.271 & -5.000 & -18.456 & 0.2314 & 0.0320 & 0.0838 \\
\hline 4 & 18 & 1.070 & 1.565 & 1.406 & -2.000 & -5.535 & 0.3462 & 0.0351 & 0.0879 \\
\hline 5 & 4 & 1.017 & 1.965 & 1.355 & 0.000 & 2.288 & 0.3440 & 0.0279 & 0.0756 \\
\hline 6 & 11 & 1.043 & 2.305 & 1.382 & 2.000 & 0.978 & 0.3243 & 0.0226 & 0.0641 \\
\hline 7 & 21 & 1.070 & 2.526 & 1.408 & 5.000 & 5.174 & 0.2814 & 0.0177 & 0.0529 \\
\hline 8 & 6 & 0.997 & 2.697 & 1.332 & 10.000 & 10.423 & 0.2143 & 0.0126 & 0.0412 \\
\hline 9 & 13 & 0.993 & 2.864 & 1.323 & 15.000 & 15.108 & 0.2145 & 0.0119 & 0.0394 \\
\hline 10 & 2 & 0.907 & 3.027 & 1.228 & 20.000 & 19.397 & 0.2804 & 0.0147 & 0.0458 \\
\hline 11 & 9 & 0.880 & 3.200 & 1.187 & 25.000 & 25.169 & 0.2588 & 0.0128 & 0.0413 \\
\hline 12 & 16 & 0.838 & 3. 368 & 1. 131 & 30.000 & 30.550 & 0.2490 & 0.0118 & 0.0387 \\
\hline 13 & 3 & 0.710 & 3.528 & 0.989 & 35.000 & 34.702 & 0.3096 & 0.0140 & 0.0438 \\
\hline 14 & 10 & 0.636 & 3.696 & 0.894 & 40.000 & 40.488 & 0.2956 & 0.0127 & 0.0408 \\
\hline 15 & 17 & 0.542 & 3. 864 & 0.772 & 45.000 & 47.062 & 0.3356 & 0.0139 & 0.0433 \\
\hline 16 & 19 & 0.423 & 4.012 & 0.621 & 47.500 & 53.500 & 0.2191 & 0.0087 & 0.0308 \\
\hline 17 & 5 & 0.244 & 4.115 & 0.439 & 50.000 & 54.588 & 0.2267 & 0.0088 & 0.0312 \\
\hline 18 & 12 & 0.153 & 4. 201 & 0.344 & 52.500 & 55.796 & 0.1049 & 0.0040 & 0.0172 \\
\hline 19 & 1 & 0.006 & 4.278 & 0.172 & 55.000 & 60.467 & 0.0938 & 0.0035 & 0.0170 \\
\hline 20 & 7 & 0.032 & 4.280 & 0.193 & 55.000 & 61.325 & 0.1004 & 0.0038 & 0.0171 \\
\hline 21 & 15 & 0.059 & 4.296 & 0.191 & 55.000 & 66.109 & 0.0657 & 0.0024 & 0.0126 \\
\hline
\end{tabular}

ROCK-DEPENDENT RESULTS FOR PENETRATION RATE $=40.00 \mathrm{FT} / \mathrm{HR}$

\begin{tabular}{|c|c|c|c|c|c|c|c|c|c|}
\hline CUTTER & $\begin{array}{c}\text { VOLUME } \\
\text { OF CUT } \\
\text { (IN**3/REV) }\end{array}$ & $\begin{array}{l}\text { AREA } \\
\text { OF CUT } \\
(I N \star \star 2)\end{array}$ & $\begin{array}{l}\text { EFFECTIVE } \\
\text { DEPTH OF CUT } \\
\text { (IN.) }\end{array}$ & $\begin{array}{c}\text { PENETRATING } \\
\text { FORCE } \\
\text { (LBF) }\end{array}$ & $\begin{array}{l}\text { DRAG } \\
\text { FORCE } \\
\text { (LBF) }\end{array}$ & $\begin{array}{l}\text { VERIICAL } \\
\text { FORCE } \\
\text { (LBF) }\end{array}$ & $\begin{array}{l}\text { RADIAL } \\
\text { FORCE } \\
\text { (LBF) }\end{array}$ & $\begin{array}{l}\text { WEARFLAT } \\
\text { IEMPERATURE } \\
\text { (DEG. C) }\end{array}$ & $\begin{array}{l}\text { WEAR } \\
\text { RATIO }\end{array}$ \\
\hline 1 & 0.0624 & 0.0399 & 0.1018 & 601.8 & 331.0 & -560.5 & 219.1 & 26.7 & 1.00 \\
\hline 2 & 0.1646 & 0.0362 & 0.0923 & 512.6 & 281.9 & -492.5 & 142.0 & 26.7 & 2.72 \\
\hline 3 & 0.2314 & 0.0320 & 0.0838 & 437.3 & 240.5 & -414.8 & 138.5 & 26.7 & 3.75 \\
\hline 4 & 0.3462 & 0.0351 & 0.0879 & 472.5 & 259.9 & -470.3 & 45.6 & 26.7 & 5.60 \\
\hline 5 & 0.3440 & 0.0279 & 0.0756 & 369.4 & 203.2 & -369.1 & -14.7 & 26.7 & 5.50 \\
\hline 6 & 0.3243 & 0.0226 & 0.0641 & 281.8 & 155.0 & -281.7 & -4.8 & 26.7 & 4.92 \\
\hline 7 & 0.2814 & 0.0177 & 0.0529 & 212.6 & 117.0 & -211.8 & -19.2 & 97.1 & 4.07 \\
\hline 8 & 0.2143 & 0.0126 & 0.0412 & 162.1 & 89.2 & -159.5 & -29.3 & 87.9 & 3.31 \\
\hline 9 & 0.2145 & 0.0119 & 0.0394 & 156.7 & 86.2 & -151.3 & -40.8 & 89.1 & 3.40 \\
\hline 10 & 0.2804 & 0.0147 & 0.0458 & 193.1 & 106.2 & -182.1 & -64.1 & 101.4 & 4.43 \\
\hline 11 & 0.2588 & 0.0128 & 0.0413 & 182.2 & 100.2 & -164.9 & -77.5 & 100.7 & 4.42 \\
\hline 12 & 0.2490 & 0.0118 & 0.0387 & 174.1 & 95.8 & -150.0 & -88.5 & 100.9 & 4.44 \\
\hline 13 & 0.3096 & 0.0140 & 0.0438 & 202.7 & 111.5 & -166.6 & -115.4 & 111.7 & 5.42 \\
\hline 14 & 0.2956 & 0.0127 & 0.0408 & 195.3 & 107.4 & -148.6 & -126.8 & 111.8 & 5.47 \\
\hline 15 & 0.3356 & 0.0139 & 0.0433 & 209.2 & 115.1 & -142.5 & -153.2 & 118.9 & 6.12 \\
\hline 16 & 0.2191 & 0.0087 & 0.0308 & 152.0 & 83.6 & -90.4 & -122.2 & 102.0 & 4.62 \\
\hline 17 & 0.2267 & 0.0088 & 0.0312 & 144.1 & 79.3 & -83.5 & -117.5 & 101.6 & 4.49 \\
\hline 18 & 0.1049 & 0.0040 & 0.0172 & 78.6 & 43.2 & -44.2 & -65.0 & 76.3 & 2.50 \\
\hline 19 & 0.0938 & 0.0035 & 0.0170 & 57.0 & 31.4 & -28.1 & -49.6 & 69.4 & 1.85 \\
\hline 20 & 0.1004 & 0.0038 & 0.0171 & 69.9 & 38.4 & -33.5 & -61.3 & 74.0 & 2.27 \\
\hline 21 & 0.0657 & 0.0024 & 0.0126 & 42.7 & 23.5 & -17.3 & -39.0 & 61.4 & 1.39 \\
\hline
\end{tabular}

1

INTEGRATED FORCES AND MOMENTS FOR THE FULL BIT:

SIERRA WHITE GRANITE AT ROP $=40.0$

TOTAL WOB (LBF)

$=4363.2$

DRILLING TORQ. (FT-LBF)

$=\quad 494.2$

$X$ SIDE FORCE (LBF)

$=\quad 14.2$

Y SIDE FORCE (LBF)

$=-253.3$

RESULTANT SIDE FORCE (LBF) $=253.7$

B. M. ABOUT X-AXIS $($ FT-LBF $)=24.0$

B. M. ABOUT Y-AXIS (FT-LBF) $=-0.1$ 


\begin{tabular}{|c|c|c|c|c|c|c|c|c|c|}
\hline CUTTER & $\begin{array}{l}\text { CUTTING } \\
\text { ORDER }\end{array}$ & $\begin{array}{c}\text { CUTTING } \\
\text { HEIGHT } \\
\text { (IN.) }\end{array}$ & $\begin{array}{l}\text { WEARFLAT } \\
\text { LOCATION } \\
\text { XBC } \\
\text { (IN.) }\end{array}$ & $\begin{array}{c}\text { WEARFLAT } \\
\text { LOCATION } \\
\text { ZBC } \\
\text { (IN.) }\end{array}$ & $\begin{array}{l}\text { CUTTER } \\
\text { INCL. } \\
\text { ANGLE } \\
\text { (DEG.) }\end{array}$ & $\begin{array}{l}\text { CUTTER } \\
\text { WEAR } \\
\text { ANGLE } \\
\text { (DEG.) }\end{array}$ & $\begin{array}{l}\text { VOLUME } \\
\text { OF } \\
\text { CUT } \\
\text { (IN**3/ } \\
\text { REV) }\end{array}$ & $\begin{array}{c}\text { AREA } \\
\text { OF } \\
\text { CUT } \\
(I N * * 2)\end{array}$ & $\begin{array}{c}\text { EFFECTIVE } \\
\text { DEPTH } \\
\text { OF CUT } \\
\text { (IN.) }\end{array}$ \\
\hline 1 & 20 & 0.725 & 0.225 & 1.047 & -15.000 & -20.436 & 0.0785 & 0.0500 & 0.1198 \\
\hline 2 & 8 & 0.800 & 0.703 & 1.128 & -10.000 & -15.700 & 0.2070 & 0.0454 & 0.1095 \\
\hline 3 & 14 & 0.964 & 1.140 & 1. 288 & -5.000 & -17.283 & 0.2928 & 0.0403 & 0.1003 \\
\hline 4 & 18 & 1.087 & 1.569 & 1.424 & -2.000 & -4.914 & 0.4311 & 0.0436 & 0.1038 \\
\hline 5 & 4 & 1.021 & 1.970 & 1.359 & 0.000 & 2.998 & 0.4307 & 0.0349 & 0.0904 \\
\hline 6 & 11 & 1.054 & 2.305 & 1.393 & 2,000 & 0.984 & 0.4004 & 0.0279 & 0.0762 \\
\hline 7 & 21 & 1.090 & 2.520 & 1.429 & 5.000 & 4.313 & 0.3506 & 0.0220 & 0.0627 \\
\hline 8 & 6 & 1.003 & 2.694 & 1.339 & 10.000 & 9.974 & 0.2637 & 0.0155 & 0.0484 \\
\hline 9 & 13 & 1.007 & 2.861 & 1.337 & 15.000 & 14.600 & 0.2675 & 0.0148 & 0.0468 \\
\hline 10 & 2 & 0.909 & 3.026 & 1.230 & 20.000 & 19.149 & 0.3576 & 0.0188 & 0.0554 \\
\hline 11 & 9 & 0.888 & 3.196 & 1. 198 & 25.000 & 24.547 & 0.3229 & 0.0160 & 0.0491 \\
\hline 12 & 16 & 0.854 & 3.365 & 1.148 & 30.000 & 30.014 & 0.3127 & 0.0148 & 0.0462 \\
\hline 13 & 3 & 0.713 & 3.530 & 0.991 & 35.000 & 35.002 & 0.3898 & 0.0176 & 0.0524 \\
\hline 14 & 10 & 0.647 & 3.696 & 0.904 & 40.000 & 40.622 & 0.3679 & 0.0159 & 0.0484 \\
\hline 15 & 17 & 0.559 & 3.867 & 0.786 & 45.000 & 47.573 & 0.4252 & 0.0175 & 0.0521 \\
\hline 16 & 19 & 0.441 & 4.016 & 0.634 & 47.500 & 54.658 & 0.2677 & 0.0106 & 0.0360 \\
\hline 17 & 5 & 0.250 & 4.112 & 0.449 & 50.000 & 53.811 & 0.2780 & 0.0108 & 0.0369 \\
\hline 18 & 12 & 0.165 & 4.196 & 0.362 & 52.500 & 54.653 & 0.1271 & 0.0048 & 0.0201 \\
\hline 19 & 1 & 0.007 & 4.273 & 0.181 & 55.000 & 59.131 & 0.1265 & 0.0047 & 0.0212 \\
\hline 20 & 7 & 0.040 & 4.280 & 0.201 & 55.000 & 61.325 & 0.1263 & 0.0047 & 0.0202 \\
\hline 21 & 15 & 0.074 & 4.295 & 0.207 & 55.000 & 65.912 & 0.0795 & 0.0029 & 0.0146 \\
\hline
\end{tabular}

ROCK-DEPENDENT RESULTS FOR PENETRATION RATE $=50.00 \mathrm{FT} / \mathrm{HR}$

\begin{tabular}{|c|c|c|c|c|c|c|c|c|c|}
\hline CUTTER & $\begin{array}{c}\text { VOLUME } \\
\text { OF CUT } \\
\left(I N^{* * 3 / R E V)}\right.\end{array}$ & $\begin{array}{l}\text { AREA } \\
\text { OF CUT } \\
\text { (IN**2) }\end{array}$ & $\begin{array}{l}\text { EFFECTIVE } \\
\text { DEPTH OF CUT } \\
\text { (IN.) }\end{array}$ & $\begin{array}{c}\text { PENETRATING } \\
\text { FORCE } \\
\text { (LBF) }\end{array}$ & $\begin{array}{l}\text { DRAG } \\
\text { FORCE } \\
\text { (LBF) }\end{array}$ & $\begin{array}{l}\text { VERTICAL } \\
\text { FORCE } \\
\text { (LBF) }\end{array}$ & $\begin{array}{l}\text { RADIAL } \\
\text { FORCE } \\
\text { (LBF) }\end{array}$ & $\begin{array}{l}\text { WEARFLAT } \\
\text { TEMPERATURE } \\
\text { (DEG. C) }\end{array}$ & $\begin{array}{l}\text { WEAR } \\
\text { RATIO }\end{array}$ \\
\hline 1 & 0.0785 & 0.0500 & 0.1198 & 785.2 & 431.9 & -735.8 & 274.2 & 26.7 & 1.00 \\
\hline 2 & 0.2070 & 0.0454 & 0.1095 & 677.9 & 372.9 & -652.7 & 183.5 & 26.7 & 2.70 \\
\hline 3 & 0.2928 & 0.0403 & 0.1003 & 586.9 & 322.8 & -560.4 & 174.4 & 26.7 & 3.78 \\
\hline 4 & 0.4311 & 0.0436 & 0.1038 & 620.9 & 341.5 & -618.6 & 53.2 & 26.7 & 5.51 \\
\hline 5 & 0.4307 & 0.0349 & 0.0904 & 495.4 & 272.5 & -494.7 & -25.9 & 26.7 & 5.52 \\
\hline 6 & 0.4004 & 0.0279 & 0.0762 & 374.2 & 205.8 & -374.2 & -6.4 & 26.7 & 4.88 \\
\hline 7 & 0.3506 & 0.0220 & 0.0627 & 271.4 & 149.3 & -270.6 & -20.4 & 26.7 & 3.87 \\
\hline 8 & 0.2637 & 0.0155 & 0.0484 & 177.4 & 97.6 & -174.7 & -30.7 & 26.7 & 2.70 \\
\hline 9 & 0.2675 & 0.0148 & 0.0468 . & 168.3 & 92.6 & -162.9 & -42.4 & 93.7 & 2.72 \\
\hline 10 & 0.3576 & 0.0188 & 0.0554 & 221.5 & 121.8 & -209.2 & -72.7 & 26.7 & 3.79 \\
\hline 11 & 0.3229 & 0.0160 & 0.0491 & 195.9 & 107.8 & -178.2 & -81.4 & 106.2 & 3.54 \\
\hline 12 & 0.3127 & 0.0148 & 0.0462 & 187.6 & 103.2 & -162.4 & -93.8 & 106.5 & 3.57 \\
\hline 13 & 0.3898 & 0.0176 & 0.0524 & 218.5 & 120.2 & -179.0 & -125.3 & 118.4 & 4.36 \\
\hline 14 & 0.3679 & 0.0159 & 0.0484 & 209.7 & 115.4 & -159.2 & -136.6 & 118.1 & 4.38 \\
\hline 15 & 0.4252 & 0.0175 & 0.0521 & 226.1 & 124.3 & -152.5 & -166.9 & 126.4 & 4.94 \\
\hline 16 & 0.2677 & 0.0106 & 0.0360 & 162.3 & 89.3 & -93.9 & -132.4 & 107.1 & 3.69 \\
\hline 17 & 0.2780 & 0.0108 & 0.0369 & 154.7 & 85.1 & -91.3 & -124.8 & 107.0 & 3.60 \\
\hline 18 & 0.1271 & 0.0048 & 0.0201 & 83.9 & 46.1 & -48.5 & -68.4 & 79.6 & 1.99 \\
\hline 19 & 0.1265 & 0.0047 & 0.0212 & 62.5 & 34.4 & -32.1 & -53.7 & 73.5 & 1.51 \\
\hline 20 & 0.1263 & 0.0047 & 0.0202 & 75.0 & 41.2 & -36.0 & -65.8 & 77.5 & 1.81 \\
\hline 21 & 0.0795 & 0.0029 & 0.0146 & 45.4 & 25.0 & -18.5 & -41.4 & 63.6 & 1.10 \\
\hline
\end{tabular}

INTEGRATED FORCES AND MOMENTS FOR THE FULL BIT:

SIERRA WHITE GRANITE AT ROP $=50.0$

TOTAL WOB (LBF) $=5405.5$

DRILLING TORQ. (FT-LBF) $=575.2$

$X$ SIDE FORCE (LBF) = $\quad-3.6$

Y SIDE FORCE (LBF) $\quad=-340.4$

RESULTANT SIDE FORCE (LBF) $=340.4$

B. M. ABOUT X-AXIS $(F T-L B F)=33.4$

B. M. ABOUT Y-AXIS $($ FT-LBF $)=2.1$ 
INTEGRATED AND SUMMARY DATA FOR THE FULL BIT AT ALL SPECIFIED PENETRATION RATES * :

\begin{tabular}{|c|c|c|c|c|c|c|}
\hline SIERRA WHITE GRANITE AT ROP & $=$ & 10.0 & 20.0 & 30.0 & 40.0 & 50.0 \\
\hline $\begin{array}{l}\text { TOTAL WOB (LBF) } \\
\text { DRILLING TORQ. (FT-LBF) }\end{array}$ & $\begin{array}{l}= \\
=\end{array}$ & $\begin{array}{r}2092.0 \\
282.4\end{array}$ & $\begin{array}{r}2757.4 \\
359.6\end{array}$ & $\begin{array}{r}3477.8 \\
424.0\end{array}$ & $\begin{array}{r}4363.2 \\
494.2\end{array}$ & $\begin{array}{r}5405.5 \\
575.2\end{array}$ \\
\hline $\begin{array}{l}X \text { SIDE FORCE (LBF) } \\
Y \text { SIDE FORCE (LBF) } \\
\text { RESULTANT SIDE FORCE (LBF) }\end{array}$ & $\begin{array}{l}= \\
= \\
=\end{array}$ & $\begin{array}{r}-7.6 \\
-97.1 \\
97.4\end{array}$ & $\begin{array}{r}34.2 \\
-135.1 \\
139.3\end{array}$ & $\begin{array}{r}22.3 \\
-181.7 \\
183.0\end{array}$ & $\begin{array}{r}14.2 \\
-253.3 \\
253.7\end{array}$ & $\begin{array}{r}-3.6 \\
-340.4 \\
340.4\end{array}$ \\
\hline $\begin{array}{l}\text { B. M. ABOUT X-AXIS }(F T-L B F)= \\
\text { B. M. ABOUT Y-AXIS }(F T-L B F)=\end{array}$ & $\begin{array}{l}= \\
=\end{array}$ & $\begin{array}{r}9.6 \\
-1.3\end{array}$ & $\begin{array}{l}5.4 \\
1.0\end{array}$ & $\begin{array}{l}14.9 \\
-1.5\end{array}$ & $\begin{array}{l}24.0 \\
-0.1\end{array}$ & $\begin{array}{r}33.4 \\
2.1\end{array}$ \\
\hline $\begin{array}{l}\text { MAX. WEARFLAT TEMP. (DEG. C) } \\
\text { MAX. WEAR RATIO }\end{array}$ & $\begin{aligned}= \\
=\end{aligned}$ & $\begin{array}{r}82.4 \\
14.10\end{array}$ & $\begin{array}{r}98.6 \\
10.27\end{array}$ & $\begin{array}{r}109.9 \\
7.77\end{array}$ & $\begin{array}{r}118.9 \\
6.12\end{array}$ & $\begin{array}{r}126.4 \\
5.52\end{array}$ \\
\hline
\end{tabular}

* BIT DESIGN DATA FROM FILE BITDES.DAT CUTTER WEAR CONFIGURATION FROM FILE WCBO02.DAT

1 OPERATING PARAMETER DATA FROM FILE OPCOND.DAT

****** NEW CUTTER WEAR CONFIGURATION ****

OBTAINED BY SPECIFYING HARD-ROCK WEAR MODE AND NEW WEARFLAT AREA OF 0.0100 FOR CUTTER NUMBER 4

COMPUTED LENGTH OF HOLE SINCE LAST WEAR CONF $=\quad 86.2 \mathrm{FT}$ TOTAL LENGTH OF HOLE DRILLED SINCE START $=147.6 \mathrm{FT}$ (USING ABRASIVE WEAR CONSTANT OF $0.6890 E-12$ )

(THIS CONFIGURATION WAS STORED IN DISK FILE WCB010.DAT )

$\begin{array}{cccc}\text { CUTTER } & \begin{array}{c}\text { WEARFLAT } \\ \text { LENGTH } \\ \text { (IN.) }\end{array} & \begin{array}{c}\text { WEARFLAT } \\ \text { WIDTH } \\ \text { (IN.) }\end{array} & \begin{array}{c}\text { WEARFLAT } \\ \text { AREA } \\ \text { (IN* } * 2)\end{array} \\ 1 & 0.030 & 0.173 & 0.0035 \\ 2 & 0.045 & 0.212 & 0.0064 \\ 3 & 0.051 & 0.225 & 0.0078 \\ 4 & 0.060 & 0.245 & 0.0100 \\ 5 & 0.060 & 0.244 & 0.0099 \\ 6 & 0.060 & 0.244 & 0.0099 \\ 7 & 0.057 & 0.238 & 0.0091 \\ 8 & 0.052 & 0.227 & 0.0080 \\ 9 & 0.052 & 0.227 & 0.0080 \\ 10 & 0.057 & 0.239 & 0.0093 \\ 11 & 0.057 & 0.239 & 0.0092 \\ 12 & 0.057 & 0.238 & 0.0092 \\ 13 & 0.061 & 0.247 & 0.0102 \\ 14 & 0.061 & 0.247 & 0.0103 \\ 15 & 0.064 & 0.252 & 0.0108 \\ 16 & 0.057 & 0.239 & 0.0092 \\ 17 & 0.056 & 0.236 & 0.0089 \\ 18 & 0.044 & 0.210 & 0.0063 \\ 19 & 0.038 & 0.195 & 0.0050 \\ 20 & 0.042 & 0.205 & 0.0058 \\ 21 & 0.034 & 0.185 & 0.0043\end{array}$



DISTRIBUTION

$-189-$ 
DISTRIBUTION:

DOE/TIC-4500-UC-66C (400)

Thomas Ahrens

Seismological Laboratory 252-21

California Institute of Technology

Pasadena, CA 91125

Tom Anderson

Drilling Fluid Consultants

17726 SW Overlook Ln.

Lake Oswego, OR 97034

Chuji Araki

Geothermal Energy Research \& Development Co., LTD

Kyodo Bldg.

11-7, Kabuto-Cho, Nihonbashi

Chuo-Ku, Tokyo. 103, Japan

C. Arkinson

Imperial College of Science \& Technolgy

Dept. of Mathematics

Huxley Bldg.

Queen's Gate, London SW7 2BZ

England

Dr. J . J . Azar

Tulsa University Drilling

Research Projects

Petroleum Engineering Dept.

North Campus Drill Bldg.

2450 Marshal1

Tulsa, OK 74110

Bill Baker

Smith Tool

P.O. Box C-19511

Irvine, CA 92713

Jerry Baird

Jordan, Aposta1, Ritter

Associates, Inc.

Administrative Building 7

Davisville, RI 02854

John Barr

NL Hycalog

15112 Morales Rd.

P.0. Box 60747

Houston, TX 77205
John Barr

NL Hycalog

Oldends Lane

Industrial Estate,

Stonehouse, Glouscester

England GL10 3RQ

Ken Bigelow

Norton Christensen, Inc.

Diamond Technology Center

2532 South 3270 West

Salt Lake City, UT 84119

Ed Bingman

Shell Oil Co.

Two Shell Plaza

P.B. Box 2099

Houston, TX 77001

Alan Black

Drilling Research Laboratory

University Research Park

400 Wakara Way

Salt Lake City, UT 84108

Mike Bockleie

Diamond Technology Center

2532 South 3270 West

Salt Lake City, UT 84119

Gerold R. Boyle

Anco Diamond Composites Corp.

5455 th Ave.

New York, NY 10017

Ben Bradford

Dowe11

P.0. Box 2710

Tulsa, OK 74102

Jim Bresee

U.S. Dept. of Energy

Geothermal Techologies Division

Forrestal Bldg., CE-324

1000 Independence Ave., SW

Washington, DC 20585

Kirk Brownel1

The Robbins Co.

Box 97027

Kent, WA 98031 
John Bunting

US Synthetic Corp.

365 South Mountain Way

Orem UT 84057

Trevor Burgess

Anadrill Schlumberger

200 Macco Blvd.

Sugarland, TX 77478

Dr. Georges Chahine

Tracor Hydronautics, Inc.

7210 Pindel1 School Rd.

Laure1, MD 20810

Curtis Cheatham

Shell Oil Co.

Two Shell Plaza

P.B. Box 2099

Houston, TX 77001

John Cheatham

Rice University

Mechanical Engineering Dept.

P.O. Box 1892

Houston, TX 77001

Weng-Kwen Chia

Smith Tool Co.

P.O. Box C-19511

Irvine, CA 92713

David Clark

Conoco Production Research

P.0. Box 1267

Ponca City, OK 74603

Dr. Andrew F. Conn

Tracor Hydronautics, Inc.

7210 Pindell School Rd.

Laure1, MD 20810

Craig Cooley

Norton Christensen, Inc.

Diamond Technology Center

2532 South 3270 West

Salt Lake City, UT 84119

George Cooper

Schlumberger Cambridge Research

P.O. Box 153

Cambridge CB2 3BE

England
Jim Combs

Geothermal Resources Int'1., Inc.

1825 S. Grant, Suite 900

San Mateo, CA 94402

Brett Davies

Huddy International

Suite 304

7061 S. University Blvd.

Littleton, CO 80122

Ken Davis

Davis/Hicks Drill Bits

P.O. Box 7906

Midland, TX 79703

Mike Daylong

Magnum Tools, Inc.

230 Bushnell, Suite $\# 3$

San Antonio, TX 78212

Mahlon Dennis

Stratabit

600 Kenrick, Suite Al

Houston, TX 77060

Roderic Deyo

Norton Christensen, Inc.

Diamond Technology Center

2532 South 3270 West

Salt Lake City, UT 84119

Larry Diamond

Smith Dyna-Dril1

P.O. Box C-19576

Irvine, CA 92713

Benny G. DiBona

Drilling Research Laboratory

University Research Park

400 Wakara Way

Salt Lake City, UT 84108

John Dismukes

Exxon Research \& Engineering Co.

Route 22 East

Clinton Township

Annandale, NJ 08801

Harold Doiron

Reed Tool Co.

7000 Hollister, Suite 200

Houston, TX 77040 
Jim Duppstadt

Dresser Mining Services

\& Equipment Div.

P.O. Box 24647

Dallas, TX 75224

Edward Efsic

Stratabit

600 Kenrick, Suite Al

Houston, TX 77060

Ian Faulkner

CRA Limited

55 Collins St.

Melbourne, Australia 3000

Rubin Feenstra

Shell Research B.V.

Volmerlaan 6

2288 GD Rijswijk ZH

The Netherlands

John E. Fontenot

NL Petreleum Services

P.O. Box 60087

Houston, TX 77205

Scott Forrest

Smith Tool

P.O. Box C-19511

Irvine, CA 92713

James Friant

The Robbins Co.

Box 97027

Kent, WA 98031

Dr. Melvin Friedman

Center for Technophysics and Dept. of Geology

College Station, TX 77843

Daniel Garcia

Tulsa University Drilling

Research Projects

Petroleum Engineering Dept.

North Campus Drill Bldg.

2450 Marshall

Tulsa, OK 74110

Michel Geradin

Institut de Mecanique

21 Rue E. Solvay

4000 Liege, Belgium
Malcolm Goodman

EnerTech Engineering \&

Research Co.

2600 Southwest Freeway

Suite 300

Houston, TX 77098

Beverly Geller

Infoplosion

512 Sunderland Rd.

Teaneck, NJ 07666

George Ghusn

BDM Corp.

16300 Christensen Rd.

Suite 315

Seattle, WA 98188

Louis Hibbs, $\mathrm{Jr}$.

General Electric Co.

P.0. Box 8, Bldg. K-1

Schenectady, NY 12301

Gerold Hebert

Dresser Security

P.0. Box 667

Scott, LA 70583

Washan Ho

NL Industries

3000 North Belt

Houston, TX 77032

Larry Hoberock

Amoco Production Company

Research Center

P.O. Box 591

Tulsa, OK 74102

Dr. Michael Hood

University of California

Dept. of Materials Science \&

Minerals Engineering

Berkeley, CA 94720

Stuart Hoenig

Dept. of Electrical Engineering

University of Arizona

Tuscon, AZ 85721

Jessie Holster

Exxon Production Research

P.O. Box 2189

Houston, TX 77001 
Hsin Huang

Norton Christensen, Inc.

Diamond Technology Center

2532 South 3270 West

Salt Lake City, UT 84119

Dr. Virgil E. Johnson, Jr.

Tracor Hydronautics, Inc.

7210 Pindel1 School Rd.

Laure I, MD 20810

Bernard Kear

Exxon Research and Engineering Co.

Clinton Township

Route 22 East

Annandale, NJ 08801

Jim Kingsolver

Smith Tool

P.O. Box C-19511

Irvine, CA 92713

Jack Kolle'

FlowDril Corporation

21414 - 68th Ave. So.

Kent, WA 98032

George A. Kolstad

U.S. Dept. of Energy

Office of Basic Energy Sciences

GPN Bldg., G-226

Washington, DC 20585

Doug Kridler

Valdiamont

3935 Research Park

Ann Arbor, MI 48104

Ergun Kuru

Petroleum Engineering Dept.

Lousiana State University

Baton Rouge, LA 70830

James Langford

Security Division

Dresser Industries

P.O. Box 2467

Dallas, TX 75224

B. J. Livesay

Livesay Consultants

2616 Angel1 Ave.

San Diego, CA 92122
Harvey E. Mallory

P.O. Box 54696

Tulsa, OK 74155

John Marsh

BDM Corp.

1801 Randolph Rd SE

Albuquerque, NM 87106

Ed Martin

Mobil Research \&

Development Corporation

Dallas Research Laboratory

13777 Midway Rd.

Dallas, TX 75224

Steve Mathis

Exxon Production Research

P.O. Box 2189

Houston, TX 77001

Larry Matson

Stratabit

600 Kenrick, Suite Al

Houston, TX 77060

William Maurer

Maurer Engineering, Inc.

10301 NW Freeway

Suite 202

Houston, TX 77018

Alan McFall

NL Petreleum Services

P.O. Box 60087

Houston, TX 77205

Frank McCaffery

Chevron Oil Research

P.0. Box 446

La Habra, CA 90631

John Melaugh

Melaugh Associates, Ltd.

2651 E. 2lst St.

Tulsa, OK 74114

Keith Millheim

Amoco Production Company

Research Center

P.0. Box 591

Tulsa, OK 74102 
John Minge'

Sohio

4440 Warrensville Ctr. Rd.

Cleveland, $\mathrm{OH} 44128$

Stuart Moffitt

Reed Tool Co.

7000 Hollister, Suite 200

Houston, TX 77040

Roland Mottershead

CRA Limited

55 Collins st.

Melbourne, Australia 3000

Joe Neudecker

Los Alamos National Laboratory

Mail Stop 777

Los Alamos, NM 87545

Robert Nicholson

We11 Production Testing, Inc.

P.0. Box 69

Carlsbad, CA 92008

Paul Pastusek

Reed Tool Co.

7000 Hollister, Suite 200

Houston, TX 77040

Gene Polk

P. O. Box 280

Sandia Park, NM 87047

Lew Pratsch

U.S. Dept. of Energy

Geothermal Technologies Division

Forrestal B1dg., CE-324

1000 Independence Ave., SW

Washington, DC 20585

\section{Steve Pye}

Union Geothermal Division

Union Oil Co. of California

Union Oil Center

461 S. Boylston

Los Angeles, CA 90017

Robert Radtke

Tracor, Inc.

Deerbrook Plaza

9810 FM 1960 By-pass

Suite $270-\mathrm{K}$

Humble, TX 77338
Marshal1 Reed

U.S. Dept. of Energy

Geothermal Technologies Division

Forrestal Bldg., CE-324

1000 Independence Ave., SW

Washington, DC 20585

Troy Reed

Conoco Production Research

P.0. Box 1267

Ponca City, OK 74603

Jim Reichman

FlowDril Corporation

21414 - 68th Ave. So.

Kent, WA 98032

Roger Rinaldi

Resource Technology, Inc.

4555 South Harvard

Tulsa, OK 74135

David Rowley

Drilling Research Laboratory

University Research Park

400 Wakara Way

Salt Lake City, UT 84108

John C. Rowley

Los Alamos National Laboratory

Mail Stop 462

Los Alamos, NM 87545

Bill Schwinkendorf

BDM Corp.

1801 Randolph Rd SE

Albuquerque, NM 87106

Adel Sheshtawy

Tri-Max Corp.

P.O. Drawer 1285

Norman, OK 73070

Bill Short, Jr.

Short Cuts, Inc.

9714 Lanward

Dallas, TX 75238

Alan Sinor

Amoco Production Company

Research Center

P.O. Box 591

Tulsa, OK 74102 
David Sitler

GE Specialty Materials

P.0. Box 568

Worthington, $\mathrm{OH} 43085$

Dwight Smith

Halliburton

Drawer 1431

Duncan, OK 73533

David Sommers

University of Missouri-Rolla

Rock Mechanics Facility

Rolla, MO 65401

Steve Southland

Stratabit

600 Kenrick, Suite A1

Houston, TX 77060

Al Sutko

Conoco Production Research

P.O. Box 1267

Ponca City, OK 74603

Malcolm Taylor

NL Hycalog

15112 Morales Rd.

P.O. Box 60747

Houston, TX 77205

George P. Tennyson

DOE/ALO

P.O. Box 5400

Albuquerque, NM 87115

Mike Thigpen

Stratabit

600 Kenrick, Suite A1

Houston, TX 77060

Martin Thompson

Schlumberger Cambridge Research

P.O. Box 153

Cambridge CB2 3BE

England

Tom Turner

Phillips Petroleum Company

Geothermal Operations

655 East 4500 South

Salt Lake City, UT 84107
D. B. Uthus

U.S. Dept. of Energy

Div. of Oi1, Gas, and Shale Technology

$\mathrm{FE}-33$

Washington, DC 20545

Bruce Walker

Drilling Research Laboratory

University Research Park

400 Wakara Way

Salt Lake City, UT 84108

Tom Warren

Amoco Production Company

Research Center

P.O. Box 591

Tulsa, OK 74102

Dennis Williams

Specialty Materials Dept.

General Electric Co.

5325 Huntley Rd.

Worthington, OH 43085

1521 C. M. Stone

3141 S. A. Landenberger (5)

3151 W. L. Garner (3)

5267 J. R. Kelsey

6200 V. L. Dugan

6227 A. A. Heckes

6240 R. K. Traeger

6241 J. C. Dunn (15)

$6241 \mathrm{~J}$. F. Finger

6242 A. Ortega

6300 R. W. Lynch

6310 T. O. Hunter

6314 J. R. Tillerson

6314 D. A. Glowka (30)

6314 T. E. Hinkebein

7864 D. L. Goodwin

8024 P. W. Dean

9242 L. M. Ford 